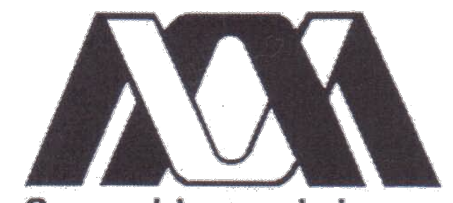

Casa abierta al tiempo
Universidad Autónoma Metropolitana

Unidad Iztapalapa

División de Ciencias Básicas e Ingeniería

Posgrado en Física

\title{
MOdELOS TIPO SOLITÓN PARA \\ HALOS DE MATERIA OSCURA
}

Tesis que presenta

David Castañeda Valle

como requisito parcial para optar por el grado de

Doctor en Ciencias (Física)

Asesor

Dr. Eckehard Erwin Willi Mielke

Jurado calificador

Presidente: Dra. Nora Bretón Báez

Departamento de Física, CINVESTAV

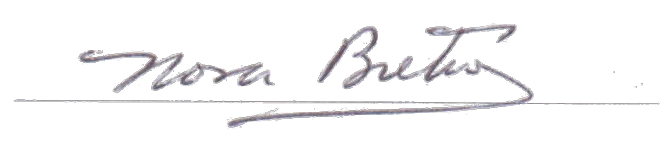

Secretario: Dr. Román Linares Romero

Departamento de Física, UAM - Iztapalapa

Vocal: Dr. Vladimir Manko Semionovich

Departamento de Física, CINVESTAV

Vocal: Dr. Luis Octavio Pimentel Rico

Departamento de Física, UAM - Iztapalapa

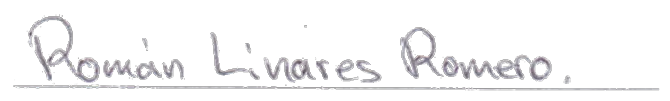

Vocal: Dr. Eckehard E. W. Mielke

Departamento de Física, UAM - Iztapalapa

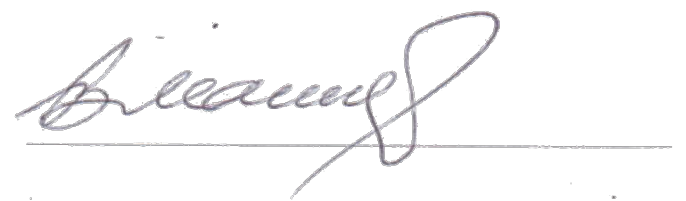

CANCELADO

México, Noviembre de 2015. 

A mis padres, Margarita y Alfredo, a todos mis hermanos y a ti, Eugenia. 



\section{Agradecimientos}

Lo que no experimentas positivamente, lo experimentas negativamente.

Joseph Campbell

Quiero brindar mi más sincera gratitud a todos aquellos que directa e indirectamente participaron en mi vida y durante el desarrollo de este trabajo. La lista sería muy larga: cientos de alumnas y alumnos, decenas de compañeros, algunos amigos y una persona especial. Gracias a todos.

En particular:

- Quiero agradecer al Dr. Eckehard Mielke, por su tiempo y paciencia, que a pesar mis ausencias continuó enviándome bibliografía complementaria, compartiendo fruta y parte de los tantos conocimientos que tiene. Gracias.

- Quiero agradecer a mi familia. A mi mamá, Margarita, que físicamente ya no pudo acompañarme pero en pensamientos sigue conmigo. A mi papá, Alfredo, que a pesar de las pocas conversaciones nunca desistió en su apoyo. A todos mis hermanos, Alfredo, Horacio, Diana, Jesús y Marlen, por sus miles de ayudas sin esperar nada a cambio. A todos ellos, gracias, por haberme tenido paciencia incondicional, y que sin importar las diferencias han permitido que continuemos juntos. Gracias.

- Gracias a Eugenia (Eu), que hiciste que valiera la pena este camino y no desistiera; por dejarme crecer junto a ti y por tantas tantas cosas más, sobre todo por el mejor capítulo. Gracias, incuantificables e inmensurables gracias.

- A Elías (Chelías), ya que no todo es rockear, Beatles, whisky y chela, también es física, gente y familia. Gracias, aunque me regañes. 
- A Carlos (Charlie), Ramón (Chevalier), Valdemar (Vale) y Jesús (Chucho), que más allá de la escuela me permitieron compartir experiencias y trabajo, muchas veces acompañado de una copa más que de un libro. Gracias.

- A los Huguitos, Alex (Chung) y Ernesto, (Neto, Avatar, Pelón, Buda, ...), por que la física y la vida no se vuelvan triviales, mil gracias por las larrrrgas platicas de todo. Gracias.

- A los Juanes, Israel (Rivas) y Carlos (Carlitros), por tantos cafés acompañados de chamba y pláticas, ya fuese en fines de semana y a lo largo de ésta. Gracias.

No puedo pasar por alto mi agradecimiento a los apoyos económicos recibidos, ya sea como beca, de CONACYT, la Unión Europea y el SNI; y como trabajo de, Sycon, la UAM, el CCH, la UACM, la UnADM y la UP. Gracias. 


\section{Índice general}

1 Introducción $\quad 1$

2 Ondas y solitones $\quad 5$

2.1. Panorama histórico ........................ 5

2.2. Solitones y ondas solitarias . . . . . . . . . . . . . 8

2.3. Solitones en física . . . . . . . . . . . . . . . 9 9

2.4. Soluciones tipo solitón . . . . . . . . . . . . . . . . . 12

2.5. Ecuaciones de Bogomol'nyi . . . . . . . . . . . . . . . . 17

2.6. Dinámica de solitones . . . . . . . . . . . . . . . . 19

2.7. Transformaciones de Bäcklund . . . . . . . . . . . . . . 21

2.8. Modelo sine-Gordon . . . . . . . . . . . . . . . 28

3 Halos de materia oscura $\quad 43$

3.1. Materia oscura ..................... . . 43

3.2. Propiedades de la materia oscura . . . . . . . . . . . . . . 46

3.3. Halos de materia oscura . . . . . . . . . . . . . . . 47

3.4. Axión: Un candidato de materia oscura . . . . . . . . . . . . . 49

3.5. Comportamiento solitónico de halos esféricos . . . . . . . . . . . 54

4 Klein-Gordon no lineal $\quad 57$

4.1. Ecuación de Klein-Gordon no lineal . . . . . . . . . . . . . . 58

4.2. Modelo $\phi^{6}$ : Materia oscura escalar . . . . . . . . . . . . . 59

4.3. Potencial tipo Lane-Emden . . . . . . . . . . . . . . . . . . . 61

4.4. Soluciones solitónicas en $(1+1) D \ldots \ldots$. . . . . . . . . . 62

5 Solitones de Klein-Gordon en $(2+1) D \quad 75$

5.1. Modelo sine-Gordon $(2+1)$ D: Uniones de Josephson . . . . . . . . . 75 
5.2. Soluciones de Lane-Emden en $(2+1)$ D . . . . . . . . . . . . . 78

6 Conclusiones y expectativas $\quad 85$

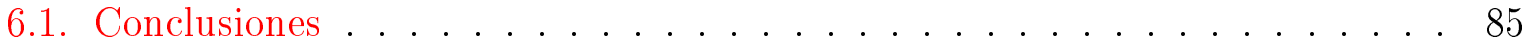

6.2. Expectativas y trabajo futuro . . . . . . . . . . . 87

$\begin{array}{ll}\text { Bibliografía } & 89\end{array}$

$\begin{array}{ll}\text { A Teoría de campos } & 99\end{array}$

A.1. Campo escalar . . . . . . . . . . . . . . . . . . . 99

A.2. Formulación Lagrangiana . . . . . . . . . . . . . . . . . 100

A.3. Simetrías en la naturaleza . . . . . . . . . . . . . . . . 103

A.4. Interpretación de Stueckelberg-Feynman . . . . . . . . . . . . . . 110

B Transformaciones y grupos de Lorentz $\quad 111$

B.1. Transformaciones de Lorentz . . . . . . . . . . . . . . . 111

B.2. Grupos de Lorentz . . . . . . . . . . . . . . . . . 112

C Método de Hirota $\quad 115$

C.1. Transformación . . . . . . . . . . . . . . . 116

C.2. Bilinealización . . . . . . . . . . . . . . . 116

C.3. Expansión perturbacional . . . . . . . . . . . . 117 


\section{Resumen}

El presente trabajo estudia colisiones de solitones de forma semi-analítica. Para esto, en un escenario astrofísico consideramos que posiblemente cierto tipo de materia oscura puede presentar un comportamiento solitónico. Este comportamiento podría presentarse durante colisiones, como las que se suscitan no sólo a nivel de galaxias, también a nivel de cúmulos galácticos. A nivel de cúmulos galácticos se pueden asemejar a lo que se observa en el cúmulo Bullet (1E 0657-56), donde aparentemente los halo de materia oscura correspondientes atraviesan la colisión como si fueran balas sin cambio aparente, mientras que las estrellas y principalmente el gas estelar interacciona y permanece en el centro de la colisión. Esto, asemejando el comportamiento de los solitones, que cuando colisionan pueden interferirse durante el proceso de colisión pero que al dejar ésta recuperan su forma y velocidad. Dicho comportamiento es contradictorio a lo que se observa en otros cúmulos, como el Abell 520.

Los halos de materia oscura bajo ciertas condiciones se considera pueden llegar a formar un condensado de Bose-Einstein. Las partículas que consideramos llegan a formar el condensado son las llamada axiones, partículas escalares aún no detectadas, pero que se consideran uno de los candidatos más prometedores a ser parte de la materia oscura. El condensado formado se considera utilizando un modelo de juguete basado en la ecuación Klein-Gordon no lineal, para la cual obtenemos soluciones solitónicas múltiples en $1+1$ dimensiones, y consideramos soluciones simples en $2+1$ dimensión. 


\section{Capítulo 1}

\section{Introducción}

... el progreso de la física en gran medida

dependerá de los avances de las matemáticas

no lineales, de métodos para resolver

ecuaciones no lineales... y por lo tanto

podemos aprender mediante la comparación

de diferentes problemas no lineales.

Werner Heisenberg

El objetivo planteado, es mostrar que posiblemente cierto tipo de materia oscura puede presentar un comportamiento solitónico durante las colisiones. Colisiones que pueden suscitarse no sólo a nivel de galaxias, sino también a nivel de cúmulos galácticos. A nivel de cúmulos se pueden asemejar a lo que se observa en el cúmulo Bullet (1E 0657-56) o en el MACS J0025.4-1222; vid. Bradač et al. (2008). En estos, aparentemente los núcleos de materia oscura atraviesan la colisión como si fueran balas sin cambio aparente, mientras que las estrellas y principalmente el gas estelar interacciona y permanece en el centro de la colisión; esto asemeja el comportamiento de los solitones que cuando colisionan pueden interferirse en el proceso de colisión pero que al dejarla recuperan su forma y velocidad. Otra semejanza importante al respecto, es cuando dos solitones interaccionan y se anulan durante la colisión, este hecho podría ayudar a explicar el comportamiento de tren descarrilado (Train Wreck) que se menciona para el Cúmulo Abell 520; comportamiento que a la vez, podría dar una explicación a la formación de las llamada galaxias oscuras (Dark Galaxies), de las que VIRGOHI21 se considera una.

El núcleo de materia oscura al que adjudicamos el comportamiento solitónico es una representación del llamado halo de materia oscura o halo galáctico. Este halo, se considera como una extensión en el espacio llena de materia oscura que engloba las estrellas o galaxias, dando lugar a las galaxias y cúmulos, respectivamente. Esta región, es necesaria para justificar el comportamiento observado por el movimiento de las estrellas, en parti- 

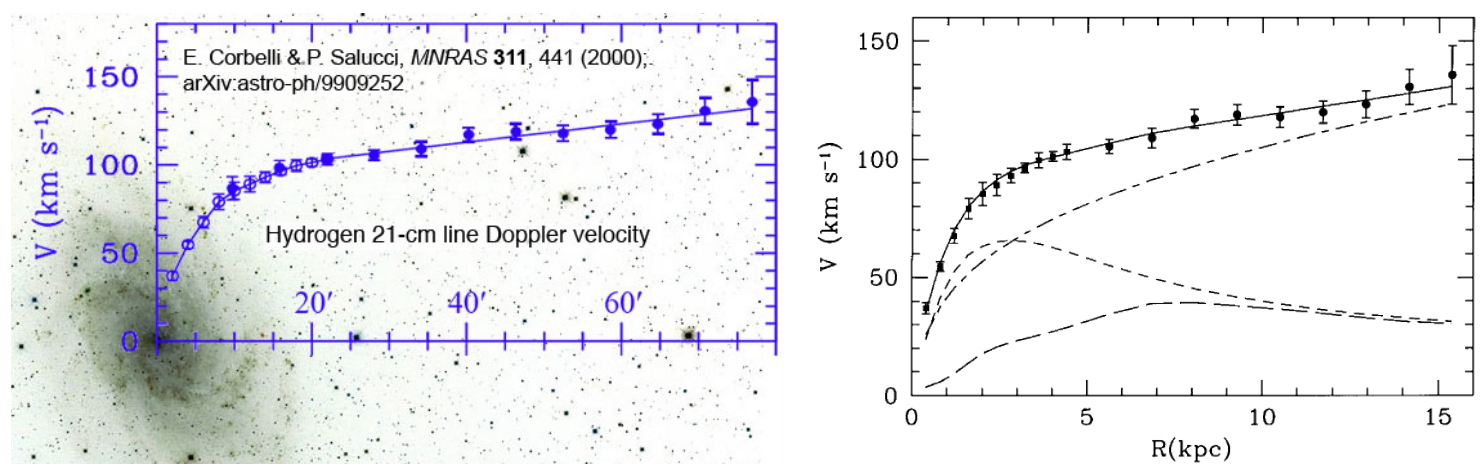

Figura 1.0.1: Las curvas de rotación para la galaxia M33, donde los puntos son datos de velocidad y la línea continua es el mejor ajuste por Corbelli y Salucci (2000), (a) montaje por A. F. Mayer (2011), (b) muestra las contribuciones del halo (---), de disco estelar (--) y contribución del gas (--), así como los dados observados y el mejor ajuste (- - .

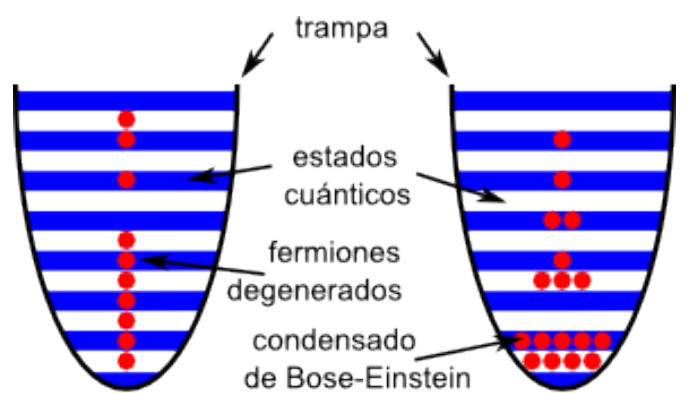

fermiones bosones

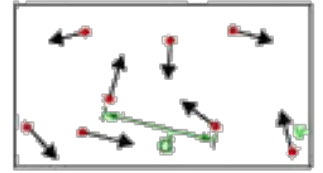

A

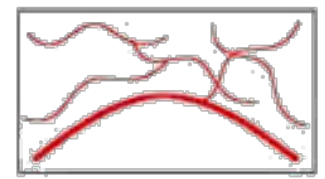

C

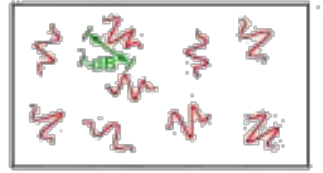

B

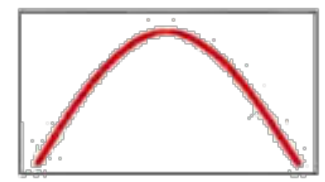

D

Figura 1.0.2: Los bosones como el axión, a diferencia de los feminones, pueden ocupar todos el mismo estado de energía; al tiempo las longitudes de ondas de De Broglie de las partículas se traslapan formando un condensado de Bose-Einstein que puede representarse por una sola función de onda.

cular la velocidad que presentan y cuya representación se conoce como curvas de rotación; Figura 1.0.1. La materia oscura que llenaría este halo, se ha especulado puede estar formado por axiones, que debido a las bajas temperaturas y la trampa gravitacional en la que se encuentran puedan comportarse como un "átomo gigante" llamado condensado de Bose-Einstein, que puede representarse por una única onda localizada.

Por su parte, los axiones son hasta ahora uno de los candidatos más prometedores a formar un tipo de materia oscura. Aún no han sido detectados, y hay en la actualidad experimentos importantes dirigidos a este propósito. La propuesta de esta parícula escalar surgió como una solución a un problema de la teoría de partículas, la invariancia de $C P$. Partículas que a bajas temperaturas puede acomodarse a un nivel de energía bajo dando origen a un condensado de Bose-Einstein; vid. Figura 1.0.2.

En aras de justificar y explicar un acercamiento a una posible solución, el trabajo 
presenta primero un panorama sobre los distintos aspectos involucrados: solitones, materia oscura y en particular del axión. Posteriormente combinando los conceptos planteamos soluciones tipo solitón para halos de materia oscura cuyo comportamiento lo estudiamos vía una ecuación de Klein-Gordon no lineal.

Siendo más específicos la presente exposición esta organizada como sigue. Primero en el capítulo ONDAS Y SOLITONES hacemos una introducción de lo que son las ondas solitarias y los solitones, si bien, la exposición es breve y hasta cierto punto parcial, el objetivo de ésta es presentar de una manera conocida parte de los conceptos que aplicamos durante el trabajo. Es por esto, que más que presentar una lista casi interminable de términos, métodos y ecuaciones en situaciones particulares, presentamos un ejemplo bastante general y conocido. Mencionamos brevemente como obtener soluciones estáticas, dinámicas y su equivalencia, a la vez revisamos la mayor distinción que consideraremos entre los solitones con y sin carga topológica. En el capítulo Modelo SINE-GORDON desarrollamos algunos conceptos utilizados a lo largo del capitulo mencionado, al mismo tiempo, algunos conceptos aplicados. A la vez que cuando analicemos el modelo de sine-Gordon no aplicaremos todos los conceptos expuestos y similarmente no reproducimos todo lo que hacemos en esta sección para los solitones de Lane-Emden.

En el capítulo Halos DE MATERIA OsCuRA presentamos uno de los propósitos del trabajo, describimos muy brevemente lo que implica el modelo de materia oscura escalar, pero principalmente uno de los posibles candidatos, el axión. Justificando cómo podemos aplicar el modelo de sine-Gordon para este campo pseudoescalar, mostramos (sin detalles) que utilizando un modelo diferente pero de características similares aplicado a un halo de materia oscura se logra cualitativamente tener un comportamiento solitónico. En este aspecto es importante recalcar que varios fenómenos físicos permiten una descripción en funciones tipo solitón (ser localizados y con decaimiento exponencial lento) aunque sólo se estudie el solitón simple. La extensión de lo anterior se da en el capítulo KLEIN-GORDON NO LINEAL al intentar encontrar una solución doble-solitón bajo las condiciones de un halo de materia oscura. Para esto, utilizando un modelo de juguete de Lane-Emden pero aproximado a sine-Gordon, usamos mapeos propios de un modelo en el otro. De manera general esto busca pistas sobre el comportamiento de la materia oscura durante colisiones. Parte de estos resultados, han dado lugar a dos publicaciones en revistas de alto prestigio, vid. Castañeda Valle y Mielke (2013, 2014).

Buscando extender aún más los resultados sobre el modelo, esta vez respecto a la cantidad de coordenadas espaciales, el capítulo Solitones DE KLEIn-Gordon En $(2+1)$ D intenta mostrar que, si bien, el modelo no es totalmente integrable, sí se pueden obtener más soluciones solitónicas que las encontradas en los capítulos anteriores. Utilizando en parte el modelo de sine-Gordon, justificamos la necesidad de encontrar soluciones, ya sean 
exactas o aproximadas. Es claro que esto es sólo el principio, ya que nos hemos limitado en los métodos implementados. Una breve discusión al respecto es lo que planteamos en el último capítulo CONCLusiones y ExpeCtativas. Éste último plantea además, las conclusiones finales y otras posibles extensiones al desarrollo hecho durante el trabajo.

Adicionalmente presentamos tres apéndices: TEORÍA DE CAMPOS, TrANSFORMACIONeS y Grupos de Lorentz y Método De Hirota. Los dos primeros para presentar un resumen de conceptos y fórmulas, no una exposición exhaustiva. El último expone el Método de Hirota, que es quizá la primera estrategia para continuar el trabajo y explorar otros escenarios. 


\section{Capítulo 2}

\section{Ondas y solitones}

Las ondas son una manera muy práctica y común que se utiliza para representar fenómenos que se propagan (evolucionan) temporalmente. La concepción y conocimiento más comunes de estos objetos es cuando la onda se propaga de manera periódica; sin embargo, esto no se da en la mayoría de casos, sobre todo debido a agentes dispersivos y disipativos, que aparecen en la naturaleza misma y a la "brevedad" de tiempo que podemos observar los fenómenos de estudio.

Otras situaciones son cuando los fenómenos de estudio se pueden localizar espacial o temporalmente, pueden utilizarse ondas solitarias, este tipo particular de ondas se caracterizan por obedecer teorías no lineales.

Matemáticamente existen entes que combinan estas particularidades:

- pulsos localizados en el tiempo y/o espacio,

- ecuaciones de evolución/propagación no lineales, e

- intervención de agentes dispersivos/disipativos,

un tipo particular de estos entes son los llamados solitones.

\subsection{Panorama histórico}

La narración histórica que entre otros cuentan Remoissenet (1996) y Porter et al. (2009); nos habla de que el primer reporte de una onda solitaria fue hecho por el ingeniero John S. Russell. Russell, quien en agosto de 1834 a lo largo del Union Canal, cerca de Escocia, observó una onda de perfil redondeado bien definido, que avanzaba sin cambiar de forma y sin disminuir de velocidad.

Cuenta la historia, que esta observación lo motivó a realizar una serie de experimentos en estanques y canales, mismos que le permitieron cuantificar sus observaciones. Dichas 


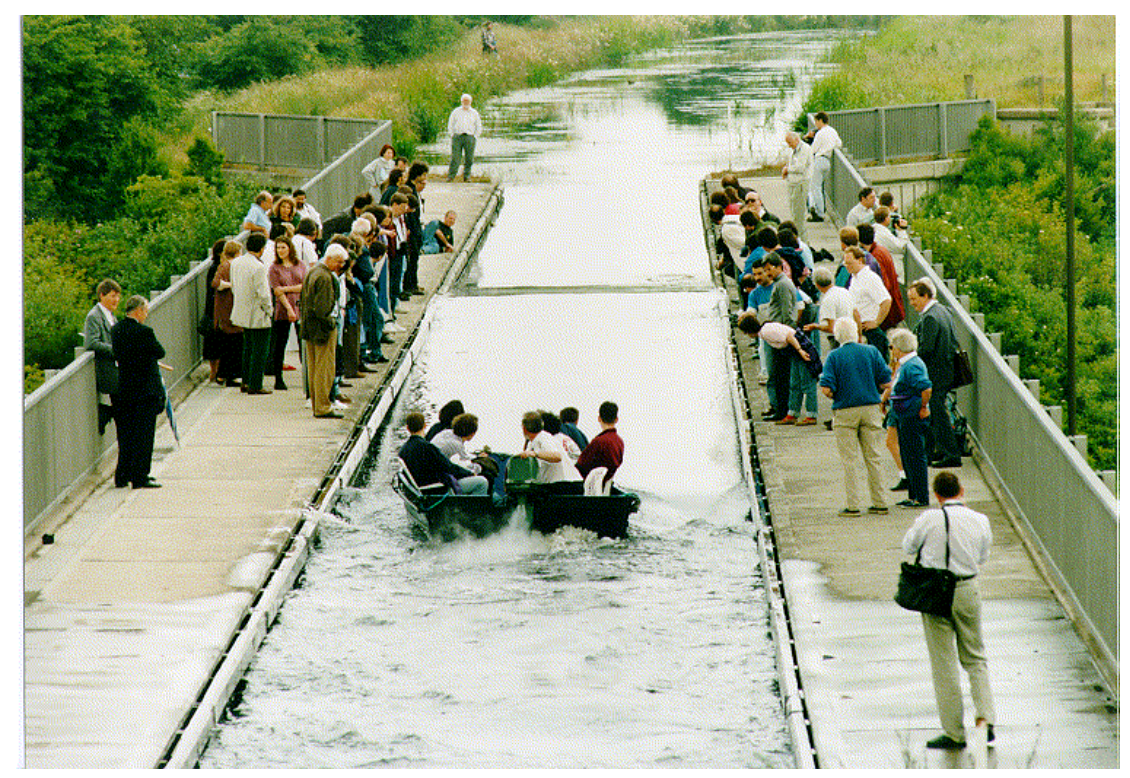

Figura 2.1.1: En 1995, conmemorando los 50 años del problema FPU, se realizó un intento de recrear la onda observa por Russell en el Union Canal.

observaciones Russell las presentaría en una publicación en 1844, en la cual, exponía las siguientes propiedades para ondas solitarias en agua:

I) Un perfil de campana localizado, que viaja con forma y velocidad permanentes.

II) Que en aguas tranquilas, la velocidad de propagación es función de la amplitud $A$, la profundidad del canal $h$ y la gravedad $g$

$$
v=\sqrt{g(h+A)},
$$

como consecuencia de esto, ondas de mayor amplitud se desplazan a mayor velocidad que ondas pequeñas.

III) Que las ondas de este tipo se pueden cruzar una a otra sin presentar cambio alguno de ninguna clase.

IV) Que una elevación inicial de agua, puede evolucionar, ya sea en una sola onda solitaria, una onda solitaria y una onda residual o en dos o más ondas solitarias con o sin onda residual; esto dependiendo de la altura y ancho de la elevación.

v) Que una depresión inicial de agua no produce ondas solitarias; produce en cambio un tren de ondas que aumenta en longitud y decrecen en amplitud.

Estas observaciones contradecían trabajos de George B. Airy (1845) en aguas poco profundas, y de George G. Stokes (1847) referentes a la periodicidad de las ondas. Sin 
embargo, Henry É. Bazin (1865) realizo unos experimentos confirmando las controvertidas observaciones de Russell. Posteriormente, Joseph Boussinesq (1871) y Lord Rayleigh (1876) independientemente, resolviendo la controversia; al mostrar matemáticamente que ignorando la disipación, el incremento en la velocidad local es balanceado con el decrecimiento asociado a la dispersión, conduciendo a un perfil permanente.

Décadas después en 1895, dos físicos holandeses, Diederick Korteweg y su alumno Gustav de Vries, dedujeron y resolvieron en el caso más simple una ecuación en derivadas parciales no lineales de la forma

$$
u_{t}+\alpha u u_{x}+\beta u_{x x x}=0
$$

una ecuación conocida hoy en día como ecuación Korteweg-de Vries o simplemente KdV. Esta ecuación expresa que la tasa de cambio en el tiempo de la altura, está gobernada por dos términos: uno no lineal, responsable de que las velocidades dependan de la amplitud; y uno lineal, responsable de que la dispersión dependa de la longitudes de onda. Korteweg y de Vries mostraron que pueden encontrarse soluciones periódicas que llamaron ondas cnoidales, además, encontraron una solución localizada que reproducía una onda solitaria, tal como la descrita por Russell.

A principios de 1960, en la Universidad de Princeton, Norman Zabusky y posteriormente Martin Kruskal, a partir de simulaciones numéricas del problema FPU ${ }^{1}$; simulaciones realizadas en colaboración con Gary Deen de los Laboratorios Telefónicos Bell. Encontraron que algunas ondas tipo pulso pueden propagarse en un sistema modelado por la ecuación KdV. Las simulaciones, además mostraron que estas ondas podían pasar una a través de la otra y preservar su forma y velocidad después de una colisión. Fue así, que acuñaron el término solitón para aquellas ondas solitarias. Término que remarca, el hecho que son ondas con propiedades de partículas mejor llamadas cuasipartículas.

La ecuación KdV no es la única que presenta este tipo de soluciones. Otro caso de gran importancia tecnológica se dio en 1971, cuando los investigadores Vladimir E. Zakharov y Aleksei B. Shabat de la URSS, descubrieron una versión no lineal de la ecuación de Schrödinger o conocida también como NLS. Ésta presenta soluciones de comportamiento similar, las cuales se utilizan en fibras ópticas para aplicaciones tan trascendentales como la transmisión en los cables interoceánicos modernos TAT, así como en otras aplicaciones de telecomunicaciones.

\footnotetext{
${ }^{1}$ E. Fermi, J. Pasta y S. Ulam estudiaron numéricamente en 1955, en el laboratorio de Los Álamos, cadenas formadas por masas unidas por resortes no lineales. Estos sistemas reciben el nombre de cadenas FPU o simplemente problema FPU. Estos sistemas presentan la peculiaridad que cuando las desviaciones de las posiciones de equilibrio son muy pequeñas, el sistema de ecuaciones puede desacoplarse y la dinámica puede ser descrita por la superposición de modos normales, representados por ondas sinusoidales mutuamente independientes.
} 


\subsection{Solitones y ondas solitarias}

El planteamiento de ondas junto con las características de partículas permiten un análisis más general, la teoría de campo. Desde esta teoría se puede establecer un criterio para definir un solitón, ya sea sobre la densidad de energía $\rho(t, x)$ o sobre los campos $\phi_{i}(t, x)$. Rajaraman (1982) establece la densidad de energía, ya que es común en física de partículas y en sistemas donde la energía se conserva; por otra parte Scott et al. (1973), lo establece en el campo lo que da cabida a sistemas que no conserven la energía, además de ser una manera más simple.

En el caso de ondas relativistas, el criterio sobre la densidad de energía $\rho(x, t)$, requiere una energía localizada y finita. Por lo cual ésta puede minimizarse

$$
E[\phi]=\int d x \rho(t, x)=\text { cte }
$$

y puede además, establecerse la energía mínima en $E=0$, para esto se pide que

$$
\rho(t, x) \rightarrow \rho\left(\frac{\gamma}{c}(c t-\beta x), \gamma(x-c \beta t)\right)
$$

es decir, una invariancia ante transformaciones de Lorentz. Esto implica que la densidad de energía debe moverse sin distorsionarse a una velocidad constante; esta definición permite encontrar una mayor cantidad de soluciones, mientras que plantear el criterio sobre los campos

$$
\phi(t, x) \rightarrow \phi\left(\frac{\gamma}{c}(c t-\beta x), \gamma(x-c \beta t)\right),
$$

puede excluir ondas solitarias cargadas. Coleman (1985) restringe menos su definición, pidiendo únicamente que la solución no sea singular y que la densidad de energía esté localizada, se cumpla o no la ecuación (2.2.2). Ejemplos muy importantes en ambos escenarios son bien conocidos; mientras que el modelo de sine-Gordon no cumple la condición en la densidad (2.2.2); a la vez que el modelo de $\phi^{4}$ la satisface; ver Sección 2.8.

Cada una de estas definiciones no son universalmente aceptadas; sin embargo, coinciden en dos propiedades claras. Éstas sirven para establecer una definición de onda solitaria y solitón:

P1 Un perfil localizado.

P2 Tras una colisión, las ondas recuperan su forma y velocidad.

Puntualicemos que el significado de las propiedades mencionadas para el fenómeno que queremos estudiar. La propiedad P1 nos habla de dónde está el fenómeno, se refiere a que, en un tiempo dado, ya sea el campo o la densidad de energía, se extienda en una región finita del espacio. Mientras que la propiedad P2 tiene que ver con la vida del fenómeno, se 
refiere a que, dos fenómenos de naturaleza similar, cada uno independiente en el tiempo y espacio se encuentran, interactuaran y posteriormente se alejan, sólo quedando como huella del encuentro un posible retraso espacial-temporal; y cada uno retoma su forma y velocidad, tal como antes de la interacción.

De esto podemos decir que, se habla de una onda solitaria cuando el fenómeno sólo cumple la primera propiedad (P1). Y cuando satisface ambas propiedades (P1 y P2) para tiempos muy anteriores y muy posteriores al encuentro, a esa onda solitaria se le llama solitón. En un contexto histórico estos conceptos se pueden establecer como sigue:

Onda solitaria, como fue descubierta experimentalmente por Russell en 1834, es una onda localizada que se propaga a lo largo de una sola dirección espacial, sin cambiar de forma $(\mathrm{P} 1)$.

Solitón, como fue descubierto numéricamente por Zabusky y Kruskal a finales de los 1960's, es un pulso coherente de gran amplitud localizado muy estable (P1) que matemáticamente es la solución exacta de una ecuación de onda cuyo perfil y velocidad no son alterados por una colisión con otra onda similar (P2).

\subsection{Solitones en física}

El último concepto de solitón planteado es muy específico e ideal en el contexto matemático, este último implica que el modelo del sistema sea integrable, al menos parcialmente. En el mundo de la física, sin embargo; se trata con sistemas reales. En los que es difícil concebir todos los agentes que intervienen en un fenómeno; por lo cual, los modelos deben considerar propiedades aproximadas. Con esto, la versión física se presenta en sistemas donde efectos no solitónicos o perturbacionales, tales como mecanismos de perdida de energía por fricción, fuerzas externas de control, u otros no controlables del todo, son inevitables; por lo que las ondas solitarias o los solitones, aún de larga vida, son sólo meta-estables. Además, la ausencia de información para todo tiempo impide probar el cumplimiento asintótico de las propiedades de onda solitaria o solitón.

Es común encontrar en la literatura que tanto a ondas solitarias y solitones se les llame simplemente solitones cumplan o no la propiedad P2. Sin embargo, los solitones deben satisfacer las ecuaciones dinámicas de interacción, por lo cual, todos los solitones son ondas solitarias, pero no todas las ondas solitarias son solitones. También se debe notar que las soluciones estáticas localizadas no singulares son ondas solitarias; para que éstas sean solitones deben satisfacer ambas propiedades, considerando la dependencia temporal. En algunos casos, esto último únicamente requiere aplicar una transformación de invariancia del sistema (v.g. transformaciones de Lorentz o Galileo) a la solución estática. 
Sin embargo, en otros sistemas debe resolverse directamente el sistema con dependencia temporal.

Cualitativamente la onda solitaria o solitón puede entenderse como la representación del balance entre efectos de dispersión y efectos no lineales. Aunque los solitones también pueden encontrarse como resultado de un balance dinámico entre disipación y no linealidad. Es así que tenemos tres fenómenos importantes para la formación de solitones: disipación, dispersión y no linealidad.

Disipación Los fenómenos de disipación se refieren a cuando la amplitud de onda decrece con el paso del tiempo. La manera más simple de determinar la disipación, es estudiando la relación de dispersión $\omega(k)$. En el caso de una onda oscilante, separando la parte real e imaginaria se puede escribir

$$
\omega(k)=\omega_{R}+i \omega_{I} .
$$

Cuando la parte imaginaria $\omega_{I}>0$, la onda se vuelve inestable; ya que crece sin control mientras que de ser $\omega_{I}<0$ la onda decae cuando avanza el tiempo.

Dispersión Los fenómenos dispersivos se presentan cuando la velocidad de grupo y la velocidad de fase dependen del número de onda. Por tal motivo, ondas con una relación de dispersión lineal $\omega(k)$ y con una velocidad de propagación constante son llamadas no dispersivas. Si la relación es no lineal, se les llaman ondas dispersivas. Esto se manifiesta cuando los componentes de una onda se propagan a diferentes velocidades, estos se separan o se dispersan unos respecto de otros.

No linealidad Los efectos no lineales se presentan cuando una interacción sobre un sistema genera una salida que no es proporcional a la entrada, esto es muy común en la naturaleza y ciencias sociales. En sistemas de ondas la manifestación más común es cuando la frecuencia de oscilación depende de la amplitud, es decir, se viola el principio de superposición lineal.

\subsubsection{Solitón de KdV}

Quizá el solitón más famoso es el KdV, que como hemos mencionado tiene una versión tangible, la que fue observada por Russell y es el primer reporte del fenómeno. Posteriormente, éste fue observado por Enrico Fermi, John Pasta y Stanislaw Ulam en el problema que hoy lleva sus iniciales, el llamado problema FPU. Este problema es reconocido por muchos como el nacimiento de la matemática experimental; Weissert (1997); Porter et al. (2009). 

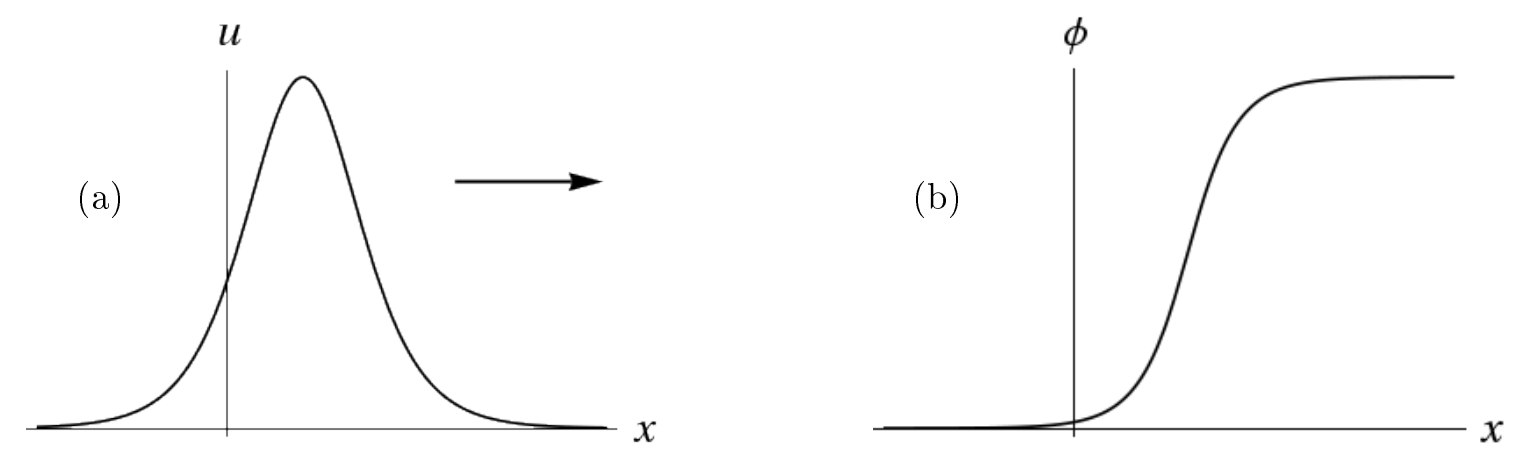

Figura 2.3.1: Representación más común de los solitones de (a) Korteweg-de-Vries, tipo lump y (b) sine-Gordon, tipo kink.

La famosa ecuación tiene la forma

$$
u_{t}+\alpha u u_{x}+\beta u_{x x x}=0
$$

donde $u=u(t, x)$ y los coeficientes $\alpha$ y $\beta$ se determinan de las propiedades del medio y pueden ser funciones de las coordenadas o constantes, en este último caso una solución exacta es

$$
u(t, x)=\frac{3 p^{2}}{\alpha} \operatorname{sech}^{2}\left[\frac{1}{2 \sqrt{\beta}}\left(p x-p^{3} t+\phi_{0}\right)\right],
$$

donde $\phi_{0}$ es la localización del extremo en $t=0$ y $p \in \mathbb{R}$ genera una familia monoparamétrica de soluciones; Figura 2.3.1a.

\subsubsection{Solitón de sine-Gordon}

El modelo de sine-Gordon del cual hablaremos posteriormente, casi tan famoso como el $\mathrm{KdV}$, se presenta en muchos fenómenos en distintas áreas de conocimiento. Sistemas que van desde líneas de trasmisión a flujos magnéticos (fluxones); de estructuras celulares vivas a materia oscura (axiones); vid. Dodd et al. (1982); Ivancevic y Ivancevic (2013).

$\mathrm{Su}$ ecuación correspondiente es

$$
\frac{1}{c^{2}} \phi_{t t}-\phi_{x x}+\sin \phi=0
$$

donde $\phi=\phi(t, x)$ y una solución exacta es

$$
\phi(t, x)=4 \arctan \left[\exp \left( \pm \gamma\left(x-x_{0}-v t\right)\right)\right]
$$

para la cual $v$ es la velocidad de la onda, $\gamma$ el factor de Lorentz y $x_{0}$ la posición inicial del solitón. Este modelo veremos que puede entenderse como un caso particular de un modelo más general conocido como Klein-Gordon no lineal, que trataremos más adelante; Figura 2.3.1b. 


\subsection{Soluciones tipo solitón}

Encontrar soluciones solitónicas no siempre es una tarea simple y sistematizada; de hecho hallar estas soluciones es sólo una parte del problema, otra, las características de la misma. Estas soluciones pueden obtenerse por distintas técnicas y métodos como pueden ser: las transformaciones de Bäcklund, los método de dispersión inversa, directo de Hirota, de Zakharov-Shabat, etc. En general, cada método está dirigido para buscar y explotar o aprovechar alguna característica propia del sistema en cuestión. La monografía de Infeld y Rowlands (1990) presenta un panorama de estos métodos y transformaciones.

Es importante notar que la mayor parte de las soluciones son no perturbativas, esto es, no se pueden obtener partiendo de una solución de la parte lineal correspondiente, salvo que la perturbación sea no lineal; Kivshar y Malomed (1989).

Para mostrar algunas características consideraremos la ecuación de onda no homogénea; es decir, una ecuación de la forma

$$
\frac{1}{c^{2}} \frac{\partial^{2}}{\partial t^{2}} \phi-\nabla^{2} \phi=-F(\phi)
$$

convenientemente se puede considerar el caso en el que

$$
F(\phi)=\frac{\partial}{\partial \phi} V(\phi)
$$

es decir, $F(\phi)$ proviene de un potencial; el caso cuando $F(\phi)=0$ obtenemos la ecuación de onda libre, que es no dispersiva y periódica.

\subsubsection{Soluciones estáticas}

La opción más simple para buscar una solución solitónica, es comenzar con soluciones estáticas $\phi=\phi(x)$. Esto implica que el perfil sea permanente en el tiempo y no se mueva.

De manera práctica, esto requiere cancelar las variaciones temporales, y posteriormente reintroducir el tiempo utilizando las transformaciones de simetría; v.g. transformaciones de Lorentz. Con esto la ecuación (2.4.1) se reduce en $(1+1) \mathrm{D}$ a

$$
\frac{\partial^{2} \phi}{\partial x^{2}}=\frac{\partial V(\phi)}{\partial \phi}
$$

esta ecuación puede integrase para obtener

$$
\frac{d \phi}{d x}= \pm \sqrt{2 V(\phi)}
$$

Lo que nos lleva a la identidad del virial

$$
\int_{-\infty}^{\infty} d x\left[\frac{1}{2}\left(\frac{d \phi}{d x}\right)^{2}\right]=\int_{-\infty}^{\infty} d x V(\phi)
$$


que relaciona los términos de gradiente y potencial en el Lagrangiano.

Además, al considerar la densidad de energía (A.2.23) obtenemos

$$
\rho(x)=\frac{1}{2}\left(\frac{d \phi}{d x}\right)^{2}+V(\phi),
$$

esta expresión muestra dos contribuciones a la energía potencial: una tipo gradiente y otra de la función potencial. Ambas contribuciones deben proporcionar las características del solitón. Para esto, como la energía debe tener un valor finito y estar localizada, se deben cumplir las condiciones de frontera siguientes

$$
\lim _{|x| \rightarrow \infty} \frac{d \phi}{d x} \rightarrow a_{0}, \quad \lim _{|x| \rightarrow \infty} V(\phi) \rightarrow b_{0}
$$

donde $a_{0}$ y $b_{0}$ son constantes que, sin perder generalidad, pueden anularse o absorberse en el potencial $V(\phi)$.

\subsubsection{Soluciones estacionarias}

Otro acercamiento al buscar una solución solitónica puede lograrse al considerar el perfil permanente; es decir, aprovechar una simetría que permita una solución estacionaria.

Consideremos la ecuación (2.4.1) donde con (2.4.2) tenemos un término de potencial; y otro correspondiente al D'Alembertiano aplicado a la función (campo) $\phi=\phi(t, \vec{x})$, correspondiente a un término cinético. Para encontrar una solución con un perfil permanente, tomemos en cuenta la simetría relativista, así como el factor de Lorentz $\gamma^{-1}=\sqrt{1-v^{2} / c^{2}}$. Entonces escribamos la función como $\phi \rightarrow \phi(s)$ donde la fase $s$ y la posición $\vec{x}-\vec{v} t$ referentes a un sistema de coordenadas móvil a velocidad constante $\vec{v}$, hacen que la onda permanezca estacionaria. Usando esto, el operador D’Alembertiano, reducido a sólo 1+1 dimensiones se puede escribir como

$$
\square=\gamma^{2}\left(\frac{v^{2}}{c^{2}}-1\right) \frac{d^{2}}{d s^{2}}=-\frac{d^{2}}{d s^{2}}
$$

De está forma, la ecuación de onda (2.4.1), se puede escribir como

$$
\frac{d^{2} \phi}{d s^{2}}=\frac{\partial V(\phi)}{\partial \phi}
$$

esta expresión es totalmente análoga a (2.4.3), y permite escribir expresiones análogas a las ecuaciones (2.4.4-2.4.7). Esto implica que para la simetría considerada para este tipo de ecuaciones ambos análisis son equivalentes. 


\subsubsection{Soluciones topológicas}

Un tipo más de soluciones o análisis para obtener éstas, es el de las caracterizadas por un indice topológico ${ }^{2}$. Dicho índice es una medición de una característica geométrica propia de algunos sistemas. Esta característica da lugar a organizaciones estructurales de gran interés en distintos campos de la física, en campos tan variados como física de altas energías, materia condensada, óptica, cosmología, líneas de transmisión, biofísica, etc.

Desde este punto de vista, las estructuras se clasifican como topológicas y no topológicas, linealmente estables e inestables, respectivamente. Las estructuras topológicas o también llamadas tipo kink, pueden presentarse en materia condensada como interfaces, en cosmología sirven para explicar la formación de estructuras en el universo temprano, así como para modelos de energía oscura, entre otros. Por su parte, las estructuras no topológicas o tipo lump, pueden presentarse en el transporte de carga en cadenas diatómicas, en la formación de q-balls, branas taquiónicas, así como para explicar propiedades de materia oscura. Dentro de las estructuras no topológicas caen aquellas con la forma de campana; v.g. el solitón de KdV. Además, existen modelos como el sine-Gordon que presentan tanto solitones topológicos como no topológicos.

Dependiendo de las dimensiones involucradas en el problema y el valor del índice topológico los solitones pueden recibir distintos nombres. Así por ejemplo, estructuras tipo solitón son conocidas con nombres como kinks, lumps, vórtices, dromiones, monopolos, instantones, etc.

\section{Carga topológica}

Una consecuencia directa del índice topológico es la existencia de una carga conservada, en este caso llamada carga topológica. Para ésta, consideremos los puntos críticos del potencial para un instante $t_{0}$. Como las soluciones deben poseer una energía finita y tener un comportamiento asintótico definido, además, tomaremos aquellos que sean ceros, para que correspondan a los estados base del sistema. Esto se logra ya que siempre podemos agregar una constante al potencial.

Dicho lo anterior sean $\bar{\phi}_{i}$, los puntos críticos del potencial que además son ceros, esto es

$$
V^{\prime}\left(\bar{\phi}_{i}\right)=0, \quad V\left(\bar{\phi}_{i}\right)=0
$$

estos puntos forman un conjunto discreto no necesariamente finito, en los cuales el funcional de energía es también minimizado, ya que el campo $\phi(t, x)$ es constante en el

\footnotetext{
${ }^{2}$ La generalización del concepto requiere utilizar el llamado grado de Brouwer, para la suavidad del mapeo de las superficies.
} 
espacio-tiempo

$$
E\left[\bar{\phi}_{i}\right]=0
$$

El comportamiento asintótico lo veremos a partir de los puntos críticos. Sea $\phi \rightarrow \bar{\phi}_{i}$ cuando $x \rightarrow-\infty$; a la vez $\phi \rightarrow \bar{\phi}_{j}$ cuando $x \rightarrow+\infty$, ya sea que $\phi_{i}$ y $\phi_{j}$ sean iguales o no, y permiten distinguir dos tipos de estructuras:

I) topológicas o tipo kink (antikink), cuando

$$
\lim _{x_{p} \rightarrow \infty} \phi\left(x_{P}\right) \rightarrow \bar{\phi}_{i}, \quad \lim _{x_{p} \rightarrow-\infty} \phi\left(x_{P}\right) \rightarrow \bar{\phi}_{i+1},
$$

siendo $\phi_{i} \neq \phi_{i+1}, P x=x_{p}, P$ es el operador de paridad y

II) no-topológicas o tipo lump, cuando

$$
\lim _{|x| \rightarrow \infty} \phi(x) \rightarrow \bar{\phi}_{i}
$$

En sistemas con más de un punto critico se puede definir el índice topológico el cual se conserva en el tiempo. Esta cantidad como otras conservadas juegan un papel importante; por ejemplo en teoría cuántica de campos (QFT), donde está relacionada con un número cuántico de estados de partículas. Sin embargo, tiene un origen diferente al de otras cantidades conservadas. En el caso especial de solitones las soluciones no singulares de energía finita, ya sean estáticas o dependientes del tiempo, están determinadas por los mínimos del potencial $V(\phi)$ para cualquier instante $t$ cuando tendemos a cada infinito espacial.

Para las estructuras mencionadas, el conjunto de puntos $\overline{\phi_{i}}$ dividen al espacio de soluciones de energía finita no singulares en regiones llamadas sectores topológicos, caracterizados por dos índices nombrados a partir de valores independientes del tiempo de $\bar{\phi}_{+}$ y $\bar{\phi}_{-}$, donde $\bar{\phi}_{ \pm}=\bar{\phi}(x= \pm \infty)$. La energía finita de la solución de cada sector impide que una modificación continua del campo tenga lugar debido a la solución de otro sector, por lo que los sectores son topológicamente no conectados, es decir, las modificaciones continuas se dan en el mismo sector, por ejemplo la evolución temporal. Cuando $V(\phi)$ tiene un solo mínimo, sólo hay un valor permisible para $\bar{\phi}_{+}=\bar{\phi}_{-}$, por lo que sólo hay dos sectores.

En cada sector se puede definir una cantidad llamada carga topológica, para esto

$$
J^{\mu}:=\frac{1}{\left|l_{i j}\right|} \epsilon^{\mu \nu} \partial_{\nu} \phi,
$$

la densidad de corriente topológica, donde $l_{i j}$ en este caso $(1+1) \mathrm{D}$, es la distancia ${ }^{3}$ entre los puntos $\overline{\phi_{i}}$ y $\overline{\phi_{j}}$ asociados al sector topológico en cuestión, además $\epsilon^{\mu \nu}$ es el tensor de Levi-Civita en $1+1$ dimensiones, con $\epsilon_{01}=+1$.

\footnotetext{
${ }^{3}$ En un caso general de $n$ dimensiones, $l_{i j}$ es el área de la superficie de una esfera unitaria en $n$-dimensiones.
} 
Similarmente al teorema de Noether (Sección A.3.1.1), esta corriente puede agregarse como una divergencia total en forma de un Lagrangiano topológico (geométrico) que es no dinámico; vid. Patani et al. (1976); de la forma

$$
L_{\text {top }}=\partial_{\mu}\left(\frac{1}{\left|l_{i j}\right|} \epsilon^{\mu \nu} \partial_{\nu} \phi(c t, x)\right)=\partial_{\mu} J^{\mu},
$$

ya que de hecho corresponde a una ley de conservación para la carga topológica

$$
\partial_{\mu} J^{\mu}=0
$$

Este último hecho también se corresponde con el lema de Poincaré para funciones con derivadas continuas $\epsilon^{\mu \nu} \partial_{\mu} \partial_{\nu}=0$.

Con lo anterior podemos definir la carga topológica como

$$
Q:=\int_{-\infty}^{\infty} d x J_{0}=\frac{1}{\left|l_{i j}\right|} \epsilon_{01} \int_{-\infty}^{\infty} d x \partial_{x} \phi=\frac{1}{\left|l_{i j}\right|}\left[\bar{\phi}_{+}-\bar{\phi}_{-}\right]
$$

donde la cantidad $Q$ es entero valuada, ya que es la diferencia entre dos índices, $\overline{\phi_{i_{+}}} /\left|\overline{\phi_{i}}\right|$ y $\overline{\phi_{j}} /\left|\overline{\phi_{j}}\right|$. En casos donde el campo $\phi$ es una cantidad física medible, un sector topológico se encuentra determinado no sólo por $Q$, sino también por los valores $\bar{\phi}_{ \pm}$; por otra parte, si la cantidad medible depende sólo de las diferencias de $\phi$, sólo la carga $Q$ es necesaria para determinar el sector.

La diferencia de los dos índices puede tener tres posibles casos: $Q>0, Q<0$ y $Q=0$. En el caso de $Q \neq 0$, tenemos estructuras tipo kink o topológicas como en (2.4.12), en particular si $Q>0$ las llamaremos tipo kink, y si $Q<0$ las llamaremos tipo antikink. El caso de $Q=0$ nos lleva a estructuras tipo lump o no topológicos (2.4.13), que en ciertos casos pueden entenderse como kinks seguidos de antikinks. De tal manera que la carga de un solitón pude entenderse como la superposición de estructuras

$$
Q=(\text { carga de kinks })+(\text { carga de antikinks }) \text {. }
$$

Esta expresión es más general de lo que aparenta, ya que implica la posible presencia de cantidad múltiple de solitones y posiblemente de varios tipos; sin embargo, es una cantidad que debe conservarse.

La topología de un solitón también tiene manifestaciones sobre los sistemas físicos, por ejemplo los solitones no topológicos no modifican el sistema después del su paso; mientras que los solitones topológicos en ocasiones sí modifican la estructura del sistema, dicha modificación puede deberse a un cambio de sector topológico.

Si bien la carga topológica se conserva, su naturaleza es diferente. Mientras que cantidades como energía, momento y carga eléctrica ${ }^{4}$ se conservan por la existencia de simetrías

\footnotetext{
${ }^{4}$ Translaciones temporal y espacial para energía y momento, respectivamente; y transformaciones de norma de $U(1)$ para la carga eléctrica.
} 
continuas del Lagrangiano; la carga topológica se origina en las condiciones de frontera debido a que la energía es finita. Por lo que está relacionada con ciertas violaciones de simetría. Por ejemplo, si el potencial $V(\phi)$ es invariante bajo la transformación de $\phi \leftrightarrow-\phi$, los puntos críticos se pueden ver afectados de la forma $\overline{\phi_{i}} \rightarrow \overline{\phi_{j}} \mathrm{y} \overline{\phi_{j}} \rightarrow \overline{\phi_{i}}$, donde $\overline{\phi_{i}} \neq \overline{\phi_{j}}$, dando origen a lo que en algunas teorías se conoce como rompimiento espontáneo de simetría.

\subsection{Ecuaciones de Bogomol'nyi}

Un punto de partida para estudiar el comportamiento de las ecuaciones estáticas, es comenzar con (2.4.3), así integrando ésta

$$
\int d x \phi^{\prime} \frac{\partial^{2} \phi}{\partial x^{2}}=\int d x \frac{\partial \phi}{\partial x} \frac{\partial V}{\partial \phi}=\int d \phi \frac{\partial V}{\partial \phi}
$$

obtenemos

$$
\frac{1}{2}\left(\phi^{\prime}\right)^{2}=V(\phi)+b
$$

donde $b$ es una constante de integración. Despejando $\phi_{x}$ obtenemos un par de ecuaciones diferenciales, conocidas como ecuaciones de Bogomol'nyi de primer orden; vid. Manton y Sutcliffe (2004),

$$
\frac{\partial \phi}{\partial x}= \pm \sqrt{2 V(\phi)}
$$

Las ecuaciones de Bogomol'nyi nunca involucran derivadas temporales, y sus soluciones son solitones estáticos o configuraciones multisolitón. La constante $b$ se anula debido a las condiciones de frontera (2.4.7). Por definición la función potencial $V(\phi)$ es positiva definida, y ya que la derivada no cambia de signo, la ecuación (2.5.3) nos genera dos funciones monótonas de $x$. Identificaremos tipo kink la solución de pendiente positiva $\phi_{\text {kink }}=\phi_{\mathrm{k}} \mathrm{y}$ tipo antikink la de pendiente negativa $\phi_{\text {antikink }}=\phi_{\overline{\mathrm{k}}}$. Notemos que estas dos soluciones se relacionan a través del operador de conjugación de carga $C$

$$
\phi_{\text {antikink }}=C \phi_{\text {kink }}
$$

La solución no topológica, también puede definirse en términos de estas ecuaciones (2.5.3), aunque en este caso la solución no es monótona y debe ser trabajada por trozos como sigue, para $\phi \leqslant 0$

$$
\frac{\partial \phi}{\partial x}=\left\{\begin{array}{ll}
\sqrt{2 V(\phi)} & \text { con } x>0 \\
-\sqrt{2 V(\phi)} & \text { con } x<0
\end{array},\right.
$$

$\mathrm{y} / \mathrm{o}$ para $\phi \geqslant 0$

$$
\frac{\partial \phi}{\partial x}=\left\{\begin{array}{ll}
-\sqrt{2 V(\phi)} & \text { con } x>0 \\
\sqrt{2 V(\phi)} & \text { con } x<0
\end{array} .\right.
$$


La presencia aquí de dos trozos en (2.5.5) o en (2.5.6) se puede entender con la ayuda de la simetría espacial o de paridad $(x \rightarrow-x)$ del campo $\phi$ en el potencial $V(\phi)$.

Otra manera de escribir las ecuaciones de Bogomol'nyi, es con la ayuda de la llamada función superpotencial $W(\phi)$; Manton y Sutcliffe (2004); Avelar et al. (2007, 2009). Con lo que la relación entre las funciones $V(\phi)$ y $W(\phi)$ es

$$
\begin{gathered}
V(\phi)=\frac{1}{2}\left(\frac{d W}{d \phi}\right)^{2}=\frac{1}{2} W_{\phi}^{2} \\
W(\phi)= \pm \int \sqrt{2 V(\phi)} d \phi
\end{gathered}
$$

resultando que las ecuaciones (2.5.3) para el kink y antikink se pueden escribir simplemente como

$$
\frac{d \phi}{d x}=W_{\phi}, \quad \frac{d \phi}{d x}=-W_{\phi}
$$

La densidad de energía estática (2.4.6) con sus dos contribuciones permite evadir el teorema de Derrick (1964) y admitir soluciones solitónicas estáticas. Para mostrar tal cosa consideremos la desigualdad

$$
\left(\frac{1}{\sqrt{2}} \phi^{\prime} \pm \sqrt{V(\phi)}\right)^{2} \geqslant 0
$$

tras la cual expandiendo la relación, podemos identificar la densidad de energía (2.4.6) y escribir

$$
\frac{1}{2} \phi^{\prime 2}+V(\phi) \geqslant \pm \phi^{\prime} \sqrt{2 V(\phi)}
$$

sustituyendo el superpotencial (2.5.7) e integrando sobre todo el espacio obtenemos

$$
E \geqslant\left|W\left(\phi_{+}\right)-W\left(\phi_{-}\right)\right|
$$

donde $\phi_{ \pm}=\phi(x= \pm \infty)$. Esta expresión nos dice que la energía esta acotada por abajo en términos de datos topológicos, y es llamada cota de Bogomol'nyi. Para satisfacer la igualdad los campos deben ser estáticos $\partial_{t} \phi=0$, y satisfacer las ecuaciones de Bogomol'nyi de primer orden. Utilizando la ecuación (2.5.12), podemos calcular la energía de un solitón tipo kink y tenemos

$$
E_{\mathrm{kink}}=\left|W\left(\phi_{+}\right)-W\left(\phi_{-}\right)\right|
$$

mientas que para uno tipo lump

$$
\begin{aligned}
E_{\text {lump }} & =\left|W\left(\phi_{+}\right)-W(\phi(0))\right|+\left|W(\phi(0))-W\left(\phi_{-}\right)\right| \\
& =2\left|W\left(\phi_{+}\right)-W(\phi(0))\right|
\end{aligned}
$$

Estas expresiones son una manera más simple de obtener la energía que integrar directamente la densidad de energía. 


\subsection{Dinámica de solitones}

En una teoría relativista las ecuaciones de movimiento están determinadas por las ecuaciones de Euler-Lagrange para campos estáticos; y es posible posteriormente hacerlas viajeras a velocidad arbitraria menor que la de la luz $c$ según las transformaciones de Lorentz. Sin embargo, en una teoría no-relativista (v.g. Ginzburg-Landau) no es fácil determinar los campos dependientes del tiempo, por lo que son necesarios datos experimentales; Manton y Sutcliffe (2004).

La analogía de solitones como partículas localizadas es importante para describir su dinámica (movimiento, colisiones, cantidades conservadas, etc.). Esto es cuando los solitones están suficientemente alejados se comportan como partículas localizados que pueden o no poseer carga, así como una estructura interna más compleja. Mientras que al estar cerca, estos interaccionan y se deforman, lo que hace que el problema no pueda resolverse por una simple superposición. Para resolver dicha interacción, una opción es calcular la fuerza entre los solitones y calcular el movimiento relativo entre ellos e interpretar los resultados en términos de las cargas. Dichas fuerzas se pueden calcular directamente de las ecuaciones dependientes del tiempo de los campos o a través de las integrales del tensor de energía-momento; sin embargo no es una tarea fácil.

Por lo anterior no es necesario postular una ley de fuerzas para los solitones, ya que el sentido topológico de estos permite realizar una idea de la física teórica, en la cual dar un entendimiento unificado de la existencia y estructura interna de partículas a la vez de la dinámica e interacción de partículas, la cual no necesita postularse, se obtiene de manera natural.

\subsubsection{Partículas localizadas}

En situaciones donde tenemos un solitón único o solitones suficientemente alejados unos respecto de otros, estos pueden considerarse como objetos localizados con carga. En dichas situaciones se le pueden asociar cantidades como masa y momento a cada uno, utilizando la ecuación de Einstein.

Se calcula la energía del solitón proporcional a la masa del solitón

$$
m=\frac{1}{c^{2}} \int_{-\infty}^{\infty} d x \rho(x),
$$

ya sea en términos de la densidad de energía o del superpotencial. Con lo cual la energía de un solitón en movimiento es $E=\gamma m c^{2}$. Continuando esto, el momento del solitón toma la forma $\vec{p}_{\text {sol }}=\gamma m \vec{v}$, y cuando el solitón esta en reposo $(v=0)$ tenemos que $\vec{p}_{\text {sol }}=\overrightarrow{0}$, así finalmente la energía en reposo es $E_{0}=m c^{2}$. 


\subsubsection{Fuerza de atracción y repulsión}

Cuando los solitones se encuentran alejados la energía de interacción puede obtenerse identificando la fuerza que ejerce un solitón sobre otro a través del la variación de momento. Para un Lagrangiano cuyo potencial sólo depende del campo y no de sus derivadas, el momento en un intervalo semi-infinito está dado por

$$
P=\int T^{10} d x=\int_{-\infty}^{b} \dot{\phi} \phi^{\prime} d x .
$$

Por la segunda ley de Newton sabemos que la fuerza es la derivada temporal del momento no relativista

$$
F=\frac{d P}{d t}=\frac{d}{d t} \int_{-\infty}^{b} \dot{\phi} \phi^{\prime} d x=\int_{-\infty}^{b}\left(\ddot{\phi} \phi^{\prime}+\dot{\phi} \dot{\phi}^{\prime}\right) d x
$$

y usando la ecuación de campo (2.4.1) para integrar tenemos

$$
F=\left[\frac{1}{2}\left(\dot{\phi}^{2}+{\phi^{\prime}}^{2}\right)-V(\phi)\right]_{-\infty}^{b} .
$$

Esto muestra que la fuerza en el intervalo puede ser identificada con la diferencia de presión en los puntos inicial y final. Por lo que es necesario conocer tanto los campos dependientes del tiempo como el potencial. A partir de esto se puede obtener una expresión para la fuerza en términos de la energía de interacción. Esto se logra evaluando el límite superior donde el punto $b$, siendo intermedio y lejano a los solitones, permite utilizar formas asintóticas para los solitones. De aquí es posible obtener una expresión para la energía que dependerá de la separación de los solitones y dará una indicación respecto a la atracción o repulsión que les afecta.

\subsubsection{Colisiones y solitones múltiples}

Estudiar las colisiones de solitones es de gran importancia debido a que siempre buscamos características no sólo de objetos individuales, sino también en interacción con otros similares y distintos. Sin embargo, el tratamiento como objetos puntuales no puede considerarse debido a que los solitones se encuentran cerca, y la interacción es mayor y no lineal, por lo que sufren deformaciones o dispersión. Este tipo de interacción es conveniente estudiarla utilizando solitones múltiples o multisolitón.

Existe una gran variedad de situaciones posibles, cuando las colisiones suceden a altas velocidades la dispersión puede ser muy complicada y la única certeza es que asintóticamente la carga topológica se conserva. Así, cuando un solitón y un antisolitón colisionan, estos pueden aniquilarse y emitir energía en forma ondas de radiación de campos escalares o electromagnéticos, pero la carga total debe conservarse; otra opción es que puedan 
sobrevivir y separarse, emitiendo sólo una pequeña cantidad de radiación. También es posible que parte de la energía puede convertirse en pares solitón-antisolitón. En cualquier caso, los patrones de radiación o interferencia son complicados y en ocasiones sólo se pueden calcular numéricamente. Sin embargo, hay ciertas situaciones donde la cantidad de solitones es igual antes y después de la colisión y sólo una pequeña cantidad de radiación es emitida. Esto ocurre generalmente en una teoría con ecuaciones de Bogomol'nyi, donde los datos iniciales son una configuración del campo cerca de una solución múltiple, pero un poco perturbada por lo que aún es necesario conocer el movimiento relativo entre los solitones.

Obtener soluciones multisolitón implica tratar las interacciones de solitones ya sea, entre objetos del mismo tipo o distinto; si consideremos objetos de distinto tipo debemos considerar que ambas son soluciones de distintas ecuaciones y básicamente estudiar la interacción combinando las soluciones correspondientes. En el caso de objetos del mismo tipo, se pueden considerar solitones múltiples para la misma ecuación. Estas soluciones pueden obtenerse por distintas técnicas y métodos; algunos de los métodos y transformaciones que se pueden aplicar para la solución de la situación antes plateada son: transformaciones de Bäcklund, método de Hirota, método de dispersión inversa (ISM o IST), operadores de Lax, método de traza, sistemas Zakharov-Shabat, método de tanh, etc.

Los métodos mencionados no son aplicables a toda la gama de ecuaciones diferenciables no lineales; algunos son más convenientes que otros según el caso explícito. Dependiendo de la ecuación se pueden presentar soluciones más simples o más complejas; a su vez algunos están limitados en cuanto a la cantidad de dimensiones espaciales que pueden resolver. Además, entre algunos de estos métodos existen relaciones que permiten una generalización de la solución obtenida. Por ejemplo, la transformación de dispersión inversa que es quizá uno de los métodos más generales aunque complicado, está estrechamente relacionado con las transformaciones de Bäcklund, un método más simple aunque no aplicable a todos los sistemas. La monografía de Infeld y Rowlands (1990) presenta un buen panorama general de los métodos mencionados.

\subsection{Transformaciones de Bäcklund}

Una de las maneras más simples de construir soluciones multisolitón es utilizar las transformaciones de Bäcklund; cuando éstas existen permiten (en ciertos casos) la construcción de dichas soluciones de una manera puramente algebraica.

Las transformaciones de Bäcklund son relaciones en el espacio de las variables independientes, dependientes y sus derivadas, de un conjunto de ecuaciones diferenciales parciales; estas relaciones tienen sentido y están bien definidas sólo para dicho conjunto 
de ecuaciones y sus imágenes bajo estas transformaciones. Es decir, las transformaciones de Bäcklund sirven para reducir la integración de una ecuación diferencial parcial lineal o no lineal, a la solución de ecuaciones diferenciales ordinarias, en general de menor orden. También pueden utilizarse para conectar dos distintas soluciones de la misma ecuación o soluciones de distintas ecuaciones. Para soluciones de la misma ecuación algunas veces se le llama autotransformaciones de Bäcklund o transformación invariante de Bäcklund, es decir, si la imagen coincide con el conjunto original, las transformaciones son algunas veces llamadas auto transformaciones de Bäcklund; vid. Taniuti y Nishihara (1983); Stephani (1989); Infeld y Rowlands (1990); Munteanu y Donescu (2005).

Es conocido en la teoría de solitones qué la única ecuación no lineal de Klein-Gordon, qué es integrable es de la forma

$$
\phi_{x t}=V(\phi)
$$

donde se satisface

$$
\frac{\partial^{2} V(\phi)}{\partial \phi^{2}}+k V(\phi)=0,
$$

esta creencia se deriva de dos argumentos distintos: las transformaciones de Bäcklund y la existencia de una cantidad infinita de cantidades conservadas. La ecuación (2.7.1), tiene una autotransformación si y sólo si se satisface (2.7.2); vid. McLaughlin y Scott (1973); Fordy y Gibbons (1980) .

En algunos casos, las soluciones solitónicas permiten obtener soluciones exactas de solitones múltiples partiendo de soluciones simples, a través de la aplicación repetida de este tipo de transformación.

Definición: Un conjunto de relaciones entre $\{t, x, u(t, x)\}$ y $\{T, X, U(T, X)\}$ y las derivadas de $u$ y $U$ es una transformación de Bäcklund (TB) entre $D(u ; t, x)=0$ y $E(U ; T, X)=0$ si:

1. TB es integrable para $U$, si y sólo si $D(u)=0$;

2. TB es integrable para $u$, si y sólo si $E(U)=0$;

3. Dada $u$ tal que $D(u)=0$, TB define $U$ dentro de un conjunto finito de constantes, y $E(U)=0$;

4. Dada $U$ tal que $E(U)=0$, TB define $u$ dentro de un conjunto finito de constantes, y $D(u)=0$.

Comúnmente una transformación de Bäcklund puede ser construida a partir de un mapeo especificando una ecuación de evolución adecuada. Sin embargo, una transformación de Bäcklund no necesita definir unívocamente $u$, inclusive dada $U$, pero no especifica 
ambas $D(u)=0$ y $E(U)=0$; mientras que en un mapeo dada $U$, un mapeo define unívocamente $u$, pero éste no especifica ni $D(u)=0$ ni $E(U)=0$.

Partiendo de esto, consideremos la ecuación de onda en la forma

$$
\left(\frac{1}{c^{2}} \frac{\partial^{2}}{\partial t^{2}}-\frac{\partial^{2}}{\partial x^{2}}\right) \phi=-\frac{\partial V(\phi)}{\partial \phi}=F(\phi)
$$

en término de las características o en este caso coordenadas cono de luz

$$
\xi=\frac{x+c t}{2} \quad \eta=\frac{x-c t}{2} \quad \xi=T \eta
$$

donde $T$ es la inversión temporal, podemos escribir

$$
\phi_{\xi \eta}=\frac{\partial V(\phi)}{\partial \phi}=-F(\phi)
$$

ésta se puede integrar a lo largo de las características

$$
\begin{gathered}
\int\left(\partial_{\xi} \partial_{\eta} \phi=\frac{\partial V(\phi)}{\partial \phi}\right) d \eta \rightarrow \partial_{\xi} \phi=\int \frac{\partial V(\phi)}{\partial \phi} d \eta+r(\xi)=P(\phi) \\
\int\left(\partial_{\xi} \partial_{\eta} \phi=\frac{\partial V(\phi)}{\partial \phi}\right) d \xi \rightarrow \partial_{\eta} \phi=\int \frac{\partial V(\phi)}{\partial \phi} d \xi+s(\eta)=Q(\phi)
\end{gathered}
$$

En general, no hay relación local a lo largo de las características y las derivadas de $\phi$; en este caso se pueden definir los operadores $P(\phi)$ y $Q(\phi)$ de la manera anterior. Sin embargo, al no conocer la relación se puede buscar una relación anterior.

Derivando nuevamente respecto a las características obtenemos

$$
\begin{aligned}
& \partial_{\eta}\left(\partial_{\xi} \phi=P(\phi)\right) \quad \rightarrow \quad \partial_{\eta \xi} \phi=\partial_{\eta} P(\phi)=\partial_{\phi} P \partial_{\eta} \phi \\
& \partial_{\xi}\left(\partial_{\eta} \phi=Q(\phi)\right) \rightarrow \partial_{\xi \eta} \phi=\partial_{\xi} Q(\phi)=\partial_{\phi} Q \partial_{\xi} \phi
\end{aligned}
$$

con esto obtuvimos una relación entre las derivadas de $P$ y $Q$ y recuperamos la ecuación original

$$
\partial_{\xi \eta} \phi=\partial_{\phi} P \partial_{\eta} \phi=\partial_{\phi} Q \partial_{\xi} \phi=-F(\phi)
$$

ahora de esta última relación sustituyendo la definición de $P$ y $Q$

$$
\begin{aligned}
\partial_{\phi} Q \partial_{\xi} \phi & =\partial_{\phi} P \partial_{\eta} \phi \\
\frac{\partial Q}{\partial \phi} P & =\frac{\partial P}{\partial \phi} Q \\
\frac{1}{P} \frac{\partial P}{\partial \phi} & =\frac{1}{Q} \frac{\partial Q}{\partial \phi}
\end{aligned}
$$

e integrando respecto a $\phi$

$$
\int\left(\frac{1}{P} \frac{\partial P}{\partial \phi}=\frac{1}{Q} \frac{\partial Q}{\partial \phi}\right) d \phi \rightarrow \int \frac{d P}{P}=\int \frac{d Q}{Q}
$$




$$
\begin{aligned}
\ln P & =b_{1}+\ln Q \\
P & =e^{b_{1}} Q=a^{2} Q
\end{aligned}
$$

donde $b_{1}$ es una constante de integración y donde $a^{2}>0$, sustituyendo en (2.7.8)

$$
a^{2} Q Q_{\phi}=V_{\phi}
$$

integrando en términos de $P$ obtenemos la relación entre $P, Q$ y $V$

$$
\begin{aligned}
& \int a^{2} Q d Q=\int d V \\
& a^{2} Q^{2}=2\left(V+b_{2}\right)
\end{aligned}
$$

de la definición de $P$ y $Q$ tenemos

$$
\begin{aligned}
Q & = \pm \frac{1}{a}\left[2\left(V+b_{2}\right)\right]^{1 / 2}=\frac{\partial \phi}{\partial \xi} \\
P & = \pm a\left[2\left(V+b_{2}\right)\right]^{1 / 2}=\frac{\partial \phi}{\partial \eta}
\end{aligned}
$$

entonces obtenemos las transformaciones de Bäcklund

$$
\begin{aligned}
\phi_{\xi} & = \pm a \sqrt{2\left(V+b_{2}\right)} \\
\phi_{\eta} & = \pm \frac{\sqrt{2\left(V+b_{2}\right)}}{a}
\end{aligned}
$$

en estos términos, integrando tenemos

$$
\pm \int \frac{d \phi}{\sqrt{2\left(V+b_{2}\right)}}=\int \frac{1}{a} d \eta+\bar{r}(\xi)=\int a d \xi+\bar{s}(\eta)
$$

para considerar la combinación de las integrales características y una constante $b_{3}$ de integración

$$
\pm \int \frac{d \phi}{\sqrt{2\left(V+b_{2}\right)}}=\frac{1}{a} \eta+a \xi+b_{3},
$$

utilizando las coordenadas originales el lado derecho puede escribirse como

$$
\frac{1}{a} \eta+a \xi=\frac{a+a^{-1}}{2}\left[x-\left(\frac{1-a^{2}}{1+a^{2}}\right) c t\right],
$$

de aquí definimos la velocidad y el factor de Lorentz

$$
\begin{aligned}
v & :=c\left(\frac{1-a^{2}}{1+a^{2}}\right) \\
\gamma & :=\frac{a+a^{-1}}{2}
\end{aligned}
$$


como $c$ es la velocidad de la luz y marca un límite físico $|v|<c$, además el signo de sgn $v$ nos da la dirección de movimiento de la onda, invirtiendo la relación de velocidad

$$
a^{2}=\frac{1-\beta}{1+\beta}
$$

ya que ambas $1 \pm \beta>0$. De esta expresión si $v>0$ entonces $|a|>1$; si $u<0$ entonces $|a|<1$, donde identificamos el factor Doppler relativista,

$$
a= \pm \sqrt{\frac{c-v}{c+v}}= \pm \sqrt{\frac{1-\beta}{1+\beta}}=\gamma(1-\beta),
$$

en este caso el sgn $a$ no determina el valor de $v$, y utilizando ésta el factor de Lorentz queda

$$
\gamma= \pm \frac{1}{\sqrt{1-\beta^{2}}}
$$

y el $\operatorname{sgn} \gamma$ esta determinado por el $\operatorname{sgn} a$, donde la relación de velocidades $\beta=v / c$. Finalmente con estas definiciones podemos escribir

$$
a \xi+\frac{1}{a} \eta= \pm \gamma(x-v t)= \pm \gamma(x-c \beta t)= \pm \zeta
$$

e invirtiendo obtenemos la solución

$$
\phi=K^{-1}\left( \pm \zeta+b_{3}\right)
$$

donde

$$
K= \pm \int \frac{d \phi}{\sqrt{2\left(V+b_{2}\right)}}
$$

\subsubsection{Principio de superposición no lineal}

Consideremos la superposición de dos soluciones de $(2.7 .5) \phi^{(0)}$ y $\phi^{(1)}$; para cada una de éstas la transformación de Bäcklund

$$
\begin{aligned}
\phi_{\xi}^{(i)} & =\int G\left(\phi^{(i)}\right) d \eta \\
\phi_{\eta}^{(i)} & =\int G\left(\phi^{(i)}\right) d \xi
\end{aligned}
$$

es posible proponer un principio de superposición

$$
\begin{aligned}
\phi_{\xi}^{(1)}+\alpha_{1} \phi_{\xi}^{(0)} & =\int\left\{G\left(\phi^{(1)}\right)+\alpha_{1} G\left(\phi^{(0)}\right)\right\} d \eta=P\left(\phi^{(1)}, \phi^{(0)}\right) \\
\phi_{\eta}^{(1)}+\alpha_{2} \phi_{\eta}^{(0)} & =\int\left\{G\left(\phi^{(1)}\right)+\alpha_{2} G\left(\phi^{(0)}\right)\right\} d \xi=Q\left(\phi^{(1)}, \phi^{(0)}\right)
\end{aligned}
$$


donde $\alpha_{1}$ y $\alpha_{2}$ son constantes, derivando cruzado

$$
\begin{aligned}
\left(\phi_{\xi}^{(1)}+\alpha_{1} \phi_{\xi}^{(0)}\right)_{\eta} & =G\left(\phi^{(1)}\right)+\alpha_{1} G\left(\phi^{(0)}\right)=P_{\phi^{(1)}} \phi_{\eta}^{(1)}+P_{\phi^{(0)}} \phi_{\eta}^{(0)} \\
\left(\phi_{\eta}^{(1)}+\alpha_{2} \phi_{\eta}^{(0)}\right)_{\xi} & =G\left(\phi^{(1)}\right)+\alpha_{2} G\left(\phi^{(0)}\right)=Q_{\phi^{(1)}} \phi_{\xi}^{(1)}+Q_{\phi^{(0)}} \phi_{\xi}^{(0)}
\end{aligned}
$$

y combinando las ecuaciones (2.7.26) obtenemos las condiciones

$$
\begin{aligned}
P Q_{\phi^{(1)}}-Q P_{\phi^{(1)}} & =-\left(\alpha_{1}-\alpha_{2}\right) G^{(0)} \\
P Q_{\phi^{(0)}}-Q P_{\phi^{(0)}} & =\left(\alpha_{1}-\alpha_{2}\right) G^{(1)} \\
\alpha_{1} Q_{\phi^{(1)}}-Q_{\phi^{(0)}} & =0 \\
\alpha_{2} P_{\phi^{(1)}}-P_{\phi^{(0)}} & =0 .
\end{aligned}
$$

De las últimas dos ecuaciones tenemos

$$
\begin{aligned}
& P=P\left(v:=\phi^{(1)}+\alpha_{2} \phi^{(0)}\right) \\
& Q=Q\left(u:=\phi^{(1)}+\alpha_{1} \phi^{(0)}\right)
\end{aligned}
$$

asumiendo $\alpha_{1} \neq \alpha_{2}$, derivando (2.7.28a) respecto a $\phi^{(1)}$

$$
P Q_{u u}-Q P_{v v}=0
$$

y derivando $(2.7 .28 \mathrm{~b})$ respecto $\phi^{(0)}$

$$
\alpha_{1}^{2} P Q_{u u}-\alpha_{2}^{2} Q P_{v v}=0
$$

de estas ecuaciones obtenemos $\alpha_{1}^{2}=\alpha_{2}^{2}$, de ser iguales $\alpha_{1}$ y $\alpha_{2}$ tenemos el principio de superposición usual.

Entonces la transformación de Bäcklund puede escribirse como

$$
\begin{aligned}
\left(\phi^{(1)}+\alpha_{1} \phi^{(0)}\right)_{\xi} & =P\left(\phi^{(1)}+\alpha_{2} \phi^{(0)}\right) \\
\left(\phi^{(1)}+\alpha_{2} \phi^{(0)}\right)_{\eta} & =Q\left(\phi^{(1)}+\alpha_{1} \phi^{(0)}\right) .
\end{aligned}
$$

Aquí en general, $\phi^{(0)}$ no necesita ser solución de $(2.7 .5)$, solamente es necesario que $\phi^{(0)}$ sea una función suficientemente suave de $\xi$ y $\eta$, en cuyo caso la condición de integrabilidad es $\alpha_{1}=\alpha_{2}$. Y en vista de lo anterior, dadas $\alpha_{1}, \alpha_{2}$ y $\phi^{(0)}$ podemos obtener $\phi^{(1)}$ de las ecuaciones (2.7.32). Como las soluciones se mantienen para dos soluciones arbitrarias, es posible obtener más soluciones aplicando sucesivamente las transformación.

Así, podemos escribir

$$
\begin{aligned}
\phi^{(0)} & =0 \\
\phi^{(1)} & =B_{1} \phi^{(0)} \\
\phi^{(2)} & =B_{2} \phi^{(1)}=B_{2} B_{1} \phi^{(0)} \\
& \vdots \\
\phi^{(n)} & =B_{n} \phi^{(n-1)}=B_{n} B_{-1} \cdots B_{1} \phi^{(0)}
\end{aligned}
$$


donde $B_{j}$ es la transformación de que actúa como un operador incremental, que no es necesariamente conmutativo

$$
B_{3} B_{2} \phi^{(1)} \neq B_{2} B_{3} \phi^{(1)}
$$

Sin embargo, las constantes de integración $b_{i}$ en (2.7.24) son arbitrarias, y pueden elegirse apropiadamente tal que

$$
B_{3}\left(a_{3}, b_{3}\right) B_{2}\left(a_{2}, b_{2}\right) \phi^{(1)}=B_{2}\left(a_{2}, b_{2}^{\prime}\right) B_{3}\left(a_{3}, b_{3}^{\prime}\right) \phi^{(1)}
$$

esto puede representarse gráficamente por un diagrama de Lamb

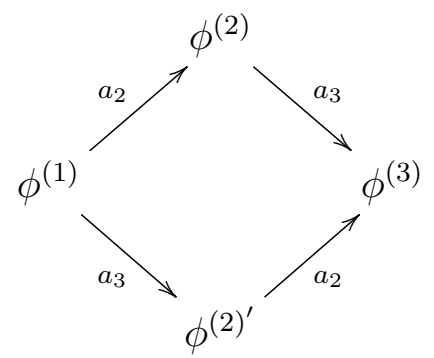

Extendiendo el diagrama de Lamb a tercer orden

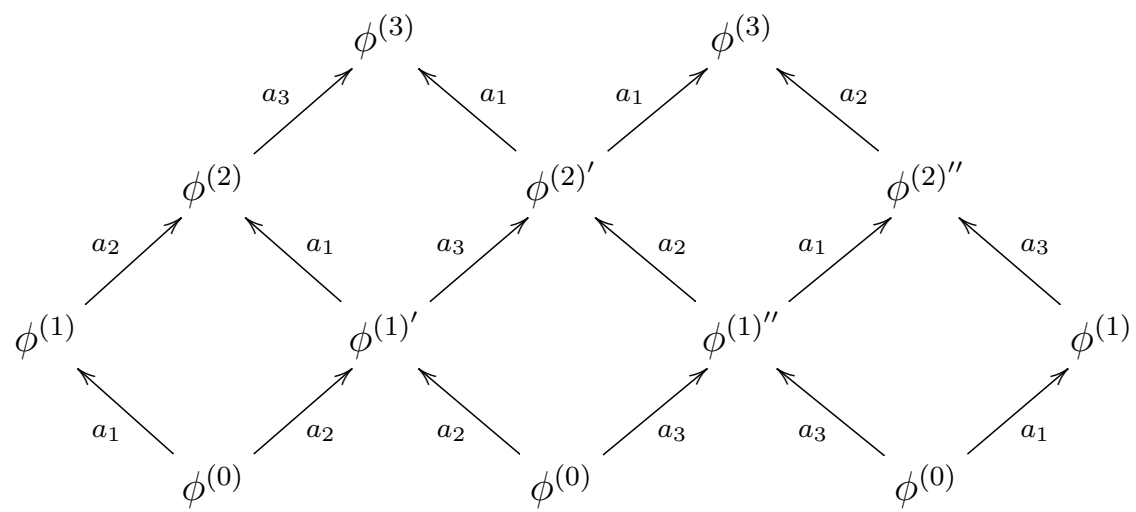

consideremos las transformaciones

$$
\begin{aligned}
\phi^{(1)} & =B_{1} \phi^{(0)} \\
\phi^{(2)} & =B_{2} \phi^{(0)}
\end{aligned}
$$

y el principio de superposición no lineal

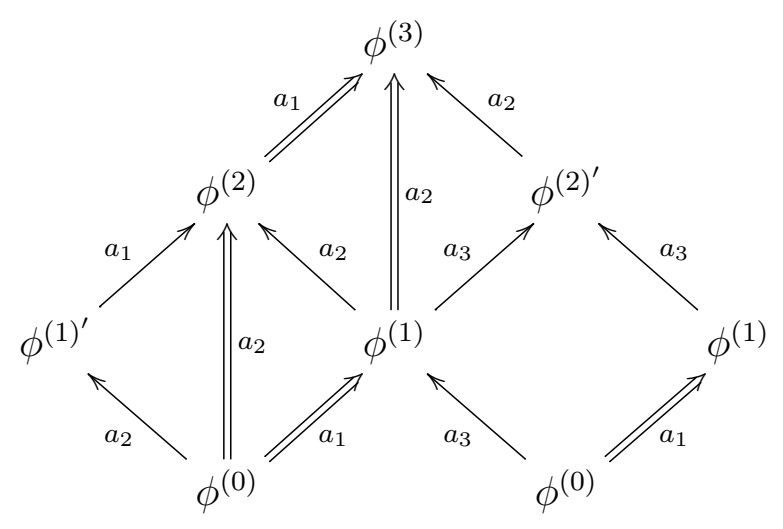


Como consecuencia

$$
\phi^{(3)}=B_{2} \phi^{(1)}=B_{1} \phi^{(2)}=B_{2} B_{1} \phi^{(0)}=B_{1} B_{2} \phi^{(0)}
$$

Esto tiene la representación

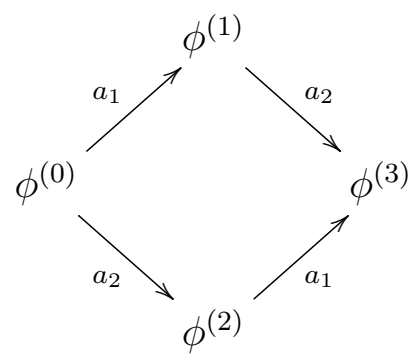

representa cuatro transformaciones, que combinadas adecuadamente permiten obtener una expresión puramente algebraica, es decir, sin incluir diferenciales, la cual se denomina teorema de permutabilidad de Bianchi o teorema de adición de transformaciones de Bäcklund; vid. Felsager (1983); Taniuti y Nishihara (1983).

Básicamente la aplicación sucesiva de las transformaciones sobre un juego de soluciones permite eliminar las derivadas y obtener una relación algebraica de entre éstas. Derivado de lo que Bianchi notó, si las transformaciones se reformulan como operadores dependientes de $\phi_{0}$ (conocida), se pueden obtener dos soluciones adicionales

$$
\begin{aligned}
& \phi_{1}=B_{a_{1}}\left[\phi_{0}\right] \\
& \phi_{2}=B_{a_{2}}\left[\phi_{0}\right]
\end{aligned}
$$

y que la aplicación sucesiva, genera una tercera solución

$$
\begin{aligned}
& \phi_{3}=B_{a_{2}}\left[\phi_{1}\right]=B_{a_{2}}\left[B_{a_{1}}\left[\phi_{0}\right]\right] \\
& \phi_{3}=B_{a_{1}}\left[\phi_{2}\right]=B_{a_{1}}\left[B_{a_{2}}\left[\phi_{0}\right]\right]
\end{aligned}
$$

donde además las transformaciones de Bäcklund conmutan.

\subsection{Modelo sine-Gordon}

El modelo de sine-Gordon ( $\mathrm{sG}$ ) es utilizado para estudiar muchos fenómenos en distintas áreas de conocimiento; y juega un papel notable en geometría diferencial, óptica no lineal, física de plasmas, superconductividad, axiones, física de partículas, dislocación de cristales, ondas splay, líneas de trasmisión, etc.; vid. Rubinstein (1970); Barone et al. (1971); Dodd et al. (1982); Rajaraman (1982); Kumar y Thomas (1988). 


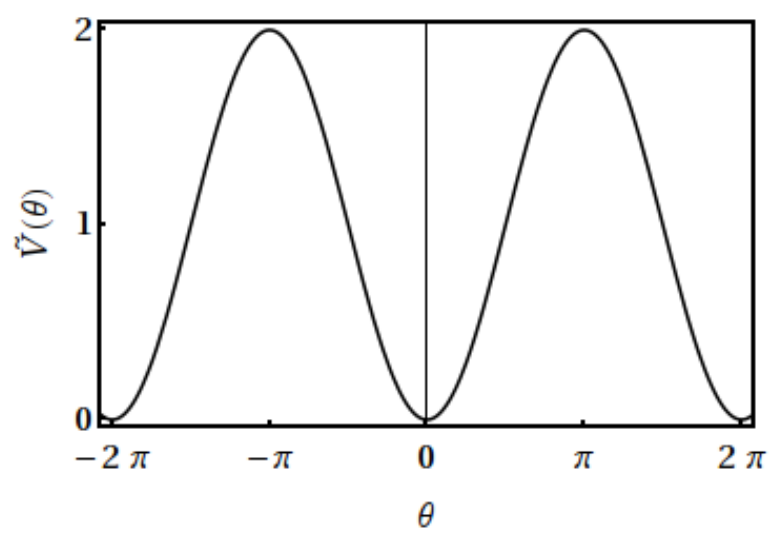

Figura 2.8.1: Potencial $\tilde{V}(\theta)=(1-\cos \theta)=\tilde{V}(\theta)$.

\subsubsection{Ecuación de sine-Gordon}

La ecuación de sine-Gordon fue introducida originalmente por J. Edmond Bour (1862) como ecuación de Gauss-Codazzi para estudiar superficies de curvatura negativa constante, y posteriormente redescubierta por Frenkel y Kontorova (1939) como un límite continuo para dislocaciones en estado sólido. El nombre actual de debe a Martin Kruskal, por un juego de palabras debido a su semejanza respecto a la ecuación de Klein-Gordon (4.0.2). Además, se puede interpretar la ecuación de Klein-Gordon como la aproximación lineal de la ecuación de sine-Gordon.

Consideremos primero una Lagrangiana de la forma

$$
\mathcal{L}\left(\phi, \partial_{\mu} \phi\right)=\frac{1}{2} \partial_{\mu} \phi \partial^{\mu} \phi-V(\phi)
$$

cuyo potencial está dado por

$$
V_{\mathrm{sG}}(\phi)=\frac{m^{4}}{\lambda}\left[1-\cos \left(\frac{\sqrt{\lambda}}{m} \phi\right)\right],
$$

simétrico y periódico (Figura 2.8.1). El potencial es conocido por el nombre de la ecuación, potencial de sine-Gordon.

Considerando el potencial completo, la densidad Lagrangiana de sine-Gordon es

$$
\mathcal{L}_{\mathrm{sG}}(t, x)=\frac{1}{2}\left(\partial_{\mu} \phi\right)\left(\partial^{\mu} \phi\right)-\frac{m^{4}}{\lambda}\left[1-\cos \left(\frac{\sqrt{\lambda}}{m} \phi\right)\right]
$$

y la ecuación de onda resulta en

$$
\square \phi+\frac{m^{3}}{\sqrt{\lambda}} \sin \left(\frac{\sqrt{\lambda}}{m} \phi\right)=0 .
$$


Para simplificar la notación, redefinimos las variables como

$$
\tilde{x}=m x, \quad \tilde{t}=m t, \quad \theta=\frac{\sqrt{\lambda}}{m} \phi,
$$

podemos reescribir las ecuaciones (2.8.3) y (2.8.4) de una manera más simple

$$
\begin{aligned}
& \tilde{\mathcal{L}}_{\mathrm{SG}}(\tilde{t}, \tilde{x})=\frac{m^{4}}{\lambda} {\left[\frac{1}{2}\left(\tilde{\partial}_{\mu} \theta\right)\left(\tilde{\partial}^{\mu} \theta\right)-(1-\cos \theta)\right] } \\
& \tilde{\square} \theta+\sin \theta=0 .
\end{aligned}
$$

Ambas ecuaciones (2.8.6) y (2.8.7) tienen las simetrías $P, T$ y $C$, además de ser invariantes ante transformaciones de Lorentz.

El tipo de solución a obtener está determinada por los puntos críticos y ceros del potencial (2.8.2), así

$$
\begin{aligned}
V_{\mathrm{sG}}(\theta) & =\frac{m^{4}}{\lambda}(1-\cos \theta)=0 \\
\frac{d}{d \theta} V_{\mathrm{sG}}(\theta) & =\frac{m^{4}}{\lambda} \sin \theta=0 .
\end{aligned}
$$

De estos, la forma periódica del potencial $V(\theta)$ tiene mínimos que son ceros en cada punto $\bar{\theta}_{n}=2 n \pi$, mientras que tiene máximos en los puntos $\theta=(2 n+1) \pi$, con $n \in \mathbb{Z}$. A diferencia de la ecuación de Klein-Gordon, aquí hay más de un punto critico con lo que una solución puede conectar distintos puntos críticos; es decir, podemos tener soluciones topológicas y no topológicas. La modularidad del potencial da lugar a sectores topológico de ancho $2 \pi$, cuando $\tilde{x} \rightarrow \pm \infty$, lo que da las condiciones de frontera. Estas condiciones muestran los solitones tipo kink de la siguiente manera: el campo $\theta \rightarrow \bar{\theta}_{n}$ cuando $\tilde{x} \rightarrow-\infty$, y $\theta \rightarrow \bar{\theta}_{n+1}$ cuando $\tilde{x} \rightarrow \infty$; o $\theta \rightarrow \bar{\theta}_{n-1}$ cuando $\tilde{x} \rightarrow \infty$, y $\theta \rightarrow \bar{\theta}_{n}$ cuando $\tilde{x} \rightarrow-\infty$, respectivamente soluciones tipo kink y antikink.

La carga topológica tiene una corriente asociada (2.4.14) que no es dinámica

$$
J^{0}:=\frac{1}{2 \pi} \partial_{\tilde{x}} \theta
$$

y la carga (2.4.17) es simplemente

$$
Q=\frac{1}{2 \pi}\left(\theta_{+}-\theta_{-}\right)
$$

como la Lagrangiana (2.8.6) es invariante ante un corrimiento de $2 \pi$ del campo, podemos tomar $\theta_{ \pm}=2 \pi$ y $\theta_{\mp}=0$, para $\tilde{x} \rightarrow \pm \infty$ según sea el caso.

Podemos obtener además el superpotencial $\tilde{W}(\theta)$ que toma la forma (Figura 2.8.2)

$$
W_{\mathrm{k}, \overline{\mathrm{k}}}(\theta)=\frac{m^{3}}{\lambda} \tilde{W}_{\mathrm{k}, \overline{\mathrm{k}}}(\theta)=\mp 4 \frac{m^{3}}{\lambda} \cos \frac{\theta}{2}
$$

podemos ver que al igual que el potencial el superpotencial es periódico. 

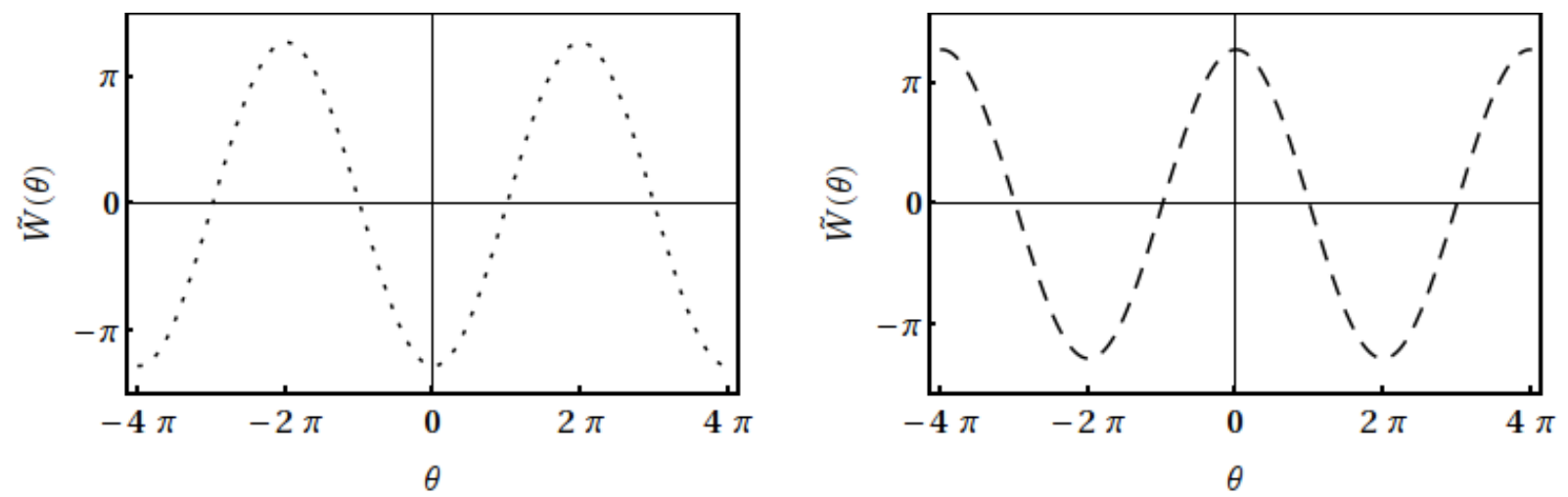

Figura 2.8.2: Los superpotenciales $\tilde{W}(\theta)=\mp 4 \cos \frac{\theta}{2}$ al igual que el potencial $\tilde{V}(\theta)$ son periódicos.
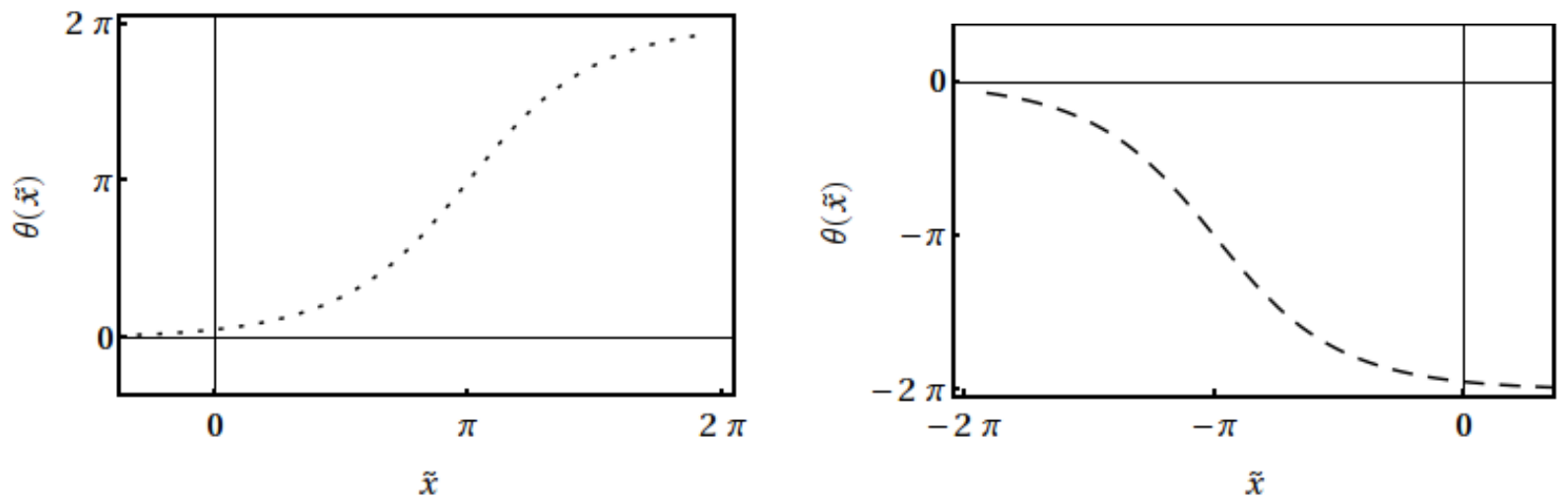

Figura 2.8.3: Solución tipo kink centrada en $\tilde{x}_{o}=\pi$, solución tipo antikink centrada en $\tilde{x}_{o}=-\pi$.

\subsubsection{Solitones de sine-Gordon}

Resolviendo la ecuación (2.8.7), para una solución estática

$$
\frac{\partial^{2} \theta}{\partial \tilde{x}^{2}}=\sin \theta, \quad \bmod 2 \pi
$$

para integrar, multiplicando la ecuación con el factor integrante $\partial \theta / \partial \tilde{x}$ a ambos lados, podemos escribir

$$
\frac{\partial \theta}{\partial \tilde{x}}=2 \sin \left(\frac{\theta}{2}\right)
$$

e integrando llegamos a la solución implícita

$$
\begin{aligned}
& \int_{\tilde{x}_{0}}^{\tilde{x}} \mathrm{~d} \tilde{x}= \pm \int_{\theta_{0}}^{\theta} \frac{\mathrm{d} \theta}{2 \sin (\theta / 2)} \\
& \tilde{x}-\tilde{x}_{0}= \pm \ln \left|\tan \frac{\theta}{4}\right| .
\end{aligned}
$$

De aquí despejando el campo tenemos 


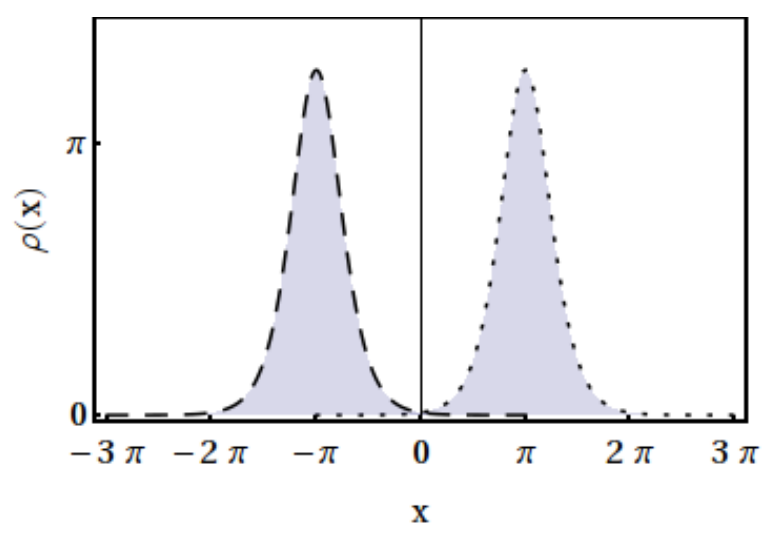

Figura 2.8.4: Energía calculada utilizando la densidad de energía $\tilde{\rho}(\tilde{x})$, tanto para el solitón y antisolitón.

$$
\theta(\tilde{x})= \pm 4 \arctan \left\{\exp \left[ \pm\left(\tilde{x}-\tilde{x}_{0}\right)\right]\right\}
$$

Aquí, los signos designan la carga topológica, determinando el sentido de tornillo en el cual se desplaza la onda. Se identifican dos tipos de soluciones con signos iguales para el solitón o kink ${ }^{5}$ (Figura 2.8.3a) y signos distintos para el antisolitón o antikink (Figura 2.8.3b). Es decir, una simetría del campo tanto de conjugación de la carga como de paridad y definen la relación entre kink y antikink. De las cuatro posibles soluciones resultan dos kinks y dos antikinks pero lo hacen en sectores topológicos adyacentes. La solución tipo kink, va de $\bar{\theta}=2 \pi N$ a $\bar{\theta}=2 \pi(N+1)$ y tiene una carga $Q=+1$; mientras que la solución tipo antikink, va de $\bar{\theta}=2 \pi N$ a $\bar{\theta}=2 \pi(N-1)$ y tiene $Q=-1$.

La solución dinámica la obtenemos aplicando un boost de Lorentz a (2.8.15); esto es, reemplazando $\left(\tilde{x}-\tilde{x}_{o}\right) \rightarrow \gamma\left(\tilde{x}-\tilde{x}_{o}-v \tilde{t}\right)$, donde $\gamma=\left(1-v^{2} / c^{2}\right)^{-1 / 2}$ es el factor de Lorentz y $v \leq c$ la velocidad de la onda, con lo cual

$$
\theta(\tilde{t}, \tilde{x})= \pm 4 \arctan \left\{\exp \left[ \pm \gamma\left(\tilde{x}-\tilde{x}_{o}-v \tilde{t}\right)\right]\right\}
$$

La energía la obtenemos de la cota de Bogomol'nyi (2.5.12), ya sea mediante la densidad de energía (Figura 2.8.4)

$$
\rho(\tilde{x})=\frac{m^{4}}{\lambda} \tilde{\rho}(\tilde{x})=4 \frac{m^{4}}{\lambda} \operatorname{sech}^{2}\left(\tilde{x}-\tilde{x}_{0}\right)
$$

\footnotetext{
${ }^{5}$ Si bien, el kink (antikink) es una función diferente $\phi(x)= \pm(m / \sqrt{\lambda}) \tanh \left[(m / \sqrt{\lambda})\left(x-x_{0}\right)\right]$, ambas tienen la misma forma, por lo cual la llamaremos tipo kink (antikink) o simplemente kink (antikink).
} 
o utilizando el superpotencial (2.8.11), así

$$
\begin{aligned}
E_{\mathrm{k}} & =\int_{-\infty}^{\infty} \frac{m^{3}}{\lambda}\left[\frac{1}{2}\left(\frac{\partial \theta}{c \partial \tilde{t}}\right)^{2}+\frac{1}{2}\left(\frac{\partial \theta}{\partial \tilde{x}}\right)^{2}+(1-\cos \theta)\right] \mathrm{d} \tilde{x} \\
& =\left|\tilde{W}\left(\theta_{+}\right)-\tilde{W}\left(\theta_{-}\right)\right|^{2 \pi}=\frac{8 m^{3}}{\lambda}, \\
& =\left[-\frac{4 m^{3}}{\lambda} \cos \left(\frac{\theta}{2}\right)\right]_{0}^{2 \pi}=\frac{1}{}
\end{aligned}
$$

esto puede reescribirse en forma relativista utilizando la ecuación de Einstein, $E=\gamma m c^{2}$, con lo que podemos identificar la masa del kink como $m_{\mathrm{k}}=8 \mathrm{~m}^{3} / \lambda$. Y el momento del kink $p_{\mathrm{k}}=\gamma m_{\mathrm{k}} v$, cuando el solitón esta en reposo $(v=0)$ tenemos $p_{\mathrm{k}}=0$, y la energía en reposo es $E_{0 \mathrm{k}}=m_{\mathrm{k}} c^{2}$.

\subsubsection{Transformación de Bäcklund}

Se han mostrado dos soluciones a la ecuación de sine-Gordon; el kink y antikink (2.8.16) ambos solitones topológicos. De hecho, el sistema permite un tercer tipo, un solitón no topológico conocido como breather, doblete o bión. Estas tres soluciones no son las únicas soluciones de la ecuación, de hecho la ecuación permite solitones múltiples; es decir, soluciones 2-solitón, 3-solitón, 4-solitón, etc. Para obtener soluciones como estas últimas, se requiere aplicar métodos inherentes a la no linealidad de las ecuaciones, como los ya mencionados. En particular para la ecuación de sine-Gordon un método simple son las transformaciones Bäcklund, que fueron encontradas por Albert V. Bäcklund en 1875.

Reescribiendo la ecuación en coordenadas de cono de luz, y considerando la compatibilidad del intercambio de las derivadas, la ecuación (2.8.7) se puede escribir como

$$
\partial_{\eta} \partial_{\xi} \tilde{\varphi}=\sin \tilde{\varphi}
$$

Las transformaciones de Bäcklund para la ecuación de sine-Gordon son

$$
\begin{aligned}
& \frac{1}{2} \partial_{\xi}(\tilde{\varphi}+\tilde{\phi})=a \sin \left[\frac{1}{2}(\tilde{\varphi}-\tilde{\phi})\right] \\
& \frac{1}{2} \partial_{\eta}(\tilde{\varphi}-\tilde{\phi})=\frac{1}{a} \sin \left[\frac{1}{2}(\tilde{\varphi}+\tilde{\phi})\right]
\end{aligned}
$$

donde $a$ es un parámetro real, que se determina a partir de la velocidad del solitón. Utilizando la ecuación (2.8.19) en términos de una combinación lineal de las soluciones $\tilde{\phi}(\xi, \eta)$ y $\tilde{\varphi}(\xi, \eta)$, podemos notar

$$
\partial_{\eta} \partial_{\xi}(\tilde{\varphi} \pm \tilde{\phi})=\sin \tilde{\varphi} \pm \sin \tilde{\phi} \neq \sin (\tilde{\varphi} \pm \tilde{\phi})
$$

lo que muestra que la combinación lineal no es solución; salvo en el caso de una solución trivial no solitónica $\tilde{\phi}=0$. 
La forma explicita de $\tilde{\varphi}$ la obtenemos al integrar las ecuaciones (2.8.20), y utilizando éstas podemos obtener la solución de (2.8.19), y considerando $\tilde{\phi}(\xi, \eta)=0$ tenemos

$$
\begin{aligned}
& \frac{1}{2} \partial_{\xi}(\tilde{\varphi})=a \sin \left(\frac{\tilde{\varphi}}{2}\right) \\
& \frac{1}{2} \partial_{\eta}(\tilde{\varphi})=\frac{1}{a} \sin \left(\frac{\tilde{\varphi}}{2}\right) .
\end{aligned}
$$

Que podemos integrar escribiendo

$$
\frac{\mathrm{d}(\tilde{\varphi} / 2)}{\sin (\tilde{\varphi} / 2)}=\frac{\mathrm{d} \eta}{a}=a \mathrm{~d} \xi
$$

y entonces

$$
\ln \tan \left(\frac{\tilde{\varphi}}{4}\right)=a \xi+\frac{1}{a} \eta+\delta
$$

donde $\delta$ es una constante de integración, finalmente obtenemos

$$
\tilde{\varphi}=4 \arctan \left[\exp \left(a \xi+\frac{1}{a} \eta+\delta\right)\right],
$$

y regresando a las coordenadas originales $(\tilde{t}, \tilde{x})$ y utilizando (2.7.19)

$$
\begin{aligned}
\tilde{\varphi} & =4 \arctan \left\{\exp \left[\frac{a+a^{-1}}{2}\left(\tilde{x}-\frac{1-a^{2}}{1+a^{2}} c \tilde{t}-\delta\right)\right]\right\} \\
& =4 \arctan \{\exp [ \pm \gamma(\tilde{x}-v \tilde{t}+\delta)]\}
\end{aligned}
$$

donde $\beta=v / c$, y el signo de $a$ determinar el signo de $\gamma$, a la vez el kink o antikink.

\subsubsection{Teorema de permutabilidad de Bianchi}

Para obtener el teorema de permutabilidad, las transformaciones de Bäcklund para sineGordon se pueden escribir como

$$
\begin{aligned}
\partial_{\xi}\left(\phi_{i}+\phi_{j}\right) & =a_{k} \sin \left[\frac{1}{2}\left(\phi_{i}-\phi_{j}\right)\right] \\
\partial_{\eta}\left(\phi_{i}-\phi_{j}\right) & =\frac{1}{a_{k}} \sin \left[\frac{1}{2}\left(\phi_{i}+\phi_{j}\right)\right]
\end{aligned}
$$

donde $\phi_{i}$ y $\phi_{j}$ son soluciones de la ecuación. Considerando combinaciones de las soluciones sobre alguna de (2.8.26), podemos obtener la relación

$$
\tan \left[\frac{\phi_{3}-\phi_{0}}{4}\right]=\frac{a_{2}+a_{1}}{a_{2}-a_{1}} \tan \left[\frac{\phi_{2}-\phi_{1}}{4}\right] .
$$

Esta relación es un principio de superposición no-lineal para $\phi_{0}, \phi_{1}, \phi_{2}$ y $\phi_{3}$; principio que puede representarse gráficamente como 


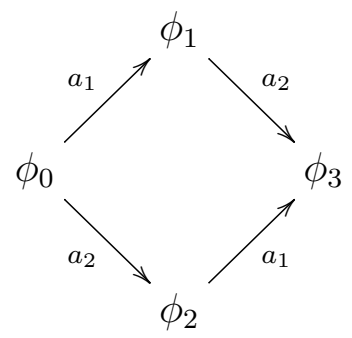

estos diagramas se conocen como diagramas de Lamb o de Bianchi.

Finalmente la expresión (2.8.27) nos permite obtener una nueva solución $\phi_{3}$, en términos de otras tres soluciones conocidas $\phi_{0}, \phi_{1}$ y $\phi_{2}$,

$$
\phi_{3}=\phi_{0}+4 \arctan \left[\frac{a_{1}+a_{2}}{a_{1}-a_{2}} \tan \left(\frac{\phi_{1}-\phi_{2}}{4}\right)\right],
$$

y donde los parámetros $a_{1}$ y $a_{2}$ pueden obtenerse, a partir de las transformaciones (2.8.26), ya que en éstas todas las funciones $\phi_{i}$ son conocidas, $i=0,1,2$.

\subsubsection{Solución multisolitón}

La expresión (2.8.29) nos permite obtener la solución multisolitón partiendo de soluciones simples, mediante un proceso de iteración. Por ejemplo, partiendo de dos soluciones triviales y un solitón simple podemos recuperar el solitón simple; partiendo de una solución trivial y dos solitones simples, obtenemos un solitón doble o 2-solitón; partiendo de tres solitones simples, obtenemos un 3-solitón. Progresivamente de esta manera podemos obtener soluciones del grado que queramos o que permita el sistema. El modelo de sineGordon permite soluciones de cualquier grado, ya que es un sistema totalmente integrable y que permite una cantidad infinita de constantes de integración.

Para nuestros propósitos consideremos la solución 2-solitón. Esto es, en (2.8.29) tomemos las funciones $\phi_{0}=0, \phi_{1}=\phi_{\mathrm{S} 1}$ y $\phi_{2}=\phi_{\mathrm{S} 2}$; donde $\phi_{\mathrm{S} i}$ son soluciones tipo kink o antikink. Entonces la función $\phi_{3}$ esta dada por

$$
\phi_{3}=4 \arctan \left[\frac{a_{1}+a_{2}}{a_{1}-a_{2}} \tan \left(\frac{\phi_{\mathrm{S} 1}-\phi_{\mathrm{S} 2}}{4}\right)\right]
$$

y las transformaciones (2.8.26) se reducen a

$$
\partial_{\xi} \phi_{\mathrm{S} i}=2 a_{i} \sin \left(\frac{1}{2} \phi_{\mathrm{S} i}\right) \quad \partial_{\eta} \phi_{\mathrm{S} i}=2 a_{i}^{-1} \sin \left(\frac{1}{2} \phi_{\mathrm{S} i}\right),
$$

donde $i=1,2$; con esto los parámetros $a_{i}$ para ambas ecuaciones están dados por la relación $(2.7 .21)$

$$
a_{i}= \pm \sqrt{\frac{1-\beta_{i}}{1+\beta_{i}}}=\gamma_{i}\left(1-\beta_{i}\right)
$$



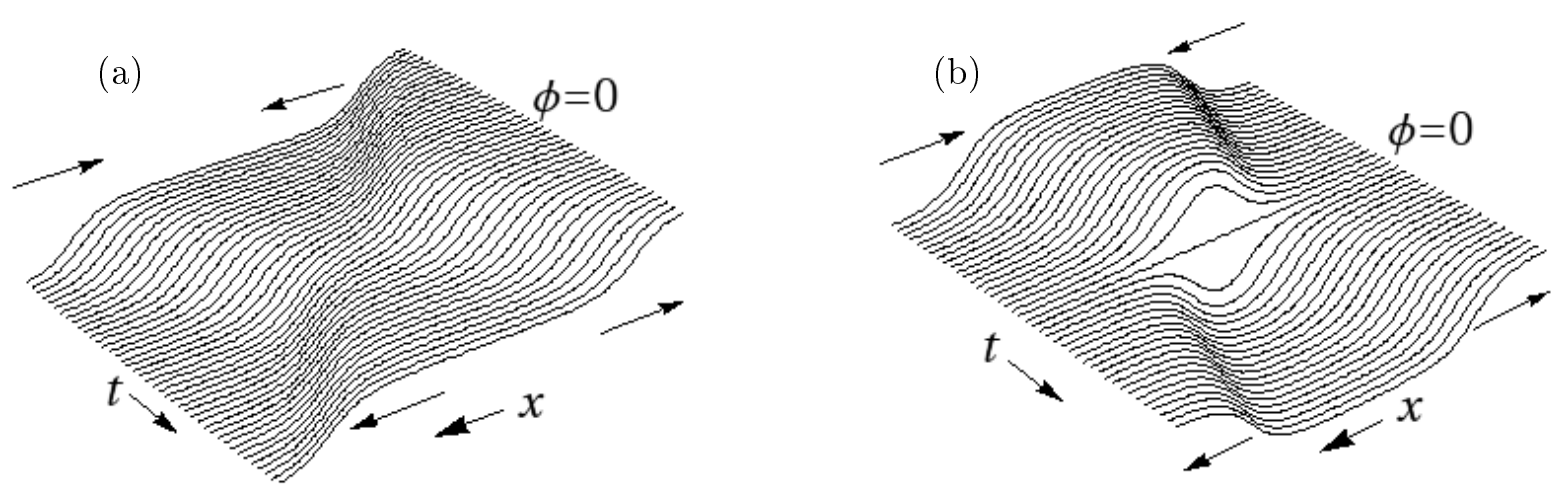

Figura 2.8.5: (a) Solitón kink-kink (antikink-antikink) y (b) Solitón kink-antikink.

Para obtener una forma general de $\phi_{3}$ escribamos los solitones como sigue

$$
\phi_{\mathrm{S} i}=4 \arctan \left(\mathrm{e}^{\zeta_{i}}\right)
$$

donde $\zeta_{i}= \pm \gamma_{i}\left(\tilde{x}_{i}-\tilde{x}_{i 0}-v_{i} \tilde{t}_{i}\right), \gamma_{i}=\left(1-v_{i}^{2}\right)^{-1 / 2}$ y hemos tomado $\tilde{t}_{0 i}=0$, con esto

$$
\phi_{3}=4 \arctan \left\{\mathbb{B} \frac{\sinh \left[\left(\zeta_{1}-\zeta_{2}\right) / 2\right]}{\cosh \left[\left(\zeta_{1}+\zeta_{2}\right) / 2\right]}\right\}
$$

donde

$$
\mathbb{B}=\frac{a_{1}+a_{2}}{a_{1}-a_{2}} .
$$

El comportamiento asintótico de $\phi_{3}$; debe recuperar dos solitones independientes, tanto en un pasado lejano como en un futuro distante a la colisión. Dos soluciones continuas pueden obtenerse de esto, una como la colisión de dos solitones de la misma especie kink-kink (antikink-antikink) y otra con solitones de distinta especie kink-antikink (Figura 2.8.5).

\subsubsection{Colisión kink-kink (antikink-antikink)}

Para esta solución 2-solitón, utilizaremos la formula (2.8.30), en la cual utilizamos

$$
\begin{aligned}
& \phi_{1}=4 \arctan \left\{\exp \left[-\gamma_{1}\left(\tilde{x}_{1}-\tilde{x}_{10}-v_{1} \tilde{t}_{1}\right)\right]\right\}=\phi_{\text {antikink }}=\phi_{\overline{\mathrm{k}}} \\
& \phi_{2}=4 \arctan \left\{\exp \left[\gamma_{2}\left(\tilde{x}_{2}-\tilde{x}_{20}-v_{2} \tilde{t}_{2}\right)\right]\right\}=\phi_{\text {kink }}=\phi_{\mathrm{k}}
\end{aligned}
$$

un kink $\phi_{2}$ que viaja de izquierda a derecha y un antikink $\phi_{1}$ en dirección opuesta. Por simplicidad, tomaremos ambos solitones con la misma masa $m_{1}=m_{2}=m$ y rapidez, así $v_{1}=-v_{2}=v>0$ y con posiciones iniciales simétricas $-x_{10}=x_{20}=x_{0}>0$, con estas condiciones es más simple escribir

$$
\begin{aligned}
\zeta_{1} & =-\gamma\left(\tilde{x}+\tilde{x}_{0}-v \tilde{t}\right) \\
\zeta_{2} & =\gamma\left(\tilde{x}-\tilde{x}_{0}+v \tilde{t}\right)
\end{aligned}
$$


y $\gamma_{1}=\gamma_{2}=\gamma=\left(1-\beta^{2}\right)^{-1 / 2}$, con $\beta=v / c$ en (2.8.34). Sustituyendo en (2.8.35) tenemos $\mathbb{B}_{\mathrm{kk}}=-v / c=-\left|\mathbb{B}_{\mathrm{kk}}\right|$ y la solución

$$
\phi_{\mathrm{kk}}=4 \arctan \left\{\frac{v \sinh [\gamma \tilde{x}]}{c \cosh \left[\gamma\left(v \tilde{t}-\tilde{x}_{0}\right)\right]}\right\}=-\phi_{\overline{\mathrm{kk}}},
$$

que en el tiempo representa la colisión dos kinks (Figura 2.8.5a).

En este caso el comportamiento asintótico de $\phi_{\mathrm{kk}}$, lo podemos evidenciar al escribir

$$
\begin{aligned}
\tan \left(\frac{\varphi}{4}\right) & =\frac{\left|\mathbb{B}_{\mathrm{kk}}\right|}{2 \cosh \left[\gamma\left(v \tilde{t}-\tilde{x}_{0}\right)\right]}\left(\mathrm{e}^{\gamma \tilde{x}}-\mathrm{e}^{-\gamma \tilde{x}}\right) \\
& =\mathrm{e}^{\gamma(\tilde{x}+b)}-\mathrm{e}^{-\gamma(\tilde{x}-b)}
\end{aligned}
$$

donde hemos escrito $\varphi=\phi_{\mathrm{kk}}=-\phi_{\overline{\mathrm{kk}}} \mathrm{y}$

$$
b(\tilde{t})=\frac{1}{\gamma} \ln \left\{\frac{1}{2}\left|\mathbb{B}_{\mathrm{kk}}\right| \operatorname{sech}\left[\gamma\left(v \tilde{t}-\tilde{x}_{0}\right)\right]\right\} .
$$

En las aproximaciones asintóticas para $|\tilde{t}| \gg 1$, es decir, tiempos lejanos; $b(\tilde{t})$ se comporta como

$$
\begin{aligned}
& b(\tilde{t}) \underset{\tilde{t} \rightarrow-\infty}{\longrightarrow}\left(v \tilde{t}-\tilde{x}_{0}\right)+\frac{1}{\gamma} \ln \left|\mathbb{B}_{\mathrm{kk}}\right| \\
& b(\tilde{t}) \underset{\tilde{t} \rightarrow+\infty}{\longrightarrow}-\left(v \tilde{t}-\tilde{x}_{0}\right)+\frac{1}{\gamma} \ln \left|\mathbb{B}_{\mathrm{kk}}\right|,
\end{aligned}
$$

escribiendo

$$
\Delta=\gamma^{-1} \ln \left|\mathbb{B}_{\mathrm{kk}}\right|=\gamma^{-1} \ln \left(\frac{v}{c}\right)
$$

la solución $\varphi$ tiene la forma

$$
\begin{aligned}
& \varphi_{\tilde{t} \rightarrow-\infty} \approx 4 \arctan \left[\mathrm{e}^{\gamma\left(\tilde{x}-\tilde{x}_{0}+v \tilde{t}+\Delta\right)}-\mathrm{e}^{-\gamma\left(\tilde{x}+\tilde{x}_{0}-v \tilde{t}-\Delta\right)}\right] \\
& \varphi_{\tilde{t} \rightarrow+\infty} \approx 4 \arctan \left[\mathrm{e}^{\gamma\left(\tilde{x}+\tilde{x}_{0}-v \tilde{t}+\Delta\right)}-\mathrm{e}^{-\gamma\left(\tilde{x}-\tilde{x}_{0}+v \tilde{t}-\Delta\right)}\right]
\end{aligned}
$$

si consideramos el solitón en $\tilde{x} \gg 0$, podemos escribir

$$
\varphi_{(\tilde{t}, \tilde{x}) \rightarrow(-\infty,+\infty)} \approx 4 \arctan \left[\mathrm{e}^{\gamma\left(\tilde{x}-\tilde{x}_{0}+v \tilde{t}+\Delta\right)}\right]=\phi_{\mathrm{k}}\left(\tilde{x}-\tilde{x}_{0}+v\left[\tilde{t}+\frac{\Delta}{v}\right]\right)
$$

mientras que en $\tilde{x} \ll 0$

$$
\varphi_{(\tilde{t}, \tilde{x}) \rightarrow(-\infty,-\infty)} \approx 4 \arctan \left[-\mathrm{e}^{-\gamma\left(\tilde{x}+\tilde{x}_{0}-v \tilde{t}-\Delta\right)}\right]=\phi_{\mathrm{k}}\left(\tilde{x}+\tilde{x}_{0}-v\left[\tilde{t}+\frac{\Delta}{v}\right]\right)
$$

por lo que (2.8.43a) representaba dos kinks

$$
\varphi_{\tilde{t} \rightarrow-\infty} \approx \phi_{\mathrm{k}}\left(\tilde{x}+v\left[\tilde{t}+\frac{\Delta}{v}\right]\right)+\phi_{\mathrm{k}}\left(\tilde{x}-v\left[\tilde{t}+\frac{\Delta}{v}\right]\right)
$$


mientras que en tiempos muy posteriores

$$
\begin{aligned}
\varphi_{\tilde{t} \rightarrow+\infty} & \approx 4 \arctan \left[\mathrm{e}^{\gamma\left(\tilde{x}+\tilde{x}_{0}-v \tilde{t}+\Delta\right)}\right]+4 \arctan \left[-\mathrm{e}^{-\gamma\left(\tilde{x}-\tilde{x}_{0}+v \tilde{t}-\Delta\right)}\right] \\
& \approx \phi_{\mathrm{k}}\left(\tilde{x}+\tilde{x}_{0}-v\left[\tilde{t}-\frac{\Delta}{v}\right]\right)+\phi_{\mathrm{k}}\left(\tilde{x}-\tilde{x}_{0}+u\left[\tilde{t}-\frac{\Delta}{v}\right]\right),
\end{aligned}
$$

por lo que (2.8.43b) representará dos kinks

$$
\varphi_{\tilde{t} \rightarrow+\infty} \approx \phi_{\mathrm{k}}\left(\tilde{x}-v\left[\tilde{t}-\frac{\Delta}{v}\right]\right)+\phi_{\mathrm{k}}\left(\tilde{x}+v\left[\tilde{t}-\frac{\Delta}{v}\right]\right) .
$$

El término $\Delta$ ó $\Delta / v$ representa un corrimiento o retraso, respectivamente; es consecuencia de los efectos elásticos de la colisión.

La solución antikink-antikink se obtiene al intercambiar el $\phi_{1} \leftrightarrow \phi_{2}$, es decir, el signo en las ecuaciones (2.8.37).

\subsubsection{Colisión kink-antikink}

Similarmente para generar esta solución podemos utilizar la formula (2.8.30), para a cual utilizamos

$$
\begin{aligned}
& \phi_{1}=4 \arctan \left\{\exp \left[\gamma_{1}\left(\tilde{x}-\tilde{x}_{01}-v_{1} \tilde{t}\right)\right]\right\}=\phi_{\mathrm{kink}}=\phi_{\mathrm{k}} \\
& \phi_{2}=4 \arctan \left\{\exp \left[\gamma_{2}\left(\tilde{x}-\tilde{x}_{02}-v_{2} \tilde{t}\right)\right]\right\}=\phi_{\mathrm{kink}}=\phi_{\mathrm{k}}
\end{aligned}
$$

dos kinks, $\phi_{1}$ que viaja de izquierda a derecha y $\phi_{2}$ en dirección opuesta, de derecha a izquierda ambos por simplicidad con la misma masa $m_{1}=m_{2}=m$, misma rapidez $v_{1}=-v_{2}=v>0$ y con posiciones iniciales simétricas $x_{0}=-x_{10}=x_{20}>0$, con estas condiciones

$$
\begin{aligned}
\zeta_{1} & =\gamma\left(\tilde{x}+\tilde{x}_{0}-v \tilde{t}\right) \\
\zeta_{2} & =\gamma\left(\tilde{x}-\tilde{x}_{0}+v \tilde{t}\right)
\end{aligned}
$$

y $\gamma=\left(1-\beta^{2}\right)^{-1 / 2}$, con $\beta=v / c$ en (2.8.34). Sustituyendo en (2.8.35) tenemos $\mathbb{B}_{\mathrm{k} \overline{\mathrm{k}}}=$ $-c / v=-\left|\mathbb{B}_{\mathrm{k} \overline{\mathrm{k}}}\right| \mathrm{y}$ la solución (2.8.34) es

$$
\phi_{\mathrm{k} \overline{\mathrm{k}}}=4 \arctan \left\{\frac{c \sinh \left[\gamma\left(v \tilde{t}-\tilde{x}_{o}\right)\right]}{v \cosh [\gamma \tilde{x}]}\right\}=-\phi_{\overline{\mathrm{kk}}}
$$

que en el tiempo representa la colisión de un kink y un antikink (Figura 2.8.5b). Similarmente a $(2.8 .39 \mathrm{~b})$, el comportamiento asintótico de $\phi_{\mathrm{k} \overline{\mathrm{k}}}$ lo analizamos reescribiendo

$$
\begin{aligned}
\tan \left(\frac{\psi}{4}\right) & =\left|\mathbb{B}_{\mathrm{k} \overline{\mathrm{k}}}\right| \sinh \left[\gamma\left(v \tilde{t}-\tilde{x}_{o}\right)\right] \operatorname{sech}(\gamma \tilde{x}) \\
& =\exp \ln \left|\mathbb{B}_{\mathrm{k} \overline{\mathrm{k}}}\right| 2 \sinh \left[\gamma\left(v \tilde{t}-\tilde{x}_{o}\right)\right] \frac{\operatorname{sech}(\gamma \tilde{x})}{2}
\end{aligned}
$$


donde escribimos $\psi=\phi_{\mathrm{k} \overline{\mathrm{k}}}=-\phi_{\overline{\mathrm{kk}}}$ considerando las aproximaciones asintóticas temporales como

$$
\begin{array}{lll}
2 \sinh \left[\gamma\left(v \tilde{t}-\tilde{x}_{o}\right)\right] & \underset{\tilde{t} \rightarrow-\infty}{\longrightarrow}-\mathrm{e}^{-\gamma\left(v \tilde{t}-\tilde{x}_{0}\right)} \\
2 \sinh \left[\gamma\left(v \tilde{t}-\tilde{x}_{o}\right)\right] & \underset{\tilde{t} \rightarrow+\infty}{\longrightarrow} & \mathrm{e}^{\gamma\left(v \tilde{t}-\tilde{x}_{0}\right)}
\end{array}
$$

la solución $\psi$ puede escribirse con $\Delta=\gamma^{-1} \ln \left|\mathbb{B}_{\mathrm{k} \overline{\mathrm{k}}}\right|$

$$
\begin{aligned}
& \psi_{\tilde{t} \rightarrow-\infty} \approx 4 \arctan \left[\frac{-\mathrm{e}^{\gamma \Delta} \mathrm{e}^{-\gamma\left(v \tilde{t}-\tilde{x}_{0}\right)}}{\mathrm{e}^{\gamma \tilde{x}}+\mathrm{e}^{-\gamma \tilde{x}}}\right] \\
& \psi_{\tilde{t} \rightarrow+\infty} \approx 4 \arctan \left[\frac{\mathrm{e}^{\gamma \Delta} \mathrm{e}^{\gamma\left(v \tilde{t}-\tilde{x}_{0}\right)}}{\mathrm{e}^{\gamma \tilde{x}}+\mathrm{e}^{-\gamma \tilde{x}}}\right]
\end{aligned}
$$

si consideramos el solitón en $\tilde{x} \gg 0$, podemos escribir

$$
\psi_{(\tilde{t}, \tilde{x}) \rightarrow(-\infty,+\infty)} \approx 4 \arctan \left[-\mathrm{e}^{-\gamma\left(\tilde{x}-\tilde{x}_{0}+v \tilde{t}-\Delta\right)}\right]=\phi_{\mathrm{k}}\left(\tilde{x}+v\left[\tilde{t}-\frac{\Delta}{v}\right]\right)
$$

mientras que en $\tilde{x} \ll 0$

$$
\psi_{(\tilde{t}, \tilde{x}) \rightarrow(-\infty,-\infty)} \approx 4 \arctan \left[-\mathrm{e}^{\gamma\left(\tilde{x}+\tilde{x}_{0}-v \tilde{t}+\Delta\right)}\right]=\phi_{\overline{\mathrm{k}}}\left(\tilde{x}+\tilde{x}_{0}-v\left[\tilde{t}-\frac{\Delta}{v}\right]\right)
$$

por lo que (2.8.54a) representaba un kink y un antikink

$$
\psi_{\tilde{t} \rightarrow-\infty} \approx \phi_{\mathrm{k}}\left(\tilde{x}+v\left[\tilde{t}+\frac{\Delta}{v}\right]\right)+\phi_{\overline{\mathrm{k}}}\left(\tilde{x}-v\left[\tilde{t}+\frac{\Delta}{v}\right]\right),
$$

mientras que en tiempos muy posteriores

$$
\begin{aligned}
\psi_{\tilde{t} \rightarrow+\infty} & \approx 4 \arctan \left[\mathrm{e}^{-\gamma\left(\tilde{x}+\tilde{x}_{0}-v \tilde{t}-\Delta\right)}\right]+4 \arctan \left[\mathrm{e}^{\gamma\left(\tilde{x}-\tilde{x}_{0}+v \tilde{t}+\Delta\right)}\right] \\
& \approx \phi_{\overline{\mathrm{k}}}\left(\tilde{x}+\tilde{x}_{0}-v\left[\tilde{t}+\frac{\Delta}{v}\right]\right)+\phi_{\mathrm{k}}\left(\tilde{x}-\tilde{x}_{0}+v\left[\tilde{t}+\frac{\Delta}{v}\right]\right),
\end{aligned}
$$

con lo que (2.8.54b) representará un antikink y un kink

$$
\psi_{\tilde{t} \rightarrow+\infty} \approx \phi_{\overline{\mathrm{k}}}\left(\tilde{x}-v\left[\tilde{t}-\frac{\Delta}{v}\right]\right)+\phi_{\mathrm{k}}\left(\tilde{x}+v\left[\tilde{t}-\frac{\Delta}{v}\right]\right) .
$$

Al igual que en la colisión kink-kink (antikink-antikink) El término $\Delta$ ó $\Delta / v$ representa un corrimiento o retraso, respectivamente, y es consecuencia de los efectos elásticos de la colisión.

Las soluciones anteriores reflejan dos solitones en un pasado lejano y dos solitones en un futuro distante a la colisión. Antes de la colisión ambos solitones se encuentran ya sea en un mismo sector topológico o en dos adyacentes, durante la colisión parecen distorsionarse mientras se acercan, y después de la colisión recuperan su forma y velocidad con un posible retraso en el tiempo. 


\subsubsection{Solución tipo breather}

El tercer tipo de solución mencionado, el breather corresponde a un kink seguido de un antikink que viajan en el mismo sentido con la misma velocidad sin variar la distancia entre ellos; es decir, dos solitones continuos que en un pasado lejano, y que en un futuro distante tienen una forma y velocidad definida. En este caso no hay retraso, ya que no hay colisión. Esta solución es un caso especial de una solución más general encontrada por Perring y Skyrme (1962); quienes utilizaron el modelo se sine-Gordon para explicar unas partículas hipotéticas conocidas como skyrmiones utilizando solitones topológicos.

Para obtener la solución tipo breather, partimos de la solución kink-antikink. Permitamos que la velocidad $v$ tome un valor imaginario en la ecuación (2.8.51), por ejemplo, $v=i \omega$ con $\omega<1$, además $x_{0}=0$. En este punto $\omega$ es un parámetro, no una velocidad, obtenemos así

$$
\begin{aligned}
\phi_{\mathrm{b}} & =4 \arctan \left[\frac{c \sinh (\gamma v \tilde{t})}{v \cosh (\gamma \tilde{x})}\right] \\
& =4 \arctan \left[\frac{c \sin (\gamma \omega \tilde{t})}{\omega \cosh (\gamma \tilde{x})}\right]
\end{aligned}
$$

donde $\gamma=c\left(c^{2}+\omega^{2}\right)^{-1 / 2}$. En esta solución al modelo sine-Gordon, la función $\phi_{\mathrm{k} \overline{\mathrm{k}}}$ viaja asintóticamente a velocidad $v$ y el breather se puede interpretar con un estado ligado del par kink-antikink. Esta solución es periódica y oscila con el período

$$
\tau=\frac{2 \pi \sqrt{c^{2}+\omega^{2}}}{c \omega}
$$

dicha oscilación es del kink respecto al antikink.

Mientras que en la solución $\phi_{\mathrm{k} \overline{\mathrm{k}}}$ los solitones se separan con el avance (retroceso) del tiempo, aquí sólo se separan una distancia finita y nunca se liberan completamente de la distorsión por lo que oscilan uno respecto del otro. En este caso también se presenta cualitativamente el retraso $\Delta$ de $\phi_{\mathrm{k} \overline{\mathrm{k}}}$, y se puede interpretar como resultado de la fuerza de atracción que sufren ambos solitones; Figura 2.8.6. 


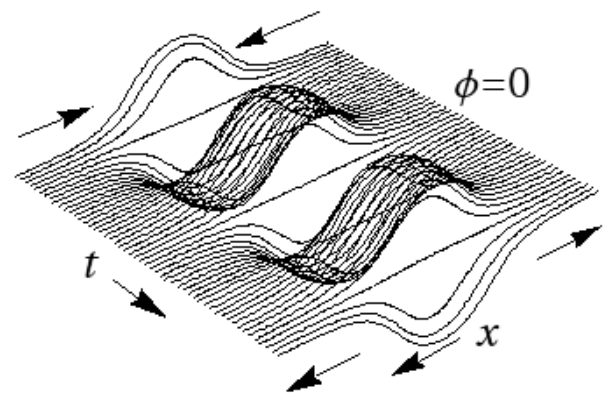

Figura 2.8.6: Solitón breather. 



\section{Capítulo 3}

\section{Halos de materia oscura}

El comportamiento de objetos galácticos, es de gran relevancia para describir la evolución

del universo. Sin embargo, en la actual concepción la materia oscura junto con la energía oscura, son al parecer los principales componentes de éste, y continúan siendo de las principales incógnitas de la física. Por lo cual para comprender el comportamiento y evolución de objetos en el universo es necesario comprender el comportamiento de la materia oscura.

Debido a que los efectos de la materia oscura son principalmente gravitacionales, los sistemas que presentan estas manifestaciones son de escala galáctica o mayor. Dichos sistemas cuyo comportamiento no se explica solamente con la materia visible, suelen al parecer formar halos alrededor de objetos astrofísicos. Los llamados halos de materia oscura, se considera concentran la mayor cantidad de la masa del objeto que encierran, en el caso de las galaxias son al parecer responsables de las curvas de rotación observadas en las estrellas. Desde un punto de vista de cosmológico están relacionados con la formación y movimiento de galaxias y en el comportamiento de cúmulos de galaxias.

\subsection{Materia oscura}

La propuesta realizada por Fritz Zwicky $(1933,2008)$ de la materia oscura, se dio como una respuesta a la masa faltante que amarra gravitacionalmente el cúmulo Coma; esto debido a que, según las observaciones, la masa luminosa no era suficiente. Posteriormente a inicios de los 70's Vera Rubin por medio de observaciones extendió estas ideas a las galaxias, observando que las curvas de rotación de las observaciones requerían después de cierto radio más masa que la luminosa; hecho que sugería la existencia de un halo oscuro alrededor de galaxias en espiral.

Posteriormente Persic et al. (1996) mostraron que la estructura de galaxias en espiral conectaba la materia luminosa y la oscura, con las curvas de rotación. En galaxias con 
poca luminosidad el halo de materia oscura inferido tiene un efecto dominante, mientras que con galaxias altamente luminosas el efecto es contrario. Por lo anterior, podemos decir que una de las principales manifestaciones de la existencia de materia oscura son los halos alrededor de objetos astrofísicos.

En modelos actuales, la materia oscura se encuentra compuesta de partículas que interactúan muy débilmente, partículas que únicamente se mueven bajo la influencia de la gravedad y que presumiblemente no colisionan entre sí. Estos modelos proponen candidatos para estas partículas, entre las cuales se incluyen objetos bariónicos como los llamados halos masivos compactos (MACHOs, Massive Compact Halo Object) y estrellas de bosones (BS, Boson Stars). También, objetos no bariónicos formados de partículas masivas débilmente interactuantes (WIMPs, Weakly Interacting Massive Particles), o de axiones. Sin embargo, los objetos bariónicos parecen ser insuficientes para los efectos observados, mientras que los no bariónicos no se han detectado experimental u observacionalmente de manera clara.

De los candidatos mencionados, WIMPs y axiones se creía que eran indistinguibles a nivel observacional, pero se ha mostrado en trabajos recientes, como los realizados por Sikivie y Yang (2009); Erken et al. (2011), que sí podrían distinguirse.

\subsubsection{Modelos de materia oscura}

Las observaciones en la cosmología moderna establecen que aproximadamente el $26.8 \%$ de la composición del universo se considera materia oscura. El gran porcentaje de materia oscura hace necesario un modelo que describa su fenomenología en su totalidad. Para esto, se han utilizado muchos modelos entre los cuales materia oscura fría (CDM, Cold Dark Matter) es quizá el más exitoso; Figura 3.1.1.

Si bien a nivel cosmológico, el modelo de materia oscura fría con constante cosmológica ( $\Lambda$ CDM, Lambda Cold Dark Matter), ha mostrado en simulaciones numéricas ser un modelo funcional en la formación de estructuras más grandes que galaxias, en escalas galáctica o subgaláctica la teoría presenta problemas y los resultados no son del todo adecuados. Esto ha llevado a que se propongan otros modelos alternativos, como el de campo escalar para materia oscura (SFDM, Scalar Field Dark Matter), primero postulado por Sin (1994) y Schunck (1998). A la par, se han propuesto modelos que no utilizan materia oscura y en su lugar modifican la ley de gravitación, entre estos modelo se encuentra la dinámica Newtoniana modificada (MOND, Modified Newtonian Dynamics), gravedad tensorial-vectorial-escalar (TeVeS, Tensor-Vector-Scalar gravity), gravedad escalar-tensorial-vectorial (STVG, Scalar-Tensor-Vector Gravity), gravedad no simétrica (NGT, Non-symmetric Gravitational Theory), etc. 


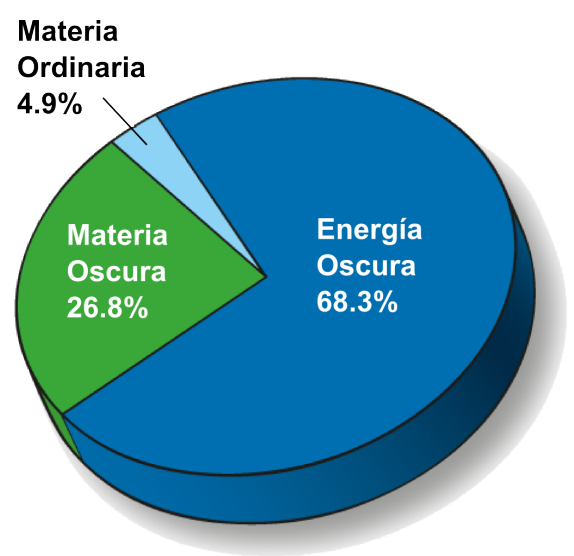

Figura 3.1.1: Componentes del Universo, la más abundante la energía oscura $68.3 \%$ (desconocida), la materia oscura casi el $26.8 \%$ (desconocida) y el restante $4.9 \%$ que incluye materia luminosa y no luminosa (conocida parcialmente) esta incluye estrellas, átomos, gas intergaláctico, radiación, neutrinos y otros.

\subsubsection{Materia oscura fría con constante cosmológica: $\Lambda \mathrm{CDM}$}

El modelo $\Lambda$ CDM tiene tres principales discrepancias entre predicción y observación. Primeramente el modelo predice que la distribución de densidad de los halos de materia oscura debe tener una cúspide o pico en el centro, diferente a lo observado en las curvas de rotación (cuspy halo problem); también predice la formación de un gran número de galaxias satélites, de las cuales sólo se observan muy pocas (missing satellite problem), así como significantes cantidades de materia de poco momento angular la cual esta ausente en las galaxias.

$\Lambda \mathrm{CDM}$ es un modelo en el cual la materia no puede observarse por su radiación electromagnética y cuyas partículas se mueven lentamente comparado con la velocidad de la luz. En este modelo las estructuras se forman a partir de la unión de estructuras menores para formar estructuras de mayor masa, un esquema llamado bottom-up; que implica una escala mínima natural para materia oscura. Sin embargo, el modelo no predice el tipo de partículas que intervienen, lo que permite al menos tres categorías de posibles candidatos: MACHOs, WIMPs y axiones.

\subsubsection{Materia oscura escalar: SFDM}

Para solventar los problemas que presenta $\Lambda$ CDM, se han propuesto algunos modelos. Como el de Spergel y Steinhardt (2000) (cf. Riotto y Tkachev (2000)) que proponen materia oscura fría con interacción, otros consideran la materia oscura como un campo escalar (SF, Scalar Field); también modelos de condensado de Bose-Einstein (BEC, BoseEinstein Condensate) o modelos de estrellas de bosones ambos para halos de materia 


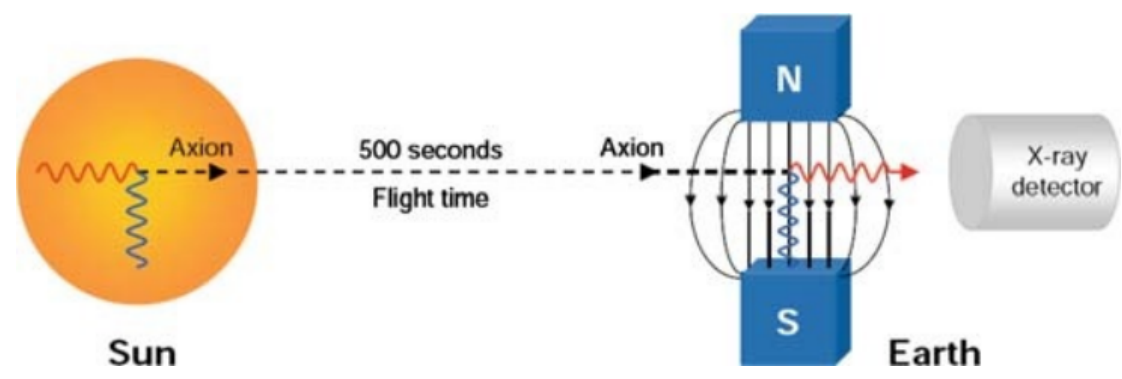

Figura 3.1.2: Principio del funcionamiento de un helioscopio de axiones. Los axiones producidos en el núcleo del Sol por efecto Primakoff, serían convertidos nuevamente en fotones en un fuerte campo magnético. Posteriormente estos fotones con el mismo espectro de energía que los axiones serían recogidos por el detector de rayos-X; Kuster et al. (2008), Cap. 10; Vogel et al. (2015).

oscura. Algunos han mostrado ser exitosos tanto a escala cosmológica como a escala galáctica o subgaláctica, además de ofrecer algunas ventajas sobre $\Lambda$ CDM. Sin embargo, aún no se ha estudiado toda la fenomenología que ha sido estudiada con $\Lambda$ CDM.

Estos modelos, hasta ahora se consideran de juguete, ya que campos escalares, axiones y neutralinos, todavía no se han detectado en los aceleradores de partículas o en los helioscopios de axiones, Figura 3.1.2; vid. Zioutas et al. (2004); Kuster et al. (2008), Cap. 10. Estos modelos basados en SFDM o BEC se basan en partículas ultra ligeras y explican bien la suavidad de las curvas de rotación observadas en galaxias, pueden estar libres del problema de cúspide de las curvas de rotación y del problema de galaxias satélites faltantes; resolviendo los problemas de $\Lambda$ CDM. Otro mérito del modelo es que las colisiones de objetos astrofísicos se pueden describir como colisiones de estrella de bosones; vid. Lee (2009).

Trabajos recientes de Sikivie y Yang (2009); Erken et al. (2011) han mostrado que los axiones pueden termalizar, es decir, alcanzar un equilibrio térmico, lo que les permitiría formar un condensado de Bose-Einstein, que puede representarse por un campo escalar y permite utilizar dicha conjetura en el modelo.

\subsection{Propiedades de la materia oscura}

Se ha observado o conjeturado que la materia oscura debe poseer al menos cinco características básicas:

Ópticamente oscura La materia oscura no brilla, es decir, no debe interactuar o interactúa muy débilmente con la radiación electromagnética. La principal consecuencia de esto es que este tipo de materia no debe enfriarse por radiación de fotones, por lo cual no colapsa al centro de las galaxias, como lo hace la materia bariónica. 
No colisionar Esta propiedad constriñe a que la materia oscura debe autointeraccionar, algo importante a altas densidades y distancias cortas. A la vez esto podría servir para borrar las estructura a pequeña escala en los halos galácticos y borrar los picos de densidad esperados en los núcleos de galaxias.

Fría A principios de 1980, se observo que si la materia oscura era fría se podían explicar las propiedades de las galaxias. La materia oscura debe ser suficientemente no-relativista, es decir, la velocidad de dispersión de las partículas es suficientemente pequeña $\left(\sim 10^{-8} c\right)$, para que en la época de igualdad de materia y radiación, permita la existencia de estructuras pequeñas. De tal manera que la velocidad puede considerarse cero para grandes estructuras y halos galácticos.

Fluido Esta materia debe ser suficientemente fina en escalas de galaxias, tal que su granularidad no sea detectable. De no ser así, se presentaría una dependencia temporal en el campo gravitacional, además de inducir un ruido de Poisson en el espectro de fluctuaciones de densidad.

Clásica Debe ser suficientemente clásica para estar confinada en escalas de galaxias. Por ejemplo, si la materia oscura estuviese formada de bosones, ésta debería confinarse en escalas de kiloparsecs, esto también corrige los picos de densidad en los núcleos galácticos. De no ser así, su naturaleza cuántica sólo se manifestaría si su masa fuese excesivamente pequeña.

De estas cinco propiedades las primeras tres restringen el espacio de posibles candidatos, mientras que las dos últimas establecen cierto límite respecto a la masa de las partículas; vid. Baltz (2004)

\subsection{Halos de materia oscura}

Los halos de materia oscura se forman a partir del colapso gravitacional de materia, este colapso es un proceso no lineal, el cual borra el proceso de formación del halo, esto permite considerar únicamente su forma final. Esta forma puede aproximarse por superficies descritas por un perfil de densidad únicamente dependiente del radio, bajo dicha condición la forma que toman puede considerarse esférica o en casos más generales elipsoidales; aunque en estos últimos pueden aparecer centros de masa en distintos lugares. La forma de los halos se ha determinado por diferentes técnicas: usando las órbitas de galaxias satélites trazando sus fuerzas de marea; a partir de la forma de los halos, de los rayos-X alrededor de galaxias y cúmulos vía lentes gravitacionales; y usando la distribución angular de las galaxias satélites. Cada una de estas técnicas con pros y contras; vid. Mo et al. (2010). 
Un interesante comportamiento de estos halos se presenta en la colisión de cúmulos de galaxias, ya que en dichas colisiones el comportamiento observado no es compatible con el esperado únicamente por la materia visible y el gas caliente; por lo que se considera que la presencia de materia oscura tiene un comportamiento dominante. En particular, cúmulos como el Bullet (1E 0657-56), requieren la ayuda de materia oscura para explicar el comportamiento observado. Otra opción, es una supuesta modificación de gravedad, en general no aceptada; vid. Brownstein y Moffat (2007); Clowe et al. (2007).

Durante las colisiones de cúmulos de galaxias se pueden distinguir tres elementos que tienen diferente comportamiento: las galaxias compuestas de estrellas, el gas caliente entre galaxias (principalmente hidrógeno) y materia oscura. En la colisión del cúmulo se considera que las estrellas, debido a su separación, no colisionan. Así, en una colisión se espera que tanto la materia oscura y las estrellas se mueven juntas, inclusive en una colisión violenta. Mientras que el gas caliente sí auto-interacciona electromagnéticamente y deja la demás materia al centro de la colisión.

La débil interacción de la materia oscura permite especular que aún en colisiones violentas la materia oscura puede comportarse como un objeto casi invariante, un objeto que puede sobrevivir a una colisión, un proceso que puede modelarse como un solitón. Para hacer esto, la distribución de materia oscura se puede inferir por efectos de lentes gravitacionales, mientras que el gas y estrellas por rayos-X. Esto permite especular sobre el comportamiento de algunos cúmulos, por ejemplo el Bullet y el Abell 520. En estos ejemplos, la característica de que no colisionan las partículas de materia oscura parece contradictoria. Mientras que en el Bullet se cumple (Figura 3.3.1), en el Abell 520 no; en este último las estrellas dejan a la materia oscura y el gas en el centro de la colisión. Esta contradicción es difícil de explicar en el contexto de $\Lambda$ CDM y MOND, sin embargo; en modelos solitónicos podría explicarse debido a la estabilidad de los solitones considerados. Recientes observaciones sobre los cúmulos Abell 520 y Bullet parecen profundizar el misterio; vid. Mahdavi et al. (2007).

Otra justificación de modelos de este tipo, se da para la colisión de dos cúmulos de galaxias, ya que si la energía cinética es suficientemente grande se puede presentar un comportamiento tipo solitón (una interacción elástica); tal como aparentemente se observa en el cúmulo Bullet. Si la energía no es suficiente los halos se mezclan debido a la poca interacción repulsiva (una interacción inelástica) entre la materia oscura, tal como aparentemente sucede en el cúmulo Abell 520. Ideas como esta podrían explicar el origen de galaxias oscuras (Dark galaxies) y de galaxias sin materia oscura (White galaxies); vid. Lee et al. (2008). 

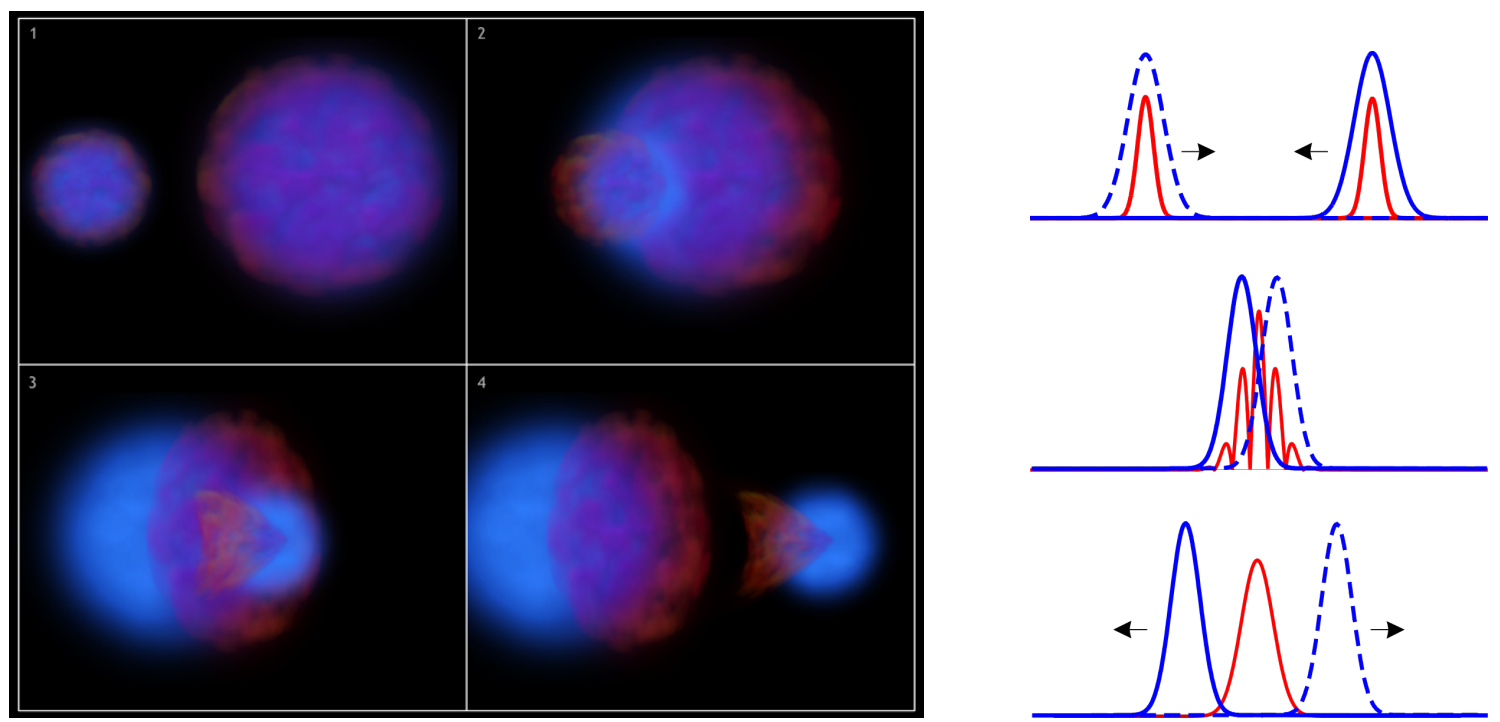

Figura 3.3.1: Vista artística del comportamiento que se presenta en el cúmulo Bullet; NA$\mathrm{SA} / \mathrm{CXC}$ M. Weiss. Representación en ondas del comportamiento.

\subsection{Axión: Un candidato de materia oscura}

El Lagrangiano $L_{\mathrm{SM}}$ en cromodinámica cuántica (QCD, quantum chromodynamics) es estándar y basado en las teorías de norma no-abelianas, a pesar de esto, presenta problemas. Uno de estos se dio con el descubrimiento del las soluciones tipo instantón topológicamente clasificadas por un término Pontrjagin del tipo $\vec{E} \cdot \vec{B}$. En el Lagrangiano de QCD para $N$ sabores, cuando las masas de los quarks se anulan se tienen una simetría global $U(N)_{V} \times U(N)_{A}$, simetrías tanto vectorial como axial. Pero, al considerar estas simetrías se observa que la estructura de vacío QCD tiene una estructura más compleja, que resulta en que $U(1)_{A}$ no es una simetría real de QCD, aunque es una simetría aparente del Lagrangiano cuando las masas de los quarks tienden al limite cero. Sin embargo, con este vacío más complicado hay un parámetro de fase $\theta$ asociado, el cual sólo si es muy pequeño no rompe la simetría $C P$. Experimentalmente los datos del momento dipolar del neutrón avalan un valor muy pequeño; vid. Sozzi (2008).

A este problema existen algunas posibles soluciones; sin embargo, la propuesta por Robert Peccei y Helen Quinn (1977) resultó más atractiva. Dicha solución se basa en la simetría introducida originalmente por Steven Weinberg (1975), y soluciona el problema de violación $C P$ fuerte (strong $C P$ problem) en $U(1)_{A}$, postulando una nueva simetría $U(1)$, posteriormente denominada $U(1)_{\mathrm{PQ}}$ en el modelo estándar. La simetría de Peccei y Quinn (1977) $U(1)_{\mathrm{PQ}}$, introduce un campo dinámico, y asocia una partícula ligera y de débil interacción, como un pseudobosón tipo Nambu-Goldstone asociado al rompimiento de la nueva simetría. Esta partícula fue nombrada por Frank Wilczek como axión, ya que 
"limpia" el problema con una corriente axial; vid. Kuster et al. (2008).

Posteriormente Weinberg (1978) y Wilczek (1978) lo consideraron un candidato para materia oscura fría. Y actualmente forma parte de la lista de los candidatos más plausibles para materia oscura, junto con las partículas supersimétricas más ligeras (LSP, lightest supersimetric particles); como el axino y gravitino; los supercompañeros fermiónicos del axión y gravitón respectivamente. Algo claro hasta ahora es que nada indica que sólo exista un tipo de materia oscura; modelos como los estudiados por Marco Baldi (2012), consideran distintos tipos de materia oscura acoplada a la energía oscura.

\subsubsection{Lagrangiano del axión}

El axión predicho en el mecanismo de Peccei y Quinn (1977) es el resultado del rompimiento espontáneo de la simetría $U(1)_{\mathrm{PQ}}$ a cierta escala $f_{a}$. La resolución del vacío de QCD añade un término extra al Lagrangiano de QCD, esto lleva a un Lagrangiano efectivo de la forma

$$
L_{\mathrm{eff}}=L_{\mathrm{QCD}}+\frac{1}{2} \partial_{\mu} a \partial^{\mu} a+\xi \frac{g_{s}^{2}}{32 \pi^{2}} \frac{a}{f_{a}} G_{b \mu \nu} \tilde{G}_{b}^{\mu \nu} .
$$

En éste, el último término viola las simetrías de paridad $P$ y inversión temporal $T$, pero conserva la conjugación carga $C$, por lo cual viola $C P$. El término mencionado representa la interacción axión-gluon, que a la vez es la interacción con dos fotones, y el acoplamiento de los campos eléctrico y magnético al campo del axión, un término de Pontryagin,

$$
L_{a \gamma \gamma}=\frac{g_{s}^{2}}{32 \pi^{2}} \frac{a}{f_{a}} G_{b \mu \nu} \tilde{G}_{b}^{\mu \nu}=\frac{g_{a \gamma \gamma}}{4} F_{\mu \nu} \tilde{F}^{\mu \nu} a=-g_{a \gamma \gamma} \vec{E} \cdot \vec{B} a
$$

El término es necesario para asegurar que la corriente de $U(1)_{\mathrm{PQ}}$ tenga la anomalía quiral, importante para compensar la violación de simetría

$$
\partial_{\mu} J_{\mathrm{PQ}}^{\mu}=\xi \frac{g_{s}^{2}}{32 \pi^{2}} G_{b \mu \nu} \tilde{G}_{b}^{\mu \nu}
$$

Dicho término también representa un potencial efectivo cuyo mínimo

$$
\left\langle\frac{\partial V_{\mathrm{eff}}}{\partial a}\right\rangle=-\left.\frac{\xi}{f_{a}} \frac{g_{s}^{2}}{32 \pi^{2}}\left\langle G_{b}^{\mu \nu} \tilde{G}_{b \mu \nu}\right\rangle\right|_{\langle a\rangle}=0
$$

ocurre en $\langle a\rangle=-\bar{\theta} f_{a} / \xi$, esto a la vez provee una solución dinámica al problema $C P$.

Incluyendo los efectos de la anomalía de QCD se genera un potencial efectivo $V_{\text {eff }}$, el potencial obtenido es periódico para el campo del axión en el ángulo efectivo de vacío $\bar{\theta}+\langle a\rangle \xi / f_{a}$ es

$$
V_{\mathrm{eff}} \sim \cos \left(\bar{\theta}+\langle a\rangle \frac{\xi}{f_{a}}\right)
$$


y expandiendo (3.4.5) alrededor del mínimo $\langle a\rangle$ se puede obtener la masa del axión

$$
m_{a}^{2}=\left\langle\frac{\partial^{2} V_{\mathrm{eff}}}{\partial a^{2}}\right\rangle=-\left.\frac{\xi}{f_{a}} \frac{g_{s}^{2}}{32 \pi^{2}} \frac{\partial}{\partial a}\left\langle G_{b}^{\mu \nu} \tilde{G}_{b \mu \nu}\right\rangle\right|_{\langle a\rangle=-\bar{\theta} f_{a} / \xi} .
$$

El parámetro clave para definir las características del axión es la escala del rompimiento de simetría $f_{a}$.

\subsubsection{Potencial efectivo del axión}

Para escribir un potencial del axión más preciso, es necesario considerar los efectos no perturbativos de QCD asociados con instantones, y considerar que estos colapsan el rompimiento de la simetría $U(1)_{\mathrm{PQ}}$ al subgrupo discreto $Z(N)$. Como consecuencia el potencial es periódico con periodo $\Delta a=2 \pi v_{a} / N=2 \pi f_{a}$. Finalmente el potencial efectivo se puede escribir como

$$
\begin{aligned}
V_{\mathrm{eff}}(\phi) & =f_{a}^{2} m_{a}^{2}\left[1-\cos \left(\phi / f_{a}\right)\right] \\
& \simeq m_{a}^{2}\left[\frac{1}{2} \phi^{2}-\frac{1}{4 ! f_{a}^{2}} \phi^{4}+\frac{1}{6 ! f_{a}^{4}} \phi^{6}-\cdots\right]
\end{aligned}
$$

donde $m_{a}$ es la masa del axión. La curva resultante del potencial con el mínimo localizado en el origen puede ser interpretada como la masa del axión inducida. Masa que para una temperatura cero es del orden de $6 \mu \mathrm{eV}$; vid. Kuster et al. (2008).

El potencial (3.4.7) puede escribirse en una forma más usual como

$$
V_{\mathrm{eff}}(\phi)=\frac{m^{4}}{\lambda}\left[1-\cos \left(\frac{\sqrt{\lambda}}{m} \phi\right)\right],
$$

e introduciéndolo en la ecuación de onda (2.4.1) obtenemos

$$
\square \phi-\frac{m^{3}}{\sqrt{\lambda}} \sin \left(\frac{\sqrt{\lambda}}{m} \phi\right)=0,
$$

que es la conocida ecuación sine-Gordon.

\subsubsection{Axión como materia oscura}

Considerando las propiedades observadas para la materia oscura. Se puede mencionar que trabajos como los de Sikivie y Yang (2009); Erken et al. (2011); Sikivie (2012) han sugerido que los axiones pueden comportarse como un condensado de Bose-Einstein, lo que puede tener consecuencias observacionales respecto a la una posible distinción de varios tipos de materia oscura.

Si bien, a nivel fundamental, los axiones son descritos por campos cuánticos, estos pueden ser muy bien descritos en los límites semi-clásicos de dichos campos. En este límite, 


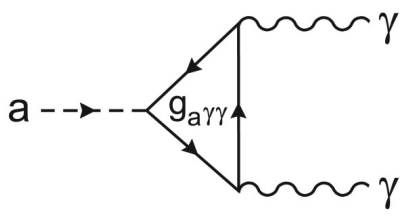

Figura 3.4.1: Diagrama de Feynman, que representa el acoplamiento no nulo de los fotones y el axión, representa como un axión puede convertirse en fotón y viceversa.

la energía $E=\mathcal{N} \hbar \omega$ se mantiene fija, así como el número de ocupación. Sin embargo, si los axiones termalizan, estos no se describen por un campo clásico, en su lugar se forma un condensado de Bose-Einstein.

Una de la fuentes de axiones fríos, explicitada por la cosmología considerando la transición de fase en QCD durante el universo temprano, es cuando los axiones son producidos al activar la masa del axión $m_{a}$ durante la transición. A órdenes de temperatura $T_{1} \simeq 1 \mathrm{GeV}$ $\left(\approx 10^{15} \mathrm{~K}\right)$, donde el tiempo crítico $t_{1} \simeq 20 \mu \mathrm{s}$ es definido a partir de la masa $m_{a}\left(t_{1}\right) t_{1}=1$, con lo que la masa del axión a temperatura cero en términos de $f_{a}$ se puede aproximar por

$$
m_{a} \simeq 6 \times 10^{-6} \mathrm{eV}\left(\frac{10^{12} \mathrm{GeV}}{f_{a}}\right),
$$

con $f_{a}$ como la constante de decaimiento del axión. De esta constante dependen primordialmente las propiedades de la partícula, aunque su valor no es conocido. Si bien, tanto la teoría de partículas como la cosmología permiten limitar el valor de este parámetro a

$$
3 \times 10^{9} \mathrm{GeV} \lesssim f_{a} \lesssim 10^{12} \mathrm{GeV}
$$

El parámetro clave para definir las características del axión es la escala de rompimiento espontáneo de simetría $U(1)_{\mathrm{PQ}}$ o constante de decaimiento $f_{a}$, y en términos de la cual se puede definir tanto la masa (3.4.11) como el acoplamiento de axiones a fotones

$$
g_{a \gamma \gamma} \equiv \frac{\alpha}{2 \pi f_{a}} C
$$

donde $\alpha$ es la constante de estructura fina y $C$ es un parámetro de acoplamiento del modelo. Para los axiones denotados por KSVZ (Kim-Shifman-Vainshtein-Zakharov) $C \sim 0.97, \mathrm{y}$ para los denotados por DFSZ (Dine-Fischler-Srednicki-Zhitnitshii) -0.36; Figura 3.4.1; vid. Kuster et al. (2008), Cap. 8.

Frank Wilczek y colaboradores plantean que en escenarios antrópicos la constante de acoplamiento $f_{a}$ es mayor que $10^{13} \mathrm{GeV}$; vid. Kim (1979); Preskill et al. (1983); Hertzberg et al. (2008); Kim y Carosi (2010). 


\subsubsection{Condensación de axiones}

Para la formación de un condensado de Bose-Einstein se pueden considerar cuatro condiciones básicas:

1. las partículas son bosones idénticos y están altamente condensadas en el espacio fase,

2. el número de partículas se conserva,

3. las partículas están en equilibrio térmico y abajo de la temperatura crítica.

Para el caso de los axiones las primeras dos condiciones se cumplen sin gran complicación, la tercera no es manifiestamente satisfecha. Sin embargo, en trabajos recientes Sikivie y Yang (2009); Erken et al. (2012) se muestra que tanto para auto-interacciones gravitacionales, como del tipo $\phi^{4}$, la termalización es posible, ya que la razón de relajación en términos del numeró de ocupación es

$$
\Gamma \sim \frac{\dot{\mathcal{N}}}{\mathcal{N}}
$$

la cual depende de la velocidad de dispersión $\delta v$ y de la sección eficaz $\sigma$.

Para esto Sikivie y Yang (2009) aproximan la densidad de número a un valor de

$$
n(t) \sim \frac{4 \times 10^{47}}{\mathrm{~cm}^{3}}\left(\frac{f_{a}}{10^{12} \mathrm{GeV}}\right)^{5 / 3}\left(\frac{a\left(t_{1}\right)}{a(t)}\right)^{3}
$$

donde $a(t)$ es el factor de expansión en el modelo de Friedmann, resultando en estos términos que la velocidad de dispersión es

$$
\delta v(t) \sim \frac{1}{m_{a} t_{1}} \frac{a\left(t_{1}\right)}{a(t)},
$$

lo que se da si la interacción entre los axiones es despreciable, caso referido como el límite de axiones fríos desacoplados. Con esto, el número de ocupación promedio por estado es

$$
\mathcal{N} \sim n \frac{(2 \pi)^{3}}{\frac{4 \pi}{3}(m \delta v)^{3}} \sim 10^{61}\left(\frac{f_{a}}{10^{12} \mathrm{GeV}}\right)^{8 / 3},
$$

este valor es mucho mayor que uno, lo que nos dice que la temperatura efectiva de axiones fríos es mucho más pequeña que la temperatura crítica para la condensación es en este caso

$$
T_{c}=\left(\frac{\pi^{2} n}{\zeta(3)}\right)^{1 / 3} \simeq 300 \mathrm{GeV}\left(\frac{f_{a}}{10^{12} \mathrm{GeV}}\right)^{5 / 9} \frac{a\left(t_{1}\right)}{a(t)} .
$$

Sikivie et al. también plantean las condiciones que llevan a que la temperatura para la formación del BEC es

$$
T_{\mathrm{BEC}} \sim 500 \mathrm{eV}\left(\frac{f_{a}}{10^{12} \mathrm{GeV}}\right)^{1 / 2}
$$


Una consideración más que debe hacerse es que en el límite $T=0$, en lugar de considerar la longitud de onda de De Broglie $\lambda_{\text {de Broglie }}=\left(2 \pi \hbar^{2} /(m k T)\right)^{1 / 2}$, debe considerarse la longitud de onda de Compton. Esto conlleva a que para que el BEC pueda considerarse como un halo de materia oscura, la longitud de onda de Compton de las partículas de materia oscura deben ser del orden del tamaño de la galaxia, es decir,

$$
\lambda_{\text {Compton }}=\frac{h}{m c} \sim 10 \mathrm{kpc} .
$$

Esto lleva a una aproximación en la masa de $m_{a} \simeq 10^{-26} \mathrm{eV}$, que es veinte órdenes de magnitud mayor que la masa usual (3.4.11), y está relacionada con los escenarios antrópicos planteados por Wilczek. Esta extrapolación requiere que la densidad de número sea pequeña; vid. Preskill et al. (1983); Hertzberg et al. (2008); Mielke y Vélez Pérez (2009).

De lo anterior, para el caso de que los axiones fríos desacoplados se comporten como un campo clásico, el campo del axión satisface la ecuación de Klein-Gordon.

\subsection{Comportamiento solitónico de halos esféricos}

Como los halos galácticos pueden considerarse esféricos en primera aproximación, se justifica la elección de una métrica tipo Schwarzschild en la cual el elemento de línea puede escribirse como

$$
d s^{2}=\mathrm{e}^{\nu(r)} d t^{2}-\mathrm{e}^{\lambda(r)}\left[d r^{2}+r^{2}\left(d \theta^{2}+\sin ^{2} \theta d \varphi^{2}\right)\right]
$$

donde $\nu=\nu(r)$ y $\lambda=\lambda(r)$ dependen del tipo de coordenada radial $r$ de Schwarzschild elegida. Considerando además un Ansatz para que el campo sea estacionario

$$
\phi(r, t)=P(r) \mathrm{e}^{-i \omega t}
$$

el cual describe un estado esféricamente simétrico para el campo escalar.

En estos términos, el tensor de energía-momento para el campo escalar simétricamente esférico es diagonal y toma una forma $T_{\mu}^{\nu}(\phi)=\operatorname{diag}\left(\rho,-p_{r},-p_{\perp},-p_{\perp}\right)$, donde

$$
\begin{aligned}
\rho & =\frac{1}{2}\left(m^{2} P^{2}+P^{2}+V\right), \\
p_{r} & =\rho-V, \\
p_{\perp} & =p_{r}-P^{\prime 2},
\end{aligned}
$$

además ${ }^{\prime}=d / d r$. La forma canónica de estas ecuaciones es análoga a la de un fluido perfecto, excepto que en general las presiones radial y tangencial son diferentes $p_{r} \neq p_{\perp}$ para un campo escalar. Esta anisotropía de la materia escalar es ignorada aunque en el espacio-tiempo plano la aproximación Newtoniana se mantiene. 
Los componentes principales no nulos de la ecuación de Einstein resultan ser

$$
\begin{aligned}
\nu^{\prime}+\lambda^{\prime} & =\kappa\left(\rho+p_{r}\right) r \mathrm{e}^{\lambda} \\
\lambda^{\prime} & =\kappa \rho r \mathrm{e}^{\lambda}-\frac{1}{r}\left(\mathrm{e}^{\lambda}-1\right)
\end{aligned}
$$

donde $\nu=\nu(r)$ y $\lambda=\lambda(r)$, es decir, las ecuaciones radiales. Las otras dos componentes no nulas satisfacen las identidades contraídas de Bianchi $\nabla^{\mu} T_{\mu}{ }^{\nu} \equiv 0$, de donde obtenemos

$$
\frac{d}{d r} p_{r}=-\nu^{\prime}\left[\rho+p_{r}-\frac{2}{r}\left(p_{r}-p_{\perp}\right)\right]
$$

una relación que es equivalente a la de equilibrio hidrostático de para fluido anisotrópicos, esta es una generalización de la ecuación de Tolman-Oppenheimer-Volkoff (TOV).

La ecuación diferencial para el campo escalar resulta

$$
P^{\prime \prime}(r)+\left(\frac{\nu^{\prime}-\lambda^{\prime}}{2}+\frac{2}{r}\right) P^{\prime}(r)+\mathrm{e}^{\lambda}\left(\mathrm{e}^{-\nu} \omega^{2}-\frac{d V(P)}{d P^{2}}\right) P(r)=0 .
$$

Aquí, se elige un modelo soluble analíticamente, pero de juguete, con un potencial de auto-interacción $\phi^{6}$, es decir, el potencial de Lane-Emden

$$
V(\phi)=\frac{m^{2}}{2}|\phi|^{2}\left(1-\chi \phi^{4}\right) \quad \chi|\phi|^{4} \leq 1
$$

donde $m$ es una pequeña masa, y $\chi$ una constante de acoplamiento, ambas caracterizando el hipotético campo escalar y que son pensadas como constantes de la naturaleza del mismo. Con este potencial se puede reescribir la ecuación de campo (3.5.6). La derivada del potencial puede escribirse ahora como

$$
\frac{d V(P)}{d P^{2}}=m^{2}-3 m^{2} \chi P^{4}
$$

donde $P(r)=\phi \mathrm{e}^{i m t}$, despejado del Ansatz estacionario. Para una configuración esférica de la ecuación no lineal de Klein-Gordon, eligiendo $\omega=m$, donde además se han aproximado $\mathrm{e}^{\lambda} \simeq \mathrm{e}^{\nu} \simeq 1$. Así, introduciendo (3.5.8) en (3.5.6) la ecuación se simplifica a una ecuación tipo Emden

$$
P^{\prime \prime}+\frac{2}{r} P^{\prime}+3 m^{2} \chi P^{5}=0
$$

que adimensionalizada es

$$
P^{\prime \prime}+\frac{2}{x} P^{\prime}+3 \chi P^{5}=0
$$

donde $x=m r$, es la coordenada adimensional radial. Para esta ecuación se conocen soluciones exactas de Emden completamente regulares (Figura 3.5.1)

$$
P(r)= \pm \chi^{-1 / 4} \sqrt{\frac{A}{1+A^{2} x^{2}}}
$$




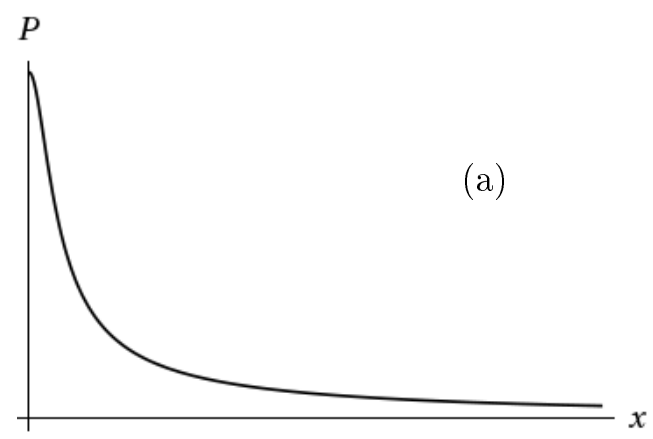

(b)

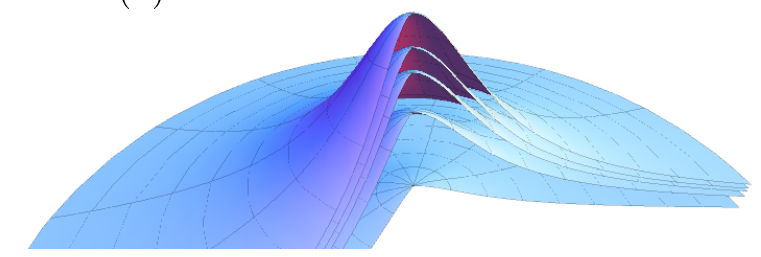

Figura 3.5.1: Solución de la ecuación de Lane-Emden que depende únicamente de la coordenada radial y de los parámetros $A<1$ y $\chi>0$. En la figura se muestra la variación de la función para valores de la constante de acoplamiento $\chi$.

donde $A=\sqrt{\chi} P^{2}(0)$ esta relacionada con el valor central. Solución que depende fuertemente de la constante de acoplamiento $\chi$, ya que el desarrollo en serie sería singular para $\lim _{\chi \rightarrow 0}$. Donde además restringimos que $A \leq 1$, lo que hace que el potencial permanezca positivo.

Este modelo en el espacio-tiempo plano, sirve para construir un solitón no topológico. En el caso de estrellas de bosones, con un enfoque de relatividad general para un potencial con auto-interacción $\lambda|\phi|^{4}$, se ha mostrado numéricamente que existe un solitón no topológico inclusive para valores negativos de $\lambda$; vid. Choi (2002); Bernal y Guzmán (2006); Palenzuela et al. (2007) estudiaron numéricamente la colisiones de estrellas de bosones descritas por el modelo y mostraron que existen dos diferentes regímenes: uno solitónico y otro de mezclado. Sin embargo, hay distintos tipos de estrellas de bosones con diferentes tipos de auto-interacciones no gravitacionales; vid. Schunck y Mielke (2003). 


\section{Capitulo 4}

\section{Klein-Gordon no lineal}

En la teoría de campo el modelo más simple de campo relativista es el campo escalar real libre, es un campo de una sola componente e invariante ante transformaciones del grupo de Poincaré. Para este campo la Lagrangiana puede escribirse como

$$
\mathcal{L}_{\mathrm{KG}}=\frac{1}{2}\left(\partial^{\mu} \phi\right)^{2}-\frac{1}{2} m^{2} \phi^{2}
$$

éste conduce a una ecuación de campo de la forma

$$
\left(\square+m^{2}\right) \phi(x)=0
$$

donde $\square \equiv \partial_{\mu} \partial^{\mu} \equiv c^{-2} \partial_{t}^{2}$, es el operador de D'Alembert y (4.0.2) es la famosa ecuación de Klein-Gordon. Esta ecuación es lineal y una de sus soluciones es una onda monocromática, la cual no es solitónica.

En el Lagrangiano de Klein-Gordon se ha utilizado un potencial $V(\phi)=m^{2} \phi^{2} / 2$, este potencial puede generalizarse sumando otro término además del cuadrático en $\phi$, con lo que se obtiene una ecuación de Klein-Gordon no lineal. Algunas de éstas, dan lugar a importantes modelos, como $\phi^{4}$ que incluye para masa imaginaria el modelo de Higgs. Otros términos conducen a modelos ya utilizados en el pasado, como en Lohe (1979) que utilizó modelos que incluyen términos de $\phi^{8}$ para mesones no masivos; o en Boulbitch (1997) que incluyendo términos de $\phi^{10}$ estudió la cristalización de proteinas quirales.

Incluir términos de orden superior a $\phi^{2}$ permite utilizar el modelo para explorar otras aplicaciones. En particular, hemos consideremos un modelo de juguete conocido como potencial de Lane-Emden. Estudiaremos en éste las soluciones solitónicas buscando una aplicación para halos de materia oscura y presentaremos parte de los resultados obtenidos y publicados en Castañeda Valle y Mielke (2013, 2014). 


\subsection{Ecuación de Klein-Gordon no lineal}

En la ecuación de Klein-Gordon no lineal, el comportamiento de onda lo brinda el operador D'Alembertiano; sin embargo, el verdadero comportamiento y restricciones lo dicta la elección de la función que constriñe la onda, o más particularmente la elección del potencial,

$$
\square \phi=-\frac{\partial V(\phi)}{\partial \phi} .
$$

Entre las muchas posible elecciones hemos estudiado un potencial de sine-Gordon, que condujo a la ecuación del mismo nombre

$$
\square \phi=-\frac{m^{3}}{\sqrt{\lambda}} \sin \left(\frac{\sqrt{\lambda}}{m} \phi\right),
$$

además es también el potencial efectivo que se obtuvo para el axión. El modelo que estudiaremos se puede considerar un caso intermedio entre Klein-Gordon y sine-Gordon. Para esto, expandiendo en potencias de la constante $\lambda$, el potencial de sine-Gordon (2.8.2)

$$
V_{\mathrm{SG}}(\phi)=\frac{m^{4}}{\lambda}\left[1-\cos \left(\frac{\sqrt{\lambda}}{m} \phi\right)\right]
$$

obtenemos

$$
V_{\mathrm{sG}}(\phi)=\frac{1}{2} m^{2} \phi^{2}-\frac{\lambda}{4 !} \phi^{4}+\frac{\lambda^{2}}{6 ! m^{2}} \phi^{6}-\frac{\lambda^{3}}{8 ! m^{4}} \phi^{8}+\ldots
$$

De este potencial, el Lagrangiano de Klein-Gordon (4.0.1) se consiguió eligiendo la aproximación de orden cero y despreciando los términos de orden superior $\mathcal{O}(\lambda)$, es decir

$$
V_{\mathrm{KG}}(\phi)=\frac{1}{2} m^{2} \phi^{2}
$$

si bien esta versión es muy importante en la teoría de partículas, es fácil obtener versiones más complejas y con mucha relevancia. El simple hecho de elegir los dos primeros términos y acomodando las constantes se puede obtener

$$
V_{\mathrm{H}}(\phi)=\frac{\lambda}{4}\left(\phi^{2}-\frac{\mu^{2}}{\lambda}\right)^{2},
$$

que genera un término de $\phi^{4}$ en el Lagrangiano. Dicho término representa la autointeracción del campo y conduce a una ecuación que no es lineal. Éste es el famoso modelo de Higgs, que conduce al bosón de Higgs recientemente descubierto y que está estrechamente relacionado con la masa de las partículas.

De manera similar eligiendo el primer y tercer términos de (4.1.4) y acomodando las constantes obtenemos el potencial de Lane-Emden, que escrito para un campo real es

$$
V_{\mathrm{LE}}(\phi)=\frac{m^{2}}{2} \phi^{2}\left(1-\chi \phi^{4}\right) \text {. }
$$



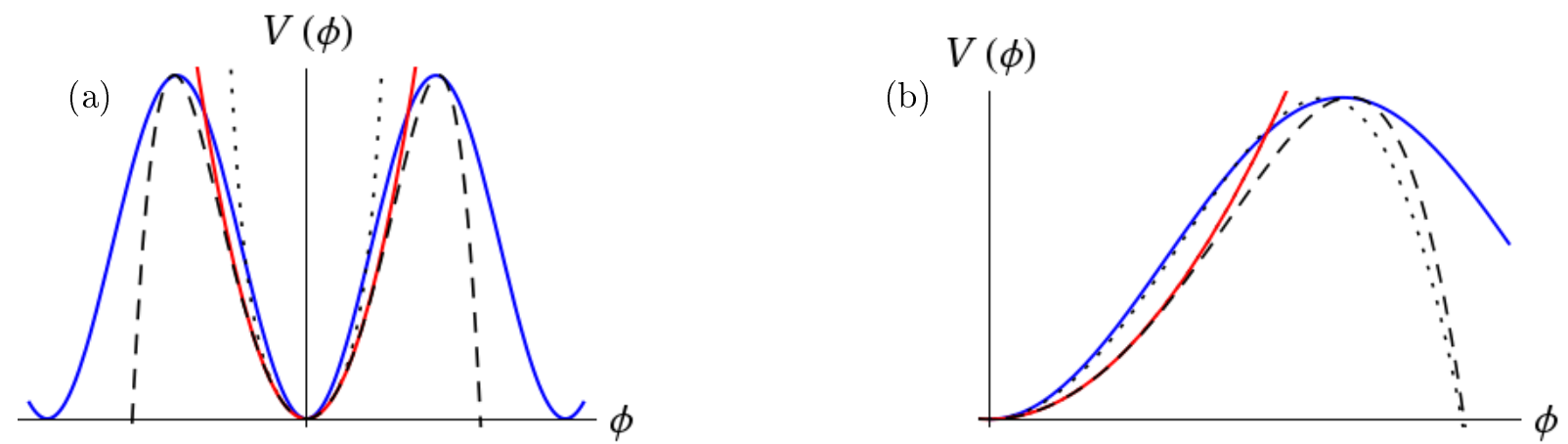

Figura 4.1.1: En la Figura se muestran potenciales para distintos casos del potencial efectivo del axión: masivo $\phi^{2}$ (rojo), $\phi^{4}$ (punteada), $\phi^{6}$ (segmentada) y axión (azul).

En la Figura 4.1.1, se grafican distintos casos del potencial: $\phi^{4}$ (línea negra, punteada) el potencial del axión (azul, sólida), la aproximación $\phi^{6}$ y el potencial de Klein-Gordon (roja); Figura 4.1.1b, tienen un comportamiento similar cerca del origen $\phi=0$, más importante aún es que la aproximación de Lane-Emden nos permitirá soluciones solitónicas o tipo lump.

\subsection{Modelo $\phi^{6}$ : Materia oscura escalar}

La primera propuesta de que la materia oscura podría ser un campo escalar fue realizada por Schunck (1998), usando un campo escalar complejo acoplado a las ecuaciones de Einstein. Idea que ha sido explorada por varios autores con aplicaciones generales de materia oscura y se ha establecido como materia oscura de campo escalar o SFDM (Scalar Field Dark Matter). Modelo que para estrellas de bosones ha obtenido resultados prometedores; vid. Lee (2009). Seguido a la propuesta de Schunck, Spergel y Steinhardt (2000) propusieron que la materia oscura era interactuante.

Un modelo de juguete propuesto por Mielke (1978) considera una autointeracción repulsiva $\phi^{6}$. El cual, para el caso límite de espacio plano en simetría esférica proporciona soluciones tipo solitón exactas, correspondientes a una ecuación no lineal de Klein-Gordon. Este modelo proporciona curvas de rotación cualitativamente correctas para un halo de materia oscura; vid. Mielke y Schunck (2002); Mielke y Vélez Pérez (2009).

\subsubsection{Ecuación de Lane-Emden en astrofísica}

Un medio politrópico es aquel en el cual la densidad y la presión responden a la relación

$$
p=K \rho^{(n+1) / n}
$$


donde $K$ y $n$ son constantes, ésta última es referida como índice politrópico. La ecuación fue utilizada por Lane, Ritter, Kelvin, Emden y Fowler para estudiar esferas de gas politrópico; vid. Chandrasekhar (1958). Se utiliza para modelar las capas convectiva y radiativa interna en el Sol o en estrellas similares. Relacionando la conservación de masa radial

$$
\frac{d M(r)}{d r}=4 \pi r^{2} \rho(r)
$$

y la conservación de momento en el límite de velocidad cero de la ecuación de TOV, es

$$
\frac{d p(r)}{d r}=-\frac{G M(r) \rho(r)}{r^{2}} .
$$

Se puede obtener una ecuación de Poisson para una esfera autogravitante

$$
\frac{1}{r^{2}} \frac{d}{d r}\left(\frac{r^{2}}{\rho(r)} \frac{d p(r)}{d r}\right)=-4 G \rho(r) .
$$

La cual al considerar la relación para el politrópico (4.2.1) se puede reducir a una sola función de $r$. Además de los valores centrales de la presión y densidad, respectivamente, $p_{c}$ y $\rho_{c}$; se puede obtener

$$
\frac{p(r)}{\rho(r)^{(n+1) / n}}=\frac{p_{c}}{\rho_{c}^{(n+1) / n}} .
$$

Para simplificar (4.2.4), se introduce una función adimensional de Lane-Emden $\theta$, con la que la densidad y presión pueden escribirse como

$$
\begin{aligned}
\theta^{n} & =\frac{\rho(r)}{\rho_{c}} \\
\theta^{n+1} & =\frac{p(r)}{p_{c}} .
\end{aligned}
$$

Sustituyendo estas expresiones en (4.2.4), se puede reducir a

$$
\frac{(n+1) p_{c}}{4 \pi G \rho_{c}^{2}}\left(\frac{1}{r^{2}}\right) \frac{d}{d r}\left[r^{2} \frac{d \theta}{d r}\right]=-\theta^{n},
$$

en ésta, las constantes, incluido el índice politrópico, representan una longitud al cuadrado, conocida como longitud de Emden

$$
r_{0}^{2}=\frac{(n+1) p_{c}}{4 \pi G \rho_{c}^{2}} .
$$

Con esto podemos adimensionalizar (4.2.7), escribiendo $\xi=r / r_{0}$, con lo que resulta

$$
\frac{1}{\xi^{2}} \frac{d}{d \xi}\left[\xi^{2} \frac{d \theta}{d \xi}\right]=-\theta^{n}
$$

que es la ecuación diferencial de Lane-Emden. Esta ecuación diferencial ordinaria de segundo orden se puede resolver analíticamente para los casos $n=0,1,5$; otros casos se resuelven numéricamente; vid. Mullan (2010). 


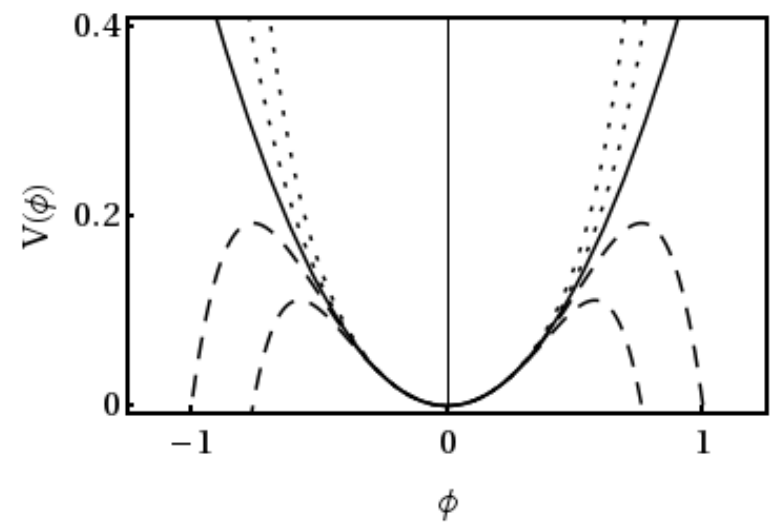

Figura 4.3.1: Potencial $\tilde{V}(\phi)=\phi^{2}\left(1-\chi \phi^{4}\right)=\tilde{V}(-\phi)$. Para cualquier valor de $\chi$ obtenemos un mínimo local, pero para $\chi=0$ obtenemos un potencial cuadrático (línea sólida); para valores de $\chi<0$ obtenemos parábolas (líneas punteadas), mientras par valores $\chi>0$ obtenemos un potencial que tiene además dos máximos (líneas segmentadas).

\subsection{Potencial tipo Lane-Emden}

El modelo de Lane-Emden (LE) se logra al considerar en el potencial los términos $\phi^{2}$ y $\phi^{6}$ que con un término de acoplamiento puede escribirse como

$$
V_{\mathrm{LE}}(\phi)=\frac{m^{2}}{2} \phi^{2}\left(1-\chi \phi^{4}\right)
$$

donde $m$ es la masa, $\chi$ es una constante de acoplamiento. La forma del potencial depende del parámetro $\chi$, para $\chi \leq 0$ tiene solo un mínimo, mientras que con $\chi>0$ el potencial tiene además dos máximos locales; nosotros consideraremos valores $\chi>0$, éste se asemejará más al potencial de sine-Gordon; Figura 4.3.1.

Introduciendo este potencial en la ecuación no lineal de Klein-Gordon (4.1.1), obtenemos

$$
\left(\frac{1}{c^{2}} \frac{\partial^{2}}{\partial t^{2}}-\nabla \cdot \nabla\right) \phi=-m^{2} \phi\left(1-3 \chi \phi^{4}\right) .
$$

Utilizando el Ansatz estacionario y de simetría esférica

$$
\phi(r, t)=P(r) \mathrm{e}^{-i \omega t}
$$

la ecuación toma la forma

$$
\frac{d V_{\mathrm{LE}}(P)}{d P^{2}}=m^{2}-3 m^{2} \chi P^{4}
$$

que puede escribirse como

$$
P^{\prime \prime}+\frac{2}{\xi} P^{\prime}+3 \chi P^{5}=0
$$

donde $\xi=m r \mathrm{y}^{\prime} \equiv d / d \xi$; lo que es precisamente la ecuación de Lane-Emden (4.2.9) para un índice politrópico $n=5$. 

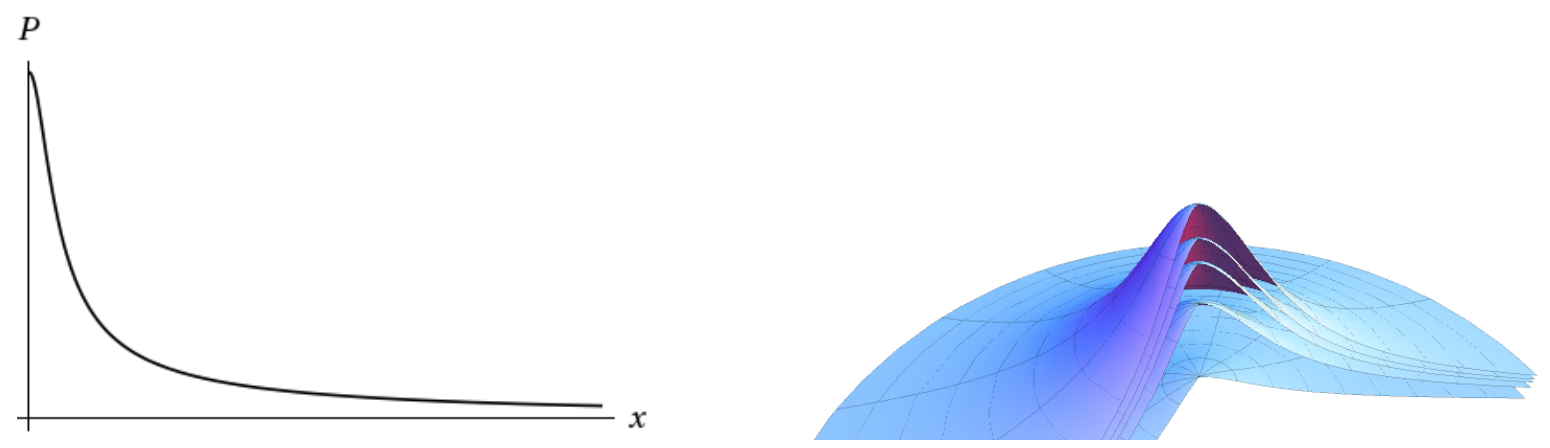

Figura 4.3.2: Solución de la ecuación Lane-Emden que depende únicamente de la coordenada radial y de los parámetros $A<1$ y $\chi>0$. En la figura se muestra la variación de la función para valores de la constante de acoplamiento $\chi$.

Esta ecuación tiene una solución exacta de Emden completamente regular (Figura 4.3 .2$)$

$$
P(\xi)= \pm \chi-1 / 4 \sqrt{\frac{A}{1+A^{2} \xi^{2}}}
$$

donde $A=\sqrt{\chi} P^{2}(0)$ está asociada con el valor central. Notamos que la función es decreciente y tiende a cero mientras $\xi \rightarrow \infty$ lo que significa que la correspondiente configuración de equilibrio se extiende hasta infinito.

\subsection{Soluciones solitónicas en $(1+1) \mathrm{D}$}

La ecuación de Klein-Gordon no lineal con el potencial de Lane-Emden pueden generar soluciones en $3+1$ dimensiones con simetría esférica. Buscaremos ahora soluciones solitónicas en sistemas $1+1$ dimensionales, aplicando lo expuesto en el Capítulo 2.

\subsubsection{Solitón simple}

Partiendo de la ecuación general (4.1.1) e introduciendo el potencial de Lane-Emden (4.3.1), obtenemos la ecuación de onda (4.3.2), que en $1+1$ dimensiones se reduce a

$$
\left(\frac{1}{c^{2}} \frac{\partial^{2}}{\partial t^{2}}-\frac{\partial^{2}}{\partial x^{2}}\right) \phi=-\frac{m^{2}}{2} \phi^{2}\left(1-\chi \phi^{4}\right)
$$

en ésta, podemos reescalar las coordenadas utilizando la masa, $(\tilde{t}, \tilde{x})=m(t, x)$; con lo que escribimos

$$
\left(\frac{\partial^{2}}{\partial \tilde{x}^{2}}-\frac{1}{c^{2}} \frac{\partial^{2}}{\partial \tilde{t}^{2}}\right) \phi=\phi\left(1-3 \chi \phi^{4}\right) .
$$

De aquí, para obtener la solución reescribamos (4.4.2) como

$$
\left(\frac{\partial^{2}}{\partial \tilde{x}^{2}}-\frac{1}{c^{2}} \frac{\partial^{2}}{\partial \tilde{t}^{2}}\right) \phi=\frac{\partial \tilde{V}_{\mathrm{LE}}(\phi)}{\partial \phi}
$$


con $\tilde{V}_{\mathrm{LE}}(\phi)=\frac{1}{2} \phi^{2}\left(1-\chi \phi^{4}\right)$, consideremos además la ecuación estática, y multiplicando por el factor integrante $\partial \phi / \partial \tilde{x}$, tenemos

$$
\begin{aligned}
\frac{\partial^{2} \phi}{\partial \tilde{x}^{2}} & =\frac{\partial \tilde{V}(\phi)}{\partial \phi} \\
\frac{\partial^{2} \phi}{\partial \tilde{x}^{2}} \frac{d \phi}{d \tilde{x}} & =\frac{\partial \tilde{V}(\phi)}{\partial \phi} \frac{d \phi}{d \tilde{x}} \\
\frac{1}{2} \frac{d}{d \tilde{x}}\left(\frac{d \phi}{d \tilde{x}}\right)^{2} & =\frac{\partial \tilde{V}(\phi)}{\partial \tilde{x}}
\end{aligned}
$$

integrando encontramos

$$
\begin{aligned}
\frac{d \phi}{d \tilde{x}} & = \pm \sqrt{2 \tilde{V}(\phi)+k_{1}} \\
\int d \tilde{x} & = \pm \int \frac{d \phi}{\sqrt{2 \tilde{V}(\phi)+k_{1}}}
\end{aligned}
$$

que corresponde a las ecuaciones de Bogomol'nyi (2.5.3) con $k_{1}=0$. El hecho de que el potencial tenga un solo mínimo en $\phi=0$, implica una solución tipo lump, es decir, no topológica. Integrando una segunda vez e introduciendo la forma del potencial $\tilde{V}(\phi)$, tenemos que

$$
\tilde{x}= \pm \int \frac{d \phi}{\sqrt{2 \tilde{V}(\phi)}}= \pm \int \frac{d \phi}{\sqrt{\phi^{2}\left(1-\chi \phi^{4}\right)}}=\mp \frac{1}{2} \operatorname{arcsech}\left(\sqrt{\chi} \phi^{2}\right)+k_{2}
$$

aquí surge una condición más, ya que para tener valores reales se debe cumplir que $\chi \phi^{4}<$ 1, como muestra la Figura 4.3.1.

Invirtiendo la ecuación para $\phi$, obtenemos las ecuaciones

$$
\begin{aligned}
\chi^{1 / 2} \phi^{2} & =\operatorname{sech}\left[\mp 2\left(\tilde{x}-k_{2}\right)\right] \\
\phi & = \pm \frac{1}{\sqrt[4]{\chi}} \sqrt{\operatorname{sech}[2 \tilde{x}]}
\end{aligned}
$$

en ésta, la constante $k_{2}$ puede asociarse a la posición inicial del solitón por lo que podemos hacer $k_{2}=0$. Aplicando un boost de Lorentz la solución toma la forma

$$
\phi(\tilde{t}, \tilde{x})= \pm \frac{1}{\sqrt[4]{\chi}} \sqrt{\operatorname{sech}\left[2 \gamma\left(\tilde{x}-\tilde{x}_{0}-v \tilde{t}\right)\right]}
$$

tanto la simetría $\mathbb{Z}^{2}$ de $\phi$, del potencial como la (4.4.8) permiten la existencia de dos soluciones, ya sea para $\phi>0$ o $\phi<0$; Figura 4.4.1.

Utilizando esto podemos obtener la densidad de energía (Figura 4.4.2) que resulta en

$$
\rho(\tilde{t}, \tilde{x})=\frac{m^{2} \gamma^{2}}{\chi^{1 / 2}} \operatorname{sech}\left[2 \gamma\left(\tilde{x}-\tilde{x}_{0}-v \tilde{t}\right)\right] \tanh ^{2}\left[2 \gamma\left(\tilde{x}-\tilde{x}_{0}-v \tilde{t}\right)\right] .
$$



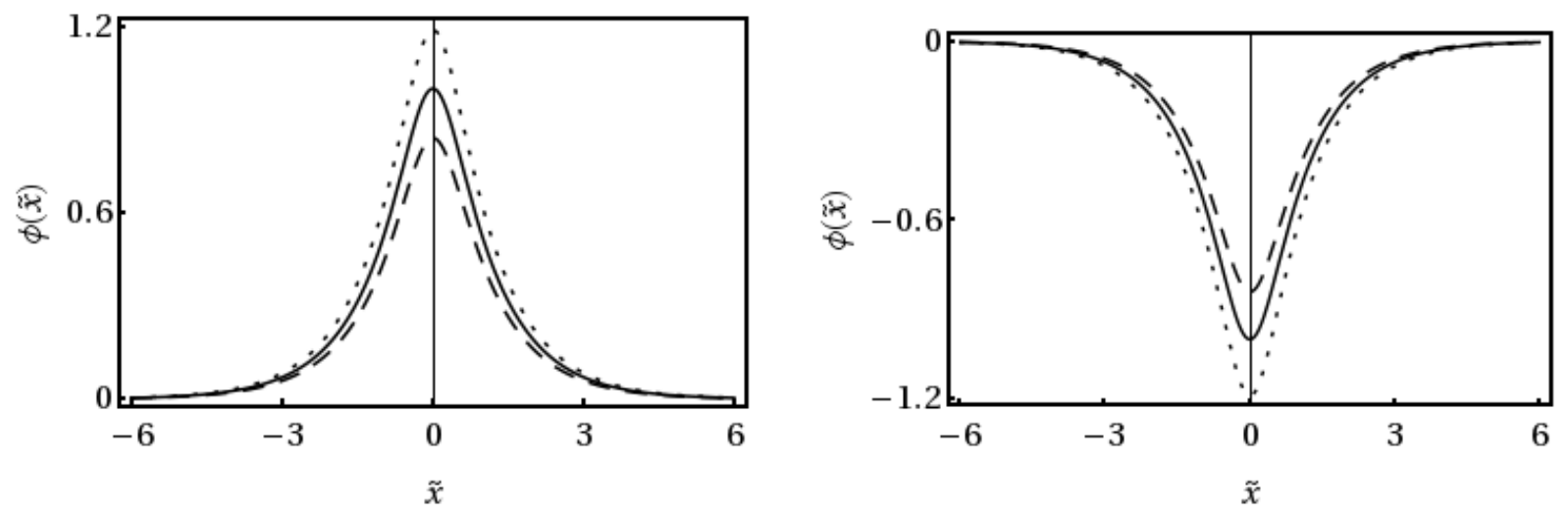

Figura 4.4.1: Solución tipo lump centrada en $\tilde{x}_{o}=0$, aquí las soluciones para tres valores de $\chi$, $\chi=0.5$ la línea punteada, $\chi=1$ la línea sólida y $\chi=2$ la línea segmentada.

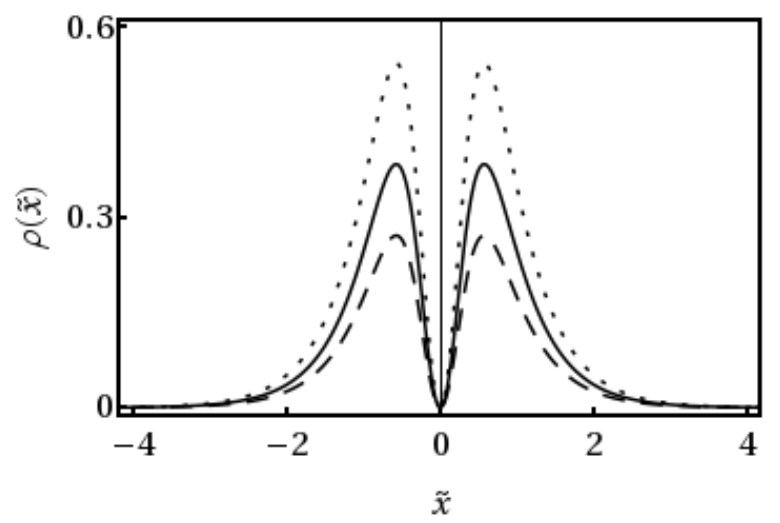

Figura 4.4.2: Densidad de energía $\tilde{\rho}(x)=\chi^{-1 / 2}\left\{\operatorname{sech}\left(2\left(\tilde{x}-\tilde{x}_{o}\right)\right)-\operatorname{sech}^{3}\left(2\left(\tilde{x}-\tilde{x}_{o}\right)\right)\right\}$, centrada en $x_{0}=0$, para tres valores de $\chi, \chi=0.5$ la línea punteada, $\chi=1$ la línea sólida y $\chi=2$ la línea segmentada.

Similarmente podemos integrar y obtener el superpotencial

$$
W= \pm m \int \phi \sqrt{1-\chi \phi^{4}} d \phi
$$

lo que resulta en (Figura 4.4.3)

$$
W(\phi)=\mp \frac{m}{4}\left[\frac{1}{\chi^{1 / 2}} \arcsin \left(\chi^{1 / 2} \phi^{2}\right)+\phi^{2} \sqrt{1-\chi \phi^{4}}\right]
$$

Integrando las correspondientes ecuaciones encontramos la energía. De la densidad obtenemos (Figura 4.4.4)

$$
\begin{aligned}
E_{\text {lump }} & =\int_{-\infty}^{\infty} \rho(t, x) d x=m \int_{-\infty}^{\infty} \rho(\tilde{t}, \tilde{x}) d \tilde{x} \\
& =\frac{1}{4} \frac{m \gamma}{\sqrt{\chi}}\left[\frac{\sinh [2 \gamma(v \tilde{t}-\tilde{x})]}{\cosh ^{2}[2 \gamma(v \tilde{t}-\tilde{x})]}-2 \arctan [2 \gamma(v \tilde{t}-\tilde{x})]\right]_{-\infty}^{\infty}
\end{aligned}
$$



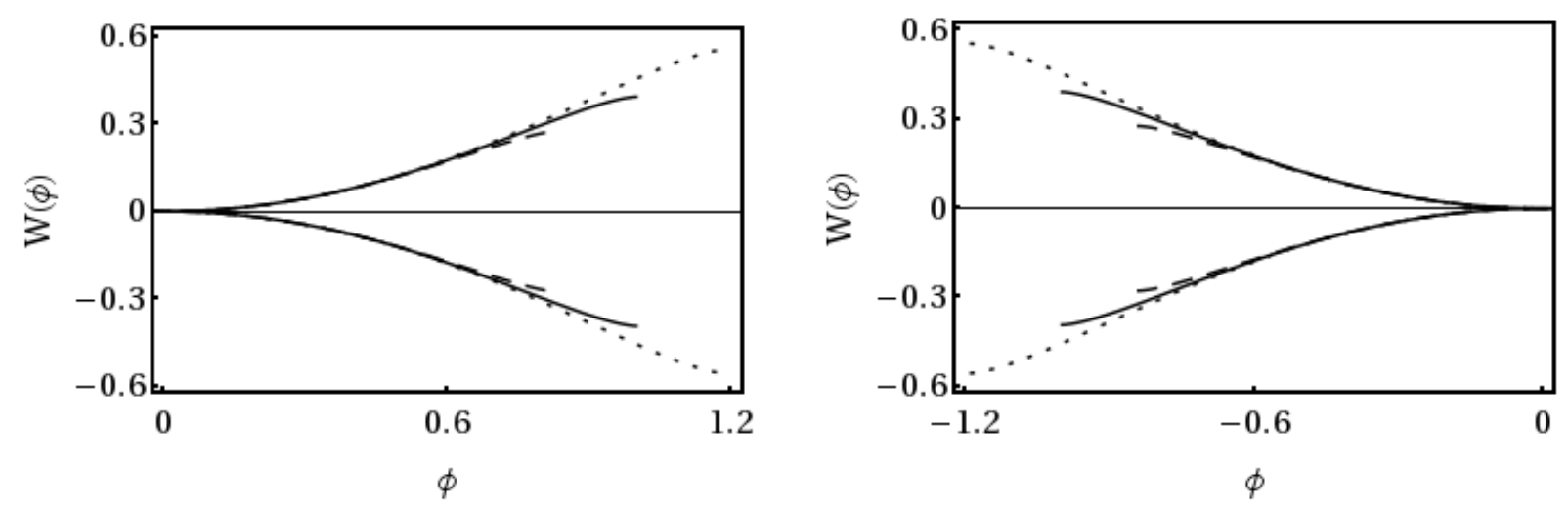

Figura 4.4.3: Superpotenciales $\tilde{W}(\phi)=\mp \frac{1}{4}\left(\chi^{-1 / 2} \arcsin \left(\chi^{1 / 2} \phi^{2}\right)+\phi^{2} \sqrt{1-\chi \phi^{4}}\right)$ para $\phi>0$ y $\phi<0$, aquí las soluciones para tres valores de $\chi, \chi=0.5$ la línea punteada, $\chi=1$ la línea sólida y $\chi=2$ la línea segmentada.

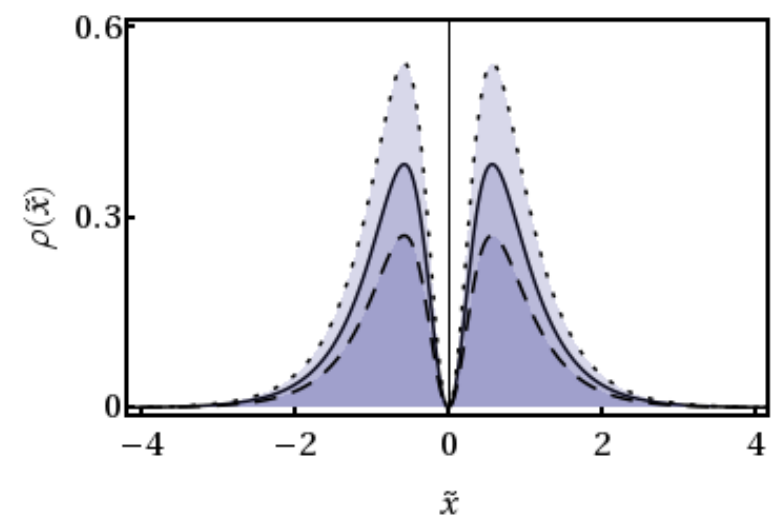

Figura 4.4.4: Energía calculada utilizando la densidad de energía $\tilde{\rho}(x)$ centrada en $x_{o}$, aquí las soluciones para tres valores de $\chi, \chi=0.5$ la línea punteada, $\chi=1$ la línea sólida y $\chi=2$ la línea segmentada.

Mientras que del superpotencial vemos que la solución $\phi(\tilde{x})$ es, ya sea positiva o negativa, según el intervalo de $\phi$ de $\left[0, \pm \chi^{-1 / 4}\right)$, por lo que podemos tomar dos trozos para la energía (Figura 4.4.5),

$$
\begin{aligned}
E_{\text {lump }} & =\left|W(\phi(-\infty))-W\left(\phi\left(\tilde{x}_{o}\right)\right)\right|+\left|W\left(\phi\left(\tilde{x}_{o}\right)\right)-W(\phi(\infty))\right| \\
& =2\left|W\left(\phi\left(\tilde{x}_{o}\right)\right)-W(\phi(\infty))\right| \\
& =2\left|W\left(\chi^{-1 / 4}\right)-W(0)\right|
\end{aligned}
$$

Para cualquiera de los casos obtenemos que la energía para el lump de Lane-Emden es

$$
E_{\text {lump }}=\frac{m}{4} \frac{\pi}{\chi^{1 / 2}}
$$



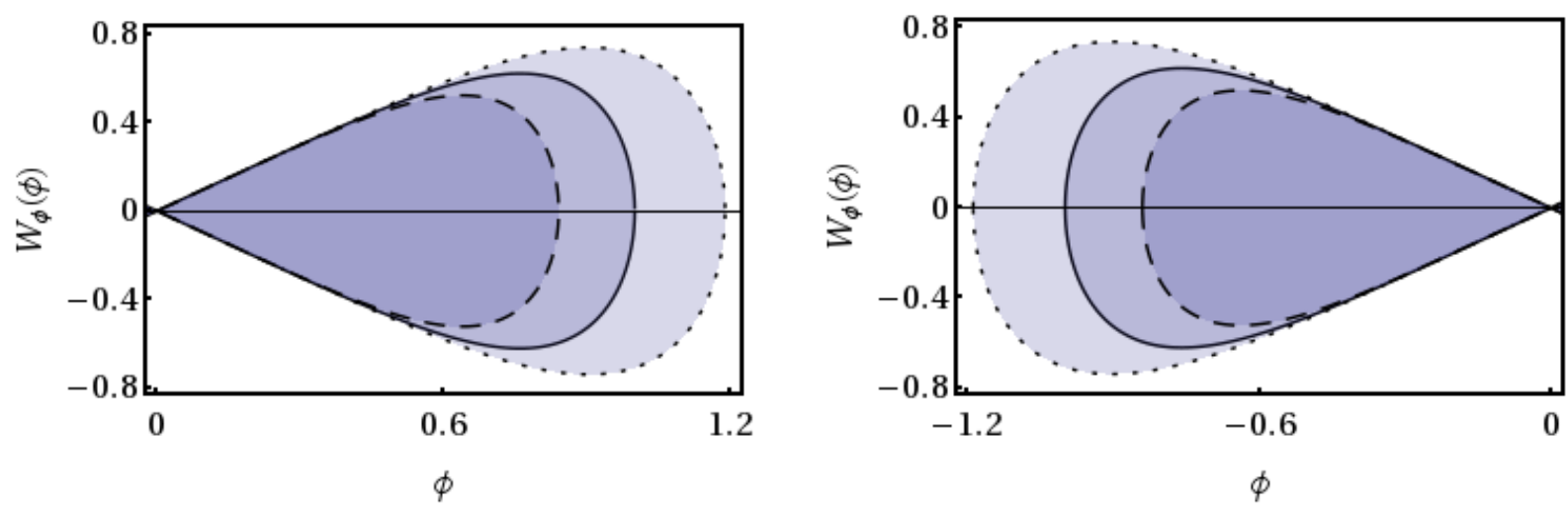

Figura 4.4.5: Energía calculada utilizando $\tilde{W}_{\phi}(\phi)= \pm \phi \sqrt{1-\chi \phi^{4}}$ para $\phi>0$ y $\phi<0$, aquí las soluciones para tres valores de $\chi, \chi=0.5$ la línea punteada, $\chi=1$ la línea sólida y $\chi=2$ la línea segmentada.

\subsubsection{Solitón múltiple}

Hemos obtenido la solución 1-solitónica ahora busquemos un solitón múltiple utilizando la transformación de Bäcklund; que en esencia es la combinación no lineal de dos soluciones.

\subsubsection{Transformaciones de Bäcklund}

Considerando el potencial tipo Lane-Emden (3.5.7) y el reescalamiento de las coordenadas partamos de la ecuación (4.4.2) para calcular la transformación. En el lado izquierdo, el operador D'Alembertiano puede escribirse en coordenadas de cono de luz, (vid. Sección 2.7) definidas por

$$
\xi=\frac{1}{2}(\tilde{x}+c \tilde{t}) \quad \eta=\frac{1}{2}(\tilde{x}-c \tilde{t})
$$

entonces la ecuación de campo resultante es

$$
\phi_{\xi \eta}=\phi\left(1-3 \chi \phi^{4}\right)
$$

y las transformaciones de Bäcklund (2.7.15) quedan

$$
\begin{aligned}
\phi_{\xi} & = \pm \frac{\sqrt{\phi^{2}\left(1-\chi \phi^{4}\right)+c_{2}}}{a} \\
\phi_{\eta} & = \pm a \sqrt{\phi^{2}\left(1-\chi \phi^{4}\right)+c_{2}}
\end{aligned}
$$

donde $a$ es un parámetro, además tomando $c_{2}=0$, e integrando implícitamente obtenemos

$$
\begin{aligned}
\pm \int \frac{d \phi}{\phi \sqrt{1-\chi \phi^{4}}} & =a \eta+\frac{1}{a} \xi+c_{3} \\
\mp \frac{1}{2} \operatorname{arcsech}\left(\sqrt{\chi} \phi^{2}\right) & =\zeta+c_{3}
\end{aligned}
$$


donde hemos escrito $\zeta=a \eta+\xi / a$; que invirtiendo tenemos

$$
\phi= \pm \frac{1}{\sqrt[4]{\chi}} \sqrt{\operatorname{sech}\left[ \pm 2\left(\zeta+c_{3}\right)\right]},
$$

esta ecuación es idéntica a (4.4.8) con $x_{0}=c_{3}=0$. Para notarlo se regresa a las coordenadas originales $(\tilde{t}, \tilde{x})$, tal como en la Sección 2.8.3,

$$
\zeta=\frac{a+a^{-1}}{2}\left(\tilde{x}+c \frac{1-a^{2}}{1+a^{2}} \tilde{t}\right)=\gamma(\tilde{x}+v \tilde{t}) .
$$

Ahora la transformación de Bäcklund para dos soluciones $\phi^{(0)}$ y $\phi^{(1)}$ se puede escribir usando (2.7.32) como

$$
\begin{aligned}
& \left(\phi^{(1)}+\alpha \phi^{(0)}\right)_{\xi}= \pm \frac{1}{a}\left(\phi^{(1)}-\alpha \phi^{(0)}\right) \sqrt{1-\chi\left(\phi^{(1)}-\alpha \phi^{(0)}\right)^{4}} \\
& \left(\phi^{(1)}-\alpha \phi^{(0)}\right)_{\eta}= \pm a\left(\phi^{(1)}+\alpha \phi^{(0)}\right) \sqrt{1-\chi\left(\phi^{(1)}+\alpha \phi^{(0)}\right)^{4}}
\end{aligned}
$$

Planteando el teorema de Bianchi correspondiente para obtener una forma totalmente algebraica para las soluciones $\phi^{(i)}, i=0,1,2,3$

$$
\phi^{(3)}=B_{2} \phi^{(1)}=B_{1} \phi^{(2)}=B_{2} B_{1} \phi^{(0)}=B_{1} B_{2} \phi^{(0)}
$$

obtenemos

$$
\begin{aligned}
& a_{1}\left\{\left(\phi^{(3)}-\alpha \phi^{(1)}\right) \sqrt{1-\chi\left(\phi^{(3)}-\alpha \phi^{(1)}\right)^{4}}-\alpha\left(\phi^{(2)}-\alpha \phi^{(0)}\right) \sqrt{1-\chi\left(\phi^{(2)}-\alpha \phi^{(0)}\right)^{4}}\right\} \\
& \quad=a_{2}\left\{\left(\phi^{(3)}-\alpha \phi^{(2)}\right) \sqrt{1-\chi\left(\phi^{(3)}-\alpha \phi^{(2)}\right)^{4}}-\alpha\left(\phi^{(1)}-\alpha \phi^{(0)}\right) \sqrt{1-\chi\left(\phi^{(1)}-\alpha \phi^{(0)}\right)^{4}}\right\}
\end{aligned}
$$

y debemos resolver esta ecuación para $\phi^{(3)}$, partiendo de una solución para $\phi^{(0)}=0$ además de $\phi^{(1)}$ y $\phi^{(2)}$ dos solitones de la forma (4.4.19), tenemos entonces

$$
\begin{gathered}
a_{1}\left(\phi^{(3)}+\alpha \phi^{(2)}\right) \sqrt{1-\chi\left(\phi^{(3)}+\alpha \phi^{(2)}\right)^{4}}-a_{2}\left(\phi^{(3)}+\alpha \phi^{(1)}\right) \sqrt{1-\chi\left(\phi^{(3)}+\alpha \phi^{(1)}\right)^{4}} \\
=\alpha\left\{a_{1} \phi^{(1)} \sqrt{1-\chi\left(\phi^{(1)}\right)^{4}}-a_{2} \phi^{(2)} \sqrt{1-\chi\left(\phi^{(2)}\right)^{4}}\right\}
\end{gathered}
$$

una forma aún difícil de resolver analíticamente, de hecho se plantea que para que exista una forma soluble, el potencial debe satisfacer (2.7.2), que en este caso resulta en

$$
1-15 \chi \phi^{4}+\frac{1}{2} k \phi^{2}\left(1-\chi \phi^{4}\right)=0
$$

donde $k$ debe ser una constante. 


\subsubsection{Aproximación a la transformación de Bäcklund $(1+1) \mathrm{D}$}

Si bien para la ecuación que nos interesa (4.4.1), no se puede definir una auto-transformación para el solitón múltiple, sí se puede definir una transformación generalizada que por el momento se ha utilizado como guía para probar la validez de las aproximaciones consideradas.

Las transformaciones de Bäcklund de la ecuación de sine-Gordon se pueden obtener de una manera exacta y están definidas en términos de la misma función seno. Utilizado la filosofía planteada por Makhankov (1978) para aproximar la solución en el modelo $\phi^{4}$, planteamos una aproximación de la solución (4.4.24), utilizando las transformaciones de Bäcklund de la ecuación de sine-Gordon, para resolver colisiones de solitones (solitones múltiples) para potencial $\phi^{6}$.

La aproximación se puede obtener al notar que para la ecuación de sine-Gordon en coordenadas cono de luz

$$
\tilde{\theta}_{\xi \eta}=\sin \tilde{\theta}
$$

la solución encontrada es

$$
\tilde{\theta}_{\mathrm{SG}}=4 C \arctan \left(\mathrm{e}^{\gamma(\tilde{x}-u \tilde{t})}\right)=4 C \arctan \left(\mathrm{e}^{\zeta}\right)
$$

en ésta se han redefinido las variables, además de escribir $\zeta=\gamma(\tilde{x}-v \tilde{t})$, donde $v$ es la velocidad del solitón y $\gamma$ es el factor de Lorentz. Notando que la solución $\tilde{\theta}_{\mathrm{sG}}$ se puede transformar en la solución (4.4.19) con $b=0$, vía la transformación

$$
\psi(\tilde{\theta})=\frac{1}{\chi^{1 / 4}}\left(\sin \frac{\tilde{\theta}_{\mathrm{sG}}}{2}\right)^{1 / 2}=\frac{1}{\chi^{1 / 4}}(\operatorname{sech} \zeta)^{1 / 2}=\phi
$$

transformación que en este caso provee una solución exacta para $\psi(\tilde{\theta})$.

Ahora consideramos el solitón múltiple para sine-Gordon, en particular la colisión de un kink y un antikink tiene la forma

$$
\tilde{\theta}=4 \arctan \left(\mathbb{B} \frac{\sinh \left(\frac{\zeta_{1}-\zeta_{2}}{2}\right)}{\cosh \left(\frac{\zeta_{1}+\zeta_{2}}{2}\right)}\right)
$$

donde

$$
\mathbb{B}=\frac{a_{1}+a_{2}}{a_{1}-a_{2}}
$$

donde $a_{1}$ y $a_{2}$ son los parámetros de las transformaciones de Bäcklund correspondientes a las velocidades $v_{1}$ y $v_{2}$, de cada solitón que conducen a la vez a $\gamma_{1}$ y $\gamma_{2}$. 

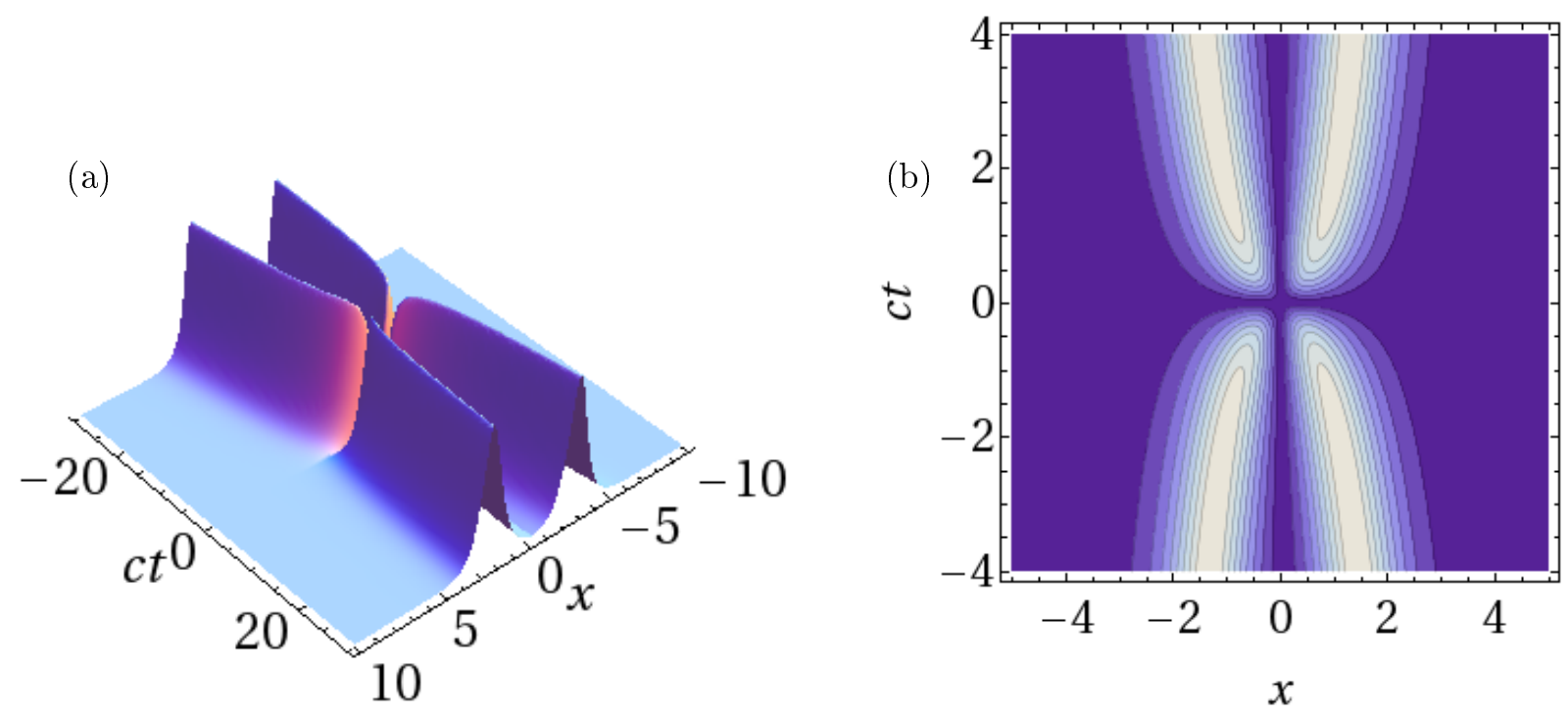

Figura 4.4.6: Derivada de la solución $\theta_{\mathrm{k} \overline{\mathrm{k}}}$ en espacio-tiempo. La gráfica permite observar cómo en el punto de colisión las funciones se anulan y posteriormente se recuperan, mostrando el desfase correspondiente a la colisión.

\subsubsection{Comparación}

En la Figura 4.4.6, se muestra el valor absoluto de la derivada de la función que nos provee una forma más comparable, es decir, más localizable respecto a un solitón que transita entre sectores topológicos.

Aplicando la transformación obtenemos

$$
\psi(\theta)=\frac{1}{\sqrt[4]{\chi}}\left(\sin \left\{2 \arctan \left[\mathbb{B} \frac{\sinh \left(\frac{\zeta_{1}-\zeta_{2}}{2}\right)}{\cosh \left(\frac{\zeta_{1}+\zeta_{2}}{2}\right)}\right]\right\}\right)^{1 / 2}=\frac{1}{\sqrt[4]{\chi}}(\operatorname{sech} \tilde{k})^{1 / 2}
$$

con $\mathbb{B}$ dada por en $(4.4 .30)$ y

$$
\tilde{k}=\ln \left[\mathbb{B} \frac{\sinh \left(\frac{\zeta_{1}-\zeta_{2}}{2}\right)}{\cosh \left(\frac{\zeta_{1}+\zeta_{2}}{2}\right)}\right]=\ln \left[\mathbb{B} \frac{\exp \zeta_{1}+C \exp \zeta_{2}}{\exp \left(\zeta_{1}+\zeta_{2}\right)-C}\right]
$$

considerando la expresión completa

$$
\begin{aligned}
\psi(\theta) & =\frac{1}{\sqrt[4]{\chi}}\left(\operatorname{sech} \ln \left[\mathbb{B} \frac{\exp \zeta_{1}+C \exp \zeta_{2}}{\exp \left(\zeta_{1}+\zeta_{2}\right)-C}\right]\right)^{1 / 2} \\
& =\frac{1}{\sqrt[4]{\chi}}\left(\frac{2 \mathbb{B}\left(\sinh \zeta_{1}-\sinh \zeta_{2}\right)}{\mathbb{B}^{2}\left\{\cosh \left(\zeta_{1}-\zeta_{2}\right)+C\right\}+\left\{\cosh \left(\zeta_{1}+\zeta_{2}\right)-C\right\}}\right)^{1 / 2}
\end{aligned}
$$

en la Figura 4.4.7 se muestra que los solitones se comportan como era de esperarse tras la colisión. Se puede señalar a favor qué: la velocidad se comporta de una manera adecuada, 

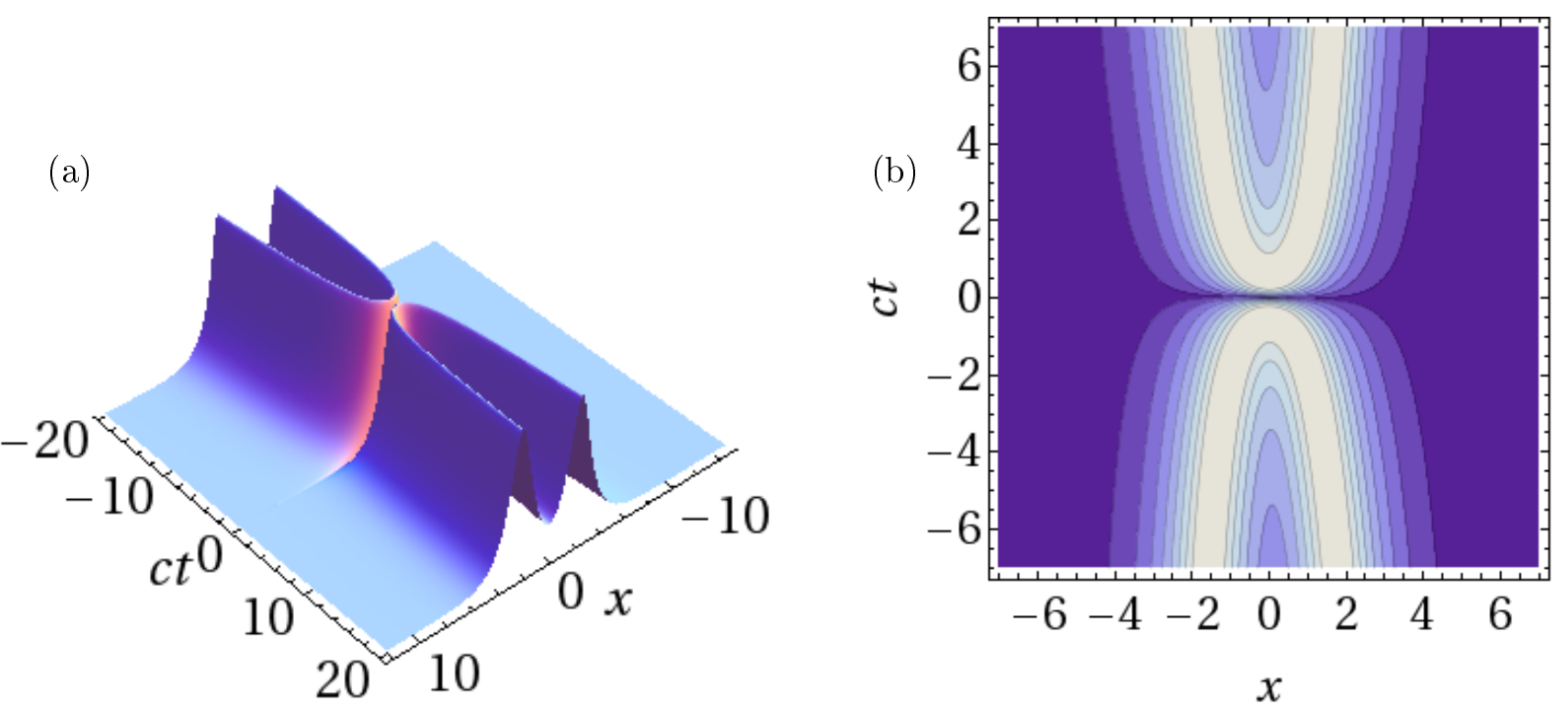

Figura 4.4.7: Aproximación al solitón múltiple de $\phi^{6}$.

ya que después de la colisión se recupera la original; además que el desfase espaciotemporal resultante de la colisión y la trayectoria son también adecuados para cada solitón.

El comportamiento asintótico lo podemos ver de manera similar al de los solitones de sine-Gordon; Sección 2.8.5. Para esto, escribiendo la expresión (4.4.31) en la forma

$$
\tan \left(\frac{\arcsin \left(\chi^{1 / 2} \psi^{2}\right)}{2}\right)=\mathbb{B} \frac{\sinh \left(\frac{\zeta_{1}-\zeta_{2}}{2}\right)}{\cosh \left(\frac{\zeta_{1}+\zeta_{2}}{2}\right)}
$$

donde utilizaremos la colisión kink-antikink, con condiciones similares a las mencionadas en dicha sección: $m_{1}=m_{2}=m, v_{1}=-v_{2}=v>0$ y $x_{0}=-x_{10}=x_{20}>0$. Esto resultó en

$$
\tan \left(\frac{\arcsin \left(\chi^{1 / 2} \psi^{2}\right)}{2}\right)=\frac{c \sinh \left[\gamma m\left(v t-x_{0}\right)\right]}{v \cosh [\gamma m x]}
$$

para adimensionalizar reescribiendo las coordenadas dividiendo entre la longitud de Compton $\lambda_{\text {Compton }}=h / m c, \bar{x}=x / \lambda_{\text {Compton }} \mathrm{y} \bar{t}=t / \lambda_{\text {Compton }}$, obtenemos

$$
\tan \left(\frac{\arcsin \left(\chi^{1 / 2} \psi^{2}\right)}{2}\right)=\frac{c \sinh \left[\gamma\left(v \bar{t}-\bar{x}_{0}\right)\right]}{v \cosh [\gamma \bar{x}]}
$$


como en aquel caso

$$
\begin{aligned}
& \underset{\bar{x} \rightarrow+\infty}{\stackrel{\bar{t} \rightarrow+\infty}{\longrightarrow}} \mathrm{e}^{\gamma\left(\bar{x}-\bar{x}_{0}+v \bar{t}+\Delta\right)} \\
& \underset{\bar{x} \rightarrow-\infty}{\stackrel{\bar{t} \rightarrow+\infty}{\longrightarrow}} \mathrm{e}^{-\gamma\left(\bar{x}+\bar{x}_{0}-v \bar{t}-\Delta\right)} \\
& \underset{\bar{x} \rightarrow+\infty}{\stackrel{\bar{t} \rightarrow-\infty}{\longrightarrow}}-\mathrm{e}^{-\gamma\left(\bar{x}-\bar{x}_{0}+v \bar{t}-\Delta\right)} \\
& \underset{\bar{x} \rightarrow-\infty}{\stackrel{\bar{t} \rightarrow-\infty}{\longrightarrow}}-\mathrm{e}^{\gamma\left(\bar{x}+\bar{x}_{0}-v \bar{t}+\Delta\right)}
\end{aligned}
$$

donde en cada una de estas cuatro aproximaciones tenemos corrimientos para cada solitón de la forma $\Delta=\gamma^{-1} \ln |\mathbb{B}|=\gamma^{-1} \ln |-c / v|$. Invirtiendo para recuperar $\psi$ tenemos para $\bar{t} \rightarrow+\infty$

$$
\begin{aligned}
& \bar{x} \underset{+\infty}{\longrightarrow} \frac{1}{\chi^{1 / 4}}\left[\sin \left\{2 \arctan \left(\mathrm{e}^{\gamma\left(\bar{x}-\bar{x}_{0}+v \bar{t}+\Delta\right)}\right)\right\}\right]^{1 / 2} \\
& \left.\bar{x} \underset{-\infty}{\longrightarrow} \frac{1}{\chi^{1 / 4}}\left[\sin \left\{2 \arctan \left(\mathrm{e}^{-\gamma\left(\bar{x}+\bar{x}_{0}-v \bar{t}-\Delta\right.}\right)\right)\right\}\right]^{1 / 2}
\end{aligned}
$$

y para $\bar{t} \rightarrow-\infty$

$$
\begin{aligned}
& \left.\bar{x} \underset{+\infty}{\longrightarrow} \frac{1}{\chi^{1 / 4}}\left[\sin \left\{2 \arctan \left(-\mathrm{e}^{-\gamma\left(\bar{x}-\bar{x}_{0}+v \bar{t}-\Delta\right.}\right)\right)\right\}\right]^{1 / 2} \\
& \bar{x} \underset{-\infty}{\longrightarrow} \frac{1}{\chi^{1 / 4}}\left[\sin \left\{2 \arctan \left(-\mathrm{e}^{\gamma\left(\bar{x}+\bar{x}_{0}-v \bar{t}+\Delta\right)}\right)\right\}\right]^{1 / 2}
\end{aligned}
$$

por la construcción del mapeo, estas dos últimas expresiones generan valores complejos, esto lo podemos solucionar, ya sea, multiplicando por $-i$ o sustituyendo la representación del kink y antikink por sus equivalentes en un sector positivo, esto es

$$
\begin{aligned}
& \bar{x} \underset{+\infty}{\longrightarrow} \frac{1}{\chi^{1 / 4}}\left[\sin \left\{2 \arctan \left(\mathrm{e}^{\gamma\left(\bar{x}-\bar{x}_{0}+v \bar{t}-\Delta\right)}\right)\right\}\right]^{1 / 2} \\
& \left.\bar{x} \underset{-\infty}{\longrightarrow} \frac{1}{\chi^{1 / 4}}\left[\sin \left\{2 \arctan \left(\mathrm{e}^{-\gamma\left(\bar{x}+\bar{x}_{0}-v \bar{t}+\Delta\right.}\right)\right)\right\}\right]^{1 / 2}
\end{aligned}
$$

Cada una de las funciones aproximadas asintóticas (4.4.39) y (4.4.41), son solitones de Lane-Emden, cada uno con un corrimiento $\Delta$.

\subsubsection{El corrimiento $\Delta$}

Con el fin de analizar los corrimientos veamos un caso más general, con velocidades, masas y posiciones iniciales distintas. Sólo considerando que inciden en dirección opuesta partiendo de distinta posición, para lo cual en (4.4.35), escribiremos

$$
\begin{aligned}
& \zeta_{1}=\gamma_{1}\left(\tilde{x}_{1}+\tilde{x}_{10}-v_{1} \tilde{t}_{1}\right) \\
& \zeta_{2}=\gamma_{2}\left(\tilde{x}_{2}-\tilde{x}_{20}+v_{2} \tilde{t}_{2}\right)
\end{aligned}
$$


y tenemos que

$$
\begin{aligned}
\zeta_{1} \pm \zeta_{2} & =\gamma_{1} m_{1}\left(x+x_{10}-v_{1} t\right) \pm \gamma_{2} m_{2}\left(x-x_{20}+v_{2} t\right) \\
& =\tilde{\gamma}^{( \pm)}\left[x-\tilde{x}_{0}^{( \pm)}-\tilde{v}^{( \pm)} t\right]
\end{aligned}
$$

donde definimos

$$
\begin{aligned}
\tilde{\gamma}^{( \pm)} & =\gamma_{1} m_{1} \pm \gamma_{2} m_{2} \\
\tilde{v}^{( \pm)} & =\frac{\gamma_{1} v_{1} m_{1} \mp \gamma_{2} v_{2} m_{2}}{\gamma_{1} m_{1} \pm \gamma_{2} m_{2}} \\
\tilde{x}_{0}^{( \pm)} & =-\frac{\gamma_{1} x_{10} m_{1} \mp \gamma_{2} x_{20} m_{2}}{\gamma_{1} m_{1} \pm \gamma_{2} m_{2}}
\end{aligned}
$$

la más importante de estas expresiones es (4.4.44b), de la cual

$$
\tilde{v}^{(+)}=\frac{\gamma_{1} v_{1} m_{1}-\gamma_{2} v_{2} m_{2}}{\gamma_{1} m_{1}+\gamma_{2} m_{2}}
$$

es la velocidad que se obtiene cuando dos lumps colisionan inelásticamente durante el impacto en direcciones opuestas, a la vez que

$$
\tilde{v}^{(-)}=\frac{\gamma_{1} v_{1} m_{1}+\gamma_{2} v_{2} m_{2}}{\gamma_{1} m_{1}-\gamma_{2} m_{2}}
$$

puede entenderse como la separación de las mismas. Cuando las masas $m_{1}=m_{2}$ en las expresiones (4.4.44), podemos escribir

$$
\begin{aligned}
\gamma^{( \pm)} & =\gamma_{1} \pm \gamma_{2} \\
\beta^{( \pm)}=\frac{v^{( \pm)}}{c} & =\frac{\gamma_{1} \beta_{1} \mp \gamma_{2} \beta_{2}}{\gamma_{1} \pm \gamma_{2}}
\end{aligned}
$$

con lo anterior $(2.8 .35)$ se puede escribir como

$$
\mathbb{B}=\frac{a_{1}+a_{2}}{a_{1}-a_{2}}=\frac{a^{(+)}}{a^{(-)}}=\frac{\gamma^{(+)}\left(1-\beta^{(+)}\right)}{\gamma^{(-)}\left(1-\beta^{(-)}\right)} .
$$

La separación asintótica puede realizarse a partir de (4.4.35) tal como en los casos anteriores, lo que resulta en

$$
\begin{aligned}
\varphi_{(\tilde{t}, \tilde{x}) \rightarrow(-\infty,+\infty)} & \approx \frac{1}{\chi^{1 / 4}}\left[\sin \left\{2 \arctan \left(\mathrm{e}^{-\gamma_{2} m_{2}\left(x-x_{20}+v_{2} t\right)}\right)\right\}\right]^{1 / 2} \\
\varphi_{(\tilde{t}, \tilde{x}) \rightarrow(-\infty,-\infty)} & \approx \frac{1}{\chi^{1 / 4}}\left[\sin \left\{2 \arctan \left(\mathrm{e}^{\gamma_{1} m_{1}\left(x+x_{10}-v_{1} t\right)}\right)\right\}\right]^{1 / 2} \\
\varphi_{(\tilde{t}, \tilde{x}) \rightarrow(+\infty,-\infty)} & \approx \frac{1}{\chi^{1 / 4}}\left[\sin \left\{2 \arctan \left(\mathrm{e}^{-\gamma_{2} m_{2}\left(x-x_{20}+2 \Delta_{2}+v_{2} t\right)}\right)\right\}\right]^{1 / 2} \\
\varphi_{(\tilde{t}, \tilde{x}) \rightarrow(+\infty,+\infty)} & \approx \frac{1}{\chi^{1 / 4}}\left[\sin \left\{2 \arctan \left(\mathrm{e}^{\gamma_{1} m_{1}\left(x+x_{10}-2 \Delta_{1}-v_{1} t\right)}\right)\right\}\right]^{1 / 2}
\end{aligned}
$$


En estas últimas expresiones hemos trasladado todo el corrimiento a los solitones en $t \rightarrow+\infty$, y cada corrimiento tiene la forma $\Delta_{i}=\left(m_{i} \gamma_{i}\right)^{-1} \ln |\mathbb{B}|$.

En cada uno de los solitones asintóticos obtenidos, los corrimientos pueden escribirse usando (4.4.48) como

$$
\Delta_{i}=\left(m_{i} \gamma_{i}\right)^{-1} \ln |\mathbb{B}|=\left(m_{i} \gamma_{i}\right)^{-1} \ln \left|\frac{\gamma^{(+)}\left(1-\beta^{(+)}\right)}{\gamma^{(-)}\left(1-\beta^{(-)}\right)}\right|
$$

además utilizando (2.7.21), notamos que se pueden separar dos corrimientos

$$
\Delta_{i}=\left(m_{i} \gamma_{i}\right)^{-1} \ln \left|\frac{a^{(+)}}{a^{(-)}}\right|=\left(m_{i} \gamma_{i}\right)^{-1}\left(\ln \left|a^{(+)}\right|-\ln \left|a^{(-)}\right|\right)
$$

es decir, podemos separar de la forma

$$
\Delta_{i}=\Delta_{i}^{(+)}-\Delta_{i}^{(-)}
$$

aquí el primero $\Delta_{i}^{(+)}$resultado de

$$
a^{(+)}=\gamma^{(+)}\left(1-\beta^{(+)}\right)
$$

notemos que éste tiene la forma de efecto Doppler relativista, más aún puede entender como resultado de la unión de los solitones. Mientras que el segundo $\Delta_{i}^{(-)}$debido a

$$
a^{(-)}=\gamma^{(-)}\left(1-\beta^{(-)}\right)
$$

se puede entender por la separación de los mismos.

En las expresiones (4.4.49), tenemos para las coordenadas del solitón identificado por 1

$$
\gamma_{1} m_{1}\left(x-2 \Delta_{1}-v_{1} t+x_{10}\right)=\gamma_{1} m_{1}\left(\left[x-2 \Delta_{1}^{(+)}\right]+x_{10}-v_{1}\left[t-2 \frac{\Delta_{1}^{(-)}}{v_{1}}\right]\right)
$$

y para el identificado por 2, queda

$$
-\gamma_{2} m_{2}\left(x+2 \Delta_{2}+v_{2} t-x_{20}\right)=-\gamma_{2} m_{2}\left(\left[x+2 \Delta_{2}^{(+)}\right]-x_{20}+v_{2}\left[t-2 \frac{\Delta_{2}^{(-)}}{v_{2}}\right]\right)
$$

al escribir esto, podemos justificar de una mejor manera que la unión de los solitones tiene una consecuencia espacial, una separación; mientras que la separación una temporal, es un retraso. 



\section{Capítulo 5}

\section{Solitones de Klein-Gordon en $(2+1) \mathrm{D}$}

Si bien una gran cantidad de conceptos teóricos se pueden expresar de manera adecuada sin restringir la cantidad de dimensiones espaciales, la realidad ha mostrado que la mayor cantidad de modelos exactamente solubles está dada únicamente en 1 dimensión espacial y 1 temporal; es decir $(1+1)$ D. Es por ello que la extensión de ecuaciones no lineales de $1+1$ dimensiones a modelos con más dimensiones espaciales, es decir, modelos $(2+1) \mathrm{D}$ o $(3+1) \mathrm{D}$, puede ser muy variada y en general más difícil. Algunas extensiones pueden ser muy naturales, únicamente considerando la variable dependiente con más grados de libertad y las consecuentes extensiones de los operadores involucrados, esto principalmente se da en casos simétricos (isotrópicos). Mientras que otras, por el contrario podrían llevar a un replanteamiento completo de las ecuaciones que describen el sistema; rompimientos de simetría. Esta situación lleva también a la posible obtención de distintas extensiones de la misma ecuación según la aplicación buscada del modelo. Una consecuencia directa de tener más grados de libertad, es que los métodos utilizados en $(1+1) \mathrm{D}$ en general no garantizan que la integrabilidad del sistema se mantenga.

En este capítulo, primeramente exponemos utilizando el modelo de sine-Gordon cómo una extensión a $2+1$ dimensiones, genera una familia de soluciones, no del todo definida, aunque exacta. Posteriormente de manera análoga, utilizamos el modelo de Lane-Emden para extender las solución planteada, y mostrar algunos casos de utilidad para este modelo de juguete.

\subsection{Modelo sine-Gordon (2+1)D: Uniones de Josephson}

Entre las muchas aplicaciones del modelo sine-Gordon, algunas se encuentran en óptica cuántica no lineal, en la descripción de las uniones de Josephson en fenómenos de 
superconductividad, así como en superconductores a alta temperatura.

Las uniones de Josephson son dispositivos construidos a partir del empalmado de una capa delgada uniforme de material no superconductor entre dos capas de material superconductor. En 1962, Brian D. Josephson predijo que pares de electrones superconductores, hoy llamados pares de Cooper, podrían tunelar la capa no superconductora como en la mecánica cuántica. Predijo además, la forma exacta de la relación entre la corriente y el voltaje para la unión. Estas predicciones fueron probadas experimentalmente y posteriormente le merecieron el premio Nobel en 1973.

La dinámica de estos dispositivos es aproximadamente descrita por el modelo de sineGordon perturbado; vid. Lomdahl (1985). Así, en el caso de la geometría planteada anteriormente, la ecuación $2+1$ dimensional para la diferencia entre las fases de los parámetros de orden de los dos conductores $\varphi=\varphi(t, x, y)$ es

$$
\varphi_{x x}+\varphi_{y y}=\sin \varphi+\frac{1}{c_{\mathrm{eff}}^{2}} \varphi_{t t}+\alpha \varphi_{t}
$$

donde $\alpha=(\hbar / 2 e R) \omega_{p}$, con $\omega_{p}$ la frecuencia del plasma, $R$ la resistencia normal efectiva, $e$ la carga del electrón, $\hbar$ la constante de Planck reducida y $c_{\text {eff }}$ la velocidad efectiva en el plasma. Considerando una separación de variables $\varphi(t, x, y)=\varphi_{1}(t, x)+\varphi_{2}(y)$, la ecuación (5.1.1) se reduce a la ecuación de sine-Gordon perturbada en una dimensión

$$
\varphi_{1, x x}=\sin \varphi_{1}+\alpha \varphi_{1, t}-\eta+\varphi_{1, t t}
$$

donde $\eta$ podría depender de $x$, es decir, es una ecuación amortiguada-forzada de sineGordon. Para que esto cumpla la función

$$
\varphi_{2}(y)=\frac{\eta y^{2}}{2}, \quad \frac{\eta}{2}\left(\frac{W}{2}\right)^{2} \ll 1
$$

en ésta $W$ es la longitud de empalme en dirección de la corriente. Resolviendo la ecuación perturbada sin los términos de amortiguamiento y forzamiento proporciona la soluciones kink/antikink como una buena aproximación del fenómeno. Un hecho es que estos términos no afectan la existencia del solitón; vid. Eilbeck et al. (1985); Ustinov (1998).

El solitón o kink en la unión de Josephson representa un salto de $2 \pi$ en la diferencia de fase $(\varphi)$ a través de la barrera no superconductora que separa los dos superconductores. En otras palabras, la corriente forma el circuito conectando las dos superficies a través de la barrera. Este solitón es llamado fluxón, ya que el salto encierra un cuanto de de flujo magnético $\Phi_{0}=h / 2 e=2.07 \times 10^{-15} \mathrm{~Wb}$; también son llamados vórtices de Josephson, Figura 5.1.1. 


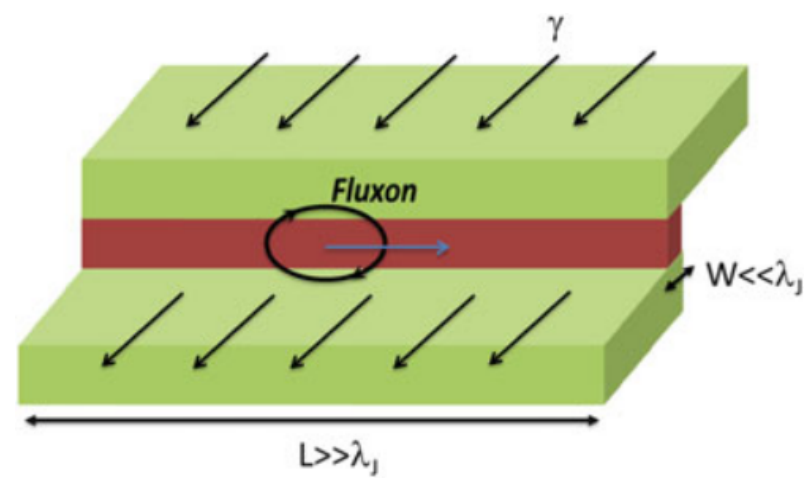

Figura 5.1.1: Esquema de la unión de Josephson.

\subsubsection{Solitón simple en $(2+1) \mathrm{D}$}

Una descripción más realista del sistema físico implica resolver la ecuación (5.1.1); sin embargo, la ecuación en dos dimensiones espaciales, inclusive sin el término de amortiguamiento no es totalmente integrable. Por esto, es más conveniente partir de la ecuación en una dimensión espacial y ésta extenderla al sistema de dos dimensiones espaciales. Cada extensión del proceso puede realizarse con distintos objetivos, los que pueden conducir a diferentes extensiones.

Para este caso, la ecuación (5.1.1) describe la propagación de un fluxón, de forma arbitraria a lo largo de la línea que une los superconductores (línea de vórtice). Esto puede ser útil para transferir información en varios dispositivos. Una posible aplicación es la introducción de un reloj sobre el fluxón.

Para este proceso consideraremos la ecuación (5.1.1) sin el término de amortiguamiento, esto es

$$
\varphi_{x x}+\varphi_{y y}-\frac{1}{c^{2}} \varphi_{t t}=\sin \varphi
$$

Estas simplificación del sistema original nos permite proponer una solución, tal como Gulevich et al. (2008, 2009),

$$
\tan \left(\frac{\varphi}{4}\right)=\mathrm{e}^{y} \mathrm{e}^{-f(x, t)}
$$

Esta proposición corresponde a la solución kink (2.8.15) para $y$ cuando $f(x, t)$ es trivial. Al considerar una función de la forma $f(x \pm u t)$, la solución $\varphi$ puede escribirse como

$$
\varphi(x, y, t)=4 \arctan \{\exp [y-f(x \pm v t)]\}
$$

escribiendo la fase $s=x \pm u t$ y calculando las segundas derivadas tenemos

$$
\varphi_{t t}=-2 u^{2} \operatorname{sech}(y-f(s))\left[\tanh (y-f(s))\left(\frac{\partial f}{\partial s}\right)^{2}+\frac{\partial^{2} f}{\partial s^{2}}\right]
$$




$$
\begin{gathered}
\varphi_{x x}=-2 \operatorname{sech}(y-f(s))\left[\tanh (y-f(s))\left(\frac{\partial f}{\partial s}\right)^{2}+\frac{\partial^{2} f}{\partial s^{2}}\right] \\
\varphi_{y y}=-2 \operatorname{sech}(y-f(s)) \tanh (y-f(s))
\end{gathered}
$$

sustituyendo en la ecuación (5.1.4), ésta se reduce

$$
0=2\left(\frac{v^{2}}{c^{2}}-1\right) \operatorname{sech}(y-f(s))\left[\tanh (y-f(s))\left(\frac{\partial f}{\partial s}\right)^{2}+\frac{\partial^{2} f}{\partial s^{2}}\right]
$$

con lo que, se comprueba la que la solución propuesta es solución para los casos límite siguientes:

1. Asintótico: si $y-f(s) \rightarrow \pm \infty$,

2. Ideal: si $v= \pm c$,

En estos casos, la función $f(x \pm v t)$ real-valuada, diferenciable dos veces, arbitraria y convenientemente localizada satisface de manera exacta la ecuación de $(2+1) \mathrm{D}$ de sineGordon, y representa excitaciones asociadas con el corrimiento local. Se presenta además, un tercer caso que la función debe satisface,

$$
\tanh (y-f(s))\left(\frac{\partial f}{\partial s}\right)^{2}+\frac{\partial^{2} f}{\partial s^{2}}=0
$$

La solución que hemos obtenido es estacionaria, por lo que para la introducción del tiempo, es necesario la aplicación de un boost de Lorentz, debido a que la ecuación (5.1.4) es invariante ante transformaciones de Lorentz. Si aplicamos la transformación a lo largo de la dirección $y$ las variables se transforman en

$$
\begin{aligned}
x & \rightarrow x^{\prime}=x \\
y & \rightarrow y^{\prime}=\gamma(y-c \beta t) \\
c t & \rightarrow c t^{\prime}=\gamma(c t-\beta y)
\end{aligned}
$$

donde el factor de Lorentz $\gamma=\left(1-\beta^{2}\right)^{-1 / 2}$ y $\beta=v / c$; con lo que la solución en el tiempo se puede ver como

$$
\phi(t, x, y)=4 \arctan \left\{\exp \left[\frac{y-c \beta t}{\sqrt{1-\beta^{2}}}-f\left(x \pm \beta \frac{c t-\beta y}{\sqrt{1-\beta^{2}}}\right)\right]\right\}
$$

\subsection{Soluciones de Lane-Emden en $(2+1) \mathrm{D}$}

Con la intención de trasladar los resultados parciales obtenidos a soluciones $2+1$ dimensionales a la teoría de halos de materia oscura, consideramos un procedimiento en la 
misma dirección que el planteado en la sección anterior para las uniones de Josephson. Como un primer acercamiento a la extensión del modelo, para buscar soluciones múltiples obtenemos soluciones en $2+1$ dimensiones localizadas.

Introduciendo el potencial de Lane-Emden y un campo de la forma $\phi(t, x, y)$ la ecuación de Klein-Gordon no lineal la podemos escribir como

$$
\left(\frac{\partial^{2}}{\partial x^{2}}+\frac{\partial^{2}}{\partial y^{2}}-\frac{1}{c^{2}} \frac{\partial^{2}}{\partial t^{2}}\right) \phi=m^{2} \phi\left(1-3 \chi \phi^{4}\right) .
$$

Consideremos ahora un reescalamiento con la masa de las coordenadas $\tilde{x}=m x, \tilde{y}=m y$ y $\tilde{t}=m t$, con esto la ecuación (5.2.1) se puede reescribir como

$$
\left(\frac{\partial^{2}}{\partial \tilde{x}^{2}}+\frac{\partial^{2}}{\partial \tilde{y}^{2}}-\frac{1}{c^{2}} \frac{\partial^{2}}{\partial \tilde{t}^{2}}\right) \phi=\phi\left(1-3 \chi \phi^{4}\right)
$$

la cual corresponde a una ecuación de Klein-Gordon no lineal para varias dimensiones como

$$
\tilde{\square} \phi=\phi\left(1-3 \chi \phi^{4}\right)
$$

donde esta ecuación es perfectamente simétrica al intercambio de $x \leftrightarrow y$, y extensible a $3+1$ dimensiones a través del operador D’Alembertiano. Para esta situación, la ecuación conduce a la densidad de energía

$$
\rho(\tilde{t}, \tilde{x}, \tilde{y})=\frac{1}{2}\left[\left(\frac{\partial \phi}{\partial \tilde{x}}\right)^{2}+\left(\frac{\partial \phi}{\partial \tilde{y}}\right)^{2}+\left(\frac{1}{c} \frac{\partial \phi}{\partial \tilde{t}}\right)^{2}\right]+V(\phi),
$$

también simétrica al intercambio de las coordenadas espaciales.

\subsubsection{Solitón simple: caso I}

Recordemos la solución en $1+1$ dimensiones,

$$
\psi(\zeta)= \pm \frac{1}{\chi^{1 / 4}} \sqrt{\operatorname{sech}(2 \zeta)}
$$

donde $\zeta=\gamma(\tilde{x}-v \tilde{t})$, la cual, contiene la dependencia de las variables. De manera general reemplazaremos $\zeta \rightarrow \tilde{y}-f(\tilde{t}, \tilde{x})$, con lo que propondremos una solución de la forma

$$
\psi(\tilde{t}, \tilde{x}, \tilde{y})= \pm \frac{1}{\chi^{1 / 4}} \sqrt{\operatorname{sech}[2(\tilde{y}-f(\tilde{t}, \tilde{x}))]} .
$$

Considerando esta expresión, los términos involucrados en (5.2.3) pueden escribirse como

$$
\begin{aligned}
\frac{\partial^{2}}{\partial \tilde{x}^{2}} \psi(\tilde{t}, \tilde{x}, \tilde{y}) & =\frac{1}{\chi^{1 / 4}} \operatorname{sech}^{5 / 2}\left(\frac{\mathfrak{B}}{2}\right)\left[\left(\frac{\partial f}{\partial \tilde{x}}\right)^{2} \mathfrak{A} \pm \frac{\sinh (\mathfrak{B})}{2}\left(\frac{\partial^{2} f}{\partial \tilde{x}^{2}}\right)\right] \\
\frac{\partial^{2}}{\partial \tilde{t}^{2}} \psi(\tilde{t}, \tilde{x}, \tilde{y}) & =\frac{1}{\chi^{1 / 4}} \operatorname{sech}^{5 / 2}\left(\frac{\mathfrak{B}}{2}\right)\left[\left(\frac{\partial f}{\partial \tilde{t}}\right)^{2} \mathfrak{A} \pm \frac{\sinh (\mathfrak{B})}{2}\left(\frac{\partial^{2} f}{\partial \tilde{t}^{2}}\right)\right]
\end{aligned}
$$




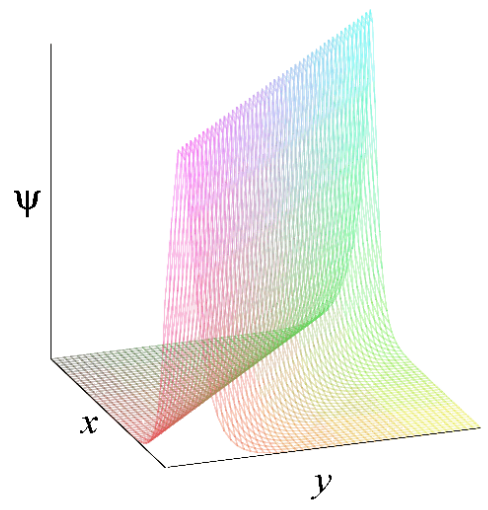

Figura 5.2.1: Solitón $2+1$ dimensional de la forma $\psi=\chi^{-1 / 4} \sqrt{\operatorname{sech}[2(\tilde{y}+\tilde{x}-c \tilde{t})]}$.

$$
\begin{aligned}
\frac{\partial^{2}}{\partial \tilde{y}^{2}} \psi(\tilde{t}, \tilde{x}, \tilde{y}) & =\frac{\mathfrak{A}}{\chi^{1 / 4}} \operatorname{sech}^{5 / 2}\left(\frac{\mathfrak{B}}{2}\right), \\
\psi\left(1-3 \chi \psi^{4}\right) & =\frac{\mathfrak{A}}{\chi^{1 / 4}} \operatorname{sech}^{5 / 2}\left(\frac{\mathfrak{B}}{2}\right),
\end{aligned}
$$

donde escribimos $\mathfrak{B}=4 \tilde{y}-4 f(\tilde{t}, \tilde{x})$ y $\mathfrak{A}= \pm[\cos (\mathfrak{B})-5] / 2$; estas expresiones satisfacen la ecuación (5.2.3) para funciones $f(\tilde{t}, \tilde{x})$ que cubran las condiciones

$$
\begin{aligned}
\frac{1}{c^{2}} \frac{\partial^{2} f}{\partial \tilde{t}^{2}} & =\frac{\partial^{2} f}{\partial \tilde{x}^{2}} \\
\left(\frac{1}{c} \frac{\partial f}{\partial \tilde{t}}\right)^{2} & =\left(\frac{\partial f}{\partial \tilde{x}}\right)^{2} .
\end{aligned}
$$

Estas condiciones se satisfacen para entre otros casos; por ejemplo para funciones de la forma $f(\tilde{t}, \tilde{x})=f(\tilde{x} \pm \tilde{t})$. En este caso para mantener el intercambio de $\tilde{x} \leftrightarrow \tilde{y}$ en la solución, la opción más simple es considerar $f(\tilde{t}, \tilde{x})=-(\tilde{x}-c \tilde{t})$, con lo que la solución toma la forma (Figura 5.2.1)

$$
\psi(\tilde{t}, \tilde{x}, \tilde{y})= \pm \frac{1}{\chi^{1 / 4}} \sqrt{\operatorname{sech}[2(\tilde{y}+\tilde{x}-c \tilde{t})]},
$$

sin embargo; esta elección puede interpretarse como una simple rotación de $\frac{\pi}{2}$ del plano- $\tilde{x} \tilde{y}$, $\tilde{Z}=\tilde{x}+\tilde{y}$ lo que resulta en

$$
\psi(\tilde{t}, \tilde{Z})= \pm \frac{1}{\chi^{1 / 4}} \sqrt{\operatorname{sech}[2(\tilde{Z}-c \tilde{t})]} .
$$

Elegir una solución más complicada pero localizada en $(1+2) \mathrm{D}$, puede darse tomando una función localizada, por ejemplo $f(\tilde{t}, \tilde{x})=k \operatorname{sech}(\tilde{x}-c \tilde{t})$ resulta en una solución de la forma

$$
\psi(\tilde{t}, \tilde{x}, \tilde{y})= \pm \frac{1}{\chi^{1 / 4}} \sqrt{\operatorname{sech}[2(\tilde{y}-k \operatorname{sech}(c \tilde{t}-\tilde{x}))]}
$$




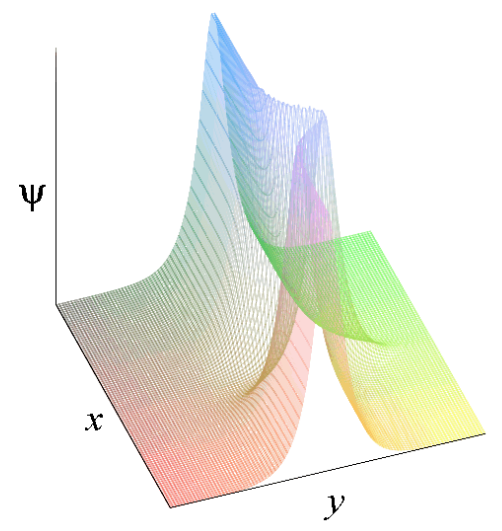

Figura 5.2.2: Solitón $2+1$ dimensional de la forma $\psi=\chi^{-1 / 4} \sqrt{\operatorname{sech}[2(\tilde{y}-k \operatorname{sech}(c \tilde{t}-\tilde{x}))}$.

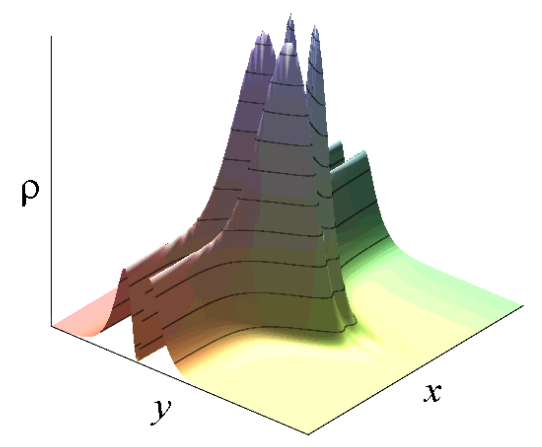

Figura 5.2.3: Densidad de energía $\rho$, para $\psi=\chi^{-1 / 4} \sqrt{\operatorname{sech}[2(\tilde{y}-k \operatorname{sech}(c \tilde{t}-\tilde{x}))]}$.

mostrada en la Figura 5.2.2, donde hemos escrito $\zeta=c \tilde{t}-\tilde{x}$. A la vez, la densidad de energía la obtenemos utilizando la ecuación (5.2.4) y obtenemos (Figura 5.2.3)

$$
\begin{gathered}
\rho(\tilde{t}, \tilde{x}, \tilde{y})=\frac{m^{2}}{\sqrt{\chi}}\left(\operatorname{sech}^{3}[\mathfrak{D}]-\operatorname{sech}[\mathfrak{D}]\right)\left\{k^{2} \gamma^{2}\left(\operatorname{sech}^{4} \zeta-\operatorname{sech}^{2} \zeta\right)-1\right\} \\
\mathfrak{D}=\frac{2 \tilde{y} \cosh \zeta-k}{\cosh \zeta}
\end{gathered}
$$

integrando esto, la energía del solitón resulta en

$$
E=\int_{x=-L}^{x=+L} \int_{y=-\infty}^{y=+\infty} \rho(t, x, y) d y d x=\frac{\pi}{\sqrt{\chi}}(2 L+k)
$$

donde $L$ es el largo de la región donde consideramos el solitón. 


\subsubsection{Solitón simple: caso II}

Buscando localizar el solitón en ambas dimensiones espaciales, propongamos una solución e la forma

$$
\psi(\tilde{t}, \tilde{x}, \tilde{y})= \pm \frac{1}{\chi^{1 / 4}} \sqrt{\operatorname{sech}(2 \tilde{y}) \operatorname{sech}(k f(\tilde{t}, \tilde{x}))} .
$$

Sustituyendo en la ecuación (5.2.2), las derivadas y el término independiente resultan en

$$
\begin{gathered}
\frac{\partial^{2}}{\partial \tilde{x}^{2}} \psi=-\psi\left[\left(\frac{k}{2}\right)^{2}\left(3 \operatorname{sech}^{2}(k f)-1\right)\left(\frac{\partial f}{\partial \tilde{x}}\right)^{2}+\frac{k}{2} \tanh (k f)\left(\frac{\partial^{2} f}{\partial \tilde{x}^{2}}\right)\right](5 \\
\frac{\partial^{2}}{\partial \tilde{t}^{2}} \psi=+\psi\left[\left(\frac{k}{2}\right)^{2}\left(3 \operatorname{sech}^{2}(k f)-1\right)\left(\frac{\partial f}{\partial \tilde{t}}\right)^{2}+\frac{k}{2} \tanh (k f)\left(\frac{\partial^{2} f}{\partial \tilde{t}^{2}}\right)\right](5 \\
\frac{\partial^{2}}{\partial \tilde{y}^{2}} \psi=-\psi\left[3 \operatorname{sech}^{2}(2 \tilde{y})-1\right] \\
\psi\left(1-3 \chi \psi^{4}\right)=-\psi\left[3 \operatorname{sech}^{2}(2 \tilde{y}) \operatorname{sech}^{2}(k f)-1\right]
\end{gathered}
$$

Esta propuesta de solución con las condiciones (5.2.8), nos conduce a que la ecuación (5.2.1) se escriba como

$$
\operatorname{sech}^{2}(2 \tilde{y})\left[\operatorname{sech}^{2}(k f(\tilde{t}, \tilde{x}))-1\right]=0
$$

la cual sólo es exacta para $\tilde{y} \rightarrow \pm \infty$ o en el caso que $f(\tilde{t}, \tilde{x}) \rightarrow 0$.

Por otra parte, la densidad de energía resulta en

$$
\begin{aligned}
& \rho(\tilde{x}, \tilde{y}, t)=\frac{m^{2}}{\sqrt{\chi}} \operatorname{sech}(k f) \operatorname{sech}(p \tilde{y})\left[1-\frac{1}{2} \operatorname{sech}^{2}(2 \tilde{y})\left\{1+\operatorname{sech}^{2}(k f)\right\}\right. \\
&\left.+\frac{k^{2}}{8}\left(\tanh ^{2}(k f)\right)\left\{\left(\frac{\partial f}{\partial \tilde{x}}\right)^{2}+\left(\frac{1}{c} \frac{\partial f}{\partial \tilde{t}}\right)^{2}\right\}\right]
\end{aligned}
$$

eligiendo la función $f(\tilde{t}, \tilde{x})=\tilde{x}-c \tilde{t}$ la solución propuesta resulta (figura 5.2.4)

$$
\psi=\frac{1}{\chi^{1 / 4}} \sqrt{\operatorname{sech}(2 \tilde{y}) \operatorname{sech}(k(\tilde{x}-c \tilde{t}))}
$$

y la densidad densidad de energía (figura 5.2.5)

$$
\begin{gathered}
\rho(\tilde{x}, \tilde{y}, t)=\frac{m^{2}}{\sqrt{\chi}} \operatorname{sech}(k(x-c t)) \operatorname{sech}(p \tilde{y})\left[1-\frac{1}{2} \operatorname{sech}^{2}(2 \tilde{y})\left\{1+\operatorname{sech}^{2}(k(x-c t))\right\}\right. \\
\left.+\frac{k^{2}}{4} \tanh ^{2}(k(x-c t))\right]
\end{gathered}
$$

Debemos recordar que las extensiones de los modelos se realizan la mayoría de veces con fines particulares. Estos ejemplos no han incluido simetrías más especificas como 


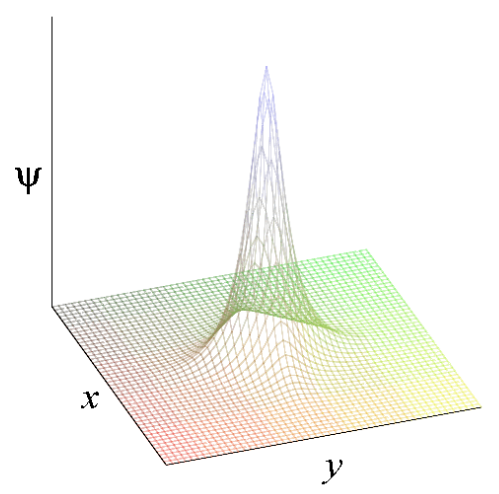

Figura 5.2.4: Solitón $2+1$ dimensional de la forma $\psi=\chi^{-1 / 4} \sqrt{\operatorname{sech}(2 \tilde{y}) \operatorname{sech}(k(\tilde{x}-c \tilde{t}))}$

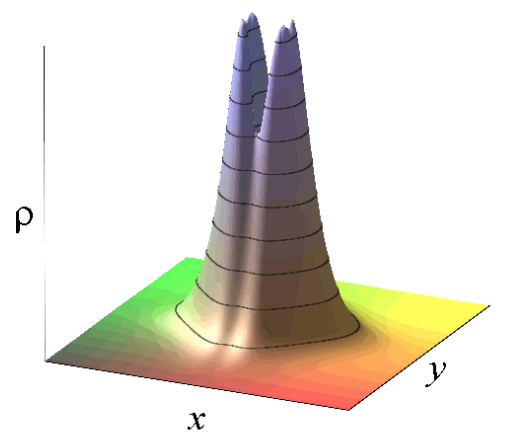

Figura 5.2.5: Densidad de energía $\rho$, para $\psi=\chi^{-1 / 4} \sqrt{\operatorname{sech}(2 \tilde{y}) \operatorname{sech}(k(\tilde{x}-c \tilde{t}))}$

puede ser el caso axisimétrico o el torodidal, que imponen constricciones y rangos para las coordenadas. Utilizar éstas, permitiría ya sea soluciones exactas o aproximaciones mejores, a la vez que permitirían plantear algún escenario para explorar las colisiones de los mismos. Es importante aclarar que el hecho de que no exista una transformación de Bäcklund exacta en $1+1$ dimensiones (ec. (2.7.2)) no impone ninguna restricción para el caso $2+1$ o de mayor dimensionalidad. 



\section{Capítulo 6}

\section{Conclusiones y expectativas}

Nuestros actuales métodos analíticos parecen inadecuados para la solución de los problemas importantes que surgen de la relación con las ecuaciones diferenciales parciales no lineales $y$, de hecho, con prácticamente todos los tipos de problemas no lineales en matemática pura.

John Von Neumann

En los dos capítulos anteriores hemos expuestos la versión matemática de los resultados obtenidos y el punto de vista propuesto para aproximar las soluciones, en el presente exponemos el punto de vista físico, respecto a las posibles consecuencias, de las cuales algunas fueron mencionadas en su momento aunque otras se han pospuesto a este capítulo.

\subsection{Conclusiones}

Sabemos que el proceso de construcciones solitónicas múltiples no es una tarea automatizada y, en la mayoría de casos, ésta está ligada a las constricciones físicas planteadas por el sistema o por los métodos empleados. También es importante notar que en muchas ocasiones puede resultar de mayor utilidad una aproximación sistemática simple que una solución particular exacta y compleja. Este planteamiento toma mayor importancia en la teoría no lineal, ya que cada ecuación o variante de la misma puede conducir a familias completas de problemas, o ayudar a contextualizar semejanzas entre fenómenos aparentemente no relacionados. Así como la extensión dimensional puede abrir nuevos caminos para la existencia de fenómenos ausentes en modelos $1+1$ dimensionales.

La situación planteada por el potencial de Lane-Emden como un potencial localizado, en comparación al potencial periódico de sine-Gordon resulta de gran utilidad al pensar 
en fenómenos de los que carecemos de información para períodos muy extensos, como es el caso de las colisiones galácticas, ya que en eventos de duración temporal tan extendida pueden considerarse localizados.

Si bien en el caso de solitones múltiples hemos utilizado una aproximación y no la solución exacta, debemos hacer énfasis en que asintóticamente se recuperan solitones como los esperados, esto es importante ya que las propiedades para un solitón es lo que establecen. Aunado a esto, él que la teoría no permita la autotransformación exacta respalda el uso de la aproximación. Otros métodos como el de Hirota podrían brindar una alternativa a tal situación; vid. C.

En escenarios astrofísicos, la longitud de Compton de las partículas de materia oscura en los halos de galaxias es del orden del tamaño de la misma, es decir, $\lambda_{\text {Compton }}=h / m c \sim$ $10 \mathrm{kpc}$. Esta estimación, conduce a que partículas ultraligeras de masa $m \simeq 10^{-26} \mathrm{eV}$, un valor 20 órdenes de magnitud por debajo de usual predicho para la masa de los axiones invisibles $m_{a} \simeq 10^{-6} \mathrm{eV}$, pero superior a la escala de Hubble de $10^{-31} \mathrm{eV}$. Otro escenario en la predicción de este valor, es que en una primera etapa los axiones invisibles de $\mu \mathrm{eV}$ colapsen a mini-MACHOS, los que después formen el halo vía grandes nubes de materia oscura; vid. Mielke y Schunck (2001).

El posible comportamiento tipo solitón podría explicar el peculiar comportamiento que se presenta en algunos cúmulos galácticos como el Abell 520 y el Bullet, ambos sin una solución clara en el modelo estándar de materia oscura CDM. En el Abell 520, los retrasos que presenta el núcleo de materia oscura podría deberse a la colisión (en progreso) de dos o más solitones Lane-Emden. Mientras que para el Bullet, que es más antiguo, la bala podría deberse a que la colisión ya ha tenido lugar y los solitones se están alejando. En ambos casos, considerar que los corrimientos $\Delta$ presentes durante la colisión tienen efectos en las velocidades y estructura de cada solitón; podrían ser una alternativa al acoplamiento entre energía oscura y materia oscura; vid. Lee y Baldi (2011). Para los cúmulos mencionados, se estima que las velocidades de colisión son: para el Abell 520 de $4700 \mathrm{~km} / \mathrm{s}$ y para el Bullet de $1066 \mathrm{~km} / \mathrm{s}$, respectivamente; vid. Dawson et al. (2012); resultando en corrimientos adimensionales de -11.4 y -8.3 , respectivamente; Castañeda Valle y Mielke (2014).

En $2+1$ dimensiones los resultados por ahora son parciales y existen inconvenientes respecto a la exactitud y respecto al intercambio de coordenadas. Estas se han buscado en la dirección de ser aplicables a la colisión de halos galácticos, y los comportamientos esperados son satisfactorios. El resultado podría ser de utilidad para analizar regiones o inclusive ciertas nubes de materia oscura. 


\subsection{Expectativas y trabajo futuro}

Las aproximaciones logradas han sido de utilidad para estimar cualitativamente el comportamiento de la colisión de solitones de Lane-Emden, sin embargo; para lograr una mejor aproximación al problema real es necesario considerar el problema completo, es decir, 3+1 dimensional. De hecho, es necesario considerar no sólo la ecuación de Klein-Gordon, también las ecuaciones de Einstein como el sistema acoplado.

Por otra parte, considerar geometrías diferentes o situaciones de colisión más específicas como un caso axisimétrico, podría proporcionar constantes de movimiento y aclarar el grado de integrabilidad del modelo. Estudiar la estabilidad también es importante, ya que por ahora, ésta se está atribuyendo a la trampa gravitacional en la que se encuentra el solitón, pero ésta podría verse afectada por los mismos corrimientos, es decir, por las masas y velocidades de los objetos involucrados en la colisión.

La complejidad de los Ansätze planteados en el análisis $(2+1) \mathrm{D}$, sugiere por sí misma la aplicación de una transformación que permita simplificar el desarrollo al buscar soluciones múltiples. La elección de la transformación misma está asociada al método de Hirota.

Si bien aproximar soluciones es de gran utilidad para el análisis cualitativo, realizar la comparación con soluciones numéricas se ha convertido en un gran método de validación, ya que en la actualidad existen muchas herramientas para simular escenarios que incluyen datos obtenidos de mediciones; por ejemplo, comparaciones en distintos escenarios como lo realizado en Lee y Baldi (2011) al utilizar los datos de WMAP7. En esta dirección, obtener soluciones numéricas podría ser de gran utilidad.

Una situación aún más ambiciosa sería considerar solitones vectoriales, los que podrían de una mejor manera explicar el comportamiento del núcleo del Abell 520, al considerar modos internos para los distintos nucleos. 



\section{Bibliografía}

Alagesan, T., Chung, Y., Y Nakkenran, K. Soliton Solutions of Coupled Nonlinear Klein-Gordon Equations. Chaos, Solitons \& Fractals 21(4):879-882 (2004)

Avelar, A.T., Bazeia, D., Losano, L., Y Menezes, R. New Lump-Like Structures in Scalar-Field Models. The European Physical Journal C 55:133-143 (2007)

Avelar, A.T., Bazeia, D., Cardoso, W.B., y Losano, L. Lump-Like Structures in Scalar-Field Models in Dimensions. Physics Letters A 374(2):222-227 (2009)

BAlDi, M. Multiple Dark Matter as a Self-Regulating Mechanism for Dark Sector Interactions. Annalen der Physik 524(9-10):602-617 (2012)

Baltz, E.A. Dark Matter Candidates. En SLAC Summer Institute on Particle Physics (2004)

Barone, A., Esposito, F., Magee, C.J., Y Scott, A.C. Theory and Applications of the sine-Gordon Equation. La Rivista del Nuovo Cimento 1(2):227-267 (1971)

Bernal, A. y Guzmán, F.S. Scalar Field Dark Matter: Head-on Interaction Between Two Structures. Physical Review D 74(10):103002 (2006)

Boulbitch, A. Crystallization of Proteins Accompanied by Formation of a Cylindrical Surface. Physical Review E 56(3):3395-3400 (1997)

Bradač, M., Allen, S.W., Treu, T., Ebeling, H., Massey, R., Morris, R.G., VOn Der Linden, A., Y Applegate, D. Revealing the Properties of Dark Matter in the Merging Cluster MACS J0025.4-1222. The Astrophysical Journal 687(2):959-967 (2008)

Brownstein, J.R. Y Moffat, J.W. The Bullet Cluster 1E0657-558 Evidence Shows Modified Gravity in the Absence of Dark Mmatter. Monthly Notices of the Royal Astronomical Society 382(1):29-47 (2007). 0702146 
Carmeli, M. Group Theory and General Relativity. Imperial College Press (1977)

Carretero-González, R., Frantzeskakis, D.J., Y Kevrekidis, P.G. Nonlinear Waves in Bose-Einstein Condensates: Physical Relevance and Mathematical Techniques. Nonlinearity 21(7):R139-R202 (2008)

Castañeda Valle, D. Y Mielke, E.W. Solitonic Axion Condensates Modeling Dark Matter Halos. Annals of Physics 336:245-260 (2013)

Castañeda Valle, D. Y Mielke, E.W. Increased Infall Velocities in Galaxy Clusters from Solitonic Collisions? Physical Review D 89(4):043504 (2014)

Chandrasekhar, S. An Introduction to the Study of Stellar Structure. Dover, New York (1958)

Cheng, T.P. Y Li, L.F. Gauge Theory of Elementary Particle Physics. Oxford University Press (1983)

Choi, D.I. Collision of Gravitationally Bound Bose-Einstein Condensates. Physical Review A 66(6):63609 (2002)

Clowe, D., Randall, S.W., y Markevitch, M. Catching a Bullet: Direct Evidence for the Existence of Dark Matter. Nuclear Physics B - Proceedings Supplements 173:28$31(2007)$

Coleman, S.R. Classical Lumps and their Quantum Descendants in Aspects of Symmetry: Selected Erice Lectures. Cambridge University Press, Cambridge (1985)

Corbelli, E. Y SAlucci, P. The Extended Rotation Curve and the Dark Matter Halo of M33. Monthly Notices of the Royal Astronomical Society 311:441-447 (2000)

Cottingham, W.N. y Greenwood, D.A. An Introduction to the Standart Model of Particle Physics. 2aㅡ edición. Cambridge University Press (2007)

Dawson, W.A., Wittman, D., Jee, M.J., Gee, P., Hughes, J.P., Tyson, J.A., Schmidt, S., Thorman, P., Bradač, M., Miyazaki, S., Lemaux, B., Utsumi, Y., Margoniner, V.E., Y Vera, E. Discovery of a Dissociative Galaxy Cluster Merger With Large Physical Separation. The Astrophysical Journal 747(2):L42 (2012). arXiv:1110.4391v2

Derrick, G.H. Comments on Nonlinear Wave Equations as Models for Elementary Particles. Journal of Mathematical Physics 5(9):1252 (1964)

D’Inverno, R. Introducing Einstein's Relativity. Claredon Press, Oxford (1992) 
Dodd, R.K., Eilbeck, J.C., Gibbon, J.D., y Morris, H.C. Solitons and Nonlinear Wave Equations. Academic Press Inc., London (1982)

Eilbeck, J.C., Lomdahl, P.S., Olsen, O.H., y Samuelsen, M.R. Comparison Between One-Dimensional and Two-Dimensional Models for Josephson Junctions of Overlap Type. Journal of Applied Physics 57(3):861 (1985)

Erken, O., Sikivie, P., TAm, H., y Yang, Q.L. Axion BEC Dark Matter (2011). arXiv:1111.3976

Erken, O., Sikivie, P., Tam, H., y Yang, Q.L. Cosmic Axion Thermalization. Physical Review D 85(6):063520 (2012). arXiv:1111.1157

FANCHI, J.R. Review of Invariant Time Formulations of Relativistic Quantum Theories. Foundations of Physics 23(3):487-548 (1993)

Felsager, B. Geometry, Particles and Fields. 2ª edición. Odense University Press (1983)

Feynman, R.P. A Relativistic Cut-Off for Classical Electrodynamics. Physical Review 74(8):939-946 (1948a)

Feynman, R.P. Space-Time Approach to Non-Relativistic Quantum Mechanics. Reviews of Modern Physics 20(2):367-387 (1948b)

Feynman, R.P. Space-Time Approach to Quantum Electrodynamics. Physical Review 76(6):769-789 (1949a)

Feynman, R.P. The Theory of Positrons. Physical Review 76(6):749-759 (1949b)

Fordy, A.P. Y GibBons, J. Integrable Nonlinear Klein-Gordon Equations and Toda Lattices. Communications in Mathematical Physics 77(1):21-30 (1980)

Frantzeskakis, D.J. Dark Solitons in Atomic Bose-Einstein Condensates: from Theory to Experiments. Journal of Physics A: Mathematical and Theoretica 213001:82 (2010). arXiv: 1004.4071

Friedberg, R., LeE, T.D., y Sirlin, A. Class of Scalar-Field Soliton Solutions in Three Space Dimensions. Physical Review D 13(10):2739-2761 (1976)

Frieman, J.A., Gelmini, G.B., Gleiser, M., y Kolb, E.W. Primordial Origin of Nontopological Solitons. Physical Review Letters 60(21):2101-2104 (1988)

Fuchs, B. Y Mielke, E.W. Scaling Behaviour of a Scalar Field Model of Dark Matter Haloes. Monthly Notices of the Royal Astronomical Society 350(2):707-709 (2004) 
Greenberg, O. CPT Violation Implies Violation of Lorentz Invariance. Physical Review Letters 89(23):231602 (2002)

Gulevich, D.R., Kusmartsev, F.V., Savel'ev, S., Yampol'skit, V.A., y Nori, F. Shape Waves in 2D Josephson Junctions: Exact Solutions and Time Dilation. Physical Review Letters 101(12):127002 (2008)

Gulevich, D.R., Kusmartsev, F.V., Savel'ev, S., Yampol'skit, V.A., y Nori, F. Shape and Wobbling Wave Excitations in Josephson Junctions: Exact Solutions of the (2+1)-Dimensional sine-Gordon Model. Physical Review B 80(9):094509 (2009)

Hertzberg, M.P., Tegmark, M., y Wilczek, F. Axion Cosmology and the Energy Scale of Inflation. Physical Review D 78(8):083507 (2008)

Hietarinta, J. A Search for Bilinear Equations Passing Hirota's Three-Soliton Condition. I. KdV-type Bilinear Equations. Journal of Mathematical Physics 28(8):1732 (1987a)

Hietarinta, J. A Search for Bilinear Equations Passing Hirota's Three-Soliton Condition. II. mKdV-Type Bilinear Equations. Journal of Mathematical Physics 28(8):2094 (1987b)

Hietarinta, J. A Search for Bilinear Equations Passing Hirota's Three-Soliton Condition. III. sine-Gordon-Type Bilinear Equations. Journal of Mathematical Physics 28(8):2586 (1987c)

Hietarinta, J. A Search for Bilinear Equations Passing Hirota's Three-Soliton Condition. IV. Complex Bilinear Equations. Journal of Mathematical Physics 29(8):628 (1988)

Hirota, R. Exact Solution of the Korteweg-de Vries Equation for Multiple Collisions of Solitons. Physical Review Letters 27(18):1192-1194 (1971). 1011.1669v3

Hirota, R. Exact Solution of the sine-Gordon Equation for Multiple Collisions of Solitons. Journal of the Physical Society of Japan 33(5):1459-1463 (1972)

Hirota, R. The Direct Method in Soliton Theory, Cambridge Tracts in Mathematics, tomo 155. Cambridge University Press, Cambridge (2004)

Infeld, E. Y Rowlands, G. Nonlinear Waves, Solitons and Caos. Cambridge University Press, Cambridge (1990) 
Ivancevic, V.G. Y Ivancevic, T.T. sine-Gordon Solitons, Kinks and Breathers as Physical Models of Nonlinear Excitations in Living Cellular Structures. Journal of Geometry and Symmetry in Physics 31:1-56 (2013). arXiv:1305.0613

KAKu, M. Quantum Field Theory. Oxford University Press, New York (1993)

Khare, A. y Sukhatme, U. Linear Superposition in Nonlinear Equations. Physical Review Letters 88(24):244101 (2002)

Kim, J.E. Weak-Interaction Singlet and Strong CP Invariance. Physical Review Letters 43(2):103-107 (1979)

Kim, J.E. y CARosi, G. Axions and the Strong CP Problem. Reviews of Modern Physics 82(1):557-601 (2010)

Kivshar, Y.S. y Malomed, B.A. Dynamics of Solitons in Nearly Integrable Systems. Reviews of Modern Physics 61(4):763-915 (1989)

Kumar, P. y Thomas, B. Internal Modes of a Soliton. Physical Review B 37(1):683-684 (1988)

Kuster, M., Raffelt, G., y Beltrán, B. (editores). Axions: Theory, Cosmology, and Experimental Searches, Lecture Notes in Physics, tomo 741. Springer, Berlin Heidelberg (2008)

LEE, J.W. Is Dark atter a BEC or scalar field? Journal of the Korean Physical Society 54(6):2622 (2009)

Lee, J.W., Lim, S., y Choi, D. BEC Dark Matter Can Explain Collisions of Galaxy Clusters pág. 4 (2008). arXiv:0805.3827

Lee, J. Y Baldi, M. Can Coupled Dark Energy Speed Up the Bullet Cluster? The Astrophysical Journal 747(1):45 (2011). 1110.0015

Lohe, M.A. Soliton Structures in $P(\phi)_{2}$. Physical Review D 20(12):3120-3130 (1979)

Lomdahl, P.S. Solitons in Josephson Junctions: An Overview. Journal of Statistical Physics 39(5-6):551-561 (1985)

Mahdavi, A., Hoekstra, H., Babul, A., Balam, D.D., y Capak, P. A Dark Core in Abell 520. The Astrophysical Journal (2007). arXiv:0706.3048

Makhankov, V.G. Dynamics of Classical Solitons (In non-integrable systems). Physics Reports 35(1):1-128 (1978) 
Makhankov, V.G. Computer and Solitons. Physica Scripta 20(3-4):558-562 (1979)

Manton, N.S. y Sutcliffe, P.M. Topological Solitons. Cambridge University Press, Cambridge (2004)

Mclaughlin, D.W. y ScotT, A.C. A Restricted Bäcklund Transformation. Journal of Mathematical Physics 14(12):1817 (1973)

Mielke, E.W. Note on Localized Solutions of a Nonlinear Klein-Gordon Equation Related to Riemannian Geometry. Physical Review D 18(12):4525-4528 (1978)

Mielke, E.W. Y Schunck, F.E. Are Axidilaton Stars Massive Compact Halo Objects? General Relativity and Gravitation 33(5):805-813 (2001)

Mielke, E.W. Y Schunck, F.E. Nontopological Scalar Soliton as Dark Matter Halo. Physical Review D 66(2):023503 (2002)

Mielke, E.W. y VÉlez PÉRez, J.A. Toroidal Halos in a Nontopological Soliton Model of Dark Matter. Physical Review D 75(4):043504 (2007)

Mielke, E.W. Y Vélez Pérez, J.A. Axion Condensate as a Model for Dark Matter Halos. Physics Letters B 671(1):174-178 (2009)

Mielke, E.W., Hehl, F.W., y Dermott MCCreA, J. Belinfante Type Invariance of the Noether Identities in a Riemannian and a Weitzenböck Spacetime. Physics Letters A 140(7-8):368-372 (1989)

Mo, H., van Den Bosch, F., Y White, S.D.M. Galaxy Formation and Evolution. Cambridge University Press, New York (2010)

Mullan, D.J. Physics of the Sun. Pure and Applied Physics. CRC Press, Taylor \& Francis Group, Boca Raton (2010)

Munteanu, L. Y Donescu, S. Introduction to Soliton Theory: Applications to Mechanics. Kluwer Academic Publishers, New York (2005)

Myrzakulov, R. Integrable geometry and Soliton equations in $2+1$ dimensions (1998). 9808041

Newell, A.C. Solitons in Matematics and Physics, Regional Conference Series in Applied Mathematics, tomo 48. SIAM, Philadelphia (1985)

Palenzuela, C., Olabarrieta, I., Lehner, L., Y Liebling, S.L. Head-on Collisions of Boson Stars. Physical Review D 75(6):64005 (2007) 
Patani, A., Schlindwein, M., Y Shafi, Q. Topological Charges in fFIeld theory. Journal of Physics A: Mathematical and General 9(9):1513-1520 (1976)

Peccei, R.D. Y Quinn, H.R. CP Conservation in the Presence of Pseudoparticles. Physical Review Letters 38(25):1440-1443 (1977)

Perring, J.K. Y Skyrme, T.H.R. A Model Unified Field Equation. Nuclear Physics 31:550-555 (1962)

Persic, M., Salucci, P., y Stel, F. The Universal Rotation Curve of Spiral Galaxies - I. The Dark Matter Connection. Monthly Notices of the Royal Astronomical Society 281(1):27-47 (1996)

Porter, M.A., Zabusky, N.J., Hu, B., y Campbell, D.K. Fermi, Pasta, Ulam and the Birth of Experimental Mathematics. American Scientist 97:214 (2009)

Preskill, J., Wise, M.B., Y Wilczek, F. Cosmology of the Invisible Axion (1983)

Rajaraman, R. Solitons and Instantons. North-Holland, Amsterdam (1982)

Remoissenet, M. Waves Called Solitons. 2a edición. Springer-Verlag, Berlin Heidelberg (1996)

Riotto, A. Y Tkachev, I. What if Dark Matter is Bosonic and Self-Interacting. Physics Letters B 484(3-4):177-182 (2000)

Rubinstein, J. sine-Gordon Equation. Journal of Mathematical Physics 11(1):258 (1970)

SARkAR, U. Particle and Astroparticle Physics. Series in High Energy Physics, Cosmology and Gravitation. Taylor \& Francis Group, New York, London (2008)

Schunck, F.E. A Scalar Field Matter Model for Dark Halos of Galaxies and Gravitational Redshift (1998). 9802258

Schunck, F.E. y Mielke, E.W. General Relativistic Boson Stars. Classical and Quantum Gravity 20(20):R301-R356 (2003)

Scott, A.C., Chu, F.Y.F., Y Mclaughlin, D.W. The Soliton: A New Concept in Applied Science. Proceedings of the IEEE 61(10):1443-1483 (1973)

Sikivie, P. An Argument that the Dark Matter is Axions. arXiv (2012). 1210.0040

Sikivie, P. Y YAng, Q.L. Bose-Einstein Condensation of Dark Matter Axions. Physical Review Letters 103(11):111301 (2009) 
Sin, S.J. Late-Time Phase Transition and the Galactic Halo as a Bose Liquid. Physical Review D 50(6):3650-3654 (1994)

Sozzi, M.S. Discrete Symmetries and CP Violation: From Experiment to Theory. Oxford Graduate Texts. Oxford University Press (2008)

Spergel, D. Y Steinhardt, P.J. Observational Evidence for Self-Interacting Cold Dark Matter. Physical Review Letters 84(17):3760-3763 (2000)

Stephani, H. Differential Equation: their Solution Using Symmetries. Cambridge University Press (1989)

Stueckelberg, E.C.G. Remarks on the Creation of Pairs of Particles in the Theory of Relativity. Helvetica Physica Acta 14:588-594 (1941)

Stueckelberg, E.C.G. The Mechanics of Point Particles in the Theory of Relativity and the Quantum Theory. Helvetica Physica Acta 15:23 (1942)

Taniuti, T. Y Nishinara, K. Nonlinear Waves, Monographs and Studies in Mathematics, tomo 15. Pitman Advanced Publishing Program, London (1983)

TER HAAR, D. Solitons. Physica Scripta 20(3-4):291-295 (1979)

Ustinov, A.V. Solitons in Josephson Junctions. Physica D 123(1-4):315-329 (1998)

Vogel, J.K., Armengaud, E., Avignone, F.T., Betz, M., Brax, P., Brun, P., Cantatore, G., Carmona, J.M., Carosi, G.P., Caspers, F., Caspi, S., Cetin, S.A., Chelouche, D., Christensen, F.E., Dael, A., Dafni, T., Davenport, M., Derbin, A.V., Desch, K., Diago, A., Döbrich, B., Dratchnev, I., Dudarev, A., Eleftheriadis, C., Fanourakis, G., Ferrer-Ribas, E., Galán, J., GarCía, J.A., Garza, J.G., Geralis, T., Gimeno, B., Giomataris, I., Gninenko, S., Gómez, H., González-Díaz, D., Guendelman, E., Hailey, C.J., Hiramatsu, T., Hoffmann, D.H.H., Horns, D., Iguaz, F.J., Irastorza, I.G., Isern, J., Imai, K., Jakobsen, A.C., Jaeckel, J., JakovČıć, K., Kaminski, J., Kawasaki, M., Karuza, M., KrČmar, M., Kousouris, K., Krieger, C., Lakić, B., Limousin, O., Lindner, A., Liolios, A., Luzón, G., Matsuki, S., Muratova, V.N., Nones, C., Ortega, I., Papaevangelou, T., Pivovaroff, M.J., Raffelt, G., Redondo, J., Ringwald, A., Russenschuck, S., Ruz, J., Saikawa, K., SavviDis, I., Sekiguchi, T., Semertzidis, Y.K., Shilon, I., Sikivie, P., Silva, H., Ten Kate, H., Tomas, A., Troitsky, S., Vafeiadis, T., Van Bibber, K., Vedrine, P., Villar, J.A., Walckiers, L., Weltman, A., Wester, W., Yildiz, S.C., Y 
Zioutas, K. The Next Generation of Axion Helioscopes: The International Axion Observatory (IAXO). Physics Procedia 61(0):193-200 (2015)

Weinberg, S. The U(1) Problem. Physical Review D 11(12):3583-3593 (1975)

Weinberg, S. A New Light Boson? Physical Review Letters 40(4):223-226 (1978)

WeIssert, T.P. The Genesis of Simulation in Dynamics. Springer New York (1997)

Whitham, G.B. Linear and Nonlinear Waves. Pure and applied mathematics. WileyInterscience, New York (1974)

WilczeK, F. Problem of Strong $\mathrm{P}$ and $\mathrm{T}$ Invariance in the Presence of Instantons. Physical Review Letters 40(5):279-282 (1978)

Zioutas, K., Hoffmann, D.H.H., Dennerl, K., Y Papaevangelou, T. What is Dark Matter Made of? Science 306(5701):1485-8 (2004)

Zwicky, F. Die Rotverschiebung von Extragalaktischen Nebeln. Helvetica Physica Acta 6:110-127 (1933)

ZwICKY, F. Republication of: The Redshift of Extragalactic Nebulae. General Relativity and Gravitation 41(1):207-224 (2008) 



\section{Apéndice A}

\section{Teoría de campos}

La introducción de campos (elementos extendidos espacio-temporalmente) en lugar de partículas puntuales (puntos materiales), se ve principalmente reflejada en la cantidad de grados de libertad, ya que mientras para partículas localizadas $\vec{x}_{A}$ los grados de libertad para un sistema son proporcionales al número de partículas, mientras que para los campos, cada campo $\phi^{A}(\vec{x})$ tiene infinitos grados de libertad $\left(\infty^{d}\right)$.

\section{A.1. Campo escalar}

La dinámica clásica de campos está determinada por la acción, por tal razón las condiciones se establecen sobre ésta. Las principales condiciones son

1. Invariancia relativista. En el espacio plano de Minkowski la acción debe ser un invariante de Poincaré, es decir, no debe cambiar de forma ante las transformaciones de Lorentz y las traslaciones. Además, el volumen es invariante ante transformaciones de Lorentz propias y traslaciones, y la densidad lagrangiana $\mathcal{L}$ debe depender de las correspondientes invariantes.

2. Localidad. Los campos dependen de un mismo punto $x^{\mu}$, lo que implica que no hay acción a distancia.

3. Carácter real o hermiciano. La acción involucra sólo combinaciones reales de los campos y sus derivadas. En caso contrario, la acción adquiere una parte imaginaria, y junto a esto la energía seria compleja, lo cual, desde el punto de vista de la teoría cuántica, indicaría la creación y absorción de partículas.

4. Las ecuaciones de campo son a lo más de segundo orden en las derivadas.

El Lagrangiano más simple para el campo escalar es el llamado Klein-Gordon lineal

$$
\mathcal{L}_{\mathrm{KG}}=\frac{1}{2} \partial^{\mu} \phi \partial_{\mu} \phi-\frac{1}{2} m^{2} \phi^{2}
$$


que posee una solución en forma de onda monocromática

$$
\phi(x)=A \mathrm{e}^{-i p x},
$$

un caso más general, es considerar una Lagrangiana de la forma

$$
\mathcal{L}=\frac{1}{2} \partial^{\mu} \phi \partial_{\mu} \phi-V(\phi)
$$

Ésta tiene una separación natural en dos términos, uno cinético $K=\frac{1}{2} \partial_{\mu} \phi \partial^{\mu} \phi$, y el potencial $V(\phi)$. Este potencial es en general una función semi-positiva definida de $\phi$, en la cual, términos cuadráticos corresponden a efectos tipo Klein-Gordon y términos extras corresponden a autointeracción de los campos, en ésta los puntos críticos son llamados soluciones de vacío.

En teoría de partículas, el campo escalar o pseudoescalar representa partículas con espín cero. Partículas como el bosón de Higgs y los axiones. El caso más simple, en teoría relativista es el campo escalar real, esto es, un campo de una sola componente $\psi=\psi(x)$, invariante respecto a las transformaciones de Poincaré.

\section{A.2. Formulación Lagrangiana}

La interacción en sistemas físicos se puede exponer en términos de la función Lagrangiana del sistema, en el caso de teoría de campo esta función escalar es generalizada a través de una densidad Lagrangiana $\mathcal{L}$, que es función del campo de cada partícula $\phi(x)$ o posibles componentes $\phi^{A}(x)$ del campo y las derivadas espacio-temporales $\partial_{\mu}:=\partial / \partial x^{\mu}$ de los mismos, para un sólo campo tenemos

$$
\mathcal{L}=\mathcal{L}\left(\phi, \partial_{\mu} \phi\right)
$$

Integrar $\mathcal{L}$ sobre todo el volumen espacial donde existe el campo, nos lleva a la Lagrangiana, e integrando ésta en un intervalo temporal a la acción

$$
\begin{aligned}
L & =\int d^{3} x \mathcal{L}\left(\phi, \partial_{\mu} \phi\right), \\
S & =\int d^{4} x \mathcal{L}=\int d t L .
\end{aligned}
$$

El principio de mínima acción implica calcular la variación

$$
\delta S[\phi]=\frac{d}{d \epsilon}(S[\phi+\epsilon \delta \phi])_{\epsilon=0}=\int d^{4} x \delta \mathcal{L}\left(\phi, \partial_{\mu} \phi\right)=0,
$$

que nos conduce a las ecuaciones de movimiento

$$
\delta \mathcal{L}\left(\phi, \partial_{\mu} \phi\right)=\frac{\delta \mathcal{L}}{\delta \phi} \delta \phi+\frac{\delta \mathcal{L}}{\delta \partial_{\mu} \phi} \delta \partial_{\mu} \phi
$$


que integrando por partes se transforma y se pueden distinguir dos contribuciones

$$
\delta \mathcal{L}\left(\phi, \partial_{\mu} \phi\right)=\left(\frac{\delta \mathcal{L}}{\delta \phi}-\partial_{\mu} \frac{\delta \mathcal{L}}{\delta \partial_{\mu} \phi}\right) \delta \phi+\partial_{\mu}\left(\frac{\delta \mathcal{L}}{\delta \partial_{\mu} \phi} \delta \phi\right) .
$$

Introduciendo esto en (A.2.4), el primer término nos lleva a las ecuaciones de EulerLagrange

$$
\frac{\delta \mathcal{L}}{\delta \phi}=\partial_{\mu} \frac{\delta \mathcal{L}}{\delta \partial_{\mu} \phi} .
$$

mientras que la segunda contribución es un término de frontera que se anula, lo que se muestra utilizando el teorema de Gauss

$$
\int d^{4} x\left[\partial_{\mu}\left(\frac{\delta \mathcal{L}}{\delta \partial_{\mu} \phi} \delta \phi\right)\right]=\int_{\partial} d S_{\mu}\left(\frac{\delta \mathcal{L}}{\delta \partial_{\mu} \phi} \delta \phi\right),
$$

ya que $\delta \phi$ debe anularse en la frontera de integración. La generalización a múltiples componentes del campo, se logra sustituyendo el campo $\phi$ por cada campo $\phi^{A}$, que debe satisfacer la ecuación (A.2.7).

Aplicando la ecuación de Euler-Lagrange (A.2.7) a la densidad Lagrangiana (A.1.3) obtenemos la ecuación de movimiento

$$
\square \phi=\partial_{\mu} \partial^{\mu} \phi=-\frac{\partial V(\phi)}{\partial \phi}
$$

donde $\square:=\partial_{\mu} \partial^{\mu}=c^{-2} \partial_{t t}-\nabla^{2}$, es el operador D'Alembertiano, en ocasiones llamado operador de onda.

La ecuación de movimiento resultante (A.2.9), es en este caso una ecuación de onda, y depende de la elección del potencial $V(\phi)$, con el cual podemos obtener una ecuación de onda libre, lineal o no lineal. El ejemplo más simple (campo relativista real), nos lleva a la ecuación de Klein-Gordon, que se obtiene cuando el potencial tiene la forma $V(\phi)=m^{2} \phi^{2} / 2$, así tenemos

$$
\square \phi+m^{2} \phi=0
$$

que describe el movimiento de una partícula puntual relativista.

Haciendo una translación en el campo $\phi(x) \rightarrow \phi(x+a)$, al variación la podemos expresar utilizando un desarrollo en serie de potencias para $x^{\mu}$ como sigue

$$
\delta \phi \rightarrow \phi(x+a)-\phi(x) \approx a^{\mu} \partial_{\mu} \phi(x)
$$

donde $\delta x^{\mu}=a^{\mu}$ y de manera análoga

$$
\delta \partial_{\mu} \phi \rightarrow \partial_{\mu} \phi(x+a)-\partial_{\mu} \phi(x) \approx a^{\nu} \partial_{\nu} \partial_{\mu} \phi(x),
$$

con esto el cambio en la Lagrangiana es

$$
\mathcal{L} \rightarrow \mathcal{L}+\left(\frac{\delta \mathcal{L}}{\delta \phi} \delta \phi+\frac{\delta \mathcal{L}}{\delta \partial_{\mu} \phi} \delta \partial_{\mu} \phi\right)=\mathcal{L}+a^{\nu} \partial_{\mu}\left(\frac{\delta \mathcal{L}}{\delta \partial_{\mu} \phi} \partial_{\nu} \phi\right)
$$


que también puede expresarse con la variación de la Lagrangiana

$$
\delta \mathcal{L} \rightarrow \mathcal{L}\left(\phi+\delta \phi, \partial_{\mu} \phi+\delta \partial_{\mu} \phi\right)-\mathcal{L}\left(\phi, \partial_{\mu} \phi\right) \approx a^{\nu} \partial_{\nu} \mathcal{L}\left(\phi, \partial_{\mu} \phi\right)
$$

entonces el

$$
\mathcal{L} \rightarrow \mathcal{L}+a^{\nu} \partial_{\nu} \mathcal{L}=\mathcal{L}+a^{\nu} \partial_{\mu}\left(\delta_{\nu}^{\mu} \mathcal{L}\right)
$$

esta ecuación nos dice que hay una corriente por cada $a^{\nu}$, entonces combinando (A.2.14) y (A.2.15) obtenemos

$$
a^{\nu} \partial_{\mu}\left(\frac{\delta \mathcal{L}}{\delta \partial_{\mu} \phi} \partial_{\nu} \phi-\mathcal{L} \delta_{\nu}^{\mu}\right)=0
$$

que nos define una cantidad conservada llamada tensor de energía-momento canónico

$$
\Theta^{\mu}{ }_{\nu}:=\mathcal{L} \delta_{\nu}^{\mu}-\frac{\delta \mathcal{L}}{\delta \partial_{\mu} \phi} \partial_{\nu} \phi .
$$

que no es necesariamente simétrico.

Debido a que el tensor de energía-momento no es una cantidad directamente medible, mientras que sus integrales, las cargas, si lo son; se genera una ambigüedad en la definición del tensor de energía-momento, ya que mientras la conservación de momento angular requiere que el tensor $\Theta^{\mu}{ }_{\nu}$ sea simétrico, la conservación de momento no lo requiere. Para resolver esta ambigüedad se puede añadir un término extra de la forma $\partial_{\lambda} E^{\lambda \mu \nu}$, lo que genera un nuevo tensor simétrico de energía-momento llamado tensor de energía-momento de Belinfante-Rosenfeld

$$
\Theta^{\mu \nu} \rightarrow \Theta^{\mu \nu}+\partial_{\lambda} E^{\lambda \mu \nu}
$$

donde $E^{\lambda \mu \nu}=-E^{\mu \lambda \nu}$ es un superpotencial y su antisimetría nos lleva a que

$$
\partial_{\lambda} \partial_{\mu} E^{\lambda \mu \nu}=0
$$

con esto la ley de conservación momento se reduce a

$$
\partial_{\mu} T^{\mu \nu}=\partial_{\mu}\left(\Theta^{\mu \nu}+\partial_{\lambda} E^{\lambda \mu \nu}\right)=\partial_{\mu} \Theta^{\mu \nu}=0
$$

De esta manera el tensor de energía-momento es de la forma

$$
T_{\mu \nu}=\partial_{\mu} \phi \partial_{\nu} \phi-\eta_{\mu \nu} \mathcal{L}(\phi)
$$

cuando $\eta_{\mu \nu}$ es la métrica $(+1,-1)$, los componentes son

$$
\begin{aligned}
T_{00} & =\frac{1}{2}\left(\phi_{t}^{2}+\phi_{x}^{2}\right)+V(\phi) \\
T_{11} & =\frac{1}{2}\left(\phi_{t}^{2}+\phi_{x}^{2}\right)-V(\phi) \\
T_{10}=T_{01} & =\phi_{x t}
\end{aligned}
$$


y los componentes contravariantes tienen la forma $T^{\mu \nu}$

$$
\begin{aligned}
& T^{00}=T_{00} \\
& T^{11}=T_{11} \\
& T^{10}=-T_{01}
\end{aligned}
$$

que son las cargas de Noether respecto a la simetría de translación espacial y simetría de translación temporal, respectivamente. Otra razón para que el tensor de energía-momento sea simétrico, se debe a que en relatividad general, el tensor de campo gravitacional es simétrico, debido al principio de equivalencia, además que la ecuación de Einstein es también simétrica.

En la construcción de las teorías de campo, se a visto que el estudio de las simetrías juega una gran importancia. De hecho, el estudio de las simetrías ha mostrado ser la herramienta más poderosa para describir el universo físico.

La energía del campo que obedece la densidad Lagrangiana (A.1.3), la obtenemos a partir de la integral

$$
E[\phi]=\int_{-\infty}^{\infty} \rho\left(t, x_{i}\right) d x_{i},
$$

donde $\rho(t, x)$ es la densidad de energía

$$
\rho\left(c t, x_{i}\right)=T^{00}=\frac{1}{2}\left(\frac{\partial \phi}{\partial x^{0}}\right)^{2}+\frac{1}{2}\left(\frac{\partial \phi}{\partial x^{i}}\right)^{2}+V(\phi)
$$

en esencia, tenemos la suma de las densidades de energía cinética y potencial.

Notemos que las ecuaciones (A.1.3), (A.2.23) y (A.2.9) tienen las simetrías $P$ y $T$, mientras que simetrías respecto al campo dependen de la elección del potencial. Además, si la dinámica es no disipativa podemos utilizar el formalismo Lagrangiano; vid. Kaku (1993).

Partiendo del tensor de energía-momento se puede definir el tensor de momento orbital

$$
\mathcal{M}^{\rho \mu \nu}:=\Theta^{\rho \nu} x^{\mu}-\Theta^{\rho \mu} x^{\nu}
$$

\section{A.3. Simetrías en la naturaleza}

Los sistemas en la naturaleza presentan simetrías como un medio para la manifestación de algunas características físicas. Estas simetrías pueden ser locales o globales; esto es, se mantienen para algunos o todos los puntos del espacio-tiempo. Así también, pueden ser continuas y discretas; es decir, depender de la cantidad de transformaciones que pueden aplicarse al sistema y dejarlo invariante. 
Las simetrías continuas de espacio-tiempo se manifiestan como principios de conservación, donde interviene una corriente conservada que puede manifestarse como una o más cargas conservadas; estas se estudian con el teorema de Noether. Mientras que simetrías locales se pueden estudiar con teoría de norma, éstas están descritas por $n$ generadores. Teoría en la qué, cuando los generadores conmutan entre si se dice Abeliana, y en caso contrario se dice no-Abeliana. Notemos que, todas las interacciones fundamentales se presentan en simetrías locales continuas.

Simetrías discretas se presenta cuando la cantidad de transformaciones que dejan invariante el sistema es finita o numerable, éstas pueden no conducir a corrientes conservadas, por lo que no conciernen al teorema de Noether.

Una descripción más matemática de las simetrías es utilizando la teoría de grupo, mientras que las simetrías continuas son descritas por grupos de Lie y las simetrías discretas por grupos finitos. Muchas simetría, además de dejar la acción invariante, dejan también el Lagrangiano invariante; por ejemplo, translaciones y rotaciones espaciales, transformaciones de isospín, color, sabor; así como otras simetrías internas independientes de la simetrías del espacio-tiempo.

Otra clasificación entre simetrías útil, es en simetrías internas, espacio-temporales y de isospín. Las simetrías internas, mezclan las componentes del campo entre ellas. Rotan campos y partículas en un espacio isotrópico abstracto $S O(n)$, donde $n$ es el número de partículas, en contraste al real espacio-tiempo. Son compactas y el rango de sus parámetros es finito y contienen sus puntos finales. Por su parte, las simetrías espacio-temporales, son aquellas del tipo de los grupos de Lorentz y Poincaré. Son no compactas, es decir, los parámetros no contienen sus puntos finales. El tercer tipo las simetrías de isospín, están relacionadas con la interacción fuerte y de partículas como bariones y mesones. La simetría del isospín es un subconjunto de simetría del sabor visto más ampliamente en las interacciones de bariones y mesones.

\section{A.3.1. Simetrías Continuas y teorema de Noether}

Las simetrías continuas de espacio-tiempo se manifiestan como principios de conservación, donde interviene lo que llamamos una corriente conservada que puede manifestarse como una o más cantidades conservadas; estas se estudian con el teorema de Noether, y tienen una estructura definida en la teoría de grupos de Lie. Los generadores del grupo representan los observables conservados y sus relaciones de conmutación son inequívocamente determinadas por la estructura de éste. 


\section{A.3.1.1. Teorema de Noether}

El teorema planteado por Emmy Noether (1918) nos permite identificar principios de conservación mediante simetrías continuas de la acción. Por ejemplo, si en el Hamiltoniano tenemos que la independencia respecto al tiempo, la que indica la conservación de la energía. Y similarmente, la invariancia ante translaciones y rotaciones nos conducen a la conservación del momento lineal y momento angular, respectivamente. Sin embargo, no dice nada respecto a simetrías discretas, por lo que éstas no necesariamente llevan a leyes de conservación, es decir a cantidades conservadas; vid. Sozzi (2008).

Considerando las ecuaciones de Euler-Lagrange (A.2.7) en la variación de de la densidad Lagrangiana (A.2.6), obtenemos la ecuación de Noether

$$
\delta \mathcal{L}\left(\phi, \partial_{\mu} \phi\right)=\partial_{\mu}\left(\frac{\delta \mathcal{L}}{\delta \partial_{\mu} \phi} \delta \phi\right) .
$$

Ecuación que nos permite definir la corriente de Noether como

$$
J^{\mu}:=\frac{\delta \mathcal{L}}{\delta \partial_{\mu} \phi} \delta \phi
$$

ésta a su vez, asocia una ley de conservación puede escribirse como

$$
\partial_{\mu} J^{\mu}=\partial_{0} J^{0}-\partial_{i} J^{i}=0
$$

De ésta, integrando podemos identificar una cantidad conservada que se conoce como carga de Noether

$$
Q:=\int_{-\infty}^{\infty} d^{3} x J^{0}
$$

que es independiente del tiempo, ya que

$$
\frac{d Q}{d t}=\int_{-\infty}^{\infty} d^{3} x \partial_{0} J^{0}=\int_{-\infty}^{\infty} d^{3} x \partial_{i} J^{i}=\left.J^{\prime}\right|_{\text {frontera }}=0
$$

En el caso que la Lagrangiana dependa de varios campos $\phi^{A}$, realizando las variaciones sobre cada campo, se pueden obtener leyes de conservación análogas para cada uno de los campos.

La ley de conservación más importantes debidas a este teorema es con el tensor de momento orbital

$$
\partial_{\rho} \mathcal{M}^{\rho \mu \nu}=0
$$

y la carga

$$
M^{\mu \nu}=\int d^{3} x \mathcal{M}^{0 \mu \nu}
$$

Estas cargas forman un conjunto conocido como generadores del grupo homogéneo de Lorentz. Por la antisimetría $\mu \neq \nu$, basta con los casos: $(i) \mu=i$ y $\nu=0,(i i) \mu=0$ y 
$\nu=i \mathrm{y}(i i i) \mu=i \mathrm{y} \nu=j$. Los caso $(i)$ y $(i i)$ son antisimétricos uno al otro, así tomamos $\mu=i \mathrm{y} \nu=0$,

$$
M^{i 0}=\int d^{3} x \mathcal{M}^{0 i 0}=\int d^{3} x\left(\Theta^{00} x^{i}-\Theta^{0 i} x^{0}\right)=-M^{0 i}
$$

estos casos dependen explícitamente de la coordenada temporal. Lo que permite definir los llamados generadores boost

$$
K_{i}:=\int d^{3} x\left(\Theta^{00} x^{i}\right)-\mathcal{P}^{i} x^{0}
$$

donde

$$
\mathcal{P}^{\mu}:=\int d^{3} x \Theta_{0}^{\mu}
$$

en el caso $(i i i)$ con $\mu=i$ y $\nu=j$

$$
M^{i j}=\int d^{3} x \mathcal{M}^{0 i j}=\int d^{3} x\left(\Theta^{0 j} x^{i}-\Theta^{0 i} x^{j}\right),
$$

permite definir los llamados generadores de rotación

$$
J_{k}:=\frac{1}{2} \epsilon_{i j k} M^{i j}
$$

Las relaciones de conmutación entre $K_{k}, J_{k}$ y $P^{\mu}$ definen el álgebra de Poincaré.

\section{A.3.2. Simetrías Discretas y Invarianza $C P T$}

Las simetrías discretas más comunes se presentan en términos como $y^{2}, y^{3}, y^{4}$, etc., éstas corresponden a los grupos $Z_{2}, Z_{3}, Z_{4}$, etc... En particular en el grupo $Z_{2}$, las simetrías generadas son de la forma $y \rightarrow-y$, las más importantes que pertenecen a este grupo son la paridad $P$, la inversión temporal $T$ y la conjugación de carga $C$. Estas tres, son de gran importancia en la física debido al llamado teorema $C P T$. Esté establece que el producto de las tres transformaciones es una simetría valida para cualquier sistema físico, i.e. es una invarianza de la naturaleza. Dicha invarianza se presenta sin importar el orden de aplicación de cada transformación y es la única combinación que a la fecha es observada como una simetría exacta de la naturaleza.

El teorema $C P T$ provee la justificación a la interpretación de Stueckelberg-Feynman de soluciones con energía negativa, las cuales viajan hacia atrás en el espacio-tiempo, como antipartículas pero con energía negativa $E>0$ y carga opuesta $C$. La simetría $C P T$ requiere que si una de las simetrías es violada, al menos una más también lo es. Por esto, la violación de $C P$ se dice ser equivalente a una violación de $T$.

Algunas de las principales consecuencias del la simetría $C P T$ pueden resumirse en que: 
I) las partículas y antipartículas deben tener masas iguales,

II) las partículas y antipartículas tienen el mismo tiempo de vida,

III) las antipartículas deben existir, inclusive si la conjugación de la carga no es una simetría exacta de la naturaleza.

Por ejemplo, para un campo escalar la simetría CPT transforma tal que

$$
\phi(t, \vec{x}) \rightarrow \phi_{C P T}(t, \vec{x})=\eta_{C P T} \phi(-t,-\vec{x})
$$

donde $\eta_{C P T}=+1$, y $\eta_{C P T}=-1$ en el caso pseudoescalar. Tanto la Lagrangiana $\mathrm{y}$ Hamiltoniana de un sistema se transforman bajo CPT como

$$
\begin{aligned}
& (C P T) \mathcal{L}(x)(C P T)^{-1}=\mathcal{L}(-x) \\
& (C P T) \mathcal{H}(x)(C P T)^{-1}=\mathcal{H}(-x)
\end{aligned}
$$

Greenberg (2002) ha mostrado que una hipotética violación de la simetría CPT, llevaría al rompimiento de la simetría de Lorentz, que si bien, no son equivalentes ambas son las más importantes en la naturaleza; además de ser dos piedras fundamentales de la teoría cuántica de campos.

\section{A.3.2.1. Transformación de paridad}

El efecto de la transformación de paridad es definido como la inversión de las coordenadas espaciales con respecto al origen

$$
P: \vec{x} \rightarrow \vec{x}_{P}=-\vec{x}
$$

que en su representación pasiva corresponde a la reversión de los tres ejes espaciales, bajo la cual un sistema derecho se transforma en un sistema izquierdo (y vice versa), y es llamada inversión espacial. Esta simetría está asociada a la isotropía del espacio, por lo que se espera que siempre se cumpla.

En los vectores de momento y momento angular la paridad se refleja como

$$
\vec{p} \rightarrow \vec{p}_{P}=-\vec{p}, \quad \vec{L} \rightarrow \vec{L}_{P}=+\vec{L}
$$

aquí hay que notar el hecho de que el vector $\vec{p}$ de momento es un vector polar, mientras que el momento angular $\vec{L}$ es un vector axial. Esta clasificación se da precisamente por la aplicación del operador de paridad; son vectores polares los que son invertidos por la transformación de paridad y son vectores axiales los que no.

Esto mismo puede aplicarse a los campos eléctrico y magnético, tal que

$$
\vec{E}(t, \vec{x}) \rightarrow-\vec{E}(t,-\vec{x}), \quad \vec{B}(t, \vec{x}) \rightarrow+\vec{B}(t,-\vec{x})
$$


esto se debe cumplir para lograr la invarianza de las ecuaciones de Maxwell.

Mientras que la paridad es una transformación que conmuta con las rotaciones, y es cualitativamente diferente a aquellas, una transformación de paridad no puede ser obtenida por la combinación de rotaciones, debido a que el número de coordenadas es impar. Matemáticamente, el determinante de las matrices de paridad $M$ es -1 , mientras que para las rotaciones es +1 .

Para un campo escalar

$$
\phi(t, \vec{x}) \rightarrow \phi_{P}(t, \vec{x})=\eta_{P} \phi(t,-\vec{x})
$$

donde $\eta_{P}= \pm 1$ es una factor de fase, en ocasiones llamado paridad intrínseca.

No hay evidencia de violación de paridad en electromagnetismo ni en interacciones fuertes, sin embargo en interacciones débiles si la hay; y significa que las amplitudes de los estados ligados de paridad opuesta son tan grandes como aquellos ligados en la misma paridad.

\section{A.3.2.2. Inversión temporal}

La transformación corresponde a la sustitución formal de

$$
T: t \rightarrow t_{T}=-t
$$

si bien, en nuestro mundo macroscópico el tiempo es regido por la llamada flecha del tiempo, que nos da una dirección aparente en todos los procesos físicos, y es de hecho irreversible. En la física clásica sin embargo, por ejemplo en las leyes de Newton la inversión del tiempo parece ser un invariante, esto ya que tratamos con la interacción de muchas interacciones elementales. Esto parece ser una paradoja: los sistemas macroscópicos en la termodinámica no comparten la reversión del tiempo de los sistemas microscópicos que lo componen.

Esta paradoja se resuelve al comprender que clásicamente cualquier movimiento permitido por las ecuaciones dinámicas es posible, mientras que macroscópicamente tenemos consideraciones termodinámicas que permiten o no la reversibilidad, es decir la simetría de las leyes microscópicas de la física, no tienen nada que ver con la reversibilidad de los procesos.

Bajo esta transformación las coordenadas, el momento y el momento angular se transforma como sigue

$$
\vec{x} \rightarrow \vec{x}_{T}=\vec{x}, \quad \vec{p} \rightarrow \vec{p}_{T}=-\vec{p}, \quad \vec{L} \rightarrow \vec{L}_{T}=-\vec{L}
$$

esto en mecánica clásica. Por otra parte, en electromagnetismo

$$
\vec{E}(t, \vec{x}) \rightarrow+\vec{E}(-t, \vec{x}), \quad \vec{B}(t, \vec{x}) \rightarrow-\vec{B}(-t, \vec{x})
$$


estas transformaciones pueden entenderse del hecho que el campo eléctrico puede crearse por cargas estacionarias (sin importar la reversión del tiempo), mientras el campo magnético es creado por cargas en movimiento.

Para un campo escalar

$$
\phi(t, \vec{x}) \rightarrow \phi_{T}(t, \vec{x})=\eta_{T} \phi^{\dagger}(-t, \vec{x})
$$

donde $\eta_{T}$ es una factor de fase.

La primera (y al parecer la única) medición directa de violación de reversión temporal en interacciones débiles fue obtenida por el estudio de transiciones virtuales de kaones neutrales.

\section{A.3.2.3. Conjugación de carga}

Esta simetría emerge naturalmente del concepto de antipartícula y puede entenderse como, que bajo esta transformación, una partícula se convierte en antipartícula. Se relaciona más con las inversiones de espacio-tiempo, que con el espacio-tiempo como tal, e implica que sólo estados neutros pueden ser invariantes.

Bajo esta transformación todas las cargas (como eléctrica y topológica) cambian de signo, mientras que otras cantidades como las relacionadas con el espacio-tiempo permanecen sin cambio. Una diferencia importante es que la carga no es una variable dinámica, es más bien un parámetro (geométrico) que mecánicamente no es necesario introducir en las ecuaciones de movimiento.

Para campos escalares cargados $\phi$, la ecuación de conjugación de carga del campo es

$$
\phi(t, \vec{x}) \rightarrow \phi_{C}(t, \vec{x})=\eta_{C} \phi^{\dagger}(t, \vec{x})
$$

donde $\eta_{C}$ es un factor de fase, que se refleja en el hecho de que puede haber muchos Hamiltonianos equivalentes describiendo el mismo sistema físico, cada uno para distinto factor de fase. La fase intrínseca no tiene significado físico y no puede ser medida en términos absolutos o relativos a otro campo.

Las principales violaciones se dan en interacciones débiles y están ligadas a violaciones de paridad, por esta razón es común que se estudie la combinación de simetrías $C P$.

\section{A.3.2.4. Reflexión fuerte}

Intuitivamente, ya que el espacio-tiempo posee un número par de dimensiones se pude pensar que $J=P T$ es equivalente a una rotación en $4 \mathrm{D}$, bajo la cual un 4-vector es revertido, y puede ser obtenida como una transformación de Lorentz, una simetría valida. 
Sin embargo, esto no sucede debido a que la naturaleza pseudoeuclidiana del espaciotiempo, en la cual el espacio y el tiempo se comportan diferente: mientras el momento se revierte y la energía negativa esta asociada a las antipartículas.

El efecto de una transformación $J$, también llamada reflexión fuerte, es la inversión de todas sus coordenadas $x^{\mu} \rightarrow-x^{\mu}$, y sus derivadas $\partial_{\mu} \rightarrow-\partial_{\mu}$.

\section{A.4. Interpretación de Stueckelberg-Feynman}

El concepto de antipartícula se origina de la mecánica relativista, ya que el elemento de línea definido del invariante de Lorentz $d s^{2}:=d x_{\mu} d x^{\mu}$ a través de la raíz cuadrada, es tal que para una partícula en reposo admite ambos signos $d \tau=d s / c= \pm d t / \gamma$. En el caso con signo menos, una partícula tiene tiempo propio y coordenada temporal en direcciones opuestas, ésta es interpretada como una antipartícula viajando hacia atrás en el tiempo. Es decir, una partícula que originalmente tiene energía negativa $E<0$, corresponde a una partícula con $E>0$, pero con carga opuesta y sujeto a la la reflexión fuerte $J$ al propagarse.

Primeramente Ernst Stueckelberg (1941, 1942), estudiando la ecuación clásica relativista para una partícula que se mueve en un campo electromagnético. Notó, que ésta era invariante ante el cambio de signo de la carga y de las direcciones espacio-temporales, la interpretación de esto lo llevó a pensar que una partícula cargada (v.g. electrón) y su antipartícula (v.g. positrón) se propagaban en direcciones opuestas tanto espaciales como temporales. Posteriormente Richard P. Feynman (1948b), presento argumentos similares para una acción clásica; vid. Fanchi (1993) 


\section{Apéndice $B$}

\section{Transformaciones y grupos de Lorentz}

En relatividad especial, las coordenadas $x^{\mu}=\left(x^{0}, x^{1}, x^{2}, x^{3}\right)$ permiten localizar un evento en el espacio-tiempo, estas son medidas en distintos sistemas inerciales de referencia y están relacionadas por una transformación de Lorentz. Estas transformaciones son las que nos permitirán evaluar la invariancia relativista.

\section{B.1. Transformaciones de Lorentz}

Las transformaciones se obtienen del hecho de que la distancia $r$ y el tiempo de propagación de una onda esférica satisfacen la relación

$$
\vec{r}^{2}=c t^{2}
$$

dicha medición debe ser invariante en cualquier sistema de coordenadas,

$$
\vec{r}^{2}=c^{2} t^{\prime 2}
$$

donde $c$ es la velocidad de la luz que es la misma en cualquier ambos sistemas de referencia. La combinación de estas expresiones permite definir un intervalo espacio-temporal

$$
s^{2}=c^{2} t^{2}-\vec{r}^{2}=c^{2} t^{2}-\vec{r}^{2}=s^{\prime 2},
$$

dicha relación, debe satisfacerse tanto para translaciones como para rotaciones, tanto espaciales como temporales. Como caso particular consideraremos un pseudogiro o giro hiperbólico, en el plano $(c t, x)$ esta rotación se puede escribir como

$$
\begin{aligned}
x^{\prime} & =x \cosh \theta-c t \sinh \theta \\
c t^{\prime} & =-x \sinh \theta+c t \cosh \theta,
\end{aligned}
$$


la relación entre los parámetros $\theta$ y $v$ la velocidad a la que se mueve $x^{\prime}=0$, son

$$
\begin{aligned}
\tanh \theta & =\frac{v}{c}=\beta \\
\sinh \theta & =\frac{\beta}{\sqrt{1-\beta^{2}}} \\
\cosh \theta & =\frac{1}{\sqrt{1-\beta^{2}}},
\end{aligned}
$$

con lo cual podemos escribir

$$
\begin{aligned}
c t^{\prime} & =\gamma(c t-\beta z) \\
x^{\prime} & =\gamma(x-\beta c t) \\
y^{\prime} & =y \\
z^{\prime} & =z
\end{aligned}
$$

donde $c$ es la velocidad de la luz, $\beta=v / c$ y $\gamma=\left(1-\beta^{2}\right)^{-1 / 2}$ el factor de Lorentz. Estas son un caso particular de las transformaciones de Lorentz. Éste es llamado un boost de Lorentz que puede ser considerado como una rotación hiperbólica.

El caso de un boost en una dirección general $\vec{v}$ de la velocidad relativa permite escribir las transformaciones de Lorentz como

$$
\begin{aligned}
c t^{\prime} & =\gamma\left(c t-\frac{\vec{v} \cdot \vec{x}}{c}\right) \\
\vec{x}^{\prime} & =\vec{x}+\frac{\gamma-1}{v^{2}}(\vec{v} \cdot \vec{x}) \vec{v}-\gamma \vec{v} t
\end{aligned}
$$

una manera abreviada de escribir la transformación es en una representación matricial

$$
x^{\prime \mu}=\Lambda_{\nu}^{\mu} x^{\nu}
$$

el conjunto de todas las posibles transformaciones es llamado grupo de Lorentz.

\section{B.2. Grupos de Lorentz}

Una transformación general entre $I^{\prime}$ y $I$ donde los orígenes coinciden en $x^{\prime 0}=x^{0}=0$, es una combinación de la rotación y un boost. Y es determinada por seis parámetros tres para la rotación y tres para los componentes de la velocidad. Tal transformación se puede escribir como

$$
x^{\prime \mu}=\Lambda_{\nu}^{\mu} x^{\nu}
$$

donde los elementos de la matriz $\Lambda^{\mu}{ }_{\nu}$ son reales y adimensionales. Las matrices $\Lambda^{\mu}{ }_{\nu}$ forman un grupo llamado grupo de Lorentz o grupo homogéneo de Lorentz $O(1,3)$. 
Físicamente podemos generalizar el grupo de Lorentz agregando una traslación a la transformación

$$
x^{\prime \mu}=\Lambda_{\nu}^{\mu} x^{\nu}+a^{\mu}
$$

esta es una transformación de Poincaré y el grupo de Lorentz se transforma en el grupo de Poincaré o grupo inhomogéneo de Lorentz, que tiene diez parámetros; tres para la orientación, tres para la velocidad y cuatro para la traslación; es decir el grupo de Lorentz, así como el grupo de las traslaciones son subgrupos del grupo de Poincaré.

Un grupo posee cuatro características básicas;

Definición. Sea $a, b$ y $c$ elementos del grupo, se debe cumplir la:

i) existencia de un elemento unidad $I: I a=a I=a$,

ii) propiedad de cerradura bajo la multiplicación de elementos del grupo: $a b=c$,

iii) ley asociativa bajo la multiplicación: $a b c=a(b c)=(a b) c$,

iv) existencia para cada elemento de un inverso $a^{-1}$, tal que: $a a^{-1}=a^{-1} a=I$.

De esta manera el grupo de Lorentz puede dividirse en un subgrupo y tres variedades. Como el determinante de $\Lambda$ es \pm 1 , podemos dividir el grupo inicialmente en dos conjuntos: $\operatorname{det} \Lambda=+1$, llamado de transformaciones propias; y det $\Lambda=-1$, de transformaciones impropias. También podemos considerar el valor del término $\Lambda_{0}^{0}$, que preserva o no la dirección: el caso $\Lambda_{0}^{0} \leqslant-1$ revierte la dirección del tiempo y $\Lambda_{0}^{0} \geqslant 1$, la preserva en cuyo caso la transformación se llama ortócrona y cada vector positivo tipo-tiempo lo transforma en otro positivo tipo-tiempo.

De esta manera los las cuatro partes son:

i) $L_{+}^{\uparrow}: \operatorname{det} \Lambda=+1, \Lambda_{0}^{0} \geqslant 1$, esta parte contiene la identidad $I$ del grupo, además de las transformaciones propias y ortócronas lo que forma un grupo, el grupo propio ortócrono de Lorentz.

ii) $L_{-}^{\uparrow}: \operatorname{det} \Lambda=-1, \Lambda_{0}^{0} \geqslant 1$, esta variedad contiene la inversión espacial, paridad $P$.

iii) $L_{-}^{\downarrow}: \operatorname{det} \Lambda=-1, \Lambda_{0}^{0} \leqslant-1$, esta variedad contiene la inversión temporal $T$.

iv) $L_{+}^{\downarrow}: \operatorname{det} \Lambda=+1, \Lambda_{0}^{0} \leqslant-1$, esta variedad contiene la combinación inversión temporal y paridad, reflexión fuerte $T P=J$.

De la unión de estas partes podemos obtener dos subgrupos el grupo de Lorentz ortócrono como $L^{\uparrow}=L_{+}^{\uparrow} \cup L_{-}^{\uparrow}$, y el grupo de Lorentz propio $L_{+}=L_{+}^{\uparrow} \cup L_{+}^{\downarrow}$. El grupo homogéneo de Lorentz puede reconstruirse como el producto semidirecto del grupo propio ortócrono de Lorentz y un elemento del subgrupo discreto $\{I, P, T, T P\}$. 



\section{Apéndice $C$}

\section{Método de Hirota}

Entre las distintas técnicas para construir solitones múltiples, quizá las más simples y directas son las transformaciones de Bäcklund y el método de Hirota. Las primeras fueron tratadas en capítulos previos y el último lo trataremos aquí.

Ryogo Hirota $(1971,1972)$ introdujo un nuevo método actualmente conocido como Método Directo de Hirota para encontrar la solución exacta de la ecuación KdV y sine-Gordon para colisiones múltiples de solitones; y en sucesivos trabajos lidió con las ecuaciones KdV modificada, Schrödinger no lineal y Toda lattice; dichos trabajos y el método se recopilan en su monografía Hirota (2004). Este método es de gran importancia en el estudio de sistemas integrables, ya que la mayoría de ecuaciones (incluso las no totalmente integrables) que pueden escribirse en forma bilineal o forma de Hirota, poseen solitones simples y dobles. Inclusive, esta condición no es suficiente para asegurar la integrabilidad de la ecuación, pero puede utilizarse como una herramienta para tal propósito. Sin embargo, la existencia de solitones dobles, no es trivial en general para cualquier forma bilineal.

El método directo suele usarse junto a la prueba de Painlevé como una prueba de integrabilidad, ya que es ampliamente creído y existen indicios de qué cualquier ecuación que posea solución 4-soliton es completamente integrable; vid. Newell (1985).

El método básicamente consiste de tres pasos:

1. Transformación: consiste en aplicar una transformación entre las variables adecuada para escribir la ecuación en una forma cuadrática de variables dependientes.

2. Bilinealización: escribe con ayuda de un nuevo operador $D$, un polinomio de dicho operador el cual es llamado forma bilineal de Hirota.

3. Expansión perturbacional: se realiza una expansión con un parámetro perturbacional y analiza los coeficientes del parámetro y sus potencias por separado. Lo que nos permite obtener una relación de dispersión para el solitón simple o múltiple. 
Como se ha mencionado el método directo puede aplicarse a una gran variedad de ecuaciones, en ocasiones la simplicidad del método depende de la transformación, ya que su elección puede llevar a una o varias ecuaciones bilineales. En algunos casos, se pueden obtener formas trilineales o múltilineales, de igual manera el método puede requerir la introducción de nuevas variables dependientes o independientes.

\section{C.1. Transformación}

La transformación que se aplica para cambiar la ecuación a una forma lineal, es en ocasiones un trabajo de prueba y error; existen sin embargo, una amplia búsqueda en esta dirección, v. g. Hietarinta (1987a,b,c, 1988). Una forma de lograr una transformación es partir de una solución conocida de la ecuación y construir una forma funcional general. Sin embargo, existen tres transformaciones fundamentales:

1. Transformación racional: es la transformación más fundamental tiene la forma $u=$ $f / g$ e introduce dos variables dependientes $f$ y $g$.

2. Transformación logarítmica: esta transformación $u=\alpha(\ln f)_{x x}$ a diferencia de la racional sólo introduce una variable dependiente pero introduce una constante.

3. Transformación bilogarítmica: como la racional introduce dos variables dependientes $f$ y $g$, y puede utilizarse ya sea como un cociente o un producto $u=\alpha \ln (f / g)$ o $u=\alpha \ln (f g)$.

\section{C.2. Bilinealización}

Junto con la transformación se define un nuevo operador diferencial binario $D$, llamado en ocasiones derivada de Hirota.

Definición. Se define el operador $D$ que actúa sobre dos funciones $a(t, x)$ y $b(t, x)$

$$
D_{t}^{m} D_{x}^{n} a(t, x) \cdot b(t, x):=\left.\frac{\partial^{m}}{\partial s^{m}} \frac{\partial^{n}}{\partial r^{n}} a(t+s, x+r) b(t-s, x-r)\right|_{s=0, r=0},
$$

donde $m, n=0,1,2,3, \ldots$ Para obtener algunas propiedades e identidades el operador $D$ puede escribirse usando funciones exponenciales de la siguiente forma

$$
\exp \left(\delta D_{z}\right) a(z) \cdot b(z)=a(z+\delta) b(z-\delta)
$$

donde $\delta$ es un parámetro. 
El operador $D$ puede comparase con la regla de Leibniz para la derivada estándar, escribiendo

$$
\frac{\partial^{m}}{\partial t^{m}} \frac{\partial^{n}}{\partial x^{n}} a(t, x) \cdot b(t, x)=\left.\frac{\partial^{m}}{\partial s^{m}} \frac{\partial^{n}}{\partial r^{n}} a(t+s, x+r) b(t+s, x+r)\right|_{s=0, r=0}
$$

donde hay que notar la diferencia en signo.

Entre las relaciones que se pueden obtener para este operador se encuentra la identidad de Jacobi

$$
D_{z}\left(D_{z} a \cdot b\right) \cdot c+D_{z}\left(D_{z} b \cdot c\right) \cdot a+D_{z}\left(D_{z} c \cdot a\right) \cdot b=0
$$

la cual al escribir $D_{z} a \cdot b=[a, b]$, se obtiene

$$
[[a, b], c]+[[b, c], a]+[[c, a], b]=0
$$

lo que indica una conexión entre el operador $D$ y las álgebras de Lie. Otra relación importante, es que la forma bilineal de Hirota obtenida es invariante de norma.

Al reescribir la ecuación en términos de las derivadas de Hirota y simplificar se pueden escribir uno o más polinomios del operador $D$, y ayudándonos en ocasiones de funciones arbitrarias, resultando en la llamada forma bilineal de Hirota. Es importante notar que el método dista de ser algorítmico, debido a la elección del la transformación; de hecho algunas ecuaciones no tendrán forma bilineal, pero quizá trilineal. Incluso la cantidad de funciones dependientes y variables independientes que se introducen puede ser muy variable.

\section{C.3. Expansión perturbacional}

Para obtener las soluciones solitónicas de la forma bilineal se debe hacer una expansión finita alrededor de algún vacío constate $f_{0}$ y $g_{0}$ de la funciones dependientes utilizando un parámetro perturbacional $\epsilon$, las funciones $f_{i}$ y $g_{i}$ con $i=1,2,3, \ldots$ son funciones de las variables independientes

$$
\begin{aligned}
& f=\epsilon^{0} f_{0}+\epsilon f_{1}+\epsilon^{2} f_{2}+\epsilon^{3} f_{3}+\cdots \\
& g=\epsilon^{0} g_{0}+\epsilon g_{1}+\epsilon^{2} g_{2}+\epsilon^{3} g_{3}+\cdots
\end{aligned}
$$

y substituirlo en la forma bilineal. Es importante notar que la expansión perturbacional finita para el caso de una ecuación bilineal nos da una solución exacta no una aproximación. 
Realizando el producto de las expansiones

$$
\begin{aligned}
f \cdot g= & \epsilon^{0}\left(f_{0} \cdot g_{0}\right) \\
& +\epsilon^{1}\left(f_{0} \cdot g_{1}+f_{1} \cdot g_{0}\right) \\
& +\epsilon^{2}\left(f_{0} \cdot g_{2}+f_{1} \cdot g_{1}+f_{2} \cdot g_{0}\right) \\
& +\epsilon^{3}\left(f_{0} \cdot g_{3}+f_{1} \cdot g_{2}+f_{2} \cdot g_{1}+f_{3} \cdot g_{0}\right)+\cdots
\end{aligned}
$$

sustituyendo en la forma bilineal y agrupando las potencias de $\epsilon$

$$
\begin{aligned}
P(D) f \cdot g= & \epsilon^{0} P(D)\left(f_{0} \cdot g_{0}\right) \\
& +\epsilon^{1} P(D)\left(f_{0} \cdot g_{1}+f_{1} \cdot g_{0}\right) \\
& +\epsilon^{2} P(D)\left(f_{0} \cdot g_{2}+f_{1} \cdot g_{1}+f_{2} \cdot g_{0}\right) \\
& +\epsilon^{3} P(D)\left(f_{0} \cdot g_{3}+f_{1} \cdot g_{2}+f_{2} \cdot g_{1}+f_{3} \cdot g_{0}\right)+\cdots
\end{aligned}
$$

donde $P(D)$ es el polinomio de la forma bilineal de la ecuación.

Veamos algunos de estos términos de la expansión

$$
\begin{aligned}
P(D)\left(f_{0} \cdot g_{0}\right) & =0 \\
P(D)\left(f_{i} \cdot g_{0}\right) & =g_{0} P(\partial) f_{i} \\
P(D)\left(f_{0} \cdot g_{i}\right) & =f_{0} P(-\partial) g_{i}
\end{aligned}
$$

estos términos son los que involucran el orden cero de las series.

Con el fin de obtener soluciones solitónicas las formas bilineales deben igualarse a cero. Si la solución solitónica existe, las series se truncarán y la serie finita conducirá a una solución exacta.

\section{C.3.1. Solución solitón simple}

Para obtener la solución simple del solitón se considera el primer orden de $\epsilon$ de la expansión y podemos escribir los demás términos consideremos $f_{1}$ y $g_{1}$ de la forma

$$
h_{1}=H_{1} \exp \eta_{1}
$$

con $\eta_{1}=\kappa_{1} x+\Omega_{1} t+\alpha_{1}$, donde $\kappa_{1}, \Omega_{1}, \alpha_{1}$, y $H_{1}$ son constantes al utilizar estos términos en la expresión de orden- $\epsilon^{2}$ podemos elegir $h_{2}=0$; con lo que podemos truncar la serie. En esta expresión, $P(\partial)\left(\eta_{i}\right)=0$ forman las llamadas relaciones de dispersión no lineales; y los coeficientes $H_{i}$ se forman de las constantes $\kappa_{j}$ y $\Omega_{j}$.

Para el tipo de ecuación que tenemos consideremos hasta un orden mayor, es decir, $h_{2} \neq 0$; vid. Alagesan et al. (2004). 


\section{C.3.2. Solución solitón doble}

Para obtener la solución doble del solitón, considerando el primer orden de $\epsilon$ de la expansión podemos escribir los demás términos consideremos $f_{i}$ y $g_{j}$ de las formas

$$
\begin{aligned}
& h_{1}=H_{1} \exp \eta_{1}+H_{2} \exp \eta_{2} \\
& h_{2}=H_{12} \exp \left(\eta_{1}+\eta_{2}\right)
\end{aligned}
$$

con $\eta_{k}=\kappa_{k} x+\Omega_{k} t+\alpha_{k}$, donde $\kappa_{k}, \Omega_{k}, \alpha_{k}$, y $H_{k}$ son constantes. E introduciendo las $h_{k}$ en la expresión de $\epsilon^{2}$ se encuentra que $H_{12}$ es función de las constantes $\kappa_{k}$ y $\Omega_{k}$, y ésta representa un corrimiento de fase debido a la interacción de las ondas. Ahora substituyendo las formas $h_{1}$ y $h_{2}$ en la expresión de orden $\epsilon^{3}$, permite elegir $h_{3}=0$.

Consideremos el tipo de expresiones que se obtienen al considerar las formas de $h_{k}$ propuestas anteriormente; para formas

$$
\begin{aligned}
h_{k} & =H_{k} \exp \eta_{k} \\
h_{k} & =H_{k} \exp \eta_{k}+H_{k+1} \exp \eta_{k+1} \\
h_{i j} & =H_{i j} \exp \left(\eta_{i}+\eta_{j}\right)
\end{aligned}
$$

introduciendo esto en los polinomios

$$
\begin{gathered}
P(D)\left(f_{i} \cdot g_{j}\right)=P(D)\left(\left(F_{i} \exp \phi_{i}+F_{i+1} \exp \phi_{i+1}\right) \cdot\left(G_{j} \exp \varphi_{j}\right)\right) \\
=\quad F_{i} G_{j} P(D)\left(\exp \phi_{i} \cdot \exp \varphi_{j}\right) \\
+F_{i+1} G_{j} P(D)\left(\exp \phi_{i+1} \cdot \exp \varphi_{j}\right) \\
P(D)\left(f_{k} \cdot h_{i j}\right)=P(D)\left(F_{k} \exp \phi_{k} \cdot H_{i j} \exp \left(\eta_{i}+\eta_{j}\right)\right) \\
=F_{k} H_{i j} P(D)\left(\exp \phi_{k} \cdot \exp \left(\eta_{i}+\eta_{j}\right)\right)
\end{gathered}
$$

ambas formas se reducen al cálculo del polinomio de exponenciales, si los polinomios son de la la forma $D_{x}^{m} D_{t}^{n}$ obtenemos

$$
\begin{aligned}
D_{x}^{m} D_{t}^{n}\left(\exp \phi_{i} \cdot \exp \varphi_{j}\right) & =\left(\phi_{i, x}-\varphi_{j, x}\right)^{m}\left(\phi_{i, t}-\varphi_{j, t}\right)^{n} \exp \left(\phi_{i}+\varphi_{j}\right) \\
& =\left(\kappa_{i}-\kappa_{j}\right)^{m}\left(\Omega_{i}-\Omega_{j}\right)^{n} \exp \left(\phi_{i}+\varphi_{j}\right)
\end{aligned}
$$

las constantes $\kappa_{k}, \Omega_{k}$ junto con los coeficientes $H_{k}$ forman los coeficientes de la solución. 



\title{
Solitonic axion condensates modeling dark matter halos
}

\author{
David Castañeda Valle, Eckehard W. Mielke* \\ Departamento de Física, Universidad Autónoma Metropolitana Iztapalapa, Apartado Postal 55-534, \\ C.P. 09340, México, D.F., Mexico
}

\section{H I G H L I G H T S}

- An axion model of dark matter is considered.

- Collision of axion type solitons are studied in a two dimensional toy model.

- Relations to dark matter collisions in galaxy clusters are proposed.

\section{A R T I C L E I N F O}

\section{Article history:}

Received 4 October 2012

Accepted 17 May 2013

Available online 13 June 2013

\section{Keywords:}

Dark matter

Universal axion

Soliton

Dark matter halo

Galaxy cluster

\begin{abstract}
A B S T R A C T
Instead of fluid type dark matter (DM), axion-like scalar fields with a periodic self-interaction or some truncations of it are analyzed as a model of galaxy halos. It is probed if such cold Bose-Einstein type condensates could provide a viable soliton type interpretation of the DM 'bullets' observed by means of gravitational lensing in merging galaxy clusters. We study solitary waves for two self-interacting potentials in the relativistic Klein-Gordon equation, mainly in lower dimensions, and visualize the approximately shape-invariant collisions of two 'lump' type solitons.

(c) 2013 Elsevier Inc. All rights reserved.
\end{abstract}

\section{Introduction}

Non-interacting cold dark matter (CDM) is the standard model in order to account for the 'missing matter' of Zwicky [1] in galactic halos and cumulus of galaxies. However, numerical simulations quite generally run into a 'cusp' in the density of the central core which is in conflict with observations of low-surface brightness (LSB) galaxies.

Therefore, and in view of the almost confirmed Higgs boson [2] in the standard model of particle interactions, the DM fluid has been tentatively [3] substituted by a very light self-interacting scalar

\footnotetext{
* Corresponding author.

E-mail addresses: casvada@gmail.com (D. Castañeda Valle), ekke@xanum.uam.mx (E.W. Mielke).
} 
field. The case of a $\phi^{6}$ potential is particularly instructive, as it leads, for spherically symmetric configurations, to an exactly solvable Lane-Emden equation [4] with reasonable rotation curves [5]. Moreover, scaling relations [6] for the central densities quite well fit observations and, to some extent, galactic substructures as well as angular momentum $[7,8]$ can be accommodated. More recently, we have shown that the universal axion of quantum chromodynamics (QCD), a prime DM candidate, with its periodic effective potential provides Bessel type spherically symmetric configurations [9] and reasonable rotation curves. So far, these soliton-type configurations, in 3D behaving more like lumps and not like domain walls, are static.

Head-on collisions of massive galaxy clusters, like those occurring in the so-called bullet cluster [10] as well as the gigantic merger Abell 520 containing a dark matter core [11], could provide a dynamical test. They seem to defy explanation within the CDM paradigm, inasmuch either the individual galaxies or the hot X-ray emitting interstellar gas are not anchored to the DM lumps. Therefore, we are going to probe if colliding DM halos can be modeled via soliton-type collisions.

Solitary waves have been studied since the legendary discovery in a narrow water channel by John Scott Russell in August of 1834. However, they have been ignored for quite some time until in 1965 Zabusky and Kruskal [12] could describe its mathematical structure and corroborated the reconstruction of the initial state during nonlinear interactions. It turned out that every soliton is a localized solitary wave, but not all localized waves behave like solitons being exactly shape-invariant after collisions.

Among nonlinear differential equations exhibiting soliton type solutions are the well-known Korteweg-de Vries (KdV) equation in two dimensions (2D), those of Kadomtsev-Petviashvili (KP) which supply solitons in three dimensions, the nonlinear Schrödinger (NLS) equation, that of Boussinesq for shallow water waves, the nonlinear Klein-Gordon (NLKG) equation, and the sine-Gordon (sG) equation in 2D. The latter occur in various phenomena, like crystal dislocations, splay waves in nematic liquid crystals, transmission lines, etc.; cf. Refs. [13-18]. Further applications arise in 2D Josephson junctions [19], in hydrodynamics, including giant water waves (tsunamis), shock waves in the earth crust, protein chains, nonlinear optics, plasmas, effectively nonlinear Dirac equations [20] as well as vortices occurring in the Great Red Spot of Jupiter [21].

In astrophysics they have been analyzed in order to describe compact objects like the hypothetical boson stars (BS). The latter behave like Bose-Einstein condensates (BEC) on a macroscopic scale, where the self-generated gravitational fields provides a 'trap' for the scalar field; cf. Ref. [22-24]. In our scenario, solitons built from pseudo-scalars like the axion of QCD [25] could serve as a model [26, $9,27,28]$ for dark matter halos. Consequently, we will depart from the sine-Gordon equation and its Lane-Emden truncation in 2D as a toy model describing semi-classically dark matter solitons.

Our paper is organized as follows. In the next section, the formal framework of nonlinear KG equations together with its reduction to the Bogomol'nyi equation in 2D spacetime dimension is set-up. The case of the periodic axion potential, its relation to the sine-Gordon equation as well as the corresponding superpotentials are recapitulated in Section 3, again in 2D. Its higher order truncation, the Lane-Emden model together with the energy content of its lump type solutions, is studied in Section 4. In order to analyze soliton collisions, we review in Section 5 relevant aspects of a kind of 'nonlinear' superposition, the Bäcklund transformations, a standard tool in the case of the $\mathrm{SG}$ equation. However, the Lane-Emden truncation is not completely integrable, so we have to resort to a new approximate method via a mapping to the sine-Gordon case. Then a colliding solution for two lumps results which, to our knowledge, is completely new. In an Outlook, we speculate on a possible resemblance of such a soliton scattering with the observed DM collisions in the bullet and Abell cluster and remark on the necessary generalization to (head-on) collisions of the corresponding three-dimensional (3D) solitary waves.

\section{Nonlinear Klein-Gordon equation}

Let $\square:=\nabla \bullet \nabla-\partial^{2} / c^{2} \partial t^{2}$ be the wave operator of Jean le Rond d'Alembert and $c$ the velocity of light in vacuum. Then

$$
\square \phi=\frac{\partial V(\phi)}{\partial \phi},
$$


is the relativistically invariant Klein-Gordon (KG) equation for a single scalar field $\phi=\phi(t, \vec{x})$ with a self-interaction potential $V(\phi)$. In its series expansion, the quadratic term returns the mass term in the original KG equation, whereas higher order terms account for a possible nonlinearity.

Such equations are invariant under the discrete symmetries $P$ and $T$ of parity and time-reflection. Moreover, they are invariant under the standard Lorentz transformations such that one can resolve the equation in a rest frame where a static solution $\phi=\phi(\vec{x})$ depends only on the spatial coordinates $\vec{x}$. In order to get a traveling wave solution or 'moving soliton', one has to apply a Lorentz boost

$$
\vec{x} \rightarrow \gamma(\vec{x}-\vec{u} t), \quad \gamma=1 / \sqrt{1-\vec{u} \bullet \vec{u} / c^{2}}
$$

where $\gamma$ is the Lorentz factor and $\vec{u}$ the (phase) velocity of the solitary wave. Then one can recover a stationary solution in an inertial frame moving with velocity $\vec{u}$ relative to a rest frame.

\subsection{Superpotential and Bogomol'nyi equation in $(1+1) \mathrm{D}$}

In a two-dimensional (2D) toy model, we consider first static, i.e. time-independent solution of a nonlinear KG equation. Then Eq. (1) reduces to

$$
\frac{\partial^{2} \phi}{\partial x^{2}}=\frac{\partial V(\phi)}{\partial \phi} .
$$

After multiplying both sides by $2 \phi^{\prime}$, the first integral turns out to be the Bogomol'nyi equation

$$
\frac{\partial \phi}{\partial x}= \pm \sqrt{2 V(\phi)}
$$

of first differential order; cf. Refs. [29-32]. In the case that the potential $V(\phi)$ arises from a so-called superpotential $W(\phi)$ implicitly defined by

$$
W_{\phi}^{2}:=\left(\frac{d W}{d \phi}\right)^{2}=2 V(\phi),
$$

the Bogomol'nyi equation can be rewritten as follows:

$$
\frac{d \phi}{d x}=W_{\phi}, \quad \frac{d \phi}{d x}=-W_{\phi} .
$$

Since the fields are assumed to be static in the rest frame, i.e. $\partial \phi / \partial t=0$, the rest energy of the soliton simply follows by a further integration as

$$
\begin{aligned}
E_{0}: & =\int\left[\frac{1}{2}\left(\frac{d \phi}{d x}\right)^{2}+V(\phi)\right] c^{2} d x \\
& =\int\left(\frac{d W}{d \phi}\right)\left(\frac{d \phi}{d x}\right) c^{2} d x \\
& =\left|W\left(\phi_{+}\right)-W\left(\phi_{-}\right)\right| c^{2} .
\end{aligned}
$$

Here $\phi_{ \pm}=\phi(x= \pm \infty)$ are the respective boundary values at infinity.

Solitons connecting two minima are fixing a topological sector such that a topological charge $Q$ can be defined via integrating the temporal component of the current

$$
J^{\mu}:=\frac{1}{L} \epsilon^{\mu \nu} \partial_{\nu} \phi, \quad \partial_{\mu} J^{\mu} \equiv 0
$$

being conserved due to the Poincaré lemma for smooth fields. The 'topological width' $L$ is a normalization constant. 


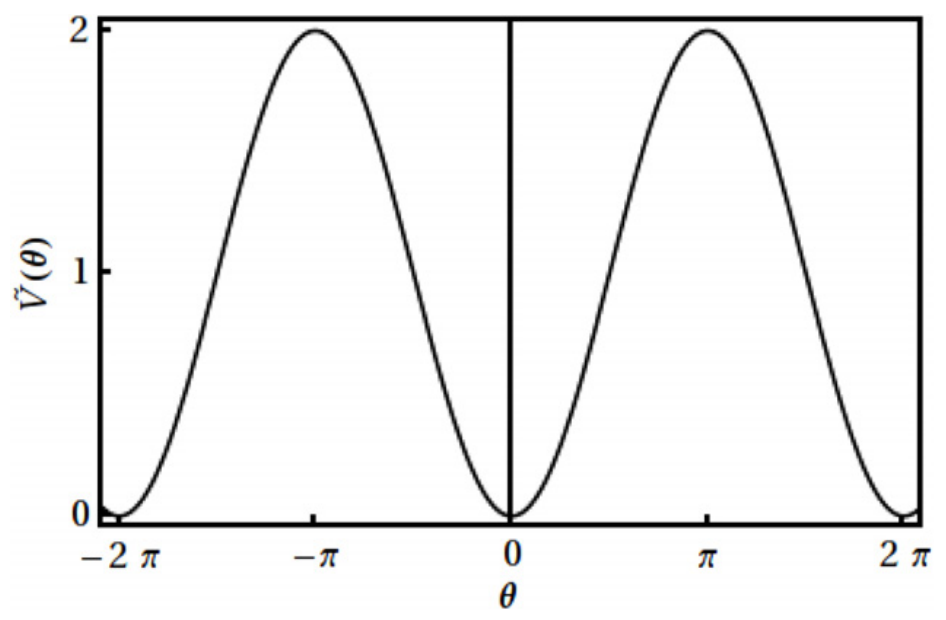

Fig. 1. Periodic axion potential $\tilde{V}(\theta)=1-\cos \theta$.

\section{Axion potential}

In QCD, the axion of inertial mass $m$ is self-interacting via the effective potential

$$
\begin{aligned}
V(\phi) & =\frac{m^{4}}{\lambda}\left[1-\cos \left(\frac{\sqrt{\lambda}}{m} \phi\right)\right] \\
& \simeq \frac{m^{2}}{2} \phi^{2}-\frac{\lambda}{4 !} \phi^{4}+\frac{\lambda^{2}}{6 ! m^{2}} \phi^{6}-\cdots
\end{aligned}
$$

arising from the chiral anomaly after integrating out the gluon field; cf. Ref. [25] for details. For a very light pseudo-scalar, it is almost flat at the origin. In 2D, it implies the sine-Gordon equation

$$
\square \phi-\frac{m^{3}}{\sqrt{\lambda}} \sin \left(\frac{\sqrt{\lambda}}{m} \phi\right)=0,
$$

considered already in 1862 by Bour as a compatibility conditions for pseudospherical surfaces; cf. Ref. [33].

Rescaling variables [14] via

$$
\tilde{x}=m x, \quad \tilde{t}=m t, \quad \tilde{\phi}=\frac{\sqrt{\lambda}}{m} \phi=\theta,
$$

allows us to simplify the $\mathrm{sG}$ equation to

$$
\tilde{\square} \theta=\sin \theta \text {. }
$$

This equation is invariant under $\theta(\tilde{x}, \tilde{t}) \rightarrow-\theta(\tilde{x}, \tilde{t})$ which can be associated with charge conjugation $C$. The potential has zeros and critical points defined by the conditions (see Fig. 1)

$$
\widetilde{V}(\theta):=\frac{\lambda}{m^{4}} V(\phi)=1-\cos \theta=0
$$

and

$$
\frac{d}{d \theta} \widetilde{V}(\theta)=\sin \theta=0 .
$$

Thus, there exist infinite many critical points at $\overline{\theta_{n}}=2 n \pi$, with equal spacing due to $n \in \mathbb{Z}$. In this case, solutions can interpolate critical points, and in such a topological sector of width $2 \pi$, the boundary conditions are $\tilde{x} \rightarrow-\infty$ and $\tilde{x} \rightarrow+\infty$. In view of Eq. (8) the topological charge is simply given by

$$
Q=\int J^{0} d \tilde{x}=\frac{1}{2 \pi}\left[\theta_{+}-\theta_{-}\right]
$$




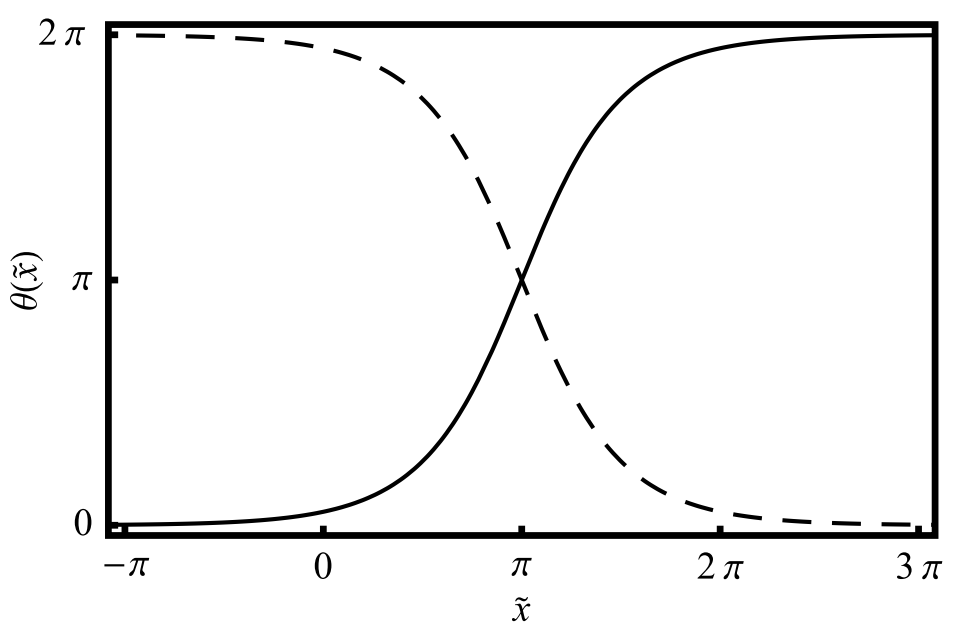

Fig. 2. Kink and antikink solutions centered at $\tilde{x}_{0}=\pi$.

The shape of solitary solutions of the sG equation can be obtained by solving first the static equation

$$
\frac{\partial^{2} \theta}{\partial \tilde{x}^{2}}=\sin \theta, \quad \bmod 2 \pi
$$

Since only two minima with topological charge $Q= \pm 1$ can be connected, the resulting solitons are of kink y antikink type, corresponding to positive and negative charges, respectively.

The periodicity of the axion potential implies the existence of solutions centered at different $x_{0}$ 's, depending on the critical points considered, each giving rise to a kink or antikink. Then, the Bogomol'nyi equation reads

$$
\frac{d \theta}{d \tilde{x}}= \pm \sqrt{2(1-\cos \theta)}= \pm 2 \sin \frac{\theta}{2} .
$$

Its implicit solution

$$
\tilde{x}-\tilde{x}_{0}= \pm \ln \left|\tan \frac{\theta(\tilde{x})}{4}\right|
$$

can be inverted for the axion type field

$$
\theta(\tilde{x})=4 \arctan \left\{\exp \left[ \pm\left(\tilde{x}-\tilde{x}_{0}\right)\right]\right\} .
$$

These kink and antikink type solutions drawn in Fig. 2 are, later on, denoted by $\mathrm{k}$ and $\overline{\mathrm{k}}$, respectively. The positive or negative sign $( \pm)$, respectively, associated with the topological charge, determines the chirality with which the waves propagate $[13,14]$. One identifies the positive sign with a soliton or kink (Fig. 2(a)), and the negative sign with an antisoliton or antikink (Fig. 2(b)), in accordance with the symmetry of charge conjugation $C$ of the field within adjacent sectors of the topology.

In the case of solitons of the kink type, the critical values increment from $\bar{\theta}_{c}=2 \pi N$ to $\bar{\theta}_{c}=$ $2 \pi(N+1)$ resulting in the charge $Q=+1$, while for antikinks, the values reduce from $\bar{\theta}_{\mathrm{c}}=2 \pi N$ to $\bar{\theta}_{\mathrm{c}}=2 \pi(N-1)$ leading to the charge $Q=-1$.

When applying a Lorentz boost to Eq. (19), we obtain the stationary solution

$$
\theta(\tilde{x}, \tilde{t})=4 \arctan \left[\exp \left( \pm \gamma\left(\tilde{x}-\tilde{x}_{0}-u \tilde{t}\right)\right)\right]
$$

An integration leads to the defining equation

$$
\frac{d \widetilde{W}}{d \theta}= \pm 2 \sin \frac{\theta}{2}
$$




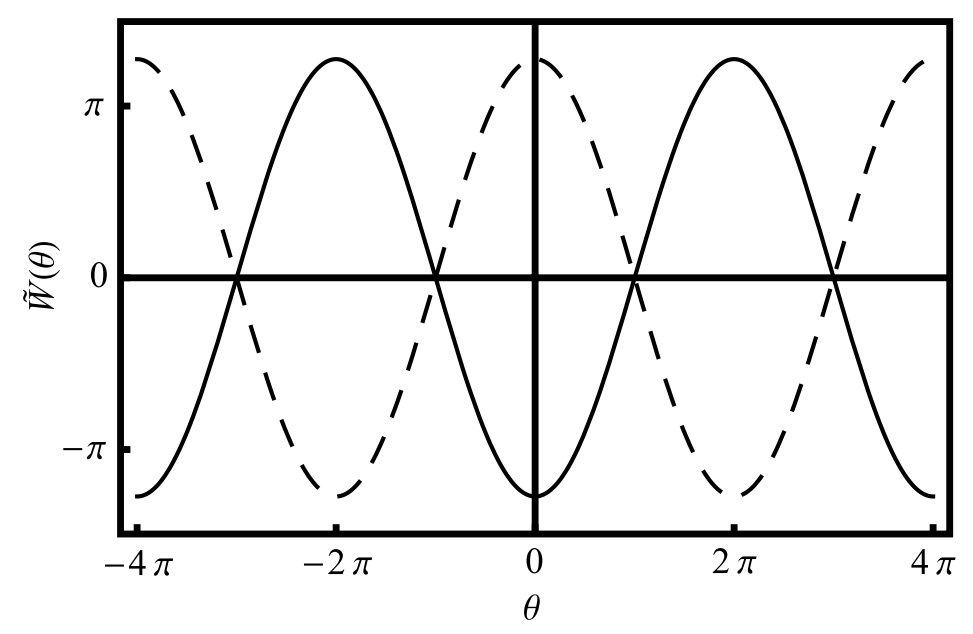

Fig. 3. The axionic superpotentials of a kink and antikink, respectively.

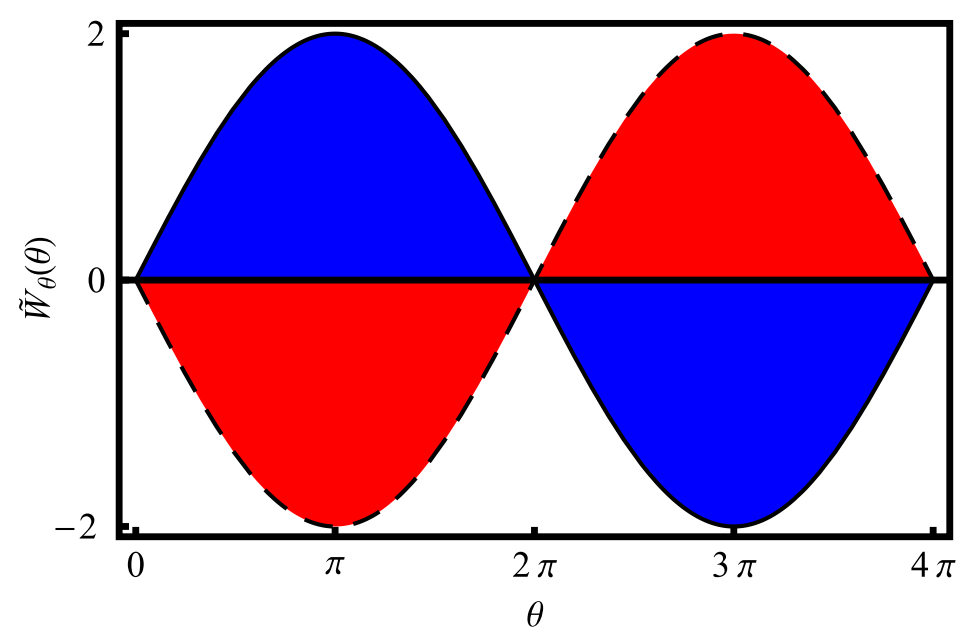

Fig. 4. (Color online) Gradient of the superpotential determining the energy of a kink and an antikink, respectively.

for the rescaled superpotential. It turns out that

$$
\widetilde{W}_{\mathrm{k}, \overline{\mathrm{k}}}(\theta)=\mp 4 \cos \frac{\theta}{2}
$$

for kink or antikink solutions again are periodic; cf. Fig. 3.

According to (7), these superpotentials and there derivatives (Fig. 4) facilitate the calculation of the energy of a kink or antikink, respectively:

$$
\begin{aligned}
E_{0} & =\left|W_{\mathrm{k}}\left(\bar{\theta}_{n}(-\infty)\right)-W_{\mathrm{k}}\left(\bar{\theta}_{n+1}(\infty)\right)\right| c^{2} \\
& =\left|W_{\overline{\mathrm{k}}}\left(\bar{\theta}_{n+1}(-\infty)\right)-W_{\overline{\mathrm{k}}}\left(\bar{\theta}_{n}(\infty)\right)\right| c^{2} \\
& =|W(2 n \pi)-W(2(n+1) \pi)| c^{2}=8 \frac{m^{3}}{\lambda} c^{2} .
\end{aligned}
$$

Since the rest energy is $E_{0 \mathrm{k}}=m_{\mathrm{k}} c^{2}$, we can identify the effective mass of the kink as $m_{\mathrm{k}}=8 \mathrm{~m}^{3} / \lambda$. Applying the Lorentz factor $\gamma$, the relativistic energy of a moving kink is, according to Einstein, given by $E=\gamma m_{\mathrm{k}} c^{2}$ and its momentum by $p_{\mathrm{k}}=\gamma m_{\mathrm{k}} u$. 


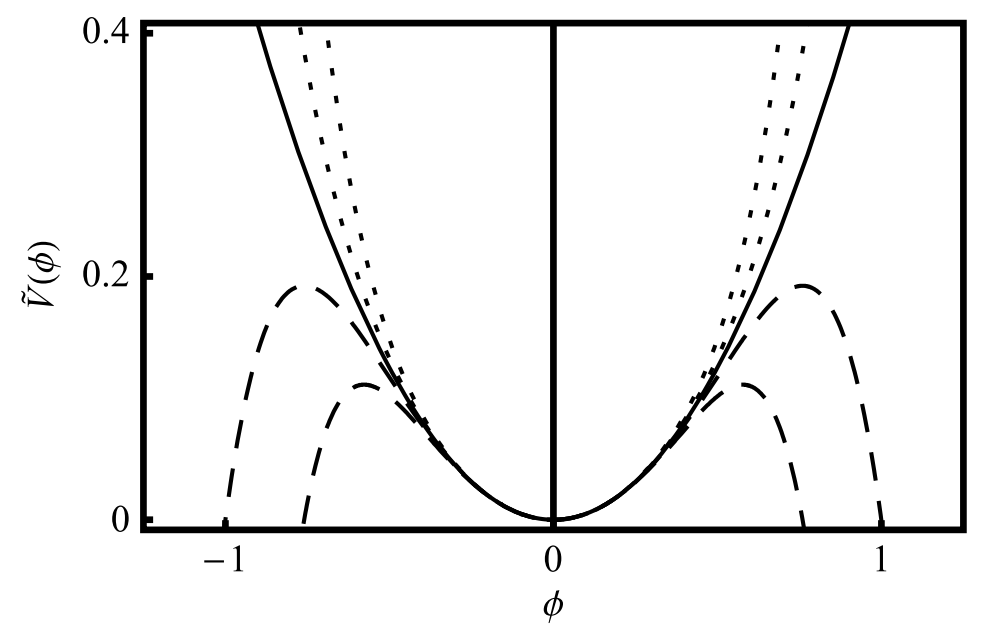

Fig. 5. Lane-Emden potential $\tilde{V}(\phi)=\phi^{2}\left(1-\chi \phi^{4}\right) / 2$. For $\chi=0$, it reduces to quadratic potential (solid line); when $\chi<0$ there arise parabolic curves (punctuated lines), whereas for $\chi>0$ the potential has two maxima and one local minimum (dashed lines).

\section{Lane-Emden potential}

In the astrophysics of gaseous spheres [34-36], like that of the Sun, the density has been modeled, for quite some time, by a potential of the $\phi^{6}$ type. Moreover, such a potential is mathematically convenient for modeling galaxy halos, since the Lane-Emden equation in $(1+3) \mathrm{D}$ is exactly solvable [5,7] in spherical coordinates.

In applying this to solitary waves, let us depart from the symmetric potential

$$
V(\phi)=\frac{m^{2}}{2} \phi^{2}\left(1-\chi \phi^{4}\right)=V(-\phi),
$$

where $m$ is a mass like parameter and $\chi$ a coupling constant for the nonlinear self-interaction; cf. Fig. 5. It can be regarded as a truncation of the axion potential (9), but this time of a particular higher order.

The form of the potential depends essentially on the coupling constant $\chi$. For $\chi \leq 0$ there is only one local minimum, whereas for $\chi>0$ there are, additionally, two local maxima, all vacuum states. Obviously, for $\chi=0$ the dynamics reduces to the linear Klein-Gordon equation, whereas $\chi<0$ would imply imaginary mass terms similarly as in the celebrated Higgs mechanism. Here, we will restrict ourselves to the case $\chi>0$. Although then the potential gets unbounded, even in 3D it admits meta-stable $[37,4]$ radial configurations.

Then the static KG equations in $2 \mathrm{D}$ takes the form

$$
\frac{\partial^{2} \phi}{\partial \tilde{x}^{2}}=\phi\left(1-3 \chi \phi^{4}\right)
$$

which may lead, at least, to two types of solitonic solutions: non-topological lump type solutions as well as topological ones such as kinks and antikinks [38].

\subsection{Lump type solutions}

The zeros $\phi_{0}^{2}=0$ and $\phi_{0}^{4}=1 / \chi$ of the potential (24) are degenerate, whereas the critical points are occurring for $\phi_{\mathrm{c}}=0$ and $\phi_{\mathrm{c}}^{4}=1 / 3 \chi$. Thus, there is only the point $\phi=0$ which minimize the energy implying that the corresponding solution is non-topological of lump type centered at $\tilde{x}_{0}$. The Bogomol'nyi equation now reads

$$
\frac{d \phi}{d \tilde{x}}= \pm \phi \sqrt{1-\chi \phi^{4}}
$$


Since the potential is symmetric under reflection, there will be two solutions, one for $\phi>0$ and another for $\phi<0$. Moreover, in order that the integral remains real, we require that $\chi \phi^{4} \leq 1$. Then

$$
\tilde{x}-\tilde{x}_{0}=\mp \int_{\phi\left(\tilde{x}_{0}\right)}^{\phi(\tilde{x})} \frac{d \phi}{\phi \sqrt{1-\chi \phi^{4}}}
$$

and an inversion leads to the explicit solutions

$$
\phi(\tilde{x})= \pm \chi^{-1 / 4}\left[\operatorname{sech}\left(2\left(\tilde{x}-\tilde{x}_{0}\right)\right)\right]^{1 / 2},
$$

again one in the case that $\phi>0$ and another for $\phi<0$; cf. Fig. 6 .

Now we integrate once more the differential condition

$$
\frac{d \widetilde{W}}{d \phi}= \pm \phi \sqrt{1-\chi \phi^{4}}
$$

in order to obtain the superpotential

$$
\widetilde{W}(\phi)=\mp \frac{1}{4}\left(\frac{\arcsin \left(\chi^{1 / 2} \phi^{2}\right)}{\chi^{1 / 2}}+\phi^{2} \sqrt{1-\chi \phi^{4}}\right) ;
$$

cf. Fig. 7.

Depending on the range $\left[0, \pm 1 / \chi^{1 / 4}\right]$, the solution $\phi(\tilde{x})$ is positive or negative. Therefore, the rest energy or effective mass turns out to be

$$
\begin{aligned}
E_{\text {lump }} / c^{2} & =\left|W\left(\phi_{-}\right)-W\left(\phi_{0}\right)\right|+\left|W\left(\phi_{0}\right)-W\left(\phi_{+}\right)\right| \\
& =2\left|W\left(\phi_{0}\right)-W\left(\phi_{+}\right)\right| \\
& =2\left|W\left(\chi^{-1 / 4}\right)-W(0)\right|=\frac{\pi}{4} \frac{m}{\chi^{1 / 2}}
\end{aligned}
$$

cf. Fig. 8.

\section{Soliton collisions}

When two or more solitons collide, we expect that they recuperate their initial shapes and velocities after some (infinity) time has passed [12]. Remnants from crossing the scattering region are the concomitant phase shifts in the individual solitary waves.

The most common techniques for constructing multi-solitons are the inverse scattering transform (IST) of Gardner et al. [39] and the Bäcklund transformation (BT) which are both connected via the Riccati equation, see Ref. [33], emphasizing the geometrical origins.

While IST is a rather general method for generating multi-solitons, BTs are simpler for obtaining the desired solutions of collisions and, moreover, are able to bridge a gap between different types of nonlinear equations. Moreover, in some cases of interest here, the latter permits a purely algebraic construction, whereas IST requires not only the solution to an initial value problem, but also that of an integral equation, the so-called Gel'fand-Levitan-Marchenko equation.

For the Lane-Emden equation of interest here, it was not possible to define an exact auto-Bäcklund transformation. Instead a generalized transformation will serve us as a guide to check the validity of our approximations.

\subsection{Bäcklund transformations}

Let us reconsider the Lane-Emden potential (24). Then the corresponding NLKG equation adopts the form

$$
\left(\frac{\partial^{2}}{\partial \tilde{x}^{2}}-\frac{1}{c^{2}} \frac{\partial^{2}}{\partial \tilde{t}^{2}}\right) \phi=\phi\left(1-3 \chi \phi^{4}\right)
$$




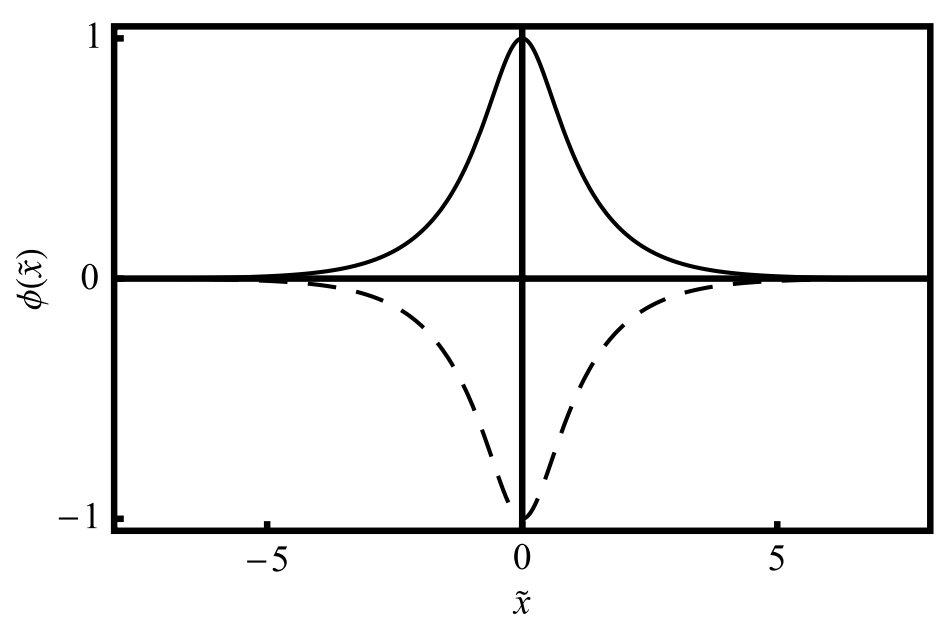

Fig. 6. Lump and antilump (dashed line) Lane-Emden solutions centered at $\tilde{x}_{0}=0$.

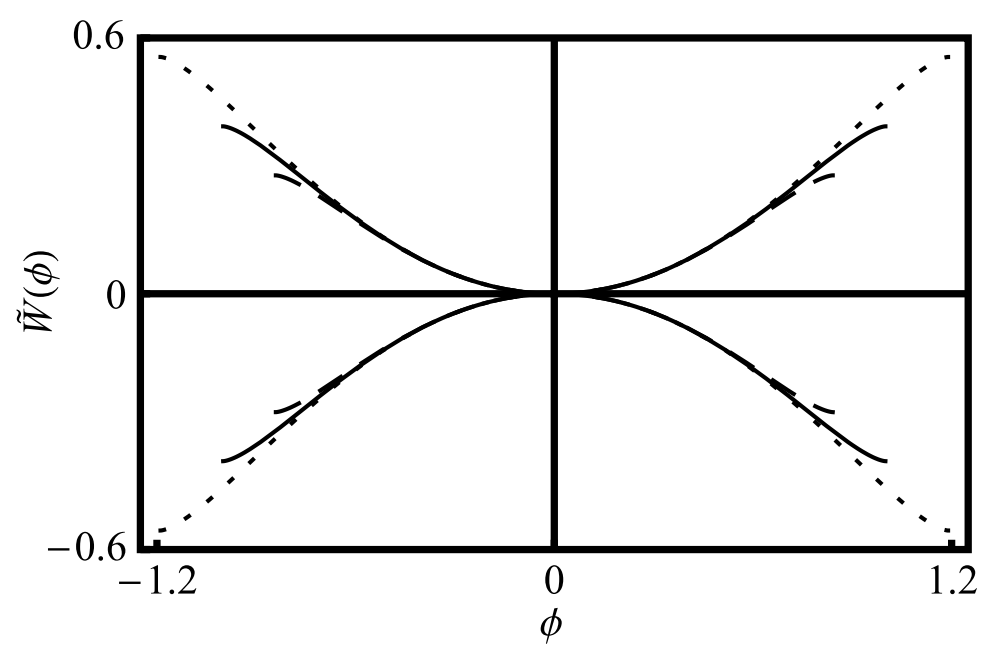

Fig. 7. Superpotentials for the cases $\phi>0$ and $\phi<0$. For $\chi=0.5$ they are presented by a punctuated line, in the case $\chi=1$ by solid line and for $\chi=2$ by a dashed line.

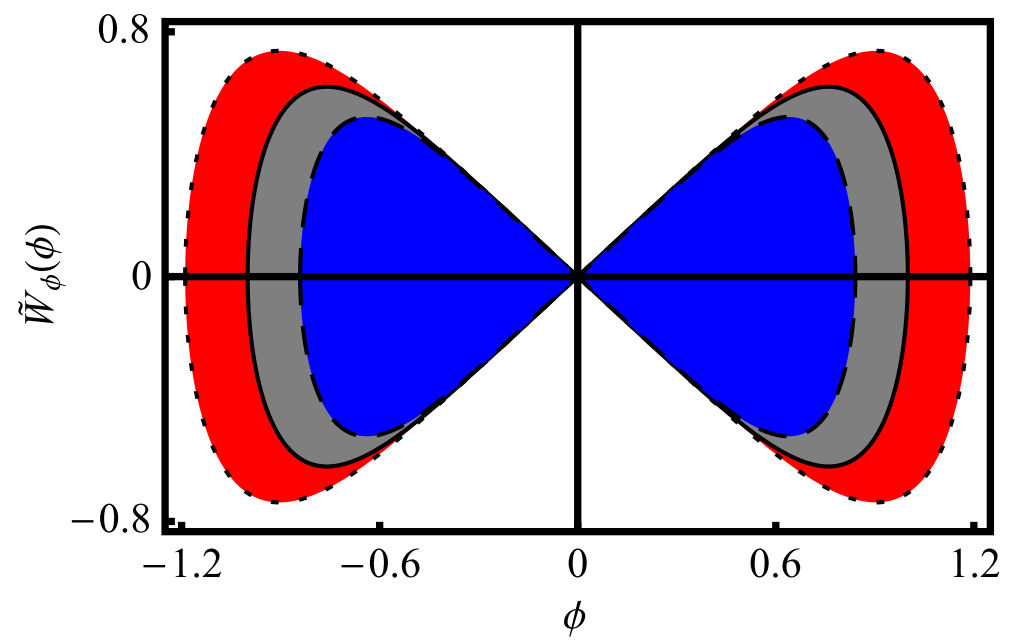

Fig. 8. (Color online) Gradient $W_{\phi}(\phi)$ of the superpotentials for $\phi>0$ and $\phi<0$. As before, the case $\chi=0.5$ corresponds to the punctuated line, $\chi=1$ to the solid line and $\chi=2$ to the dashed line. 
In 2D, one can use the light-cone coordinates

$$
\xi:=\frac{1}{2}(\tilde{x}+c \tilde{t}), \quad \eta:=\frac{1}{2}(\tilde{x}-c \tilde{t})
$$

in order to rewrite the field equation as

$$
\phi_{\xi \eta}=\frac{\partial V}{\partial \phi}=\phi\left(1-3 \chi \phi^{4}\right) \text {. }
$$

Let us now look for a relation between the first partial derivatives and operators $P$ and $Q$ as follows:

$$
\begin{aligned}
& \phi_{\xi}=P(\phi), \\
& \phi_{\eta}=Q(\phi),
\end{aligned}
$$

to which we refer to as BTs. The second derivatives are linked via the compatibility conditions

$$
\begin{aligned}
\phi_{\eta \xi} & =P_{\phi} \phi_{\eta}=P_{\phi} Q, \\
\phi_{\xi \eta} & =Q_{\phi} \phi_{\xi}=Q_{\phi} P .
\end{aligned}
$$

Then the operators $P$ and $Q$ can be obtained by simple integration as follows:

$$
P=\frac{Q}{a^{2}}= \pm \frac{1}{a} \sqrt{\phi^{2}\left(1-\chi \phi^{4}\right)+c_{2}}
$$

such that the Bäcklund transformations read explicitly

$$
\phi_{\xi}= \pm \frac{1}{a^{2}} \phi_{\eta}= \pm \frac{1}{a} \sqrt{\phi^{2}\left(1-\chi \phi^{4}\right)+c_{2}} .
$$

Now let us apply an inverse Lorentz boost

$$
\begin{aligned}
\zeta & :=\gamma(\tilde{x}+u \tilde{t})=a^{-1} \xi+a \eta=\frac{(c \tilde{t}+\tilde{x})}{2 a}+\frac{a(-c \tilde{t}+\tilde{x})}{2} \\
& =\frac{a^{-1}+a}{2}\left(\tilde{x}+\frac{1-a^{2}}{1+a^{2}} c \tilde{t}\right),
\end{aligned}
$$

where $u$ is the velocity of the soliton and

$$
\gamma:=\left(1-\frac{u^{2}}{c^{2}}\right)^{-1 / 2}=\frac{a+a^{-1}}{2}
$$

the Lorentz factor. In Eq. (40), the light-cone coordinates (33) are parameterized by the factor

$$
a= \pm \sqrt{\frac{c-u}{c+u}}, \quad u=c \frac{1-a^{2}}{1+a^{2}} .
$$

known from the relativistic Doppler shift of frequencies. In the case $c_{2}=0$, an integration of Eq. (39) leads to

$$
\pm \int \frac{d \phi}{\phi \sqrt{1-\chi \phi^{4}}}=\int \frac{1}{a} d \xi+\int a d \eta
$$

or, after inversion, to the rather explicit form

$$
\phi=\frac{1}{\sqrt[4]{\chi}} \sqrt{\operatorname{sech}(2 \zeta+b)}
$$

generalizing Eq. (28), where $b$ is another integration constant. In order to generate multi-soliton solutions, one may consider the following nonlinear superposition of (35) or (36) which, in the 
particular Lane-Emden case, involve (39). Then the BTs adopt the form

$$
\left(\phi^{(1)}+\alpha \phi^{(0)}\right)_{\xi}= \pm \frac{1}{a}\left(\phi^{(1)}-\alpha \phi^{(0)}\right) \sqrt{1-\chi\left(\phi^{(1)}-\alpha \phi^{(0)}\right)^{4}}
$$

and

$$
\left(\phi^{(1)}-\alpha \phi^{(0)}\right)_{\eta}= \pm a\left(\phi^{(1)}+\alpha \phi^{(0)}\right) \sqrt{1-\chi\left(\phi^{(1)}+\alpha \phi^{(0)}\right)^{4}},
$$

where $\alpha$ is another constant. Using the corresponding Bianchi permutability theorem $[33,30]$, we have at our disposal the completely algebraic chain

$$
\phi^{(3)}=B_{2} \phi^{(1)}=B_{1} \phi^{(2)}=B_{2} B_{1} \phi^{(0)}=B_{1} B_{2} \phi^{(0)}
$$

of transformations in order to generate the solutions $\phi^{(i)}$, with $i=0,1,2,3$. In our case we find

$$
\begin{aligned}
& \left(\phi^{(3)}-\alpha \phi^{(1)}\right) \sqrt{1-\chi\left(\phi^{(3)}-\alpha \phi^{(1)}\right)^{4}}-\alpha\left(\phi^{(2)}-\alpha \phi^{(0)}\right) \sqrt{1-\chi\left(\phi^{(2)}-\alpha \phi^{(0)}\right)^{4}} \\
& =\frac{a_{2}}{a_{1}}\left\{\left(\phi^{(3)}-\alpha \phi^{(2)}\right) \sqrt{1-\chi\left(\phi^{(3)}-\alpha \phi^{(2)}\right)^{4}}\right. \\
& \left.\quad-\alpha\left(\phi^{(1)}-\alpha \phi^{(0)}\right) \sqrt{1-\chi\left(\phi^{(1)}-\alpha \phi^{(0)}\right)^{4}}\right\} .
\end{aligned}
$$

In order to resolve this algebraic equation for $\phi^{(3)}$, we can choose the vacuum 'seed' solution $\phi^{(0)}=0$ and, additionally, are departing for $\phi^{(1)} \mathrm{y} \phi^{(2)}$ from the two solitons of the form (44). Since Eq. (48) is highly nonlinear, only a numerical resolution appears to be feasible.

\subsection{Approximate Bäcklund transformation}

However, adopting the strategies of Refs. [40-42], an approximate transformation can be obtained after noting that, in light-cone coordinates, the sine-Gordon equation (12) acquires the form

$$
\theta_{\xi \eta}=\sin \theta
$$

and has the exact solution

$$
\theta=4 \arctan [\exp \gamma(\tilde{x}-u \tilde{t})] \text {. }
$$

This can be related to the Lane-Emden solution (44) with $b=0$, via the mapping

$$
\phi=\phi(\theta)=\frac{1}{\sqrt[4]{\chi}}[\sin (\theta / 2)]^{1 / 2}=\frac{1}{\sqrt[4]{\chi}} \sqrt{\operatorname{sech}(2 \zeta)},
$$

still being exact due to the identity $\sin (2 \arctan y)=\operatorname{sech}(\ln y)$ for (hyperbolic) trigonometric functions.

Let us now turn to the multi-kink solution of the sG equation. In particular, let us consider the collision of a kink and antikink in the form

$$
\theta_{\mathrm{k} \overline{\mathrm{k}}}=4 \arctan \left(A \frac{\sinh \left(\frac{\zeta_{1}-\zeta_{2}}{2}\right)}{\cosh \left(\frac{\zeta_{1}+\zeta_{2}}{2}\right)}\right)
$$

where

$$
A=\frac{a_{1}+a_{2}}{a_{1}-a_{2}}=\frac{c^{2}-u_{1} u_{2} \pm c^{2} \gamma_{1}^{-1} \gamma_{2}^{-1}}{c\left(u_{2}-u_{1}\right)}
$$

is dimensionless, resembling the inverse of the relativistic addition theorem $\left(u_{2}-u_{1}\right) /\left(1-u_{1} u_{2} / c^{2}\right)$ for the velocity. Here the Lorentz factor (41) and the Doppler shift (42) for different velocities $u_{1}$ and $u_{2}$ are employed. 
In Fig. 9, in which the graphical representations of Ref. [43] are adopted, the absolute value of the derivative of the kinks is drawn in the case of the sine-Gordon equation. Since this is more localized, the transition between different topological sectors of the kinks becomes more transparent. Moreover, it facilitates a subsequent comparison with the scattering behavior of solitons.

Finally, let us now apply the mapping (51) to the Lane-Emden case in order to generate the two soliton solution

$$
\begin{aligned}
\phi\left(\theta_{\mathrm{k} \mathrm{k}}\right) \sqrt[4]{\chi} & =\left(\sin \left\{2 \arctan \left[A \frac{\sinh \left(\frac{\zeta_{1}-\zeta_{2}}{2}\right)}{\cosh \left(\frac{\zeta_{1}+\zeta_{2}}{2}\right)}\right]\right\}\right)^{1 / 2} \\
& =\left(\operatorname { s e c h } \left\{\operatorname { l n } \left[A \frac{\sinh \left(\frac{\zeta_{1}-\zeta_{2}}{2}\right)}{\left.\left.\left.\cosh \left(\frac{\zeta_{1}+\zeta_{2}}{2}\right)\right]\right\}\right)^{1 / 2}}\right.\right.\right. \\
& =\sqrt{\frac{2 A\left(\sinh \zeta_{1}-\sinh \zeta_{2}\right)}{A^{2}\left\{\cosh \left(\zeta_{1}-\zeta_{2}\right)-1\right\}+\left\{\cosh \left(\zeta_{1}+\zeta_{2}\right)+1\right\}}},
\end{aligned}
$$

where again identities for trigonometric (hyperbolic) functions are applied.

In the resulting Fig. 10, the scattering of two solitons behaves as expected. After crossing the collision region, the individual solitons regain their original velocities, their trajectories are asymptotically the same as the initial ones, and the phase shift appears reasonable.

However, due to some inelastic effects [41], the amplitudes of the two solitons do not vary according to their different initial velocities. This appears to be an artifact of our approximation, in as much it is not an exact solution to the nonlinear Klein-Gordon equation (34). So far the (average) deviation from the exact BT (48) has not been analyzed in detail.

\section{Outlook: soliton type collisions in galaxy clusters?}

Galaxy clusters, during their collisions million years ago, may be an essential key for understanding the evolution of the whole Universe. In fact, for Zwicky [1] the observed dynamics of galaxies in the Coma cluster provided the first clue in postulating already 1933 'missing matter'.

The so-called 'bullet cluster' (catalog number 1E0657-558) is regarded as the most convincing indirect evidence [10] for the existence of DM. During a collision or merging of galaxy clusters with velocities up to $4700 \mathrm{~km} / \mathrm{s}$, the DM halos monitored via microlensing [44] are displaced from the gas and stars and seem to pass each other in a soliton-like way. During the huge collision taking place in the bullet cluster, the hot gas (shown in red and detected by the Chandra X-ray observatory) in each cluster is slowed and distorted by a drag force, similar to air resistance. A bullet-shaped cloud of gas forms in one of the clusters. In contrast, DM (shown in blue and only observable via microlensing) does not slow down by the impact because it does not interact directly with the gas except through gravity. Therefore, the DM lumps from the two clusters move ahead of the hot gas, producing a separation of dark and visible matter, as envisioned in Fig. 11.

Although the observations in merging cluster can be given a rather conventional interpretation via high-resolution hydrodynamical simulations [45] of the baryonic and DM components, axionic BECs offer the possibility of lump-like DM structures [31], similarly to those of boson stars [22]. Since solitons are rather stable entities, they behave effectively like colliding particles, i.e. after leaving the interaction region where they may deform, they ultimately return to their original shapes and velocities. Since the DM distributions in the bullet cluster appear not to be affected during merging, one may speculate [46,47] about a solitary wave behavior of this component of galaxies and clusters, as are exhibiting our previous 2D toy models for the axion field or its Lane-Emden truncation.

More recently, a mysterious central 'dark core' [11] in the Abell cluster 520 seems to present another challenge to standard CDM. In our tentative soliton type model of DM, however, such a 'sticky' behavior of DM can be associated with that stage of merging of the two Lane-Emden lumps just before or after the scattering point, the origin of Fig. 10. In contradistinction, in the case of the bullet cluster, 

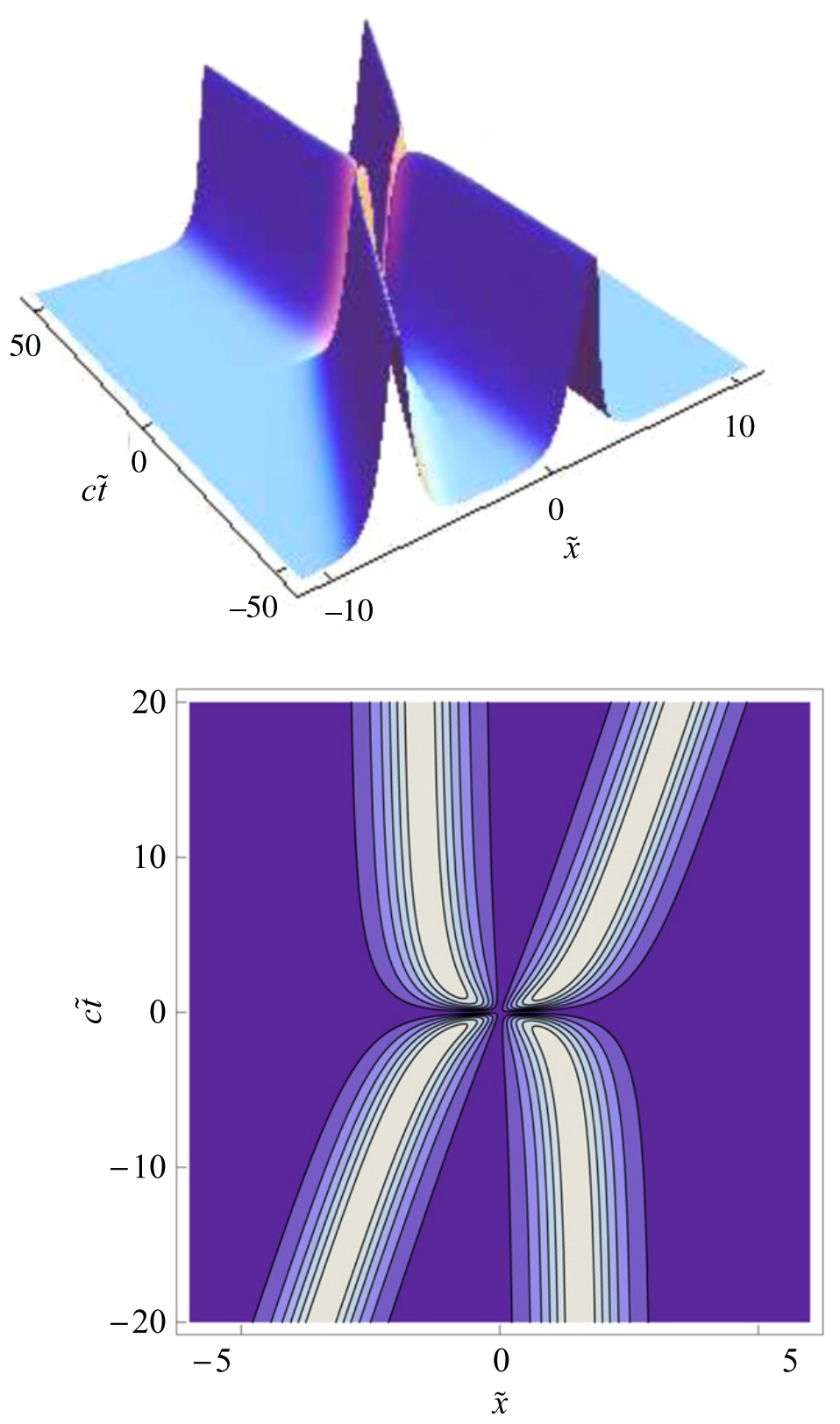

Fig. 9. (Color online) Derivative of the exact solution $\theta_{\mathrm{k} \overline{\mathrm{k}}}$ in a Minkowski type spacetime diagram. The contour plot of the collision region clearly exhibits the 'annihilation' of the kink and antikink, later on recovering their respective shapes, albeit a phase shift.

the DM lumps appear already well separated due to their rather high mutual 'infall' velocities; cf. Ref. [48]. The galaxy cluster DLSCL J0916.2+2951 is rather similar, inasmuch as the separation of dark and normal matter is concerned, except for the important difference [49] that its collision is older and slower. In our 2D model, there occurs a phase shift of the asymptotic velocities of the colliding solitons. This could be regarded as a hint for an intrinsic mechanism explaining the enhanced infall velocity of the bullet cluster, but this issue needs future confirmation via careful studies of 3D approximations.

On the cosmological scale, it has been recently realized [50] that axions not only constitute a viable DM candidate but as a coherent BEC type state [51] could provide a mechanism in order to reconcile the observed and theoretically predicted value of the primordial abundance of ${ }^{7} \mathrm{Li}$. As mentioned before, the Lane-Emden truncation provides only meta-stable lumps, whose decay time $\tau \simeq \lambda$ compt $/ c$ is proportional to the Compton wave length [4] of the very light particle. Since this is shorter than the 

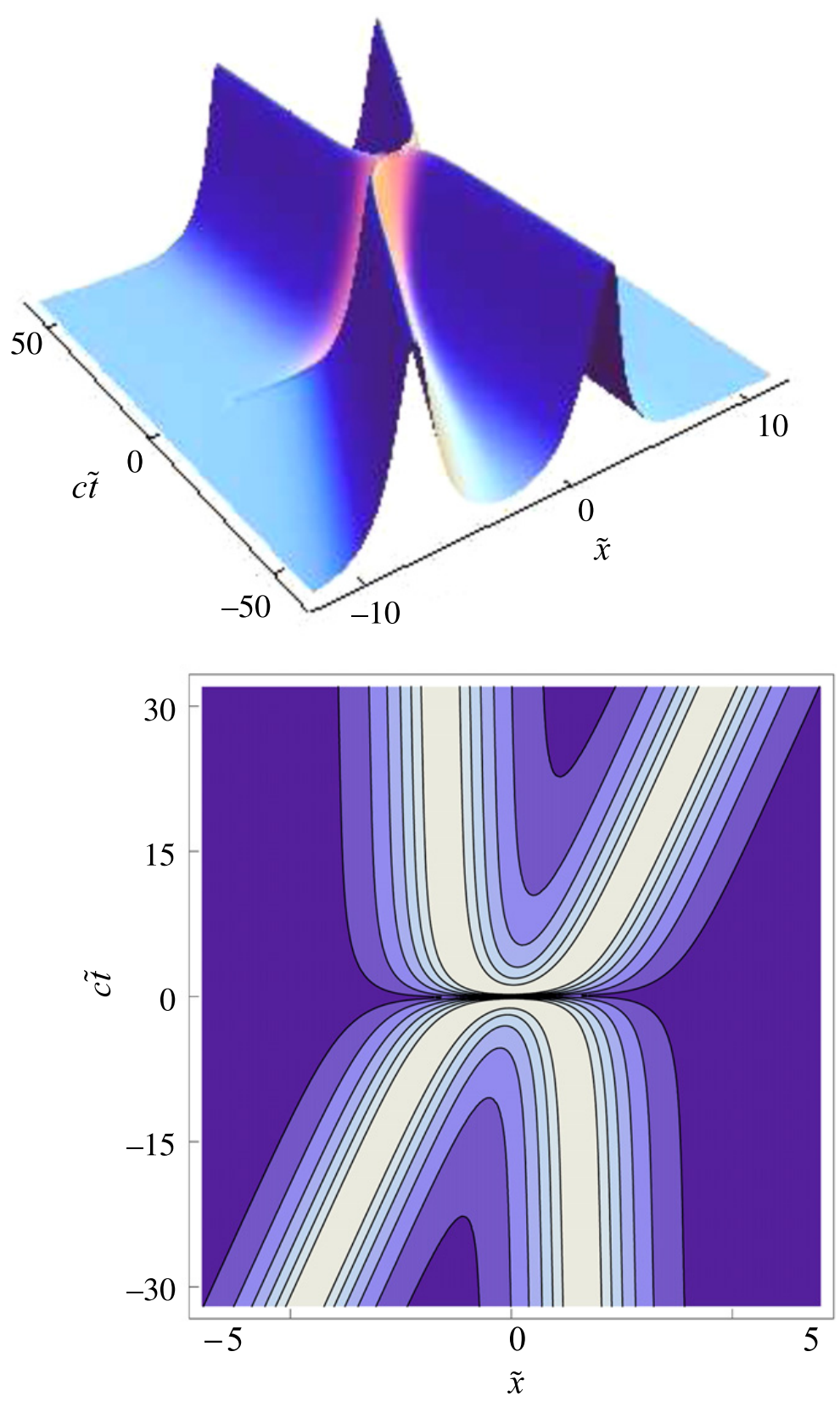

Fig. 10. (Color online) Approximation of a collision of two solitons in the Lane-Emden $\phi^{6}$ model. The contour plot amplifies the scattering region of our spacetime diagram.

collision time as well as the age of the Universe, one needs to consider the stabilizing 'trap' generated by self-gravity, similarly as in the absolutely stable branches [52] of boson stars. Then the very study of clusters could provide constraints on DM parameters such as their lifetime [53].

Thus it appears prospective to generalize the scattering of two Lane-Emden solitons in 2D to more realistic 3D (head-on) collisions of self-gravitating lumps [5,46,54], similar to those depicted in Fig. 12, albeit formidable requirements on the computational resources.

\section{Acknowledgments}

The constructive report of the referee and the support of the SNI is gratefully acknowledged. One of us (DCV) was supported by CONACyT, grant 211353, whereas EWM thanks Noelia Méndez Córdova, 


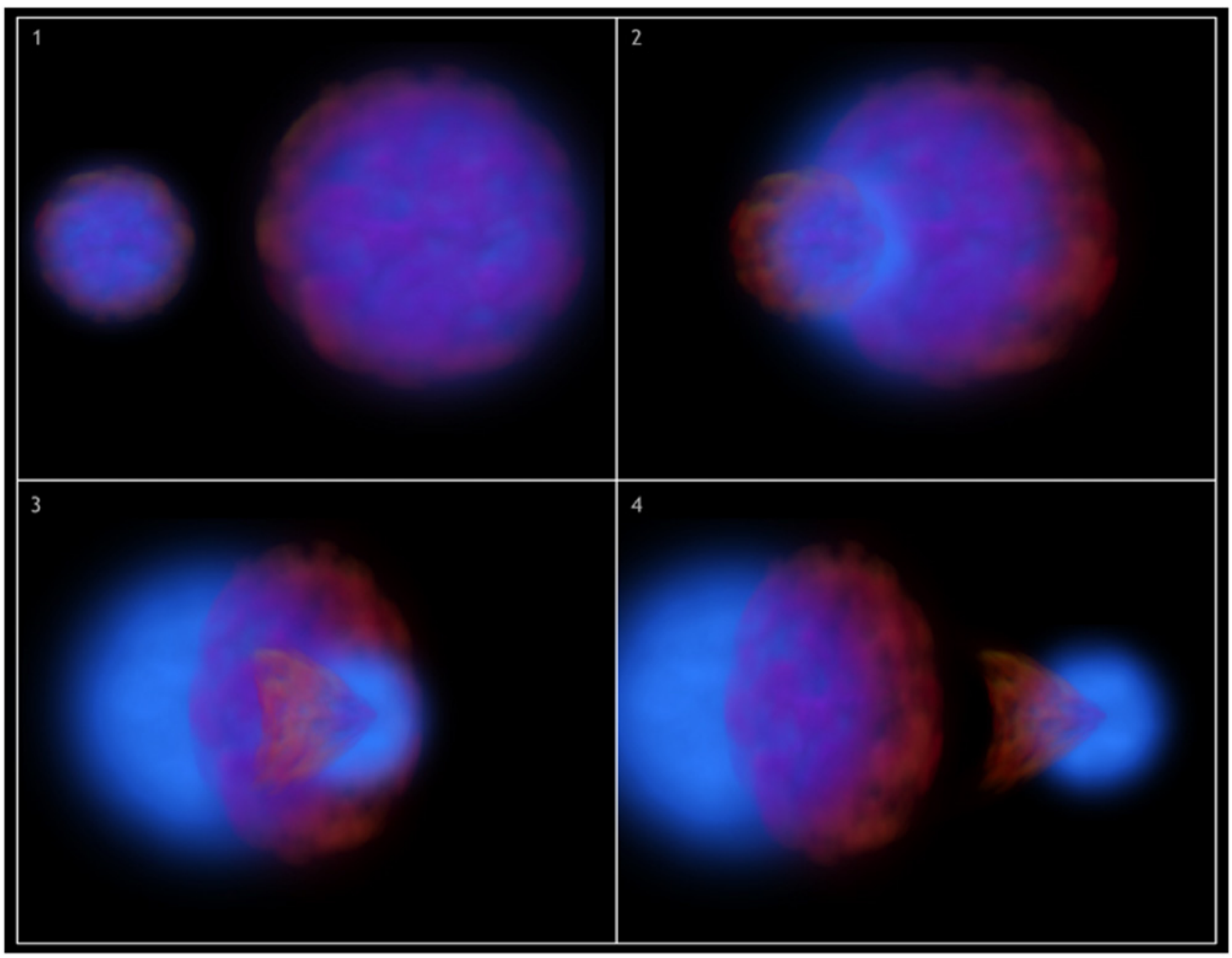

Fig. 11. (Color online) Four stages of the collision presumably taking place in the bullet cluster, according to an artist's view. (Credit: NASA/CXC/M. Weiss.)

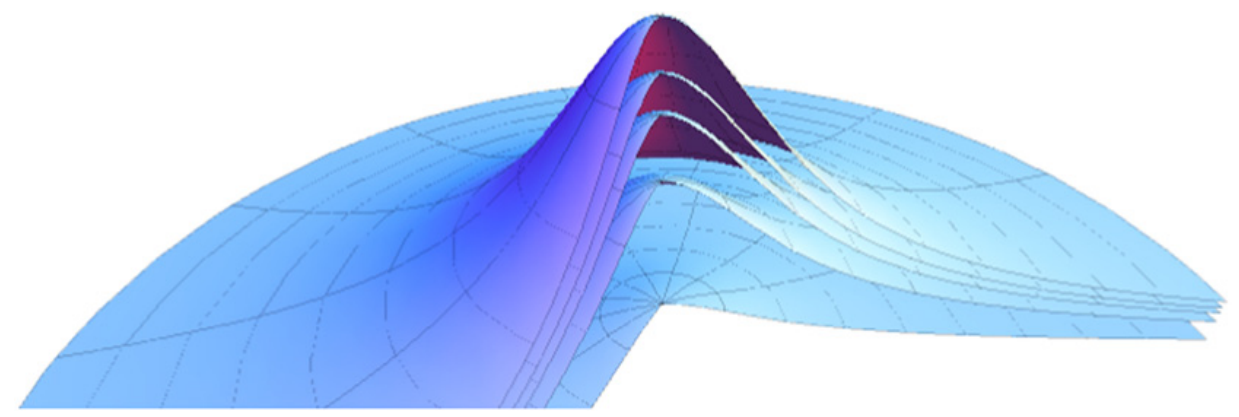

Fig. 12. (Color online) Radial profile of exact lump type solutions of the Lane-Emden equation in 3D.

Miryam Sophie Naomi, and Markus Gérard Erik for encouragement. The work is dedicated to Harald Mielke.

\section{References}

[1] F. Zwicky, Helv. Phys. Acta 6 (1933) 110.

[2] Johanna Miller, Phys. Today 12 (2012).

[3] S.-J. Sin, Phys. Rev. D 50 (1994) 3650.

[4] E.W. Mielke, Phys. Rev. D 18 (1978) 4525-4528.

[5] E.W. Mielke, F.E. Schunck, Phys. Rev. D 66 (2002) 023503.

[6] B. Fuchs, E.W. Mielke, Mon. Not. R. Astron. Soc. 350 (2004) 707-709.

[7] E.W. Mielke, J.A. Vélez Pérez, Phys. Rev. D 75 (2007) 043504.

[8] T. Rindler-Daller, P.R. Shapiro, Mon. Not. R. Astron. Soc. 422 (2012) 135-161. 
[9] E.W. Mielke, J.A. Vélez Pérez, Phys. Lett. B 671 (2009) 174-178.

[10] D. Clowe, M. Bradac, A.H. Gonzalez, M. Markevitch, S.W. Randall, C. Jones, D. Zaritsky, Astrophys. J. 648 (2006) L109.

[11] M.J. Jee, A. Mahdavi, H. Hoekstra, A. Babul, J.J. Dalcanton, P. Carroll, P. Capak, Astrophys. J. 747 (2012) 96.

[12] N.J. Zabusky, M.D. Kruskal, Phys. Rev. Lett. 15 (1965) 240-243.

[13] R.K. Dodd, J.C. Eilbeck, J.D. Gibbon, H.C. Morris, Solitons and Nonlinear Wave Equations, Academic Press, London, 1982.

[14] R. Rajaraman, Solitons and Instantons, North-Holland, Amsterdam, 1982.

[15] A. Barone, F. Esposito, C. Magee, A. Scott, Nuovo Cimento 1 (1971) 227.

[16] F.V. Kusmartsev, Phys. Scr. 29 (1984) 7.

[17] P. Kumar, B.S. Thomas, Phys. Rev. B 37 (1988) 683.

[18] A.T. Avelar, D. Bazeia, W.B. Cardoso, Nonlinear Anal. RWA 11 (2010) 4269.

[19] D.R. Gulevich, F.V. Kusmartsev, S. Savel'ev, V.A. Yampol'skii, F. Nori, Phys. Rev. Lett. 101 (2008) 127002.

[20] A.G. Lisi, J. Phys. A: Math. Gen. 28 (1995) 5385.

[21] R. Teisseyre, M. Takeo, E. Majewski (Eds.), Earthquake Source Asymmetry, Structural Media and Rotation Effects, Springer, New York, 2006.

[22] E.W. Mielke, F.E. Schunck, Nuclear Phys. B 564 (2000) 185.

[23] J. Ruostekoski, Phys. Rev. A 70 (2004) 041601.

[24] E.W. Mielke, B. Fuchs, F.E. Schunck, in: M. Novello, S. Perez-Bergliaffa, R. Ruffini (Eds.), Proc. of the Tenth Marcel Grossman Meeting on General Relativity, Rio de Janeiro, 2003, World Scientific, Singapore, 2006.

[25] J.E. Kim, G. Carosi, Rev. Modern Phys. 82 (2010) 557.

[26] Y. Brihaye, T. Delsate, Phys. Rev. D 78 (2008) 025014.

[27] P. Sikivie, Q. Yang, Phys. Rev. Lett. 103 (2009) 111301.

[28] J. Mielczarek, T. Stachowiak, M. Szydlowski, Int. J. Mod. Phys. D 19 (2010) 1843-1855.

[29] E.B. Bogomol'nyi, Sov. J. Nucl. Phys. 24 (1976) 449.

[30] N. Manton, P. Sutcliffe, Topological Solitons, Cambridge University Press, Cambridge, 2004.

[31] A.T. Avelar, D. Bazeia, L. Losano, R. Menezes, Eur. Phys. J. C55 (2008) 133-143.

[32] A.T. Avelar, D. Bazeia, W.B. Cardoso, L. Losano, Phys. Lett. A 374 (2009) 222-227.

[33] C. Rogers, W.K. Schief, Bäcklund and Darboux Transformations: Geometry and Modern Applications in Soliton Theory, Cambridge University Press, Cambridge, 2002.

[34] F.K. Liu, Mon. Not. R. Astron. Soc. 281 (1996) 1197.

[35] K. Parand, M. Dehghan, A.R. Rezaei, S.M. Ghaderi, Comput. Phys. Comm. 181 (2010) 1096-1108.

[36] K. Parand, A.R. Rezaei, A. Taghavi, Acta Astronaut. 67 (2010) 673.

[37] L. Vazquez, J. Math. Phys. 18 (1977) 1341.

[38] P. Dorey, K. Mersh, T. Romanczukiewicz, Y. Shnir, Phys. Rev. Lett. 107 (2011) 091602.

[39] C.S. Gardner, J.M. Green, M.D. Kruskal, R.M. Miura, Phys. Rev. Lett. 19 (1967) 1095-1097.

[40] R.F. Dashen, B. Hasslacher, A. Neveu, Phys. Rev. D 11 (1975) 3424.

[41] V.G. Makhankov, Phys. Rep. 35 (1978) 1.

[42] M. Jia, F. Huang, S.Y. Lou, Phys. Scr. 73 (2006) 368.

[43] K. Brauer, The Korteweg-de Vries equation: history, exact solutions, and graphical representation, University of Osnabrück, May 2006.

[44] F.E. Schunck, B. Fuchs, E.W. Mielke, Mon. Not. R. Astron. Soc. 369 (2006) 485.

[45] C. Mastropietro, A. Burkert, Mon. Not. R. Astron. Soc. 389 (2008) 967-988.

[46] A. Bernal, F. Siddhartha Guzman, Phys. Rev. D 74 (2006) 103002.

[47] J.W. Lee, S. Lim, D. Choi, arXiv:0805.3827 [hep-ph].

[48] J. Lee, M. Baldi, Astrophys. J. 747 (2012) 45.

[49] W.A. Dawson, et al., Astrophys. J. Lett. 747 (2012) L42.

[50] O. Erken, P. Sikivie, H. Tam, Q. Yang, Phys. Rev. Lett. 108 (2012) 061304

[51] E.W. Mielke, F.E. Schunck, Gen. Relativity Gravitation 33 (2001) 805-813.

[52] F.V. Kusmartsev, E.W. Mielke, F.E. Schunck, Phys. Rev. D 43 (1991) 3895.

[53] S. Riemer-Sorensen, K. Zioutas, S.H. Hansen, K. Pedersen, H. Dahle, A. Liolios, Phys. Rev. Lett. 99 (2007) 131301.

[54] D.-I. Choi, Phys. Rev. A 66 (2002) 063609; D. Choi, C.W. Lai, M.W. Choptuik, E.W. Hirschmann, S.L. Liebling, F. Pretorius, Dynamics of axisymmetric (head-on) miniboson star collisions, 2010, Preprint. 


\title{
Increased infall velocities in galaxy clusters from solitonic collisions?
}

\author{
David Castañeda Valle ${ }^{*}$ and Eckehard W. Mielke ${ }^{\dagger}$ \\ Departamento de Física, Universidad Autónoma Metropolitana Iztapalapa, Apartado Postal 55-534, \\ C.P. 09340, México, D.F., Mexico \\ (Received 14 October 2013; published 12 February 2014)
}

\begin{abstract}
Axionlike scalar fields and the Lane-Emden truncation of their periodic potential are analyzed as a model of dark matter halos. The apparent enhancement of infall velocities in merging clusters is intriguing: here it is tentatively explained via an intrinsic inelastic effect during relativistic soliton collisions.
\end{abstract}

DOI: 10.1103/PhysRevD.89.043504

\section{INTRODUCTION}

Head-on collisions of massive galaxy clusters, like those occurring in the so-called bullet cluster [1] as well as the gigantic merger Abell 520 possibly containing a dark matter (DM) core [2], are a challenge for the cold dark matter (CDM) paradigm, because both the individual galaxies and the hot $\mathrm{x}$-ray emitting interstellar gas are only partially anchored to the DM lumps.

Instead of SUSY-type WIMPs, we are probing axionlike particles [3-5] as dark matter candidates. Observations of astrophysical collisions such as merging clusters provide a valuable test of alternatives to CDM. In particular, we continue our investigation [6] whether or not two dark matter halos, pulling towards one another, can be modeled via soliton-type collisions without invoking [7] a dark energy (DE) mediating "fifth force." The (phase) displacement of two colliding solitons, to some extent, resembles the effects of such a hypothetical force not observed in the laboratory [8]. In cosmology, however, there are arguments [9] to ponder about a quintessencelike scalar field playing the role of DE.

Since solitons are rather stable entities, they behave effectively like colliding particles; i.e., after leaving the interaction region where they may deform due to a temporally inelastic mechanism studied here in a relativistic setting, they ultimately return to their original shapes and velocities. Since the DM distributions in the bullet cluster appear not to be affected during merging, we postulate an axionlike scalar component of galaxies and clusters and analyze its solitary wave behavior, or as in our previous two-dimensional toy model [6], its Lane-Emden (LE) truncation [10],

$$
V_{\mathrm{LE}}(\phi)=\frac{m^{2}}{2} \phi^{2}\left(1-\chi \phi^{4}\right) .
$$

Recall that in quantum chromodynamics (QCD) axions of inertial mass $m$ are self-interacting via the effective $[11,12]$ periodic potential,

\footnotetext{
casvada@gmail.com

†ekke@xanum.uam.mx
}

PACS numbers: 95.35.+d, 03.75.Lm, 14.80.Va, 98.65.Cw

$$
\begin{aligned}
V(\phi) & =\frac{m^{4}}{\lambda}\left[1-\cos \left(\frac{\sqrt{\lambda}}{m} \phi\right)\right] \\
& \simeq V_{\mathrm{LE}}(\phi)-\frac{\lambda}{4 !} \phi^{4}-\cdots
\end{aligned}
$$

Although globally unbounded due to $\chi=-\lambda^{2} /\left(360 m^{4}\right)$, the LE truncation (1) has the advantage that, in three dimensions, it admits exact spherically symmetric solutions [10] which model quite well [13] DM halos of individual galaxies. More specifically, the LE equation provides us, in three dimensions, with the exact nonsingular [14] radial solution,

$$
|\phi(r)|=\chi^{-1 / 4} \sqrt{\frac{A}{1+A^{2} r^{2}}} .
$$

This is referred to as a metastable lump, cf. Fig. 12 of Ref. [6], which cannot easily be confounded with domain walls. More than a century ago, it was considered as a crude model for the density of the sun and a bit later for the distribution of stars in globular clusters.

Our previous proposal $[15,16]$ that DM may be composed of a gas of "axion mini clusters" or mini axion stars has been recently adopted [17]. However, numerical simulations of pointlike objects would again run into a "cusp" in the density of the central core, which is in conflict with observations of low-surface brightness galaxies. Moreover, for such BoseEinstein type condensates, one needs some self-interaction and, in view of the self-similarity of solitons, one would end up with much larger configurations resembling the lumptype halos we will consider here.

\section{RELATIVISTIC KINEMATICS OF INELASTIC COLLISIONS}

As is well known, the wave operator $\square:=\nabla \cdot \nabla-$ $\partial^{2} / c^{2} \partial t^{2}$ is invariant under the standard Lorentz transformations [18], where $c$ is the velocity of light in vacuum. Thus one can resolve the relativistically invariant semiclassical Klein-Gordon (KG) equation, 


$$
\square \phi=\frac{\partial V(\phi)}{\partial \phi},
$$

for a single scalar field $\phi=\phi(t, \vec{x})$ first in some rest frame. Then, we apply a Lorentz boost

$$
\vec{x} \rightarrow \gamma(\vec{x}-\vec{u} t),
$$

in order to get traveling wave solutions or "moving solitons" needed for studying their collisons. Here,

$$
\gamma:=1 / \sqrt{1-\vec{\beta} \cdot \vec{\beta}}=\frac{D+D^{-1}}{2}
$$

is the Lorentz factor, $\vec{u}$ the (phase) velocity of the solitary wave, $\vec{\beta}=\vec{u} / c$ the dimensionless relative velocity, and

$$
D:=\sqrt{\frac{c+u}{c-u}}, \quad u=c \frac{D^{2}-1}{D^{2}+1},
$$

the relativistic Doppler shift. [19]

An inverse Lorentz boost is later denoted by

$$
\begin{aligned}
\zeta & :=\gamma(\tilde{x}+u \tilde{t}) \\
& =\frac{D^{-1}+D}{2}\left(\tilde{x}-\frac{1-D^{2}}{1+D^{2}} c \tilde{t}\right) \\
& =\xi D+\eta D^{-1} .
\end{aligned}
$$

In the last equation we have made use of the fact that, in two dimensions, solutions of the KG equation can conveniently be written in terms of light-cone coordinates

$$
\xi:=\frac{1}{2}(\tilde{x}+c \tilde{t}), \quad \eta:=\frac{1}{2}(\tilde{x}-c \tilde{t}) .
$$

After dividing by the Compton wave length $\lambda_{\text {Compton }}=$ $h / m c$ of a particle, i.e., $\tilde{x}:=x / \lambda_{\text {Compton }}$ and $\tilde{t}:=t / \lambda_{\text {Compton }}$, these coordinates become dimensionless.

Let us now consider inelastic collisions of two particles of mass $m_{1}$ and $m_{2}$, respectively, moving with velocities $\vec{u}_{1}$ and $\vec{u}_{2}$, as measured from a positive oriented frame. From the conservation of the relativistic energy $E=\gamma m c^{2}$ and relativistic momenta $\vec{p}=\gamma m \vec{u}$ in two dimensions, there results [20] the collinear velocity

$$
U=\frac{m_{1} \gamma_{1} u_{1}+m_{2} \gamma_{2} u_{2}}{m_{1} \gamma_{1}+m_{2} \gamma_{2}}
$$

of the (newly generated) composite particle of mass

$$
M=\frac{m_{1} \gamma_{1}+m_{2} \gamma_{2}}{\gamma(U)}
$$

Here the Lorentz factor (6) applies for different velocities $u_{1}, u_{2}$, and $U$.
For particles of initially equal masses $m_{1}=m_{2}$, Eq. (10) degenerates to

$$
\mathbb{B}:=\frac{U}{c}=\frac{\gamma_{1} \beta_{1}+\gamma_{2} \beta_{2}}{\gamma_{1}+\gamma_{2}} \equiv \frac{1+\beta_{1} \beta_{2}-\gamma_{1}^{-1} \gamma_{2}^{-1}}{\beta_{1}+\beta_{2}} .
$$

This dimensionless common velocity, coinciding with Eq. (3.11) of Ref. [21], also arises in the multisoliton solutions. At times, $u_{1}=-\left|\vec{u}_{1}\right|$ is adopted in the center of the collision frame. Thus, we can surmise that in some soliton collisions an intermediate state [22] occurs which is temporarily inelastic. Indirectly, this may affect all nonlinear waves [23] due to Doppler broadening of the shape of interacting solitons.

Quite generally, not only inelastic collisions of point masses but also the peaks of multisolitons are suffering from some "sticky" or inelastic interaction during merging. A long way after the collision, however, the only remnant from this intermediate state is a (phase-) shift,

$$
\delta_{i}:=\gamma_{i}^{-1} \ln |\mathbb{B}|,
$$

of the centers of the asymptotic solitons. This leads to an "overtaking process" as has been noted before [21,24,25], without revealing, to our knowledge, its elementary relativistic origin.

For light pulses, there occurs a repulsion phenomenon by which the intensity in the central collision region is decreased during the overlap of the two solitons: this leads to a decrease of the effective refractive index and, hence, an ejection of the peaks from the central region. Again, a related phase shift is observed after the collision, cf., Ref. [26].

Here we will analyze this intriguing effect in the case of the Lane-Emden truncation.

\section{SOLITON COLLISIONS}

When several solitons collide, one expects that they recuperate their initial shapes and velocities after some time has passed. Remnants from crossing the scattering region may be the concomitant phase shifts or displacements of the centers of the individual solitary waves due the nonlinear, partially inelastic interaction.

For constructing multisolitons, the well-established Bäcklund transformation (BT), cf., Refs. [25,27,28], is employed, which may also bridge between different types of nonlinear equations.

Let us depart from the sine-Gordon (sG) equation [29] which in dimensionless light-cone coordinates (9) acquires the form

$$
\theta_{\xi \eta}=\sin \theta .
$$

In a moving frame, it has the exact kink solution, 


$$
\theta=4 C \arctan [\exp \gamma(\tilde{x}-u \tilde{t})],
$$

for $C=1$ and antikink for $C=-1$. Since its spatial derivative,

$$
\theta_{\tilde{x}}=2 \gamma C \operatorname{sech}[\gamma(\tilde{x}-u \tilde{t})],
$$

becomes localized and square integrable, its absolute value will facilitate a subsequent comparison with the scattering behavior of solitons or lumps regarded as Bose-Einstein condensates $[30,31]$ of DM.

Due to Bianchi's permutability theorem of BTs, there results the "nonlinear superposition" principle

$$
\tan \left[\left(\theta_{3}-\theta_{0}\right) / 4\right]=\mathbb{B} \tan \left[\left(\theta_{1}-\theta_{2}\right) / 4\right],
$$

which allows us to construct algebraically multikink solutions of the sG equation a la Perring and Skyrme [32,33].

Our relativistic KG equation is not only Lorentz but also $C P T$ invariant, where $C: \theta \rightarrow-\theta$ is the topological charge conjugation for a real scalar and $P: \vec{x} \rightarrow-\vec{x}$ and $T: t \rightarrow-t$ are space and time reflections, respectively. This will allow us to distinguish solitons from antisolitons.

Here we focus on the collision of two kinks (instead of a collision of a kink and its CP-odd antikink, as in Ref. [6]) and obtain from the trivial seed solution $\theta_{0}=0$ the exact solution,

$$
\theta_{\mathrm{kk}}=4 \arctan \left[K\left(\zeta_{1}, \zeta_{2}\right)\right],
$$

where the kinetic factor

$$
\begin{aligned}
K\left(\zeta_{1}, \zeta_{2}\right): & =\mathbb{B} \frac{\exp \left(\zeta_{1}\right)+C \exp \left(\zeta_{2}\right)}{\exp \left(\zeta_{1}+\zeta_{2}\right)-C} \\
& =\frac{\exp \left(\zeta_{1}+\gamma_{1} \delta_{1}\right)+C \exp \left(\zeta_{2}+\gamma_{2} \delta_{2}\right)}{\exp \left(\zeta_{1}+\zeta_{2}\right)-C} \\
& \simeq \exp \left(\zeta_{1}+\gamma_{1} \delta_{1}\right)+C \exp \left(\zeta_{2}+\gamma_{2} \delta_{2}\right)
\end{aligned}
$$

depends on the initial velocities. This is also known as Hirota's formula [34].

At large separations from the interaction region, cf. Fig. 1, the solution (18) clearly decouples asymptotically into a (noninteracting) kink-kink or kink-antikink pair [24] distinguished by the sign $C= \pm 1$ of the topological charge.

\section{A. Approximate Bäcklund transformation via mapping}

For the Lane-Emden equation of interest here, an exact auto-Bäcklund transformation has not yet been found. Instead a generalized transformation will serve as a guide in constructing a multilump solution. Consider the LaneEmden potential (1) as a truncation of (2), then the corresponding nonlinear $\mathrm{KG}$ equation in light-cone coordinates simplifies to

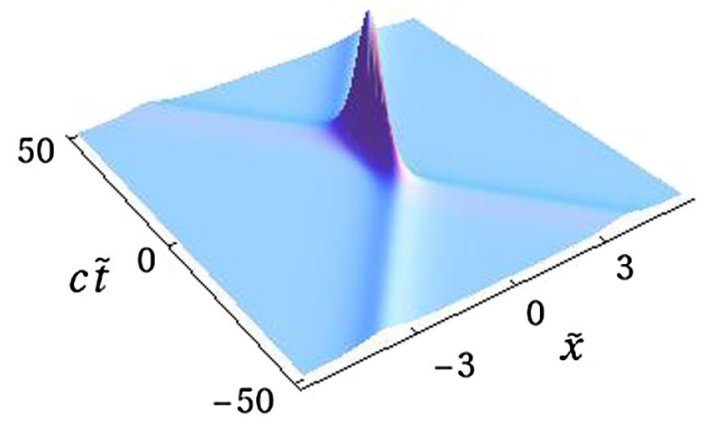

FIG. 1. (color online). Kink-kink collision monitored via the absolute value of its spatial derivative.

$$
\phi_{\xi \eta}=\frac{\partial V}{\partial \phi}=\phi\left(1-3 \chi \phi^{4}\right) .
$$

Integration leads us to the explicit exact solution

$$
\phi=\frac{1}{\sqrt[4]{\chi}} \sqrt{\operatorname{sech}(2 \zeta)}
$$

where an integration constant has been suppressed.

Due to the identity $\sin (2 \arctan y)=\operatorname{sech}(\ln y)$ for (hyperbolic) trigonometric functions, the Lane-Emden solution (21) is related to the kink (15) of the sG equation via the nonlinear mapping,

$$
\varphi=\varphi(\theta)=\frac{1}{\sqrt[4]{\chi}}[\sin (\theta / 2)]^{1 / 2}=\frac{1}{\sqrt[4]{\chi}} \sqrt{\operatorname{sech}(2 \zeta)} .
$$

This suggests that we generate a two-soliton solution,

$$
\begin{aligned}
\phi\left(\theta_{\mathrm{kk}}\right) \sqrt[4]{\chi} & =\left(\sin \left\{2 \arctan \left[K\left(\zeta_{1}, \zeta_{2}\right)\right]\right\}\right)^{1 / 2} \\
& =\left(\operatorname{sech}\left\{\ln \left[K\left(\zeta_{1}, \zeta_{2}\right)\right]\right\}\right)^{1 / 2},
\end{aligned}
$$

of the LE equation via the same mapping, using again identities for trigonometric (hyperbolic) functions. Thus far, a more precise approximation is not yet available.

In the resulting spacetime diagram, the scattering of two solitons behaves as expected: after crossing the collision region, the individual solitons regain their original velocities, their trajectories are asymptotically the same as the initial ones, and the phase shift or displacement of the centers of the lumps is completely determined by Eq. (13). For solitons, some temporary "bouncing" of the center of a lump in the collision region occurs, cf. Fig. 2, whereas an antisoliton may "tunnel" through a solition during merging, cf. Ref. [6].

Thus interacting solitons behave more like "extended particles," where due to the inelastic effects discussed above, a continuous interchange of inertia (invariant mass) and interaction energy occurs. In Ref. [24], this non-Newtonian behavior is referred to as a local version of Mach's principle, inasmuch as the total inertia of a lump depends also on the relative motion of other solitons in its vicinity. 
Although Eq. (23) is not an exact solution to the nonlinear KG equation (20), the same relativistic factor occurs in the LE truncation.

\section{DISCUSSION}

In astrophysical scenarios, the Compton wave length of DM particles is of the order of the size of a galaxy, i.e., $\lambda_{\text {Compton }}=h / m c \sim 10 \mathrm{kpc}$, but above the Hubble scale of $10^{-31} \mathrm{eV}$. This naive estimate would lead to an ultralight mass of $m \simeq 10^{-26} \mathrm{eV}$, which is 20 orders of magnitude below the usual mass range $m_{a} \simeq \mu \mathrm{eV}$ of invisible axions. Another possibility is that, in a first stage, $\mu \mathrm{eV}$ axions collapse to mini-machos [16], which then form the halo via large DM clouds.

A mysterious central "dark core" [2] in the merging cluster Abell 520 presents another challenge to standard CDM (cf., however, Ref. [35]). In our tentative soliton-type model of DM, however, such a "sticky" behavior of DM can be associated with the temporarily inelastic scattering of two or more LE lumps close to the origin of Fig. 2. On the contrary, in the case of the bullet cluster, the DM lumps appear already well separated due to their higher mutual "infall" velocities, cf. Ref. [36].

The estimated collision velocities for the Bullet cluster and Abell 520 are 4700 km/s and 1066 km/s, respectively. The Musket ball clusters appear much older [37] and slower.

\begin{tabular}{lcc}
\hline Cluster & Velocity $U / c$ & Shift $2 \delta$ \\
\hline Bullet & 0.016 & -8.3 \\
Abell 520 & 0.0033 & -11.4 \\
\hline \hline
\end{tabular}

Thus, the intrinsic mechanism $[6,25]$ of temporarily "sticky" solitons during the non-Newtonian scattering process could, to some extent, explain the enhanced infall velocity of the bullet cluster, taking into consideration that the dimensionless displacement $2 \delta$ has to be multiplied by the Compton wave length $\lambda_{\text {Compton }}$ of axionlike particles.

In the case of the galaxy cluster CLO024 + 17, one suspects that it collided with another cluster about a billion years ago, leaving a well-separated DM ring [38] resembling the angular momentum toruses [31,39] found in the three-dimensional LE equation.

As is well known [40], the LE truncation provides only metastable lumps, whose decay time $\tau \simeq \lambda_{\text {Compton }} / c$ is
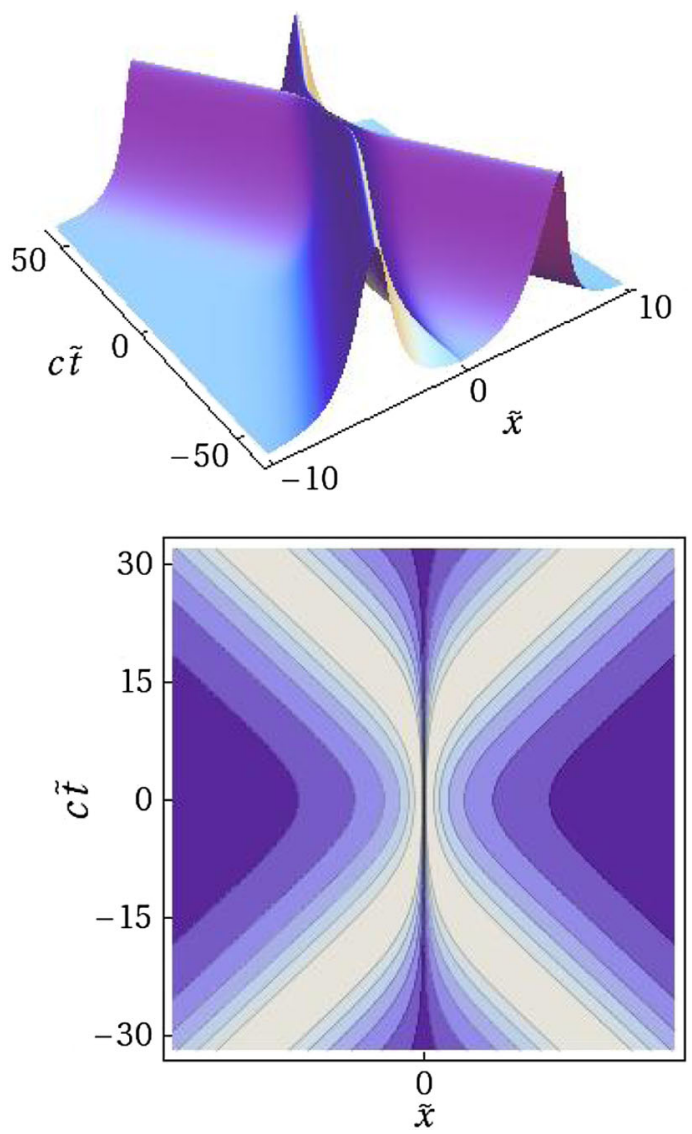

FIG. 2. (color online). Collision of two lump-type solitons in the Lane-Emden $\phi^{6}$ model. The contour plot below amplifies the scattering region of our spacetime diagram.

proportional to the Compton wave length. Since this is shorter than the collision time as well as the age of the Universe, such lumps need to be stabilized via their selfgenerated gravity [41-43], similarly as in the case of (colliding) boson stars.

\section{ACKNOWLEDGMENTS}

The support of the SNI is gratefully acknowledged. One of us (D. C. V.) was supported by CONACyT, Grant No. 211353, whereas E. W. M. thanks Noelia Méndez Córdova, Miryam Sophie Naomi, and Markus Gérard Erik for encouragement.
[1] D. Clowe, M. Bradac, A. H. Gonzalez, M. Markevitch, S. W. Randall, C. Jones, and D. Zaritsky, Astrophys. J. 648, L109 (2006).

[2] M. J. Jee, A. Mahdavi, H. Hoekstra, A. Babul, J. J. Dalcanton, P. Carroll, and P. Capak, Astrophys. J. 747, 96 (2012).
[3] P. Sikivie and Q. Yang, Phys. Rev. Lett. 103, 111301 (2009).

[4] P. Sikivie, Phys. Lett. B 695, 22 (2011).

[5] P. Sikivie, arXiv:1210.0040.

[6] D. Castañeda Valle and E. W. Mielke, Ann. Phys. (Amsterdam) 336, 245 (2013). 
[7] M. Baldi, Phys. Dark Univ. 1, 162 (2012).

[8] T. A. Wagner, S. Schlamminger, J.H. Gundlach, and E. G. Adelberger, Classical Quantum Gravity 29, 184002 (2012).

[9] M. Baldi, Ann. Phys. (Special Issue: Dark Matter) 524, 602 (2012).

[10] E. W. Mielke and F. E. Schunck, Phys. Rev. D 66, 023503 (2002).

[11] J. E. Kim and G. Carosi, Rev. Mod. Phys. 82, 557 (2010).

[12] A. Ringwald, Phys. Dark Univ. 1, 116 (2012).

[13] E. W. Mielke and J. A. Vélez Pérez, Phys. Lett. B 671, 174 (2009).

[14] P. Mach, J. Math. Phys. (N.Y.) 53, 062503 (2012).

[15] E. W. Mielke and F. E. Schunck, Nucl. Phys. B564, 185 (2000).

[16] E. W. Mielke and F. E. Schunck, Gen. Relativ. Gravit. 33, 805 (2001).

[17] J. Barranco, A. C. Monteverde, and D. Delepine, Phys. Rev. D 87, 103011 (2013).

[18] The complete Lorentz group [44] consist of the connected component $\Lambda_{\circ}$ of the identity transformation and the cosets $P \Lambda_{\circ}, T \Lambda_{\circ}$, as well as $P T \Lambda_{\circ}$, which involve the discrete group elements of space and time reflections $P$ and $T$, respectively.

[19] In the Fourier transform of hyperbolic wave equations, the Lorentz transformation $\omega \rightarrow \gamma \omega(1-\beta)=\omega / D$ of frequencies [44] is inversely proportional to the Doppler factor (7).

[20] R. D'Inverno, in Introducing Einstein's Relativity (Claredon Press, Oxford, 1995), p. 51.

[21] G. Bowtell and A. E. G. Stuart, Phys. Rev. D 15, 3580 (1977).

[22] In Ref. [45], the usual relativistic addition theorem $\left(u_{1}+u_{2}\right) /\left(1+u_{1} u_{2} / c^{2}\right)$ for the velocity has been adopted, which, however, does not account for inelastic effects.

[23] When measuring solitons in two different inertial frames, their relative normalized Doppler shift is $1 / \mathbb{B}:=\frac{D_{2} \omega-D_{1} \omega}{D_{1} \omega+D_{2} \omega}=$ $\frac{D_{2}-D_{1}}{D_{1}+D_{2}}=\left(\beta_{2}-\beta_{1}\right) /\left(1-\beta_{1} \beta_{2}-\gamma_{1}^{-1} \gamma_{2}^{-1}\right)$.

[24] I. Christov and C. I. Christov, Phys. Lett. A 372, 841 (2008).

[25] T. Taniuti and K. Nishihara, Nonlinear Waves, Monographs and Studies in Mathematics (Pitman Advanced Publishing Program, London, 1983), Vol. 15.

[26] L. Li and G. Huang, Phys. Rev. A 82, 023809 (2010).
[27] V. G. Ivancevic and T. T. Ivancevic, J. Geom. Symm. Phys. 31, 1 (2013).

[28] C. Rogers and W. K. Schief, Bäcklund and Darboux Transformations: Geometry and Modern Applications in Soliton Theory (Cambridge University Press, Cambridge, England, 2002).

[29] J. Rubinstein, J. Math. Phys. (N.Y.) 11, 258 (1970).

[30] E. W. Mielke, B. Fuchs, and F. E. Schunck, in Proc. of the Tenth Marcel Grossman Meeting on General Relativity, Rio de Janeiro, 2003, edited by M. Novello et al. (World Scientific, Singapore, 2006), p. 39.

[31] T. Rindler-Daller and P. R. Shapiro, Mon. Not. R. Astron. Soc. 422, 135 (2012).

[32] J. K. Perring and T. H. R. Skyrme, Nucl. Phys. 31, 550 (1962).

[33] Erick J. Weinberg, Classical Solutions in Quantum Field Theory, Solitons and Instantons in High Energy Physics, Cambridge Monographs in Mathematical Physics (Cambridge University Press, Cambridge, England, 2012).

[34] Ryogo Hirota, J. Phys. Soc. of Japan 33, 1459 (1972).

[35] D. Clowe, M. Markevitch, M. Bradac, A. H. Gonzalez, S. M. Chung, R. Massey, and D. Zaritsky, Astrophys. J. 758, 128 (2012).

[36] J. Lee and M. Baldi, Astrophys. J. 747, 45 (2012).

[37] W. A. Dawson et al., Astrophys. J. Lett. 747 L42 (2012).

[38] M. J. Jee, H. C. Ford et al., Astrophys. J. 661, 728 (2007).

[39] E. W. Mielke and J. A. Vélez Pérez, Phys. Rev. D 75, 043504 (2007).

[40] E. W. Mielke, Phys. Rev. D 18, 4525 (1978).

[41] D.-I. Choi, Phys. Rev. A 66, 063609 (2002); D. Choi, C. W. Lai, M. W. Choptuik, E. W. Hirschmann, S. L. Liebling, and F. Pretorius, Dynamics of axisymmetric (head-on) miniboson star collisions (unpublished).

[42] F. V. Kusmartsev, E. W. Mielke, and F. E. Schunck, Phys. Rev. D 43, 3895 (1991).

[43] F. E. Schunck and E. W. Mielke, Classical Quantum Gravity 20, R301 (2003).

[44] R. Sexl and H. Urbandtke, Relativity, Groups, Particles (Springer Wien, New York, 2001), 4th ed..

[45] J. T. Giblin, Jr., L. Hui, E. A. Lim, and I-S. Yang, Phys. Rev. D 82, 045019 (2010). 